San Jose State University

SJSU ScholarWorks

Master's Theses

Master's Theses and Graduate Research

1989

\title{
A Comparative analysis of the central business districts of Hollister, Watsonville, Santa Cruz, and Los Gatos : 1960-1988
}

Sherry Anne Boboricken

San Jose State University

Follow this and additional works at: https://scholarworks.sjsu.edu/etd_theses

\section{Recommended Citation}

Boboricken, Sherry Anne, "A Comparative analysis of the central business districts of Hollister, Watsonville, Santa Cruz, and Los Gatos : 1960-1988" (1989). Master's Theses. 3125.

DOI: https://doi.org/10.31979/etd.hyed-2vwf

https://scholarworks.sjsu.edu/etd_theses/3125

This Thesis is brought to you for free and open access by the Master's Theses and Graduate Research at SJSU ScholarWorks. It has been accepted for inclusion in Master's Theses by an authorized administrator of SJSU ScholarWorks. For more information, please contact scholarworks@sjsu.edu. 


\section{INFORMATION TO USERS}

The most advanced technology has been used to photograph and reproduce this manuscript from the microfilm master. UMI films the text directly from the original or copy submitted. Thus, some thesis and dissertation copies are in typewriter face, while others may be from any type of computer printer.

The quality of this reproduction is dependent upon the quality of the copy submitted. Broken or indistinct print, colored or poor quality illustrations and photographs, print bleedthrough, substandard margins, and improper alignment can adversely affect reproduction.

In the unlikely event that the author did not send UMI a complete manuscript and there are missing pages, these will be noted. Also, if unauthorized copyright material had to be removed, a note will indicate the deletion.

Oversize materials (e.g., maps, drawings, charts) are reproduced by sectioning the original, beginning at the upper left-hand corner and continuing from left to right in equal sections with small overlaps. Each original is also photographed in one exposure and is included in reduced form at the back of the book. These are also available as one exposure on a standard $35 \mathrm{~mm}$ slide or as a $17^{\prime \prime} \times 23^{\prime \prime}$ black and white photographic print for an additional charge.

Photographs included in the original manuscript have been reproduced xerographically in this copy. Higher quality $6^{\prime \prime} \times 9^{\prime \prime}$ black and white photographic prints are available for any photographs or illustrations appearing in this copy for an additional charge. Contact UMI directly to order.

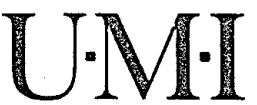



Order Number 1938678

A comparative analysis of the central business districts of Hollister, Watsonville, Santa Cruz, and Los Gatos: 1960 and 1988

Boboricken, Sherry Anne, M.A.

San Jose State University, 1989

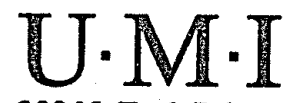

300 N. Zeeb Rd.

Ann Arbor, MI 48106 



\title{
A COMPARATIVE ANALYSIS OF THE CENTRAL BUSINESS DISTRICTS OF
}

HOLLISTER, WATSONVILLE, SANTA CRUZ, AND LOS GATOS: 1960 AND 1988

\author{
A Thesis \\ Presented to \\ The Faculty of the Department of Geography \\ San Jose State University \\ In Partial Fulfillment \\ of the Requirements for the Degree \\ Master of Arts
}

By

Sherry Anne Boboricken

August, 1989 
APPROVED FOR THE DEPARTMENT OF GEOGRAPHY
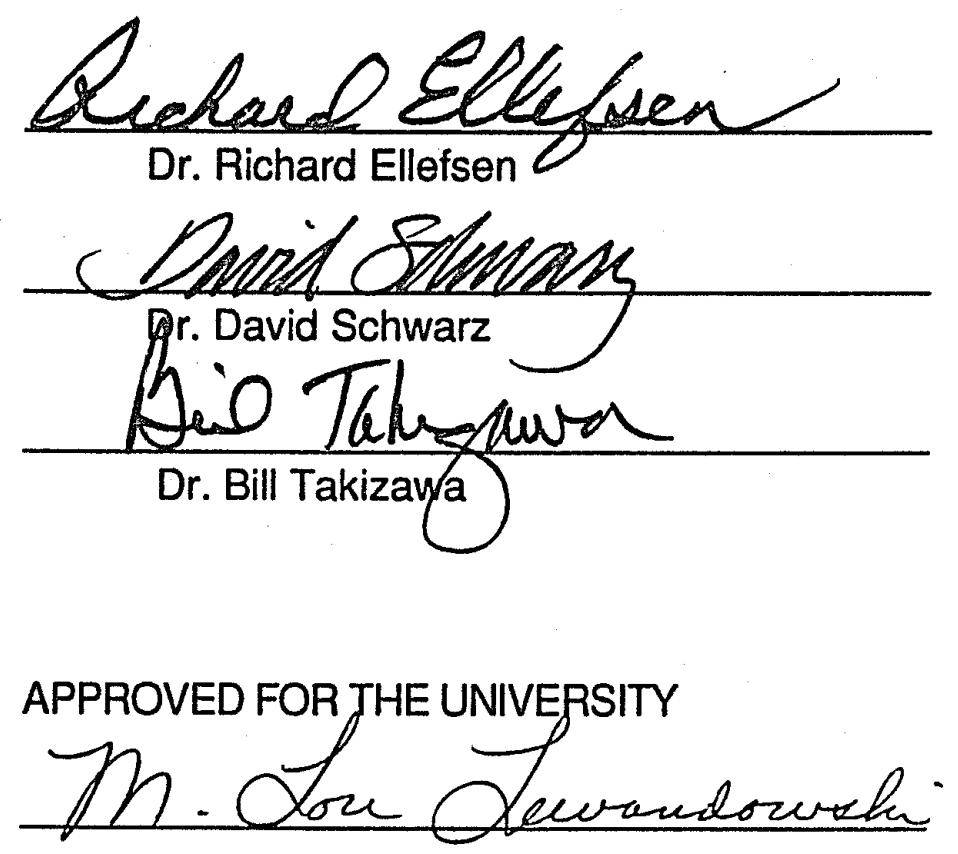


\begin{abstract}
A COMPARATIVE ANALYSIS OF THE CENTRAL BUSINESS DISTRICTS OF HOLLISTER, WATSONVILLE, SANTA CRUZ, AND LOS GATOS: 1960 AND 1988

by Sherry Anne Boboricken
\end{abstract}

This study develops a method for analyzing the evolution of the retail area of central business districts in medium sized cities, and applies it for Los Gatos, Santa Cruz, Watsonville, and Hollister. These cities have evolved or are in the process of evolving from truly independent cities or market towns with small, well defined hinterlands, to cities that are functionally part of the built up metropolitan San Francisco Bay Area.

By analyzing the retail use of floorspace, the place of each city in the trend in retail development was shown. When all of the cities had independent markets, a large percentage of the total retail floorspace was utilized by convenience stores, and a small percentage was utilized by limited appeal stores. Los Gatos and Santa Cruz, being relatively closer to the large metropolitan area in 1988, showed a large increase in the percentage of limited appeal space and a significant decrease in the percentage of convenience floorspace. 


\section{TABLE OF CONTENTS}

page

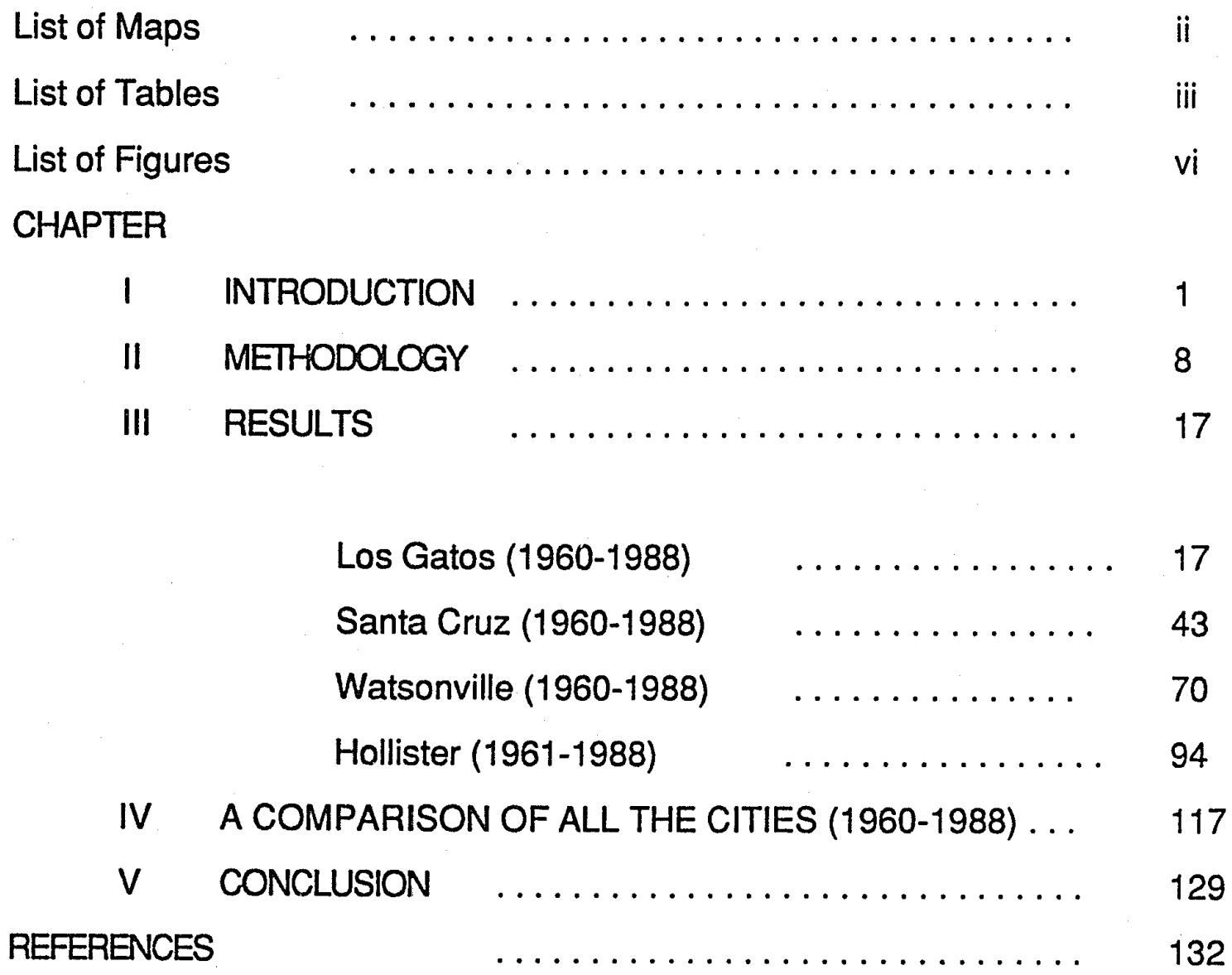




\section{LIST OF MAPS}

MAP

1. Convenience stores in Los Gatos $\mathbf{1 9 6 0}$

2. Convenience stores in Los Gatos 1988

........ 19

3. Limited appeal stores in Los Gatos 1960

$\ldots \ldots \ldots 23$

4. Limited appeal stores in Los Gatos 1988

$\ldots \ldots \ldots 28$

5. Mass appeal stores in Los Gatos $\mathbf{1 9 6 0}$

$\ldots \ldots \ldots \quad 30$

6. Mass appeal stores in Los Gatos 1988

$\ldots \ldots \ldots \quad 34$

7. Convenience stores in Santa Cruz 1960

$\ldots \ldots \ldots \quad 37$

$\ldots \ldots \ldots \quad 44$

8. Convenience stores in Santa Cruz 1988

$\ldots \ldots \ldots \quad 49$

9. Limited appeal stores in Santa Cruz 1960

$\ldots \ldots \ldots \quad 54$

10. Limited appeal stores in Santa Cruz 1988

$\ldots \ldots \ldots \quad 56$

11. Mass appeal stores in Santa Cruz 1960

$\ldots \ldots \ldots \quad 58$

12. Mass appeal stores in Santa Cruz 1988

....... 64

13. Convenience stores in Watsonville 1960

$\ldots \ldots \ldots 71$

14. Convenience stores in Watsonville 1988

$\ldots \ldots \ldots 79$

15. Limited appeal stores in Watsonville 1960 80

16. Limited appeal stores in Watsonville 1988

$\ldots \ldots \ldots$

17. Mass appeal stores in Watsonville 1960

$\ldots \ldots \ldots 83$

18. Mass appeal stores in Watsonville 1988

$\ldots \ldots \ldots \quad 85$

$\ldots \ldots \ldots 87$

19. Convenience stores in Hollister 1961

$\ldots \ldots \ldots 95$

20. Convenience stores in Hollister 1988

$\ldots \ldots \ldots 98$

21. Limited appeal stores in Hollister 1961

$\ldots \ldots \ldots \quad 103$

22. Limited appeal stores in Hollister 1988

$\ldots \ldots \ldots 106$

23. Mass appeal stores in Hollister 1961

$\ldots \ldots \ldots \quad 107$

24. Mass appeal stores in Hollister 1988

$\ldots \ldots \ldots 108$ 
1. Floorspace given to 1960 stores not on the aerial photograph

2. Demographic for all cities

3. Convenience stores in Los Gatos $\mathbf{1 9 6 0}$ by Standard Land Use Code

4. Statistics for Los Gatos's CBD:

1960,1988 , changes 1960-1988

5. Statistics for Los Gatos's "main street": 1960, 1988, changes 1960-1988

6. Convenience stores in Los Gatos 1988

by Standard Land Use Code

7. Limited Appeal stores in Los Gatos $\mathbf{1 9 6 0}$ by Standard Land Use Code

8. Limited Appeal stores in Los Gatos 1988 by Standard Land Use Code

9. Mass Appeal stores in Los Gatos $\mathbf{1 9 6 0}$ by Standard Land Use Code

10. Mass Appeal stores in Los Gatos 1988 by Standard Land Use Code

11. Statistics for Santa Cruz's CBD: 1960, 1988, changes 1960-1988 
TABLE

\section{LIST OF TABLES}

12. Statistics for Santa Cruz's "main street":

page 1960, 1988, changes 1960-1988

13. Convenience stores in Santa Cruz 1960 by Standard Land Use Code

14. Convenience stores in Santa Cruz 1988 by Standard Land Use Code

15. Limited Appeal stores in Santa Cruz 1960 by Standard Land Use Code

16. Limited Appeal stores in Santa Cruz 1988 by Standard Land Use Code

17. Mass Appeal stores in Santa Cruz 1960 by Standard Land Use Code

18. Mass Appeal stores in Santa Cruz 1988 by Standard Land Use Code

19. Statistics for Watsonville's CBD: 1960,1988 , changes 1960-1988

20. Statistics for Watsonville's "main street": 1960, 1988, changes 1960-1988

21. Convenience stores in Watsonville 1960 by Standard Land Use Code

22. Convenience stores in Watsonville 1988 by Standard Land Use Code 
TABLE

LIST OF TABLES

23. Limited Appeal stores in Watsonville 1960

page

by Standard Land Use Code

24. Limited Appeal stores in Watsonville 1988

84

by Standard Land Use Code

25. Mass Appeal stores in Watsonville $\mathbf{1 9 6 0}$

88

by Standard Land Use Code

26. Mass Appeal stores in Watsonville 1988

by Standard Land Use Code

27. Convenience stores in Hollister 1961

by Standard Land Use Code

28. Convenience stores in Hollister 1988

by Standard Land Ụse Code

29. Limited Appeal stores in Hollister 1961

104

by Standard Land Use Code

30. Limited Appeal stores in Hollister 1988

by Standard Land Use Code

31. Mass Appeal stores in Hollister 1961

110

by Standard Land Use Code

32. Mass Appeal stores in Hollister 1988

112

by Standard Land Use Code

33. Statistics for Hollister's CBD:

1960,1988 , changes 1960-1988

34. Statistics for Hollister's "main street": 1960, 1988, changes 1960-1988 


\section{LIST OF FIGURES}

FGURE

page

1. Percentage of floorspace by type for Los Gatos 24

Pie diagrams of:

CBD 1960, the main street only 1960

CBD 1988, the main street only 1988

2. Percentage of floorspace by type for Santa Cruz

Pie diagrams of:

CBD 1960, the main street only 1960

CBD 1988, the main street only 1988

3. Percentage of floorspace by type for Watsonville

78

Pie diagrams of:

CBD 1960, the main street only 1960

CBD 1988, the main street only 1988

4. Percentage of floorspace by type for Hollister

97

Pie diagrams of:

CBD 1961, the main street only 1961

CBD 1988, the main street only 1988

5. Percentage of floorspace by type for 1960 (1961).......

Pie diagrams of the CBDs in:

Los Gatos 1960, Santa Cruz 1960

Watsonville 1960, Hollister 1961

6. Percentage of floorspace by type for main streets $1960 \ldots$.

Pie diagrams of the "main streets" in:

Los Gatos 1960, Santa Cruz 1960

Watsonville 1960, Hollister 1961

7. Percentage of floorspace by type for 1988

Pie diagrams of the CBDs in:

Los Gatos 1960, Santa Cruz 1960

Watsonville 1960, Hollister 1961

8. Percentage of floorspace by type for main streets $1988 \ldots \ldots$

Pie diagrams of the "main streets" in:

Los Gatos 1960, Santa Cruz 1960

Watsonville 1960, Hollister 1961 


\section{CHAPTER 1}

\section{INTRODUCTION}

The retail goods and services in all towns, large and small, are readily separable into three general categories: mass appeal stores, limited appeal stores, and convenience stores. "With the Industrial Revolution, masses of people were forced into a money economy, but held at an extremely low level of ability to consume. Thus, the earliest of retailing establishments were rather general in nature, having to depend upon the selling of mass appeal goods." (Vance 1977, 410) Although mass appeal stores are no longer the exclusive type of retail establishment, they still use the majority of retail space. "As populations increased and disposable income improved, narrow appeal (limited appeal) goods could be introduced to trade." (Vance 1977, 410) As the population increased, convenience stores also appeared, supplying goods and services to "the immediate neighbors, who normally walk to (them) for purchases." (Hartshorn 1980, 345)

The total retail space in all towns is made up of varying percentages of each type. The mix among the three types of retail establishment is a reflection of the anticipated demand for different goods and services which in turn is a result of disposable income and the number of potential consumers. Retail centers catering to a large and wealthy clientele will have a different retail structure from those serving a smaller and more common market. It then is possible to extrapolate from the structure of the retail center the socioeconomic 
characteristics of the market area it serves. If the relative proportion of the retail types is the same for a city over time, it is an indication that the population is uniform. If the retail configuration in more than one city is similar, the situation of the city will also be similar.

The type of retail business in the central business districts of cities changes for two different reasons. First, they change because the market itself changes. When a higher percentage of the population has a larger discretionary income, the demand for more limited appeal goods increases. Secondly, the percentages of retail types changes as a result of the change in the relative proximity of the city to a large urban population. As the large metropolitan area expands, peripheral cities become relatively closer to the large population. As a result of this, the size of a small city's hinterland ${ }^{1}$ enlarges to include communities that were formerly more economically independent. This increase in the population of the hinterland allows for goods needing a higher threshold to be profitable. Either type of change will manifest itself in a different mixture of types of retail goods and services.

An analysis of this trend in retail development is useful in predicting the future retail land use of the central business district.

1 A city's hinterland is the aerial extent of the influence of a city upon the surrounding area. The boundary is at the point beyond which a consumer would choose to shop in the next city. 
A comparison of the proportion of convenience space, limited appeal space, and mass appeal space in 1988 to the percentages in 1960 , demonstrates the direction that the cities are developing. Predictions can be made concerning the future of the Central Business District (CBD).

The purpose of this study is to look at the retail configuration of the Central Business Districts of Hollister, Watsonville, Santa Cruz, and Los Gatos for this period. All of the retail establishments in the CBDs of these towns, as in other towns, consist of a mixture of all three of these retail categories. The percentage of each type varies for each city. The percentages also change within each CBD over time, as the city evolves.

In 1960, all of the cities in the study were basically market towns, but now those closest to the metropolitan Bay Area have changed markedly. By analyzing the evolution that has taken place from 1960 to 1988 , one can predict the downtown retail land use in the future as the Bay Area continues to grow. The Bay Area is steadily becoming one large, continuous city. Los Gatos in 1960 was an independent city, but now is adjacent to San Jose and to other west-bay cities. Downtown Santa Cruz is the center of a node connected to the San Francisco Bay Area by Highway 17. Hollister and Watsonville, however, are still truly independent cities with distinct hinterlands. As the contiguously built up area continues to expand, it is foreseeable that they too will become part this contiguous urban zone. Disposable income has also increased in all of the cities throughout the greater San Francisco Bay Area.

The comparison (between 1960 and 1988) of the retail 
structures of the four cities show both spatial and temporal continua. Los Gatos, closest to the metropolitan area of San Francisco, has been absorbed and integrated into it and displays the most marked change in its retail structure. Santa Cruz, further away, but linked to the metropolitan area by a commuter highway, also has had significant changes in its retail make-up. Although not as dramatic as that in Los Gatos, the trend stemming from stronger links to the metropolitan area is clear. Hollister and Watsonville, far from the core of the metropolitan area, remain largely unaffected by it and still serve local market areas. Their retail compositions have changed the least since 1960.

The purpose of this study is to develop a method for analyzing the evolution of the retail area of central business districts in medium sized cities, and to apply it for Hollister, Watsonville, Santa Cruz, and Los Gatos, which have evolved or are evolving from truly independent cities or market towns with small, well defined hinterlands, to cities that are functionally part of the built up metropolitan Bay Area. As the geographer Harold Carter has stated: "The town as a unit feature of the Earth's surface has, like all other features, two associated aspects. The first is location or position, the second is form and internal structure." (Carter 1971, 7) Following this dictate, this study will look at the internal structure of the downtown and relate it to the relative location of the city. As the relative position of the city changes, so will its internal structure and land use. The techniques used in this spatial and quantitative analysis of urban retail land use should be universally applicable to the study of CBDs of other medium sized cities. 
Both the increase in disposable income, and the increase in proximity to other cities should, theoretically, cause an increase in the percentage of limited appeal space. In Santa Cruz, and especially in Los Gatos, one would expect a much greater proportion of limited appeal stores which would need to draw upon the patronage of the surrounding cities in order to be profitable. Hollister and Watsonville should be able to support a greater percentage of limited appeal goods in the central business districts than they did in 1960 mostly because of an increase in affluence but partially because of propinquity to other cities.

The percentage of floorspace used by convenience stores should decrease in all of the CBDs from 1960 to 1988 , as the pressure from increased rents forces them out of the CBD. The convenience stores that remain in the CBD should be moving towards the periphery of the retail area. The ratio of convenience space to limited appeal space is one of the best indicators of the position of the city's CBD in the trend of retail evolution.

Establishments selling mass appeal goods always dominate in the central business districts of medium sized cities. Further, the percentage of mass appeal retail space tends to remain fairly constant or increase slightly over time. As the CBD retail area evolves, the type of mass appeal store varies. The stores get smaller and become more specialized. The large department stores, which were once a major feature on the "main street," move out, frequently to malls. Even if these large department stores are replaced by other mass appeal stores, the stores tend to carry more specialized merchandise. As the downtowns 
develop, a change in shopping periodicity would also be expected. The downtowns evolve into places where people come to shop on the weekends. This is turn produces a significant increase in the percentage of restaurants and cafes.

The percentage of limited appeal space also increases as part of this process. An increase in both discretionary income and the proximity of a city to a larger urban core allows for a greater percentage of limited appeal space within the CBD. As the stores become smaller and more specialized, they evolve into limited appeal stores. These limited appeal stores attract the weekend shoppers who come to purchase items which are not part of their everyday needs.

Convenience stores are a very interesting feature of the central business district. They have changed both in character and importance. In the past, they were frequently owned by a single family which lived adjacent to the store. Now, chain stores like 7-11 are replacing them. Epitomized by the "corner grocery store," they cater largely to the surrounding neighborhood. As the downtown evolves, they are among the first stores to be pushed out of the CBD. Although the amount of space they use has decreased, they still hang on to a considerably larger percentage of corner locations than their floorspace would indicate.

A comparison of the retail on the "main street" with the retail in the CBD as a whole is very useful. One would expect a higher percentage of mass appeal space on the main street because clustering proves advantageous to stores which sell mass appeal goods. They also tend to generate a more predictable and steady revenue and, therefore, 
they can afford to pay the higher rents. Limited appeal and convenience stores command only a certain market regardless of location and, therefore, do not make significantly more money if they are located on the "main street".

This method of analyzing the CBDs of Hollister, Watsonville, Santa Cruz, and Los Gatos, applies a universal and comprehensive way of analyzing a CBD of a medium sized city. Any method of analyzing the retail area of the CBD must necessarily use a combination of spatial and quantitative analysis. The method explained in the following chapter utilized both statistical analysis and spatial mapping. This method determines where each city falls in the trend of development on the periphery of a large urbanizing area. Determining where these four cities fall in the trend, permits a forecast to be made about their future development and land use patterns. All of the cities are expected to continue to evolve in the direction they are already moving. 


\section{CHAPTER II}

\section{METHODOLOGY}

\section{Choosing the Cities and Time Periods}

Four cities were chosen for the study: Los Gatos, Santa Cruz, Watsonville, and Hollister. All of these cities have approximately the same size central business districts; however, their nearness to the large urban core of the San Francisco Bay Area differs. Los Gatos is no longer an independent market city. It has become part of the contiguous built-up metropolitan Bay Area. With the outward expansion of the built-up area, Santa Cruz has become relatively closer to the urban core. It is now a retail node linked by the highway. Watsonville and Hollister, however, still have fairly well defined hinterlands. Their downtowns are patronized almost exclusively by local residents and the surrounding, largely rural population. Each city's location relative to the major urban core places it at a different, sequential point in the trend of retail development its CBD.

1960 was chosen as the base year because at that time all of these cities were independent market cities serving their immediate hinterlands. By 1960, the size of each CBD was also about the same size as it is now. The "main streets" were already built up. This is important because one sees the change withir the CBD rather than the expansion of it. 


\section{Delineation of the Study Area - Retail}

Since the objective of this study is to analyze and compare the retail space in the central business districts of the cities, all non-retail establishments were eliminated. Retail establishments include retail sales, retail services, and vacant retail stores. Offices that are not strictly retail establishments, such as lawyer's, doctor's, CPA's, and government offices, are not included. If optometry stores mostly sell glasses, they are considered a limited appeal retail store. For 1960, the name of the store is used to judge the function. In order to keep the areas studied for each city and time period comparable, no shopping centers or retail establishments above street level were included, since very few retail establishments exist on the second stories.

The Polk Directory for each city was used to determine the name and type of establishment for 1960 for each address (1961 was used for Hollister because no Polk directory was made for 1960). Vacancies that fell within the contiguous retail zone were counted as retail vacancies. The Polk directories allow for the re-creation of the retail area in the past. For each address, the Polk directory lists the name of the retail establishment and a brief description of its merchandise. The directory lists all of the addresses used at the time. By combining the knowledge obtained from the Polk directories with the floorspace obtained from direct field observation in 1988, this study determines the percentage of space used by each type of retail establishment. 


\section{Name, Address, Type of Establishment, and Floorspace}

Three measuring options were considered for determining the importance of each establishment in this study: 1. the number of each type of establishment, 2. the length of street frontage, and 3. the floorspace area. Floorspace was chosen, because the number of establishments as well as the frontage length can be deceptive. The number of stores would exaggerate the importance of small stores and diminish the importance of large stores such as department stores. Rents are also determined by floorspace rather than by frontage space, making their profitability dependent on floorspace.

To determine the floorspace, aerial photographs were used. The aerial photographs were obtained from the Public Works Department or from the Engineering Department of each city. The aerial photograph for Hollister was at a scale of $1 "=200$, Watsonville and Santa Cruz were at $1^{\prime \prime}=100^{\prime}$, and the only one available for Los Gatos was at $1^{\prime \prime}=50^{\prime}$. At all of these scales the buildings were clearly seen and of a measurable size. The various scales did not affect the accuracy of the results. For the purposes of this study, $1 "=50$ ' was a little cumbersome because of the large number of sheets needed to cover the downtown area.

For 1988, the name, address, and type of establishment was obtained from direct field observation. After obtaining the aerial photographs, all of the stores and buildings were identified. A rough field map was made of each block, as well as writing the addresses directly onto the aerial photograph. The address, the name of the store, the type of store, and the estimated percent of building occupied were 
noted on the field map.

The dimensions of each building were measured on the aerial photograph, multiplied to find the area, and calculated to scale. One half meter was subtracted to account for wall width. Direct field observation was used to negate the amount of area due to roof overhang. The length of the overhang was paced off on the ground. Buildings that are used by more than one store were proportioned by estimating the percent of the frontage that they take up by pacing off the longer distances and using a tape to measure the shorter distances. The floorspace determined by measuring the buildings on the aerial photograph are used for addresses that appear both in 1960 and 1988. Buildings in 1960 (1961) that were not on the aerial photograph, were given values approximating the size of that type of store. The retail area for stores in 1960 that could not be determined by the 1988 data had to be given some value. If the unmeasureable stores were all given the same value for floorspace, it would greatiy exaggerate the floorspace of traditionally small stores such as gas stations and cigar shops.

If there were only a few stores of a specific type, the store with unknown floorspace was given an average of all stores: $250 \mathrm{~m}^{2}$. If an address that existed in 1960 was eliminated by 1988 , because a larger store had moved in that used more than one old store space, the 1988 floorspace was divided up evenly among the new addresses. If two or more different addresses exist in 1988 for one in the past, then the sum of the floorspace for all of the new addresses is used for the store in 1960. Buildings for 1988 that were not on the aerial photograph were 
measured on the ground. Buildings that were obviously constructed or reconstructed between 1960 and 1988, even if they had an address that existed in 1960 and 1988, were given average values for 1960 .

TABLE 1: $\quad$ Floorspace given to 1960 stores not on the aerial photograph

\begin{tabular}{|c|c|c|}
\hline type of store & floorspace $m 2$ & standard land use value \\
\hline beauty/barber & 71 & 623 \\
\hline gas station & 76 & 553 \\
\hline cigar shops & 84 & 599 \\
\hline real estate & 92 & 615 \\
\hline laundry/dry cl & 96 & 621 \\
\hline florist & 118 & 599 \\
\hline men's clothes & 136 & 561 \\
\hline travel agents & 138 & 492 \\
\hline shoe repair & 144 & 625 \\
\hline women's clothes & 159 & 562 \\
\hline body shop & 163 & 641 \\
\hline cafe & 163 & 581 \\
\hline bakery & 165 & 546 \\
\hline antiques & 169 & 593 \\
\hline bars & 170 & 582 \\
\hline restaurant & 194 & 581 \\
\hline furs & 200 & 568 \\
\hline appliances & 200 & 572 \\
\hline shoe stores & 209 & 566 \\
\hline church goods & 216 & 599 \\
\hline pharmacy & 222 & 591 \\
\hline corner market & 232 & 545 \\
\hline clothes & 260 & 565 \\
\hline lumber & 284 & 521 \\
\hline liquor stores & 289 & 592 \\
\hline auto pts/tires & 374 & 552 \\
\hline printers & 374 & 633 \\
\hline furniture & 549 & 571 \\
\hline thrift stores & 581 & 593 \\
\hline car dealers & 600 & 551 \\
\hline all others & 250 & \\
\hline
\end{tabular}




\section{Classifications}

Each store is classified in two ways. The first classification divides into three general categories: convenience, limited appeal, and mass appeal. Convenience stores are shops that are patronized mainly because of their location. "An isolated grocery or drugstore or tavern typifies this class." (Hartshorn 1980, 345) Convenience stores in general have a very low threshold and sell "low order" goods. They tend to have names which indicate their type, prices which are not competitive, and few or no promotional sales. This category includes the types of stores listed below.

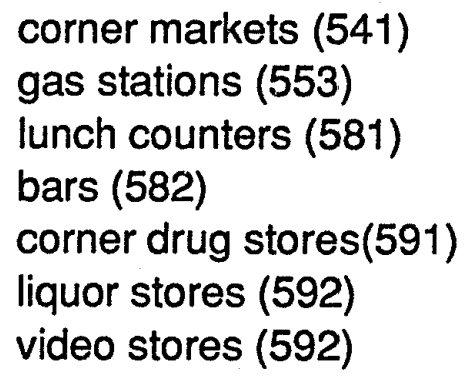

mini florists (599)

hour photo (599)

dry cleaners/laundry (621)

beauty salons/barber shops (623)

shoe repair shops (625)

photo copy stores (633)

watch repair (649)

Each store was also given its three digit classification from the Standard Land Use Coding Manual. These are the numbers listed above in parenthesis. Because video rental stores did not have a land-use code, they were given the same number as liquor stores (592) because of their ultra-convenience type.

Limited appeal stores sell merchandise or services which not everyone buys, either because of great expense or because of lack of 
specific interest. This category includes pet stores, hobby shops, antique stores, specialty auto dealers, glass stores, etc. These stores have a high threshold and low frequency. (Hartshorn 1980, 344) Limited appeal stores are more diverse than convenience stores, but like convenience stores, they rarely have sales and do not necessarily have competitive prices. Mass appeal stores are shops which sell goods frequently purchased by most people. Stores selling such things as women's clothing are included in mass appeal even though not everyone buys women's clothes. Although these stores are sub-classifications, they are still part of the mass appeal classification of clothing. Mass appeal includes all or most of the types of stores listed in the following list.

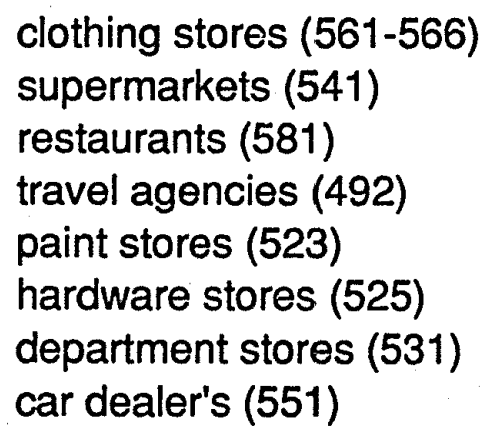

appliance stores (572) books, records etc. (594) banks (611) car repair (641) jewelers (597) florists (599) theaters (721) real estate agencies (615)

Specialty shops within these categories such as spiritual book stores, luxury auto dealerships, stores which sell uniforms, and specialty restaurants are classified as limited appeal stores. The above list does not cover all of the types of mass appeal store. If a grocery store in 1960 was small, it was considered a convenience store. If it was large, it was considered a mass appeal store. In 1988, large chain grocery stores, such as Safeway, were considered mass appeal stores. All others 
were considered convenience stores.

\section{Sarting the Data}

Once the floorspace and type were determined for each store, they were sorted three different ways. The most useful for study was sorting by type: $C$ for convenience, $L$ for limited appeal, or $M$ for mass appeal. Within each type, the stores were sorted by their Standard Land Use Manual classification. This sorting allows for the calculation of the total area and percent of sub-classifications within the type, such as banks, restaurants, and gas stations. Sub-ciassifications are useful for analyzing retail land use within the general type $(C, M, L)$. The stores were also sorted by block for the purpose of spatial analysis and generating maps.

From the above sorted data, statistics were generated for each city's entire central business district and for the "main street". The "main street" is defined as the street with the greatest amount of retail floorspace. These streets are easily identified in all of the cities, frequently containing more that half of the total retail floorspace in the city. Calculations were made for each time period, of the number of store of each type, the total square meters of the type, the average floorspace of the type, the standard deviation of the average, and the relative proportion of each type. Values have also been calculated for the total retail area. These statistics allow for a comparison from 1960 to 1988 , as well as a comparison of the "main street" to the CBD as a whole. A list of changes from 1960 to 1988 was also made for each downtown 
and for the "main street".

\section{Maps of the Retail Types for Each City}

Illustrating the retail distribution, spatial maps were generated for each city and time period. The base maps for all of the cities were made from the aerial photograph at a scale of 1:6000 $\left(1 "=500^{\prime}\right)$. The street widths include the sidewalk space on both sides, because sidewalks are not part of the retail area. The area for each store is a percent of the total retail space for the block that it constitutes. Spaces have been left blank for non-retail land use, including parking lots. For each city, six maps were made: one for each year and for each classification--convenience, limited appeal, and mass appeal.

\section{Pie Graphs}

In order to analyze the CBDs quantitatively, six sets containing four pie graphs each have been generated from the statistics: one for each city, one for 1960 (1961), and one for 1988. Each pie graph is a circle divided by percent of convenience stores, limited appeal, mass appeal, and vacant retail space. The set for each city includes: one for the downtown in 1960, one for the "main street" in 1960, one for the downtown in 1988, and one for the "main street" in 1988. 
CHAPTER III

AN ANALYSIS OF EACH CITY 1960-1988

\section{LOS GRTOS}

\section{0-1988}

Los Gatos is close to a number of cities, including San Jose. Its close proximity to the large population base of the Bay Area gives it a different character from the other cities in the study. Los Gatos' central business district consists of North Santa Cruz Avenue, from W. Main Street to Saratoga Avenue. Perpendicular to N. Santa Cruz Avenue the CBD extends for only one block to the east and to the west. The CBD also includes East and West Main Street from Pageant to N. Santa Cruz Avenue. (see map 1) A large part of the retail outside of the CBD in Los Gatos is located just north of the CBD and is not considered part of the CBD because of the mall configuration with off-street parking in front of the stores.

In 1960, the city reported 9,040 residents, making it larger than Hollister but smaller than either Santa Cruz or Watsonville. The downtown consisted of $26,22.8$ square meters of retail floorspace. Eighty-three percent of the retail stores in 1960 were located within the CBD. (see table 2) By 1988, the amount of retail floorspace in the CBD grew by 22,151 square meters, or by over $84 \%$. Most of this growth was off the "main street". The population grew to 27,950, a change of over $200 \%$ over 1960 . Other than the downtown retail area, Los Gatos now has 
TABLE: 2 DEMOGRAPHICS FOR THE CITIES

\begin{tabular}{|c|c|c|c|c|}
\hline & HOUSTER & WATSONVILLE & SANTACRUZ & LOSGATOS \\
\hline POPULATION $1960_{1}$ & 6070 & 13290 & 25600 & 9040 \\
\hline POPULATION $1987_{2}$ & 16800 & 28550 & 46900 & 27950 \\
\hline $\begin{array}{l}\text { CHANGEINPOP } \\
\% \text { CHANGE IN POP }\end{array}$ & $\begin{array}{l}10730 \\
177\end{array}$ & $\begin{array}{l}15260 \\
115\end{array}$ & $\begin{array}{l}21300 \\
83\end{array}$ & $\begin{array}{l}18910 \\
209\end{array}$ \\
\hline $\begin{array}{c}\text { PER CAPITA INCOME } \\
1983\end{array}$ & 7034 & 8212 & 9808 & 17254 \\
\hline $\begin{array}{l}\text { RETAIL STORES } 1960 \\
\text { TAXABLE TRANS } \$ 1960 \\
\text { \# OF RETAIL IN CBD } \\
\% \text { OF RETAIL IN CBD }\end{array}$ & $\begin{array}{l}136 \\
9894 \\
131 \\
96\end{array}$ & $\begin{array}{l}252 \\
22786 \\
189 \\
75\end{array}$ & $\begin{array}{l}468 \\
43457 \\
182 \\
39\end{array}$ & $\begin{array}{l}184 \\
24560 \\
152 \\
83\end{array}$ \\
\hline
\end{tabular}

several small, both mass and convenience type shopping malls. The per capita income in 1983 was $\$ 17,254$, more than twice that of Hollister or Watsonville, and more than $\$ 7,000$ greater than the per capita income in Santa Cruz.

\section{Conuenience}

Like the other cities in the study, Los Gatos in 1960, had a substantial percentage, $23.3 \%$, of convenience space within the CBD. Convenience stores were distributed throughout the downtown. Only one block had no convenience stores on it. (see map 1) The largest amount of convenience space was used by barber shops and beauty salons: $22.9 \%$.

The next largest users were laundromats and dry cleaners (19.3\%), corner 


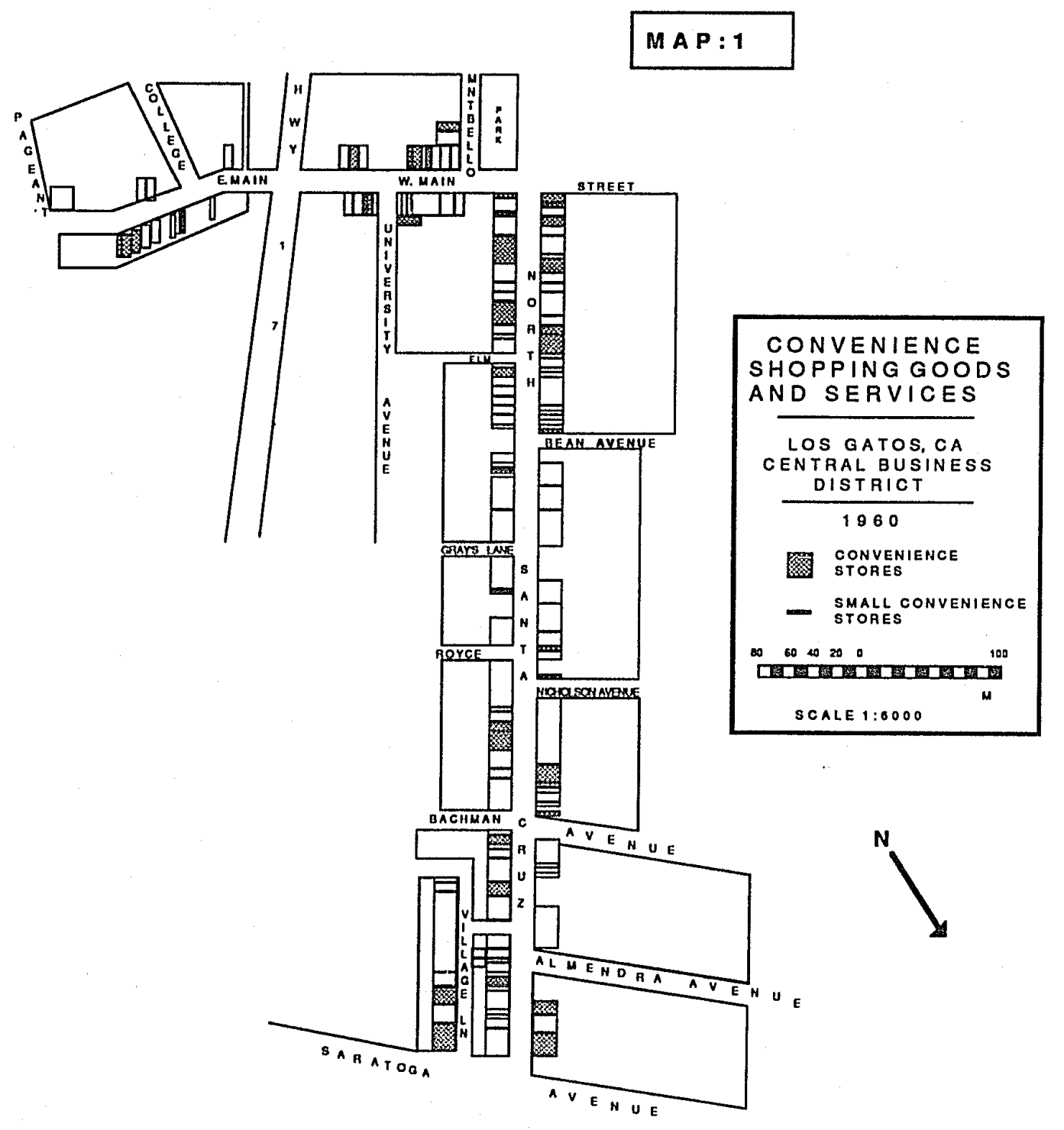


markets $(12.7 \%)$, drug stores (16.4\%), and bars (10.5\%). (see table 3 )

By 1988 , there were several blocks which had no convenience stores on them. (see map 2) There are none on E. Main Street and only a couple on West Main Street. The central blocks of N. Santa Cruz Avenue also have almost no convenience stores on them. The percentage of space occupied by convenience stores decreased by more than half from 1960 to 1988. (see fig. 1; tables 4 and 5) The percent of convenience space on "main street" also decreased by more than $50 \%$. No corner market stores remain in Los Gatos' downtown. Neither are there any gas stations in the CBD, but a few are located nearby on Saratoga Avenue because of the considerable traffic along it. The only two new types of convenience stores are video stores and manicurists. Barber shops and beauty salons increased to $35.2 \%$ of the total convenience space. The percentage of convenience space in laundry/dry cleaners increased to $32.3 \%$. Bars and shoe repair shops still make up a small percentage of the convenience space. (see table 6)

\section{Limited appeal}

Eight point three percent of the total retail space in 1960 consisted of limited appeal stores. As expected, the "main street", N. Santa Cruz Avenue, had a slightly smaller percentage of limited appeal space: $7.7 \%$. The ratio of limited appeal stores to convenience stores was .45; there was about twice as much convenience space as limited appeal space. Although several blocks have no limited appeal stores on them, there is no apparent clustering of these stores. (see map 3) 
TABLE 3: CONVENIENCE STORES IN LOS GATOS IN 1960 BY STANDARD LAND USE CODE

Name of Establishement

Street Address

Standard Type Area $M^{2}$ Land Use

Code

\begin{tabular}{|c|c|c|c|c|c|}
\hline LOS GATOS MARKET & 26 & N. SANTA CRUZ AVE & 541 & $\mathrm{C}$ & 291 \\
\hline S+A FOOD MKT & 17 & N. SANTA CRUZ AVE & 541 & C & 248 \\
\hline CITY HALL MKT GRO & 45 & E. MAIN STREET & 541 & $\mathrm{C}$ & 240 \\
\hline MALATESTA'S FLYING A SERV - gas *** & 40 & N. SANTA CRUZ AVE & 553 & $\mathrm{C}$ & 76 \\
\hline HAPE'S AL CHEVRON SERV - gas *** & 59 & N. SANTA CRUZ AVE & 553 & $\mathrm{C}$ & 76 \\
\hline CRIDER'S PAUL MOBIL - gas *** & 155 & N. SANTA CRUZ AVE & 553 & C & 76 \\
\hline BOB'S RICHFFIELD SERV - gas & 335 & N. SANTA CRUZ AVE & 553 & C & 76 \\
\hline R + H RICHFIELD GAS STATION *** & 27 & W. MAIN STREET & 553 & C & 76 \\
\hline SPEZIALE BROS TEXACO - gas & 233 & N. SANTA CRUZ AVE & 553 & $\mathrm{C}$ & 65 \\
\hline DAVE'S DONUTSHOP & 91 & W. MAIN STREET & 581 & $\mathrm{C}$ & 50 \\
\hline WILDCAT SPORT SHOPIKOZY KAFE & 133 & N. SANTA CRUZ AVE & 582 & C & 306 \\
\hline BLACK WATCH - bar & 142 & N. SANTA CRUZ AVE & 582 & C & 132 \\
\hline CLUB SEVEN TAVERN & 7 & N. SANTA CRUZ AVE & 582 & C & 120 \\
\hline HAPPY DRAGON THE SOCIAL WELFARE & 78 & W. MAIN STREET & 582 & C & 88 \\
\hline LOS GATOS PHARM THE & 47 & E. MAIN STREET & 591 & C & 243 \\
\hline NORTHGATE CUT RATE DRUG STORE **** & 349 & N. SANTA CRUZ AVE & 591 & C & 222 \\
\hline GREEN'S PHARM & 218 & N. SANTA CRUZ AVE & 591 & C & 210 \\
\hline DUNCAN'S PHARM & 29 & N. SANTA CRUZ AVE & 591 & $\mathrm{C}$ & 170 \\
\hline CORNERDRUGSTORE & 1 & N. SANTA CRUZ AVE & 591 & C & 162 \\
\hline GENE'S COUNTRY STORE LIQUORS *** & 217 & N. SANTA CRUZ AVE & 592 & C & 289 \\
\hline SHAMROCK LIQUORS & 49 & E. MAIN STREET & 592 & c & 95 \\
\hline FORD BROS CLNS & 33 & N. SANTA CRUZ AVE & 621 & C & 345 \\
\hline GATITO CLEANERS & 312 & N. SANTA CRUZ AVE & 621 & C & 214 \\
\hline VILLAGE LAUNDERETIE & 368 & VILLAGE LANE & 621 & C & 180 \\
\hline GEM CITY FRENCH LNDRY & 11 & UNIVERSITY AVE & 621 & $\mathrm{C}$ & 123 \\
\hline TIP TOP CLNS & 216 & N. SANTA CRUZ AVE & 621 & C & 118 \\
\hline SELF SERV LAUNDERETTE & 9 & MONTEBELOWAY & 621 & $\mathrm{C}$ & 116 \\
\hline LOS GATOS CLNS & 88 & W. MAIN STREET & 621 & C & 91 \\
\hline LARRY'S SHOE SERVLILLY'S BARBER SHOP & 12 & N. SANTA CRUZ AVE & 623 & C & 245 \\
\hline RUDY'S BARBER SHOP & 29 & E. MAIN STREET & 623 & C & 154 \\
\hline VANITY FAIR BEAUTY SALON & 50 & N. SANTA CRUZ AVE & 623 & $\mathrm{C}$ & 154 \\
\hline REYNOLDS BARBER SHOP & 110 & W. MAIN STREET & 623 & $\mathrm{C}$ & 128 \\
\hline LEMONTE BEAUTY SALON & 65 & W. MAIN STREET & 623 & C & 103 \\
\hline LLOYD'S HAIRCUTTING SHOP *** & 272 & N. SANTA CRUZ AVE & 623 & C & 96 \\
\hline VILLAGE BARBER & 332 & N. SANTA CRUZ AVE & 623 & C & 96 \\
\hline LITTLE VILLAGE BEAUTY SHOPPE & 360 & VILLAGE LANE & 623 & C & 96 \\
\hline DENNY'S TAXIUOE'S BARBER SHOP & 139 & N. SANTA CRUZ AVE & 623 & C & 78 \\
\hline ROBERTA'S TOWN HOUSE BEAUTY SALON & 34 & N. SANTA CRUZ AVE & 623 & $\mathrm{C}$ & 75 \\
\hline ELPATIO BEAUTY SALON & 318 & N. SANTA CRUZ AVE & 623 & C & 71 \\
\hline BOONE'S BARBER SHOP & 114 & N. SANTA CRUZ AVE & 623 & $\mathrm{C}$ & 60 \\
\hline LACANADA BUILDING- barber & 3 & N. SANTA CRUZ AVE & 623 & C & 47 \\
\hline CATANIA JOHN SHOE REPAIR & 23 & W. MAIN STREET & 625 & C & 106 \\
\hline
\end{tabular}


STRINGER'S SHOE REPAIR ***

$D+D$ SHOE SERV

NUMBER OF CONVENIENCE STORES

TOTAL AREA M2 OF CONVENIENCE SPACE

AVE M2 OF C

$\%$ OF C (AREA)

SD FORC
219 N. SANTA CRUZ AVE 625 C 7

120 W. MAIN STREET 625 C 5

44

6135

139

23.3

79 


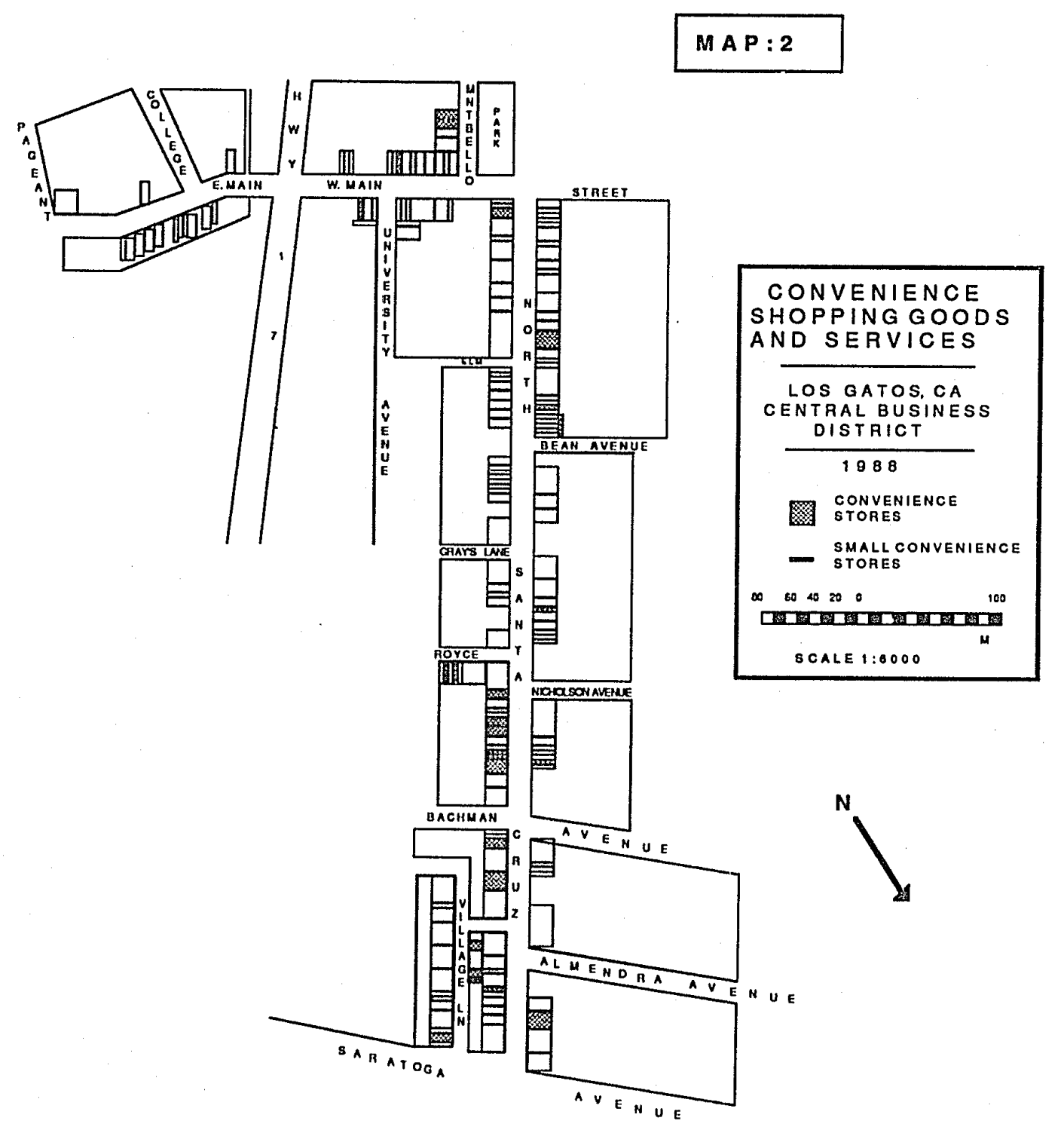


Fig. 1

Percentages of retail land use in the CBD

LOS GATOS

LOSGATOS 1960

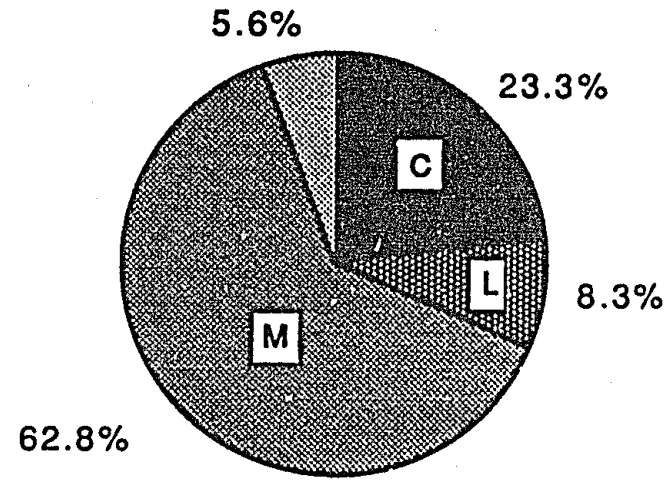

LOS GATOS 1988

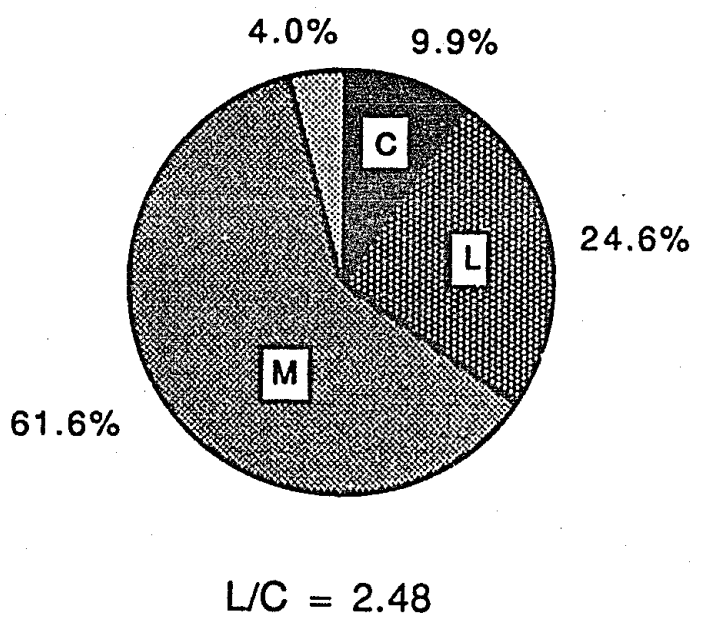

LOS GATOS 1960

THE MAIN STREET ONLY

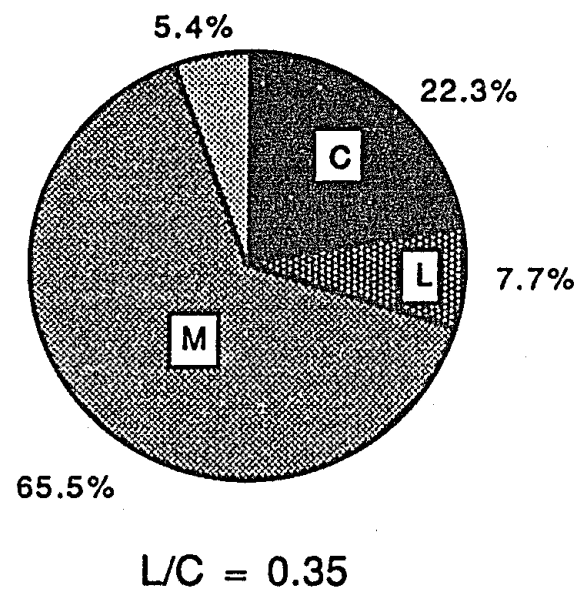

LOS GATOS 1988

THE MAIN STREET ONLY

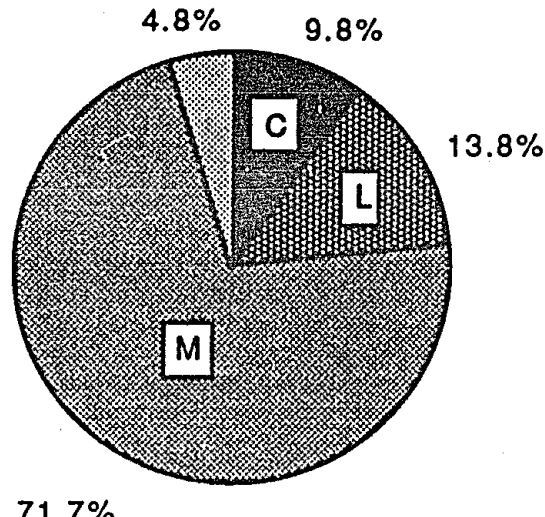

$L / C=1.41$ 
TABLE 4: STATISTICS FOR THE CENTRAL BUSINESS DISTRICT IN LOS GATOS

STATISTICS FOR LOS GATOS IN 1960

\begin{tabular}{|c|c|c|c|c|c|c|c|}
\hline $\begin{array}{l}\text { NUMBER OFC } \\
\text { TOTAL M2 OF C } \\
\text { AVE M2 OF C } \\
\% \text { OF C (AREA) } \\
\text { SD FORC }\end{array}$ & $\begin{array}{l}44 \\
6135 \\
139 \\
23.3 \\
79\end{array}$ & $\begin{array}{l}\text { NUMBER OF L } \\
\text { TOTAL M2 OF L } \\
\text { AVE M2 OF L } \\
\% \text { OF L (AREA) } \\
\text { SD FORL }\end{array}$ & $\begin{array}{l}17 \\
2188 \\
129 \\
8.3 \\
70\end{array}$ & $\begin{array}{l}\text { NUMBER OFM } \\
\text { TOTAL M2 OF M } \\
\text { AVE M2 OF M } \\
\% \text { OF M (AREA) } \\
\text { SD FORM }\end{array}$ & $\begin{array}{l}82 \\
16502 \\
201 \\
62.8 \\
190\end{array}$ & $\begin{array}{l}\text { NUMBER OFV } \\
\text { TOTAL M2 OF V }\end{array}$ & $\begin{array}{l}9 \\
1463\end{array}$ \\
\hline $\begin{array}{l}\text { TOTAL NUMBER } \\
\text { TOTAL M2 } \\
\text { AVE M2 OF ALL } \\
\% \text { OF ALL } \\
\text { SD FOR ALI }\end{array}$ & $\begin{array}{l}152 \\
26288 \\
173 \\
100 \\
156\end{array}$ & & & $\begin{array}{l}\% \text { OF C (AREA) } \\
\% \text { OF L (AREA) } \\
\% \text { OF M (AREA) } \\
\% \text { OF } \vee \text { (AREA) }\end{array}$ & $\begin{array}{l}23.3 \\
8.3 \\
62.8 \\
5.6 \\
\end{array}$ & & \\
\hline
\end{tabular}

STATISTICS FOR LOS GATOS IN 1988

\begin{tabular}{|c|c|c|c|c|c|c|c|}
\hline $\begin{array}{l}\text { NUMBER OFC } \\
\text { TOTAL M2 OF C } \\
\text { AVE M2 OF C } \\
\% \text { OF C (AREA) } \\
\text { SD FORC }\end{array}$ & $\begin{array}{l}27 \\
2941 \\
109 \\
9.9 \\
77\end{array}$ & $\begin{array}{l}\text { NUMBER OF L } \\
\text { TOTAL M2 OF L } \\
\text { AVE M2 OFL } \\
\% \text { OF L (AREA) } \\
\text { SD FORL }\end{array}$ & $\begin{array}{l}52 \\
7307 \\
141 \\
24.6 \\
155\end{array}$ & $\begin{array}{l}\text { NUMBER OFM } \\
\text { TOTAL M2 OF M } \\
\text { AVE M2 OF M } \\
\% \text { OF M (AREA) } \\
\text { SD FORM }\end{array}$ & $\begin{array}{l}100 \\
18317 \\
183 \\
61.6 \\
156\end{array}$ & $\begin{array}{l}\text { NUMBER OFV } \\
\text { TOTAL M2 OF V } \\
\% \text { OF V (AREA) }\end{array}$ & $\begin{array}{l}7 \\
1187\end{array}$ \\
\hline $\begin{array}{l}\text { TOTAL NUMBER } \\
\text { TOTAL M2 } \\
\text { AVE M2 OF ALL } \\
\% \text { OF ALL } \\
\text { SD FOR ALL }\end{array}$ & $\begin{array}{l}186 \\
48439 \\
187 \\
100 \\
256\end{array}$ & & & $\begin{array}{l}\% \text { OF C (AREA) } \\
\% \text { OF L (AREA) } \\
\% \text { OF M (AREA) } \\
\% \text { OF V (AREA) } \\
\% \text { L/\%C }\end{array}$ & $\begin{array}{l}9.9 \\
24.6 \\
61.6 \\
4 \\
\\
2.48\end{array}$ & & \\
\hline
\end{tabular}

CHANGE FROM 1960 TO 1988

\begin{tabular}{|c|c|c|c|c|c|c|c|}
\hline $\begin{array}{l}\mathrm{CH} \mathrm{IN} \mathrm{\#} \mathrm{C} \\
\mathrm{CH} M 2 \text { OF C }\end{array}$ & $\begin{array}{l}-17 \\
-3194\end{array}$ & $\begin{array}{l}\mathrm{CH} I N \# \mathrm{~L} \\
\mathrm{CH} M 2 \text { OF L }\end{array}$ & $\begin{array}{l}35 \\
5119\end{array}$ & $\begin{array}{l}\mathrm{CH} \text { IN \# M } \\
\mathrm{CH} \text { M2 OF M }\end{array}$ & $\begin{array}{l}18 \\
1815\end{array}$ & $\begin{array}{l}\mathrm{CH} \text { IN \# V } \\
\mathrm{CH} M 2 \text { OF V }\end{array}$ & $\begin{array}{l}-2 \\
-276\end{array}$ \\
\hline CH AVE M2 OF C & -30 & CH AVE M2 OF L & 12 & CH AVE M2 OF M & -18 & & \\
\hline $\mathrm{CH} \%$ OF C AREA & -13.4 & $\mathrm{CH} \%$ OF L AREA & 16.3 & $\mathrm{CH} \%$ OF M AREA & -1.2 & CH\% OF V AREA & -1.6 \\
\hline CH TOTAL NBR & 34 & & & $\% \mathrm{CH}$ IN \% C & -57.5 & & \\
\hline CH TOTAL M2 & 22151 & & & $\% \mathrm{CH}$ IN \% L & 196.4 & & \\
\hline CH AVE M2 ALL & 14 & & & $\begin{array}{l}\% \mathrm{CH} I N \% M \\
\% \mathrm{CH} \text { IN \% V }\end{array}$ & $\begin{array}{l}-1.9 \\
-28.6\end{array}$ & & \\
\hline$\%$ CH TOT M2 & 84.3 & & & & & & \\
\hline & & & & $\mathrm{CH}$ L/C & 2.13 & & \\
\hline
\end{tabular}


TABLE 5 : STATISTICS FOR THE CENTRAL BUSINESS DISTRICT IN LOS GATOS -- THE MAIN STREET ONLY

STATISTICS FOR LOS GATOS ON THE MAIN ST IN 1960

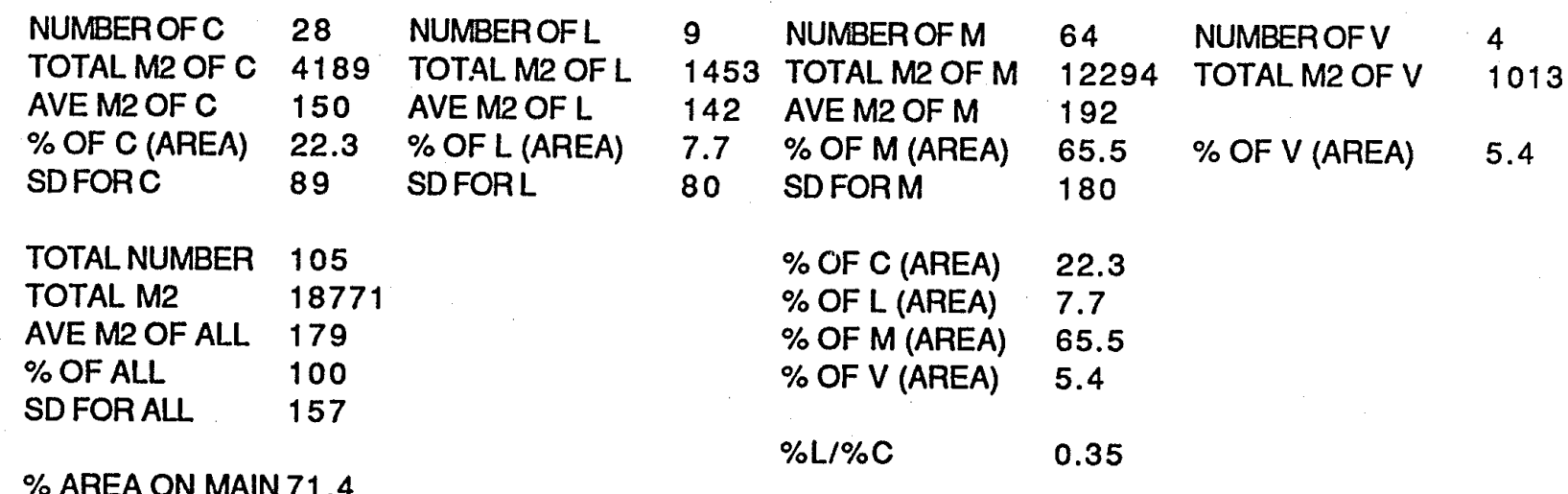

STATISTICS FOR LOS GATOS ON THE MAIN ST IN 1988

\begin{tabular}{|c|c|c|c|c|c|c|c|}
\hline $\begin{array}{l}\text { NUMBER OFC } \\
\text { TOTAL M2 OF C } \\
\text { AVE M2 OF C } \\
\% \text { OF C (AREA) } \\
\text { SDFORC }\end{array}$ & $\begin{array}{l}16 \\
2053 \\
128 \\
9.8 \\
92\end{array}$ & $\begin{array}{l}\text { NUMBER OFL } \\
\text { TOTAL M2 OF L } \\
\text { AVE M2 OF L } \\
\% \text { OF L (AREA) } \\
\text { SD FORL }\end{array}$ & $\begin{array}{l}24 \\
2873 \\
120 \\
13.8 \\
97\end{array}$ & $\begin{array}{l}\text { NUMBER OFM } \\
\text { TOTAL M2 OF M } \\
\text { AVE M2 OF M } \\
\% \text { OF M (AREA) } \\
\text { SDFORM }\end{array}$ & $\begin{array}{l}74 \\
14957 \\
202 \\
71.7 \\
168\end{array}$ & $\begin{array}{l}\text { NUMBEROFV } \\
\text { TOTAL M2 OF V }\end{array}$ & $\begin{array}{l}6 \\
992\end{array}$ \\
\hline $\begin{array}{l}\text { TOTAL NUMBER } \\
\text { TOTAL M2 } \\
\text { AVE M2 OF ALL } \\
\% \text { OF ALL } \\
\text { SD FOR ALL }\end{array}$ & $\begin{array}{l}120 \\
20875 \\
174 \\
100 \\
148\end{array}$ & & & $\begin{array}{l}\% \text { OF C (AREA) } \\
\% \text { OF L (AREA) } \\
\% \text { OF M (AREA) } \\
\% \text { OF V (AREA) }\end{array}$ & $\begin{array}{l}9.8 \\
13.8 \\
71.7 \\
4.8\end{array}$ & & \\
\hline AREA ON M & & & & $\% \mathrm{~L} / \% \mathrm{C}$ & 1.40 & & \\
\hline
\end{tabular}

CHANGE IN LOS GATOS'S MAIN STREET FROM 1960 TO 1988

\begin{tabular}{|c|c|c|c|c|c|c|c|}
\hline $\begin{array}{l}\mathrm{CH} \text { IN \# C } \\
\mathrm{CH} \text { M2 OF C } \\
\text { CH AVE M2 OF C } \\
\mathrm{CH} \% \text { OF C AREA }\end{array}$ & $\begin{array}{l}-12 \\
-2136 \\
-22 \\
-12.465\end{array}$ & $\begin{array}{l}\text { CH IN \# L } \\
\text { CH M2 OF L } \\
\text { CH AVE M2 OF L } \\
C H \% \text { OF L AREA }\end{array}$ & $\begin{array}{l}15 \\
1420 \\
-22 \\
6.1\end{array}$ & $\begin{array}{l}\text { CH IN \# M } \\
\text { CH M2 OF M } \\
\text { CH AVE M2 OF M } \\
\text { CH \% OF M AREA }\end{array}$ & $\begin{array}{l}10 \\
2663 \\
10 \\
6.2\end{array}$ & $\begin{array}{l}\mathrm{CH} \mathrm{IN} \mathrm{\#} \mathrm{V} \\
\mathrm{CH} \text { M2 OF V } \\
\mathrm{CH} \% \text { OF V AREA }\end{array}$ & $\begin{array}{l}2 \\
-21 \\
-0.6\end{array}$ \\
\hline $\begin{array}{l}\text { CH TOTAL NBR } \\
\text { CH TOTAL M2 } \\
\text { CH AVE M2 ALL }\end{array}$ & $\begin{array}{l}15 \\
2104 \\
-5\end{array}$ & & & $\begin{array}{l}\% \mathrm{CH} \text { IN \% C } \\
\% \mathrm{CH} \text { IN \% L } \\
\% \mathrm{CH} \text { IN \% M } \\
\% \mathrm{CH} \text { IN \% V }\end{array}$ & $\begin{array}{l}-55.9 \\
78.7 \\
9.4 \\
-12.0\end{array}$ & & \\
\hline$\% \mathrm{CH}$ TOT M2 & 11.2 & & & $\mathrm{CH}$ LC & 1.05 & & \\
\hline
\end{tabular}


Table 6: CONVENIENCE STORES IN LOS GATOS IN 1988 BY STANDARD LAND USE CODE

\begin{tabular}{|c|c|c|c|c|c|c|}
\hline \multirow{2}{*}{$\begin{array}{l}\text { Name of Establishment } \\
\text { MRS. FIELD'S - cookies }\end{array}$} & \multirow{2}{*}{$\begin{array}{l}\text { Street } \\
51\end{array}$} & \multicolumn{2}{|l|}{ Address } & $\begin{array}{l}\text { Standard } \\
\text { Land Use } \\
\text { Code }\end{array}$ & Type & Area $M$ \\
\hline & & \multicolumn{2}{|c|}{ N. SANTA CRUZ AVE } & 546 & $\mathrm{C}$ & 91 \\
\hline BLACK WATCH - bar & 141.5 & \multicolumn{2}{|c|}{ N. SANTA CRUZ AVE } & 582 & C & 132 \\
\hline CARRY NATION'S - bar & 8 & \multicolumn{2}{|c|}{ N. SANTA CRUZ AVE } & 582 & C & 86 \\
\hline VIDEO ERA & 218 & \multicolumn{2}{|c|}{ N. SANTA CRUZ AVE } & 592 & C & 210 \\
\hline MAIN STREET VIDEO & 33 & \multicolumn{2}{|c|}{ E. MAIN STREET } & 599 & C & 147 \\
\hline FORD CLEANERS & 33 & \multicolumn{2}{|c|}{ N. SANTA CRUZ AVE } & 621 & C & 345 \\
\hline GATITO CLEANERS & 312 & \multicolumn{2}{|c|}{ N. SANTA CRUZ AVE } & 621 & C & 214 \\
\hline TIP TOP CLEANERS & 216 & \multicolumn{2}{|c|}{ N. SANTA CRUZ AVE } & 621 & $\mathrm{c}$ & 118 \\
\hline LAUNDRY MAT & 9 & \multicolumn{2}{|c|}{ MONTEBELLOWAY } & 621 & $\mathrm{C}$ & 116 \\
\hline SMART CLEANERS & 11 & \multicolumn{2}{|c|}{ MONTEBELLOWAY } & 621 & C & 116 \\
\hline PROFESSIONAL SERVICES - cleaners+alt. & 114 & \multicolumn{2}{|c|}{ ROYCE STREET } & 621 & C & 42 \\
\hline NEW REFLECTIONS - hair & 236 & \multicolumn{2}{|c|}{ N. SANTA CRUZ AVE } & 623 & C & 285 \\
\hline HAIR FASHION & 210 & \multicolumn{2}{|c|}{ N. SANTA CRUZ AVE } & 623 & C & 154 \\
\hline DIVA - beauty & 65 & \multicolumn{2}{|c|}{ W. MAIN STREET } & 623 & C & 103 \\
\hline FANTASTIC SAM'S - beauty & 347 & \multicolumn{2}{|c|}{ N. SANTA CRUZ AVE } & 623 & C & 98 \\
\hline OFF MAIN STREET HAIR SALON & 296 & \multicolumn{2}{|c|}{ N. SANTA CRUZ AVE } & 623 & $\mathrm{C}$ & 88 \\
\hline K. KRAKE - beauty & 359 & \multicolumn{2}{|c|}{ VILLAGE LANE } & 623 & $\mathrm{c}$ & 72 \\
\hline A LITTLE CORNER OF PARIS - beauty & 341 & \multicolumn{2}{|c|}{ VILLAGE LANE } & 623 & $-\mathrm{C}$ & 71 \\
\hline COURTYARD HAIR DESIGN & 59 & \multicolumn{2}{|c|}{ N. SANTA CRUZ AVE } & 623 & c & 52 \\
\hline PAVILION - beauty & 224.5 & \multicolumn{2}{|c|}{ N. SANTA CRUZ AVE } & 623 & C & 50 \\
\hline LOCAL TALENT - beauty & 359.5 & \multicolumn{2}{|c|}{ VILLAGE LANE } & 623 & C & 36 \\
\hline LEN'S BARBER SHOP & 114 & \multicolumn{2}{|c|}{ ROYCE STREET } & 623 & C & 25 \\
\hline MARIO'S SHOE REPAIR & 224 & \multicolumn{2}{|c|}{ N. SANTA CRUZ AVE } & 625 & C & 50 \\
\hline LOS GATOS NAILWORKS & 78 & \multicolumn{2}{|c|}{ W. MAIN STREET } & 629 & C & 88 \\
\hline PERFECT NAILS & 338.5 & \multicolumn{2}{|c|}{ VILLAGE LANE } & 629 & C & 72 \\
\hline CLASSIQUE NAILS & 336 & N. SAN & ITA CRUZ AVE & 629 & $\mathrm{C}$ & 63 \\
\hline CULTURED NAILS & 215 & N. SAN & ITA CRUZ AVE & 629 & c & 17 \\
\hline & $\begin{array}{l}\text { NUMBEF } \\
\text { M2 OF } \\
\text { AVE OF } \\
\% \text { OF C } \\
\text { SD FOR }\end{array}$ & $\begin{array}{l}R O F C \\
C \\
=C \\
\text { (AREA) } \\
C \text { A }\end{array}$ & $\begin{array}{l}27 \\
2941 \\
109 \\
9.9 \\
77\end{array}$ & & & \\
\hline
\end{tabular}

*** Establishment assigned approximate value for area 


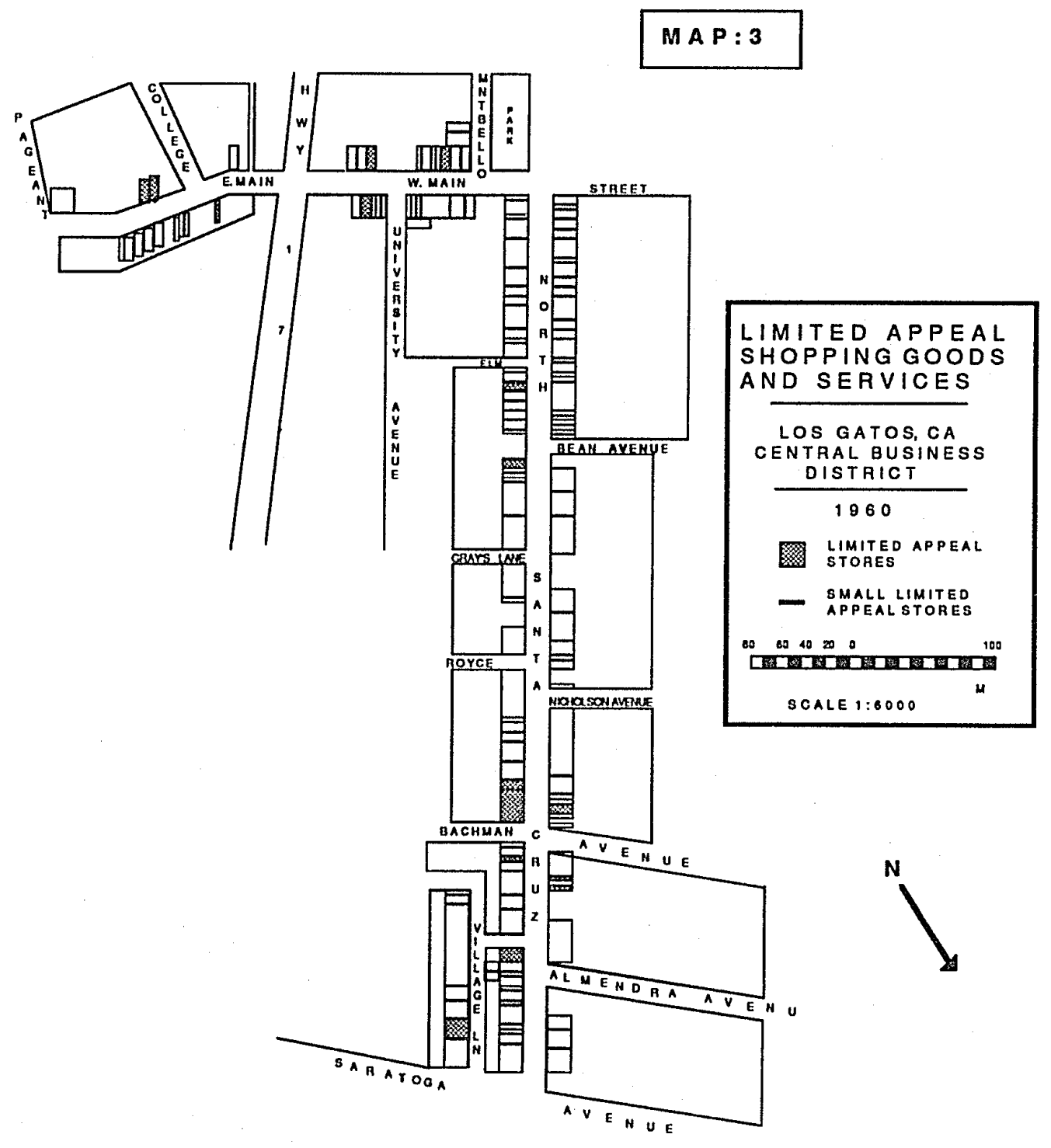


The percentage of limited appeal space tripled from 1960 to 1988. (see fig. 1) It grew from $8.3 \%$ to $24.6 \%$ of the total retail floorspace. The ratio of limited appeal space to convenience space increased dramatically from .36 to 2.48 . There are now two distinct clusters of limited appeal stores. The first is along the north side of W. Main Street, and the other is on the east side of Village Lane. (see map 4) The majority of these limited appeal stores are antique shops. In 1960, there were only two small antique stores which together consisted of $267 \mathrm{~m}^{2}$ of floorspace. By 1988, there were 14 antique stores consisting of 2240 $\mathrm{m} 2$ of floorspace. Antique stores now take up $30.7 \%$ of the total limited appeal space. (see tables 7 and 8 )

\section{Mass Appeal}

Almost two-thirds of the total retail space was used by mass appeal stores in 1960. (see map 5) No one type of mass appeal store dominates. Banks took up $5.7 \%$ of the space; various clothing stores took up $10.4 \%$; restaurants took up $8.3 \%$, and car dealerships took up $11.8 \%$. (see table 9) Mass appeal stores are located throughout the CBD. There is, however, a relative absence of mass appeal stores on Village and parts of Main Street, which by 1988 will be dominated by limited appeal stores. (see map 6)

Mass appeal stores took up two-thirds of the total retail space in 1988. (see fig. 1) This is a slight decrease in percentage from 1960. The percentage of retail space used by banks grew from $5.7 \%$ to $12.2 \%$. (see table 10) The percentage of space used by clothing stores grew from 
M A P : 4

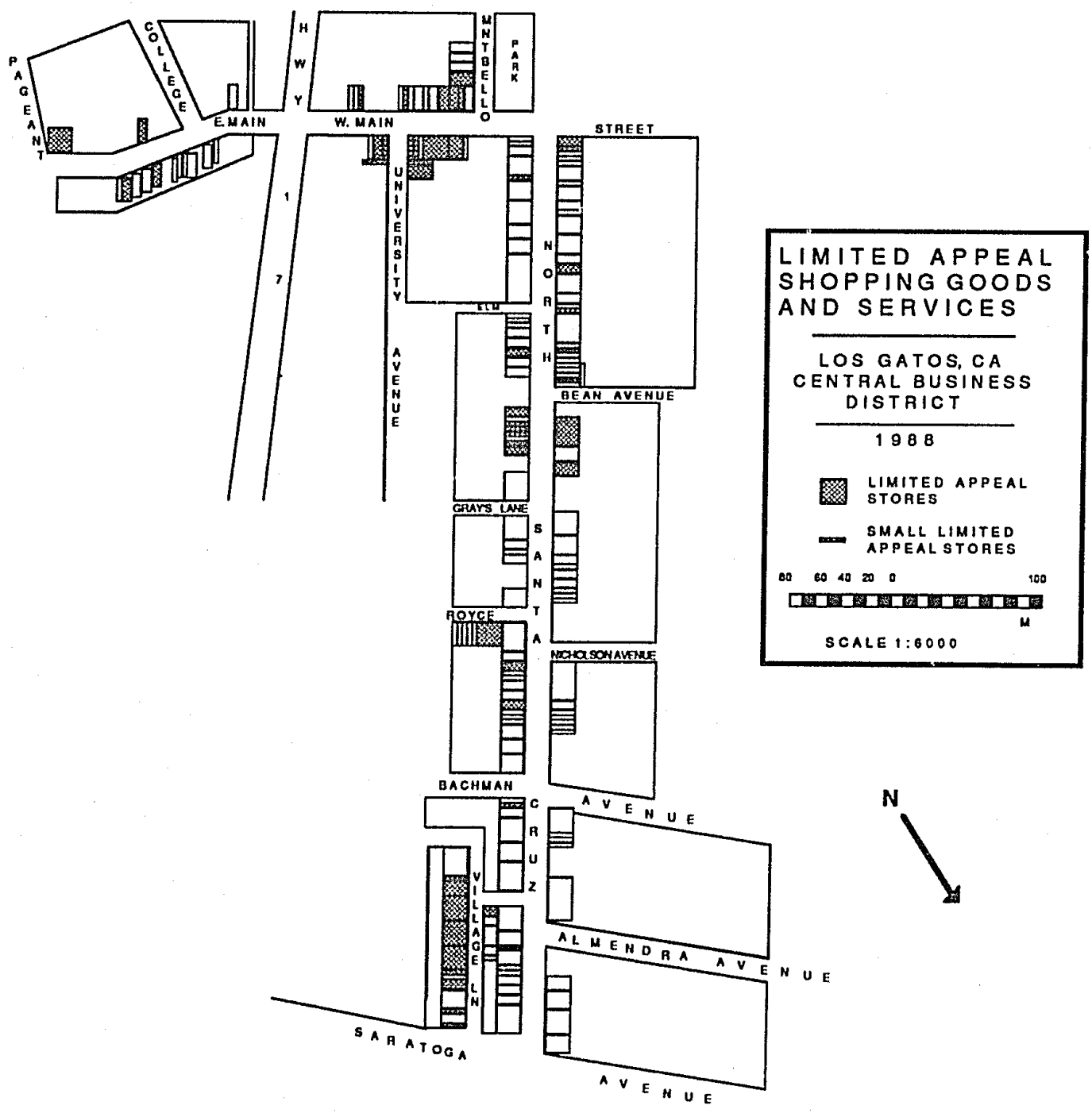


TABLE 7 : LIMITED APPEAL STORES IN LOS GATOS IN 1960 BY STANDARD LAND USE CODE

\begin{tabular}{|c|c|c|c|c|c|c|}
\hline \multirow{2}{*}{$\begin{array}{l}\text { Name of Establishement } \\
\text { HANSEN'S NATURAL FOODS }\end{array}$} & \multicolumn{3}{|c|}{ Street Address } & $\begin{array}{l}\text { Standard } \\
\text { Land Use } \\
\text { Code }\end{array}$ & Type & Area $M^{2}$ \\
\hline & 225 & N. SANTACI & SRUZ AVE & 541 & $L$ & 133 \\
\hline GINGER JAR ANTIQUES & 32 & E. MAIN STR & REET & 593 & $L$ & 169 \\
\hline BERG'S ANTIQUES & 303 & N. SANTA Cl & SRUZ AVE & 593 & L & 98 \\
\hline STEVEN'S MUSIC CENTER & 222 & N. SANTACI & SRUZ AVE & 594 & L & 103 \\
\hline VAULT, THE - art gallery & 68 & W. MAIN STI & TREET & 599 & L & 200 \\
\hline NERENBERGOPTOM & 54 & N. SANTA C & ZRUZ AVE & 599 & L & 166 \\
\hline NEOLITE CO SIGNS & 23 & E. MAIN STF & REET & 599 & L & 162 \\
\hline VILLAGE CAMERA SHOP & 318 & N. SANTA C & SRUZ AVE & 599 & L & 150 \\
\hline PIONEER CIGARSTORE & 109 & W. MAIN STI & REET & 599 & $L$ & 114 \\
\hline LITTLE VILLAGE NEEDLECRAFT STUDIO & 364 & VILLAGE LAI & NE & 599 & $L$ & 96 \\
\hline GINA'S GIFTS- boutique & 25 & W. MAIN STI & REET & 599 & L & 96 \\
\hline GOLDENBUDDHA GIFT SHOP & 307 & N. SANTA C & RUZ AVE & 599 & $L$ & 82 \\
\hline LOS GATOS PET SHOP & 98 & W. MAIN STI & REET & 599 & L & 36 \\
\hline RHODA'S TREASURE NOOK GIFTS & 284 & N. SANTAC & ZRUZ AVE & 599 & $\mathrm{~L}$ & 31 \\
\hline COLUEDON STUDIO PHOTOG & 236 & N. SANTACI & SRUZ AVE & 622 & $L$ & 304 \\
\hline COOK'S REPAIR SHOP SAWS AND TOOLS & 108 & N. SANTA Cl & SRUZ AVE & 649 & $L$ & 208 \\
\hline \multirow[t]{2}{*}{ QUICKWAY SAW FILER } & 34 & E. MAIN STR & REET & 699 & L & 40 \\
\hline & $\begin{array}{l}\text { NUME } \\
\text { TOTA } \\
\text { AVEI } \\
\% \text { OF } \\
\text { SDFC }\end{array}$ & $\begin{array}{l}\text { IBEROFL } \\
\text { AL M2 OF L } \\
\text { M2 OF L } \\
\text { F L (AREA) } \\
\text { ORL }\end{array}$ & $\begin{array}{l}17 \\
2188 \\
129 \\
8.3 \\
70\end{array}$ & & & \\
\hline
\end{tabular}


TABLE 8: LIMITED APPEAL STORES IN LOS GATOS IN 1988 BY STANDARD LAND USE CODE

Name of Establishement

FERRARI OF LOS GATOS

COTTONWORKS - boutique

AMY'S CUSTOM TAILORING

SUMI'S TAILORING AND ALTERATIONS

COSTUMES

CHART HOUSE - rest .

ANTIQUES COLONY

MAIN STREET EXCHANGE - antiques

ANTIQUES

BRANNDDY + OTERO ANTIQUES

ANTIQUES

ANTIQUES

JEAN NEWWARTS ANTIQUES

PATTERSON'S ANTIQUES

LES PIOSSON INTERIORS - antiques

MARIA'S OF LOS GATOS ANTIQUES

ANTIQUARIUM ANTIQUES

PRECIOUSOBJECTS GALLERY

DE VILLAR GALLERY - antiques

CONOVER - antiques

BARE ESCENTUALS - body lotions, cards 54

G. STEWART GEMOLOGIST + GOLDSMITH 3

WOLF COMPUTER

FAST FREDDY'S GAGS AND GIFTS

PICTURES AND FRAMES

STENCILHOUSE

A TOUCH OF BRASS

LOS GATOS OPTICAL - glasses, contacts 114

TRENT POTTERY

FRENCH CONNECTION - gifts

CORNER STORE, THE - KITCHEN'S

PUTTIN' ON THE RITZ

GATITO GAUERY

LOS GATOS YARN CO.

CATS SURF. SHOP

GINA'S - boutique

CONCOURSE ACCESSORIES - car things

ANGEL DE LAS FLORES- gifts

LOS GATOS BEAUTY OUTLET - makeup

BEARS IN THE WOOD - cards + bears

VILLAGE GARDEN - gifts

PURRSNICKETY

ANDRE'S PIPE GALLERY

326

9

137
Street Address

Standard Type Area $M^{2}$

Land Use

Code
66 E. MAIN STREET 551

49 N. SANTA CRUZ AVE 562

338.5 VILLAGE LANE 567

116 N. SANTA CRUZ AVE 567

338 VILLAGELANE 569

115 N. SANTA CRUZ AVE 581

140 W. MAIN STREET 593

150

344

348

3

334

110

88

110

112

120

39.5

98

45

W. MAIN STREET

VILLAGE LANE

VILLAGE LANE MONTEBELOWAY

593

593

593

VILLAGE LANE

$W$. MAIN STREET

W. MAIN STREET

593

593

593

593

N. SANTA CRUZ AVE 593

N. SANTA CRUZ AVE 593

W. MAIN STREET 593

N. SANTA CRUZ AVE 593

W. MAIN STREET 593

W. MAIN STREET 593

N. SANTA CRUZ AVE 596

N. SANTA CRUZ AVE 597

N. SANTA CRUZ AVE 599

N. SANTA CRUZ AVE 599

N. SANTA CRUZ AVE 599

VILLAGE LANE 599

N. SANTA CRUZ AVE 599

ROYCESTREET 599

N. SANTA CRUZ AVE 599

N. SANTA CRUZ AVE 599

N. SANTA CRUZ AVE 599

UNIVERSTTY AVE $\quad 599$

W. MAIN STREET $\quad 599$

W. MAIN STREET 599

N. SANTA CRUZ AVE 599

W. MAIN STREET 599

N. SANTA CRUZ AVE 599

318.5 N. SANTA CRUZ AVE 599

305 N. SANTA CRUZ AVE 599

59 N. SANTA CRUZ AVE 599 VILLAGELANE $\quad 599$ UNIVERSITY AVE 599 W. MAIN STREET 599

$\begin{array}{ll}\mathrm{L} & 897 \\ \mathrm{~L} & 91 \\ \mathrm{~L} & 36 \\ \mathrm{~L} & 35 \\ \mathrm{~L} & 36 \\ \mathrm{~L} & 229 \\ \mathrm{~L} & 655 \\ \mathrm{~L} & 329 \\ \mathrm{~L} & 210 \\ \mathrm{~L} & 210 \\ \mathrm{~L} & 169 \\ \mathrm{~L} & 140 \\ \mathrm{~L} & 128 \\ \mathrm{~L} & 91 \\ \mathrm{~L} & 84 \\ \mathrm{~L} & 60 \\ \mathrm{~L} & 57 \\ \mathrm{~L} & 43 \\ \mathrm{~L} & 36 \\ \mathrm{~L} & 28 \\ \mathrm{~L} & 166 \\ \mathrm{~L} & 47 \\ \mathrm{~L} & 440 \\ \mathrm{~L} & 215 \\ \mathrm{~L} & 210 \\ \mathrm{~L} & 210 \\ \mathrm{~L} & 208 \\ \mathrm{~L} & 208 \\ \mathrm{~L} & 193 \\ \mathrm{~L} & 170 \\ \mathrm{~L} & 162 \\ \mathrm{~L} & 123 \\ \mathrm{~L} & 120 \\ \mathrm{~L} & 114 \\ \mathrm{~L} & 99 \\ \mathrm{~L} & 96 \\ \mathrm{~L} & 82 \\ \mathrm{~L} & 71 \\ \mathrm{~L} & 70 \\ \mathrm{~L} & 61 \\ \mathrm{~L} & 57 \\ \mathrm{~L} & 54 \\ \mathrm{~L} & 53\end{array}$




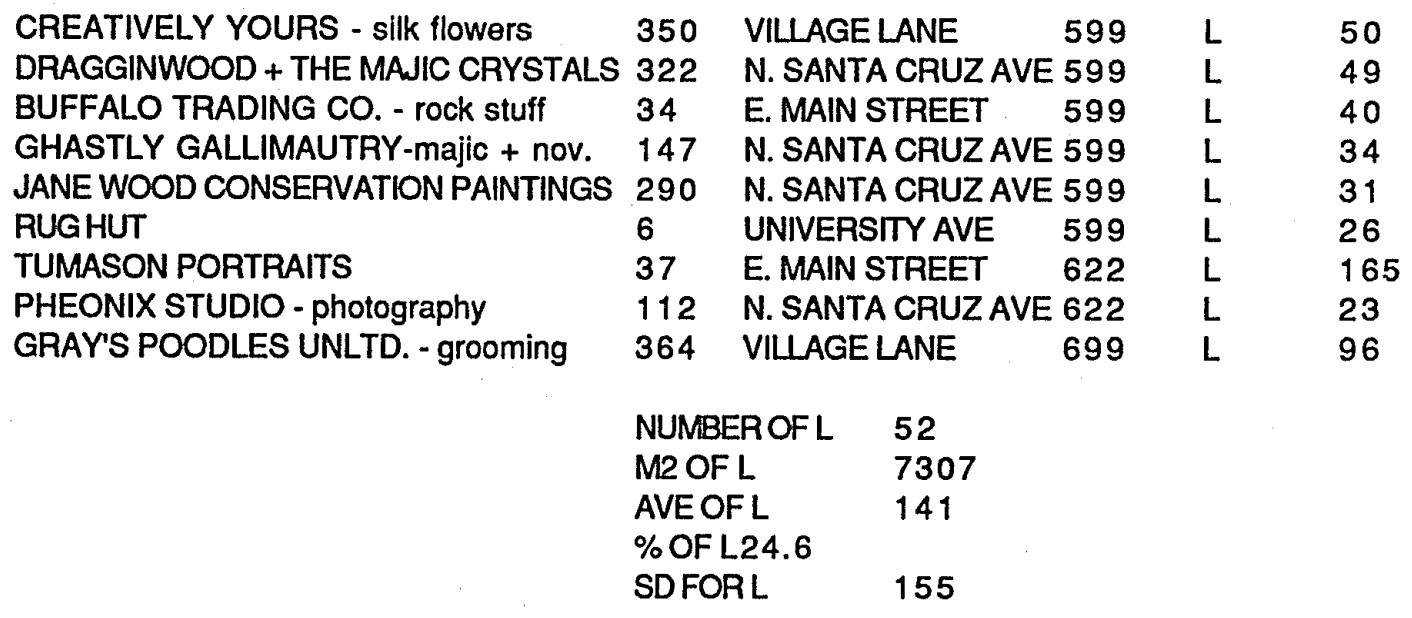

*** Establishment assigned approximate value for area. 
M A P : 5

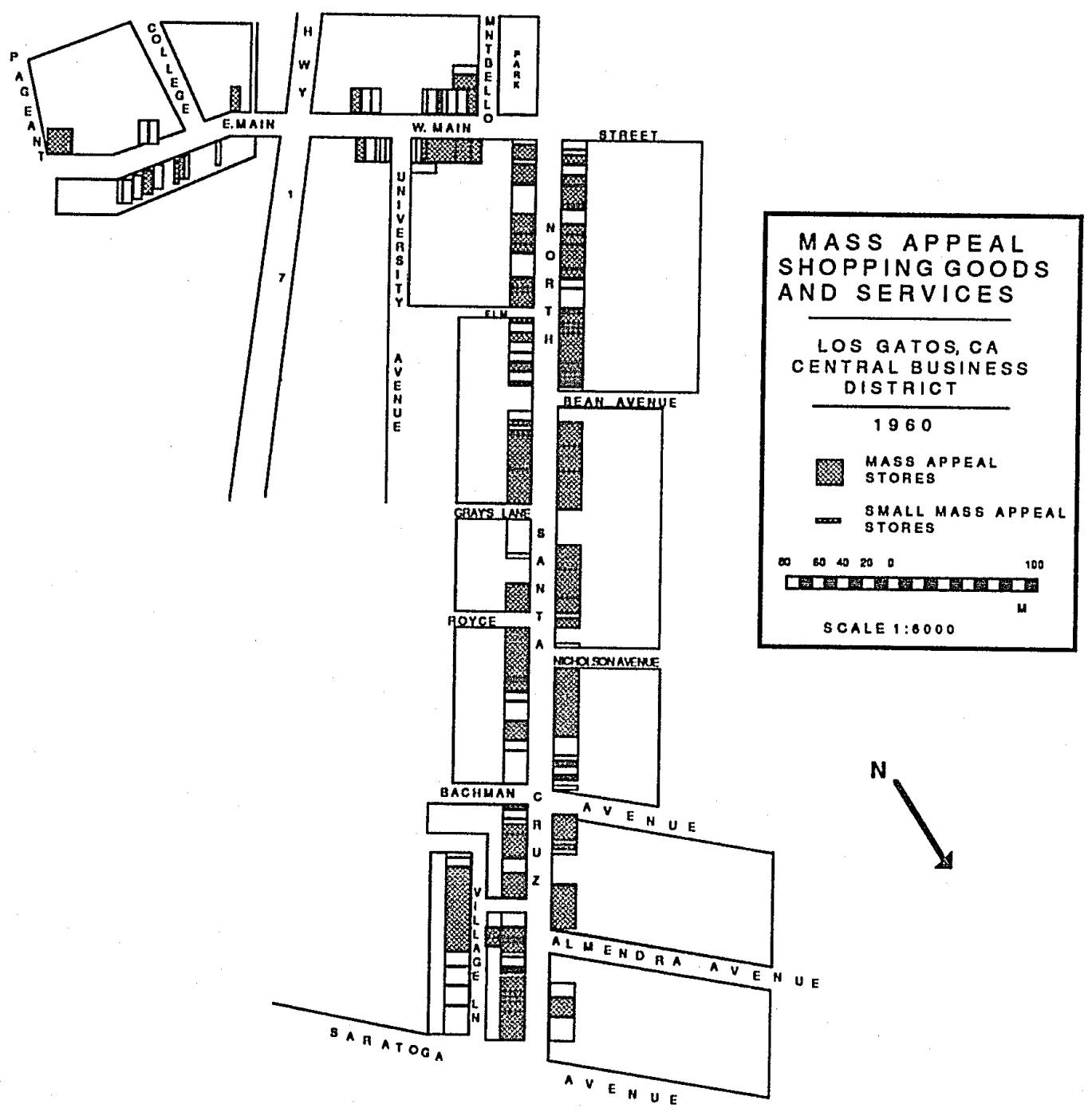


TABLE 9 : MASS APPEAL STORES IN LOS GATOS IN 1960 BY STANDARD LAND USE CODE

\begin{tabular}{|c|c|c|c|c|c|}
\hline \multirow{2}{*}{$\begin{array}{l}\text { Name of Establishement } \\
\text { WESTERN GREYHOUND LNES }\end{array}$} & \multirow{2}{*}{$\begin{array}{l}\text { Street } \\
145\end{array}$} & Address & $\begin{array}{l}\text { Standard } \\
\text { Land Use } \\
\text { Code }\end{array}$ & Type & Area $M$ \\
\hline & & N. SANTA CRUZ AVE & 421 & $M$ & 9 \\
\hline TRAVEL ADVISORS & 56 & N. SANTA CRUZ AVE & 492 & M & \\
\hline BERRYMAN + SON PLUMBING & 32 & N. SANTA CRUZ AVE & 522 & M & \\
\hline DIDUCA BROS INC PLUMBING & 60 & W. MAIN STREET & 522 & M & \\
\hline SORENSONPLMB & 21 & W. MAIN STREET & 522 & M & \\
\hline LOS GATOS PAINT + WALL PAPER *** & 131 & N. SANTA CRUZZ AVE & 523 & M & 237 \\
\hline LOS GATOS HARDWARE INC & 150 & W. MAIN STREET & 525 & $\mathbf{M}$ & 329 \\
\hline LOS GATOS EQUIP + SUPP & 111 & N. SANTA CRUZ AVE & 525 & M & 233 \\
\hline TEMPLEMAN HDW & 24 & N. SANTA CRUZ AVE & 525 & M & 208 \\
\hline CHRISLOWS DEPT STORE & 201 & N. SANTA CRUZ AVE & 531 & M & 945 \\
\hline SPROUSE REITZ CO. - variety store & 25 & N. SANTA CRUZ AVE & 533 & $M$ & \\
\hline RAO'S SUPER MKT & 206 & N. SANTA CRUZ AVE & 541 & M & 563 \\
\hline S SWEET SHOP & 39 & N. SANTA CRUZ AVE & 544 & M & 98 \\
\hline ADE CANDIES & 39.5 & N. SANTA CRUZ AVE & 544 & $M$ & 43 \\
\hline MPASTRY SHOP & 55 & RUZ AVE & 546 & M & 91 \\
\hline SIMCA CAR DLR & 66 & E. MAIN STREET & 551 & M & 897 \\
\hline ACOLDSMOBILE & 331 & N. SANTA CRUZ AVE & 551 & M & 642 \\
\hline FRASER MILT PONTIAC CO - used cars & 127 & N. SANTA CRUZ AVE & 551 & M & 412 \\
\hline LOS GATOS AUT & 122 & CRUZ AVE & 552 & M & 573 \\
\hline WES & 11 & N. SANTA CRUZ AVE & 552 & M & 279 \\
\hline W.J. MEN'S & 16 & N. SANTA CRUZ AVE & 561 & $M$ & 84 \\
\hline FOR MEN & 336 & ZAVE & 561 & 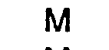 & 63 \\
\hline JAY VEE DRESS SHOP & 220 & JZAVE & 562 & M & 210 \\
\hline GLADYS ANN SHOP - women's clothes & 33 & E. MAIN STREET & 562 & M & 147 \\
\hline NELL + MINA'S DRESS SHOP & 19 & N. SANTA CRUZ AVE & 562 & $M$ & 145 \\
\hline VE WOMEN'S CLOTHES & 320 & N. SANTA CRUZ AVE & 562 & $M$ & 125 \\
\hline n's clothes & 51 & N. SANTA CRUZ AVE & 562 & M & 91 \\
\hline CARME & 53 & JZAVE & 562 & M & 91 \\
\hline OTHES & 296 & Z AVE & 562 & $M$ & 88 \\
\hline WILLIAMS MRS SHOPPE & 130 & W. MAIN & 562 & . & 78 \\
\hline CHILDREN'S SHOP THE & 9 & N. SANTA CRUZ AVE & 564 & M & 113 \\
\hline HOP - children's clothes & 348 & N. SANTA CRUZ AVE & 564 & M & 26 \\
\hline IONS LTD & 47 & N. SANTA CRUZ AVE & 565 & M & 91 \\
\hline FELIC & 137 & N. SANTA CRUZ AVE & 566 & $M$ & 132 \\
\hline RY SHOE DLRS & 324 & N. SANTA CRUZ AVE & 566 & $M$ & 127 \\
\hline CORDONASHOES & 214 & N. SANTA CRUZ AVE & 566 & $M$ & 112 \\
\hline ANTHONY THE TAILOR & 359 & VILAGE LANE & 567 & $M$ & 108 \\
\hline SOMMER'S FURN & 140 & W. MAIN STREET & 571 & M & 655 \\
\hline LOS GATOS THEATRE & 41 & N. SANTA CRUZ AVE & 571 & $M$ & 500 \\
\hline MICKELSEN'S FURN CO INC. & 301 & N. SANTA CRUZ AVE & 571 & M & 333 \\
\hline VILAGE FL COVERINGS & 160 & W. MAIN STREET & 571 & $M$ & 195 \\
\hline MADSEN'S LINEOLEUM & 3 & MONTEBELO WAY & 571 & $M$ & 169 \\
\hline FINNS WALLPAPERS & 242 & N. SANTA CRUZ AVE & 571 & M & 77 \\
\hline LOS GATOS SHADE SHOP & 14 & E. MAIN STREET & 571 & $M$ & 66 \\
\hline
\end{tabular}




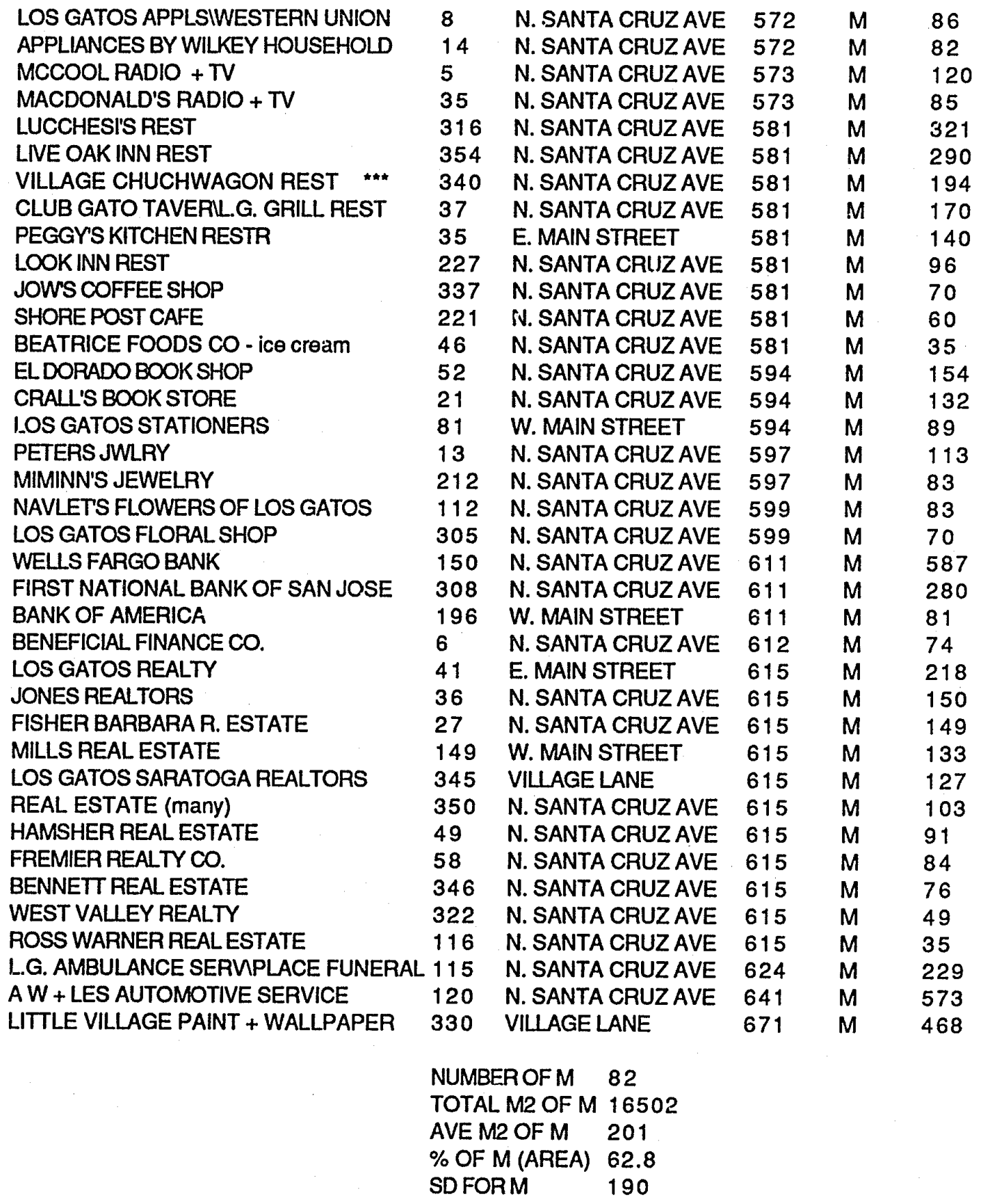

*** Establishment assigned approximate value for area. 
M A P : 6

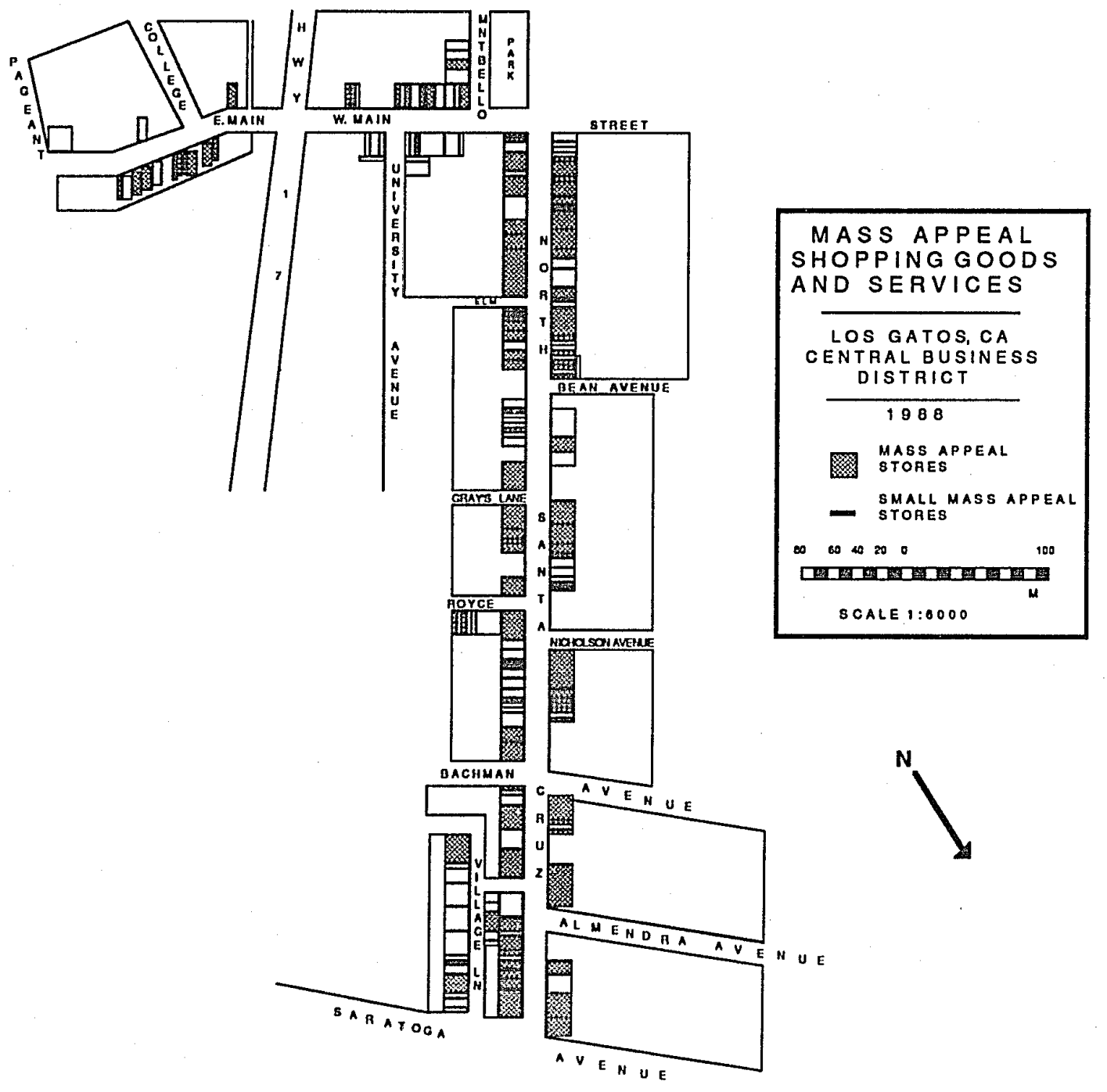


TABLE 10: MASS APPEAL STORES IN LOS GATOS IN 1988 BY STANDARD LAND USE CODE

\begin{tabular}{|c|c|c|c|c|c|}
\hline Name of Establishement & Street & t Address & $\begin{array}{l}\text { tandard } \\
\text { and Use } \\
\text { ode }\end{array}$ & ype & Area \\
\hline TRAVEL ADVISORS & 56 & N. SANTA CRUZ AVE & 492 & $M$ & 166 \\
\hline TRAVEL STORE & 56.5 & N. SANTA CRUZ AVE & 492 & $\mathbf{M}$ & 166 \\
\hline FINNS WALLPAPERS & 242 & N. SANTA CRUZ AVE & 523 & M & 77 \\
\hline LOS GATOS SHADE SHOP & 14 & E. MAIN STREET & 523 & $\mathbf{M}$ & 66 \\
\hline RURAL SUPPLY - ace hardware & 110 & S. SANTA CRUZ AVE & 525 & $M$ & 484 \\
\hline LE BOULANGER - bakery & 133 & N. SANTA CRUZ AVE & 546 & $M$ & 306 \\
\hline GRUMB'S - bakery & 81 & W. MAIN STREET & 546 & $M$ & 89 \\
\hline ANRIENNE'S - women's clothes & 130 & N. SANTA CRUZ AVE & 562 & M & 619 \\
\hline IDAMINA'S FASHIONS & 11 & N. SANTA CRUZ AVE & 562 & M & 279 \\
\hline IDAMINA'S FASHIONS & 17 & N. SANTA CRUZ AVE & 562 & $M$ & 248 \\
\hline COTTON CLUB - clothes & 52 & N. SANTA CRUZ AVE & 562 & $\mathbf{M}$ & 154 \\
\hline SCOFFE + LUS PINK PETTICOAT & 320 & N. SANTA CRUZ AVE & 562 & $M$ & 125 \\
\hline DESIGN MINE FASHION BOUTIQUE & 307 & N. SANTA CRUZ AVE & 562 & $\mathbf{M}$ & 82 \\
\hline NUANCE OF LOS GATOS - clothes & 130 & W. MAIN STREET & 562 & M & 78 \\
\hline VIDA CLOTHES & 48 & N. SANTA CRUZ AVE & 562 & $\mathbf{M}$ & 70 \\
\hline L.F. ELEGANCE - women's clothes & 114 & ROYCESTREET & 562 & $\mathbf{M}$ & 42 \\
\hline FASHIONS AND EXCESSORIES & 42 & N. SANTA CRUZ AVE & 562 & $\mathbf{M}$ & 35 \\
\hline METRO - large women's clothes & 55 & W. MAIN STREET & 562 & $\mathbf{M}$ & 28 \\
\hline SONA'S BOUTIQUE - women's clothes & 114 & ROYCESTREET & 562 & $\mathbf{M}$ & 25 \\
\hline CHILDREN'S WORLD & 301 & N. SANTA CRUZ AVE & 564 & $M$ & 333 \\
\hline CAT + THE FIDDLE - child. clothes & 139 & N. SANTA CRUZ AVE & 564 & $\mathbf{M}$ & 78 \\
\hline CHRISTOW'S - clothes & 201 & N. SANTA CRUZ AVE & 565 & M & 693 \\
\hline BENELLON LOS GATOS - clothes & 23 & N. SANTA CRUZ AVE & 565 & $M$ & 370 \\
\hline CHRISTOWS CLOTHESOUT CENTER & 201 & N. SANTA CRUZ AVE & 565 & M & 186 \\
\hline PERIN'S - clothes & 35 & W. MAIN STREET & 565 & M & 28 \\
\hline SCOTTS SHOES & 324 & N. SANTA CRUZ AVE & 566 & $M$ & 127 \\
\hline WALKING EXPERIENCE - shoes & 345 & VILLAGE LANE & 566 & M & 127 \\
\hline PEACOCK - shoes & 330 & N. SANTA CRUZ AVE & 566 & M & 62 \\
\hline CALIFORNIA INTERIORS & 25 & N. SANTA CRUZ AVE & 571 & M & 365 \\
\hline MARTINOS - furniture & 111 & N. SANTA CRUZ AVE & 571 & $\mathbf{M}$ & 233 \\
\hline LOS GATOS PORCH - furniture & 24 & N. SANTA CRUZ AVE & 571 & $\mathbf{M}$ & 208 \\
\hline LOS GATOS PORCH - furniture 2 & 32 & N. SANTA CRUZ AVE & 571 & M & 181 \\
\hline MAIDS QUARTERS & 36 & N. SANTA CRUZ AVE & 571 & $M$ & 150 \\
\hline ALTERNATING CURRENTS - lamps & 149 & W. MAIN STREET & 571 & $M$ & 133 \\
\hline CALIFORNIA INTERIORS & 21 & N. SANTA CRUZ AVE & 571 & M & 132 \\
\hline DECOR - lamps & 214 & N. SANTA CRUZ AVE & 571 & M & 112 \\
\hline WESTERN'S TV AND STEREO & 122 & N. SANTA CRUZ AVE & 573 & $M$ & 573 \\
\hline GOOD EARTH, THE - rest & 206 & N. SANTA CRUZ AVE & 581 & M & 563 \\
\hline MANDARIN DELIGHT - rest & 140 & N. SANTA CRUZ AVE & 581 & M & 321 \\
\hline PEDRO'S - rest & 316 & N. SANTA CRUZ AVE & 581 & M & 321 \\
\hline VILLAGE HOUSE + GARDEN - rest, gifts & 320 & VILLAGE LANE & 581 & M & 300 \\
\hline FLORILLO'S ITALIAN CUISINE & 354 & N. SANTA CRUZ AVE & 581 & $M$ & 290 \\
\hline DOROTHY'S GOURMET PIZZA & 25 & E. MAIN STREET & 581 & $\mathbf{M}$ & 180 \\
\hline
\end{tabular}




\begin{tabular}{|c|c|c|c|c|c|}
\hline PEASANT CHEF, THE - rest & 368 & VIШAGE LANE & 581 & $M$ & 180 \\
\hline HIGH-THAI - rest & 27 & E. MAIN STREET & 581 & $M$ & 165 \\
\hline POLCE SPAZIO - ice cream & 29 & E. MAIN STREET & 581 & $\mathbf{M}$ & 154 \\
\hline CHARMPS ELYSEES FRENCH CUISINE & 27 & N. SANTA CRUZ AVE & 581 & M & 149 \\
\hline MARTINELLI'S - rest, deli & 19 & N. SANTA CRUZAVE & 581 & $M$ & 145 \\
\hline SOUTHERN KITCHEN - reSt & 35 & E. MAIN STREET & 581 & M & 140 \\
\hline DOUBLE RAINBOW - icecream & 137 & N. SANTA CRUZ AVE & 581 & M & 132 \\
\hline ROUND TABLE PIZZA & 57 & N. SANTA CRUZ AVE & 581 & M & 115 \\
\hline LOS GATOS CAFE & 344 & N. SANTA CRUZ AVE & 581 & $M$ & 113 \\
\hline LOS GATOS COFFEE ROASTING CO. & 91 & W. MAIN STREET & 581 & M & 100 \\
\hline MABEL'S LANTERN HOUSE - rest & 39 & N. SANTA CRUZ AVE & 581 & M & 98 \\
\hline LA MAISON CROISSANT-deli & 303 & N. SANTA CRUZ AVE & 581 & M & 98 \\
\hline MAIN COURSE, THE - rest & 49 & E. MAIN STREET & 581 & $M$ & 95 \\
\hline GIUEY'S COFFEE SHOP & 47 & N. SANTA CRUZ AVE & 581 & M & 91 \\
\hline YOGURT CREATIONZ & 151 & N. SANTA CRUZ AVE & 581 & $\mathbf{M}$ & 83 \\
\hline ANDALE TAQUERIA - rest & 6 & N. SANTA CRUZ AVE & 581 & M & 74 \\
\hline LEXINGTON HOUSE - rest & 337 & N. SANTA CRUZ AVE & 581 & M & 70 \\
\hline SHUSHION THE RUN - rest & 114 & N. SANTA CRUZ AVE & 581 & $M$ & 60 \\
\hline MMMM MICHAELE'S - deli & 221 & N. SANTA CRUZ AVE & 581 & M & 60 \\
\hline CROWNBOOKS & 37 & N. SANTA CRUZ AVE & 594 & M & 170 \\
\hline CHANTICLEER - children's books & 23 & E. MAIN STREET & 594 & M & 162 \\
\hline HALLMARK - cards & 9 & N. SANTA CRUZ AVE & 594 & M & 113 \\
\hline AQUARIAN TAPES + BOOKS & 53 & N. SANTA CRUZ AVE & 594 & M & 91 \\
\hline CURIOUS BOOK SHOPPE & 198 & W. MAIN STREET & 594 & M & 68 \\
\hline MAIN STREET PAPERY & 23 & W. MAIN STREET & 594 & M & 65 \\
\hline ALEXX'S SPORTS STOP & 12 & N. SANTA CRUZ AVE & 595 & M & 245 \\
\hline BICYCLE SHOP & 41 & E. MAIN STREET & 595 & $M$ & 218 \\
\hline JEMERS BENCH & 236 & N. SANTA CRUZ AVE & 597 & $M$ & 285 \\
\hline GOLDSTON JEWELAY INC. & 50 & N. SANTA CRUZ AVE & 597 & M & 154 \\
\hline DETAGLE - jewelry & 13 & N. SANTA CRUZ AVE & 597 & $\mathbf{M}$ & 113 \\
\hline MORTON JEWELERS & 212 & N. SANTA CRUZ AVE & 597 & M & 83 \\
\hline YELLOW BRICK ROAD - jewelry & 346 & N. SANTA CRUZ AVE & 597 & M & 76 \\
\hline PAULS MISTER JEWLERS & 46 & N. SANTA CRUZ AVE & 597 & M & 35 \\
\hline DOMUS - kitchen supplies, gifts & 40 & N. SANTA CRUZ AVE & 599 & M & 600 \\
\hline ACCROSS THE BRIDGE - plants & 45 & E. MAIN STREET & 599 & $\mathbf{M}$ & 240 \\
\hline JUST CLOSETS & 134 & N. SANTA CRUZ AVE & 599 & M & 211 \\
\hline BOX, THE - cards, gifts & 138 & N. SANTA CRUZ AVE & 599 & M & 125 \\
\hline TEX'S TOYS TOO & 59 & N. SANTA CRUZ AVE & 599 & $M$ & 110 \\
\hline NAVLETS FLORIST & 222 & N. SANTA CRUZ AVE & 599 & M & 103 \\
\hline I'D RATHER KNOT - plants & 151 & N. SANTA CRUZ AVE & 599 & M & 83 \\
\hline FRAMECOMPANY, THE & 354 & VILLAGE LANE & 599 & $M$ & 50 \\
\hline NATURE'S ALLEY - plants & 108 & N. SANTA CRUZ AVE & 599 & M & 41 \\
\hline LUGGAGE CENTER & 59 & N. SANTA CRUZ AVE & 599 & M & 37 \\
\hline BANK OF AMERICA & 333 & N. SANTA CRUZ AVE & 611 & M & 642 \\
\hline WELLSFARGO BANK & 150 & N. SANTA CRUZ AVE & 611 & M & 587 \\
\hline DOWNEY SAVINGS & 127 & N. SANTA CRUZ AVE & 611 & M & 412 \\
\hline EUREKAFEDERAL SAVINGS & 236 & N. SANTA CRUZ AVE & 611 & M & 320 \\
\hline BANK OF THE WEST & 308 & N. SANTA CRUZ AVE & 611 & M & 280 \\
\hline CALDWELL BANKERS F & 333 & N. SANTA CRUZ AVE & 615 & M & 306 \\
\hline FOX + CARSKATON REALE & 350 & $\begin{array}{l}\text { N. SANTA CRUZ AVE } \\
39\end{array}$ & 615 & M & 103 \\
\hline
\end{tabular}


REALTY

VILLA REALTY

LONCHAR AND CO REALTORS

VILLA REALTY

SPEEDYFOTO

TV + VIDEO SERVICE - repair

LOS GATOS THEATRE

\begin{tabular}{|c|c|c|c|c|c|}
\hline 7 & \multicolumn{2}{|c|}{ MONTEBELLO WAY } & 615 & $M$ & 102 \\
\hline 201 & N. SANT & CRUZ AVE & 615 & $M$ & 33 \\
\hline 201 & N. SANT & CRUZ AVE & 615 & $M$ & 33 \\
\hline 201 & N. SANT & CRUZ AVE & 615 & M & 33 \\
\hline 347 & N. SANTA & CRUZ AVE & 622 & M & 120 \\
\hline 23.5 & W. MAIN & TREET & 649 & $M$ & 41 \\
\hline 41 & N. SANT & CRUZ AVE & 721 & $M$ & 500 \\
\hline NUME & 3EROF M & $\begin{array}{l}100 \\
18317\end{array}$ & & & \\
\hline M2O & $F M$ & 18317 & & & \\
\hline $\begin{array}{l}\text { AVEC } \\
\% \text { OF }\end{array}$ & PM & $\begin{array}{l}183 \\
61.6\end{array}$ & & & \\
\hline SD of & $F M$ & 156 & & & \\
\hline
\end{tabular}

7 MONTEBELLOWAY $615 \quad M \quad 102$

347 N. SANTA CRUZ AVE 622 M 120

23.5 W. MAIN STREET $649 \quad M \quad 41$

41 N. SANTA CRUZ AVE 721 M 500

*** Establishment assigned approximate value for area. 
$10.4 \%$ to $20.7 \%$ of the total mass appeal space. Restaurants grew even more dramatically from $8.3 \%$ of the mass appeal space to $22.4 \%$. Los Gatos is the only city in the study where real estate agency space increased in the percentage of the total mass appeal space: from $3.3 \%$ to $7.4 \%$. There are no mass appeal car dealerships left within the CBD because they tend to need a large amount of space which would be prohibitively expensive. The only car dealership left within the CBD has changed to a limited appeal establishment.

\section{The Main Street}

In $1960,71.4 \%$ of the total downtown floorspace was located on $\mathrm{N}$. Santa Cruz Avenue between Saratoga Avenue and West Main Street. This section of N. Santa Cruz Avenue constitutes the downtown "main street" of Los Gatos. The average store is 179 square meters, slightly smaller than the average for the whole downtown. The main street had a smaller percentage of limited appeal space but a slightly larger percentage of convenience space than the downtown as a whole. There was a slightly larger percentage of mass appeal floorspace on the "main street", as well as vacant retail space. The ratio of limited appeal space to convenience store space on the "main street" is almost identical the ratio for the downtown as a whole: .35 compared to .36 .

As expected, the composition of the main street differs from the downtown as a whole in that there is a greater percentage of mass appeal goods and a smaller percentage of limited and convenience space. (see fig.

1) The ratio of limited appeal space to convenience space grew 
considerably on the "main street", although not as much as the ratio did for the entire CBD, from .35 to 1.41 .

\section{Dther Changes}

Not only has the type of the stores changed, but the character of the stores has also changed. There are more boutiques among the mass appeal clothing stores. They now have names like "Design Mine Fashion Boutique", "Nuance of Los Gatos", and "L. F. Elegance". Los Gatos has become a place to go on the weekends to shop, dine, and browse. As stated above, there was a huge increase in restaurants. These restaurants have also taken on a higher class appeal. Instead of Peggy's Kitchen and Joe's coffee shop, they are now Dorothy's Gourmet Pizza and Champs Elysees French Cuisine. (see tables 9 and 10) The downtown pedestrian traffic seems to increase greatly on the weekends, but this question is left for another study. Much of the retail in the CBD no longer caters to the everyday needs of the residents. 


\section{SANTA CRUZ}

\section{$1960-1988$}

Santa Cruz in located on the Pacific Ocean, linked to San Jose by Highway 17. It is the largest city in its county. The city includes the University of California at Santa Cruz. It has a large local market but also caters to seasonal tourism and weekend shopping and dining.

Santa Cruz's Central Business District consists of the whole length of Pacific Avenue. It extends perpendicular to Pacific Avenue from Cedar Street to Front Street, including both sides of the streets. (see map 7) To the west of the downtown, there is a residential area. There is also a residential area to the south, but it is higher in elevation than the downtown is and somewhat cut off from easy access to the CBD. A river lies just northeast of the CBD.

In 1960 , Santa Cruz had a population of 25,600 . Although the population was twice that of the other cities in the study, the size of the CBD is similar to the size of the other CBDs. Santa Cruz had 53,866 square meters of retail floorspace, compared to $26,228 \mathrm{~m}^{2}$ for Los Gatos, and $55,719 \mathrm{~m}^{2}$ for Watsonville. The relative smallness of Santa Cruz's CBD is explained by the large retail area outside of the CBD. Only $39 \%$ of the retail stores in Santa Cruz were located in the CBD in 1960. (see table 2)

From 1960 to 1987 the population of Santa Cruz grew by $83 \%$, to 46,900 residents. By 1988 , the CBD floorspace had grown by $46.2 \%$. Much of this growth took place off the "main street". Santa Cruz has 


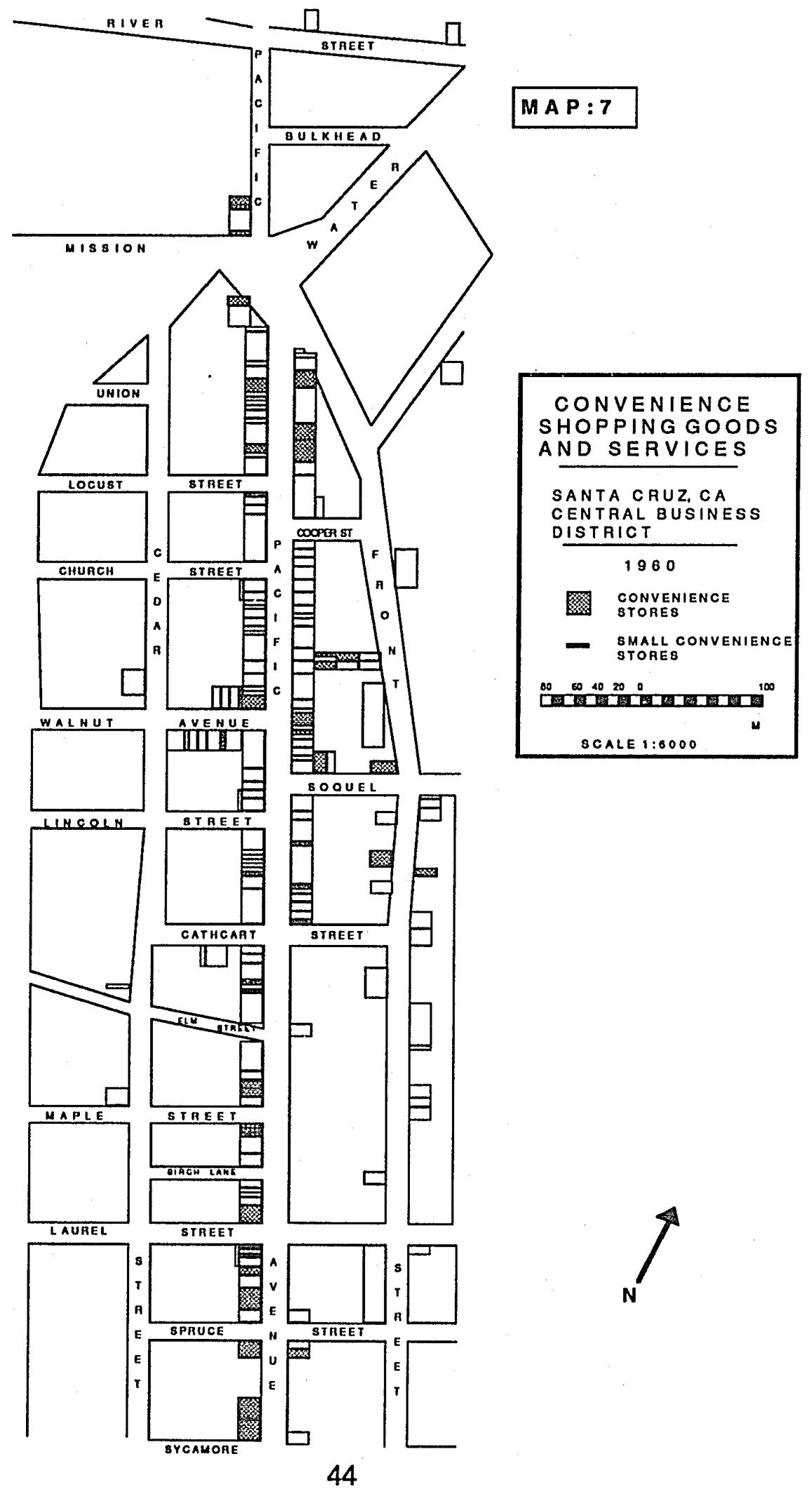


experienced both a quantitative and qualitative change in its Central Business District.

\section{Convenience Space}

In 1960 , convenience stores took up $13.1 \%$ of the total retail space. (see fig. 2; tables 11 and 12) The largest percentage of convenience space, $20.3 \%$, was used by bars and taverns. The next largest percentage of space was used by drug stores (14.6\%), corner markets (14.5\%), and liquor stores (10.9\%). (see table 12) At first it seems surprising that there were only three convenience stores on Front Street. (see map 7) Then one notices the river to the northeast of the downtown separates Front Street from the residential area beyond.

By 1988 , only $5.0 \%$ of the retail space was left in convenience goods. (see map 8) Convenience stores decreased in size, as well as in number. The remaining convenience stores have moved toward the periphery of the downtown. The only new type of convenience store in Santa Cruz is photocopying. The introduction of photocopying stores is most likely due to the large student population. There are no video stores in Santa Cruz's downtown, indicating that downtown Santa Cruz is no longer a convenience shopping area. Bars have the largest percentage of convenience space, $38.4 \%$. This is followed by liquor stores at $19.6 \%$, and beauty/barber shops at $10.6 \%$ of the total convenience space. (see table 13) 
Fig. 2

Percentages of retail land use in the CBD

\section{SANTA CRUZ}

SANTA CRUZ 1960

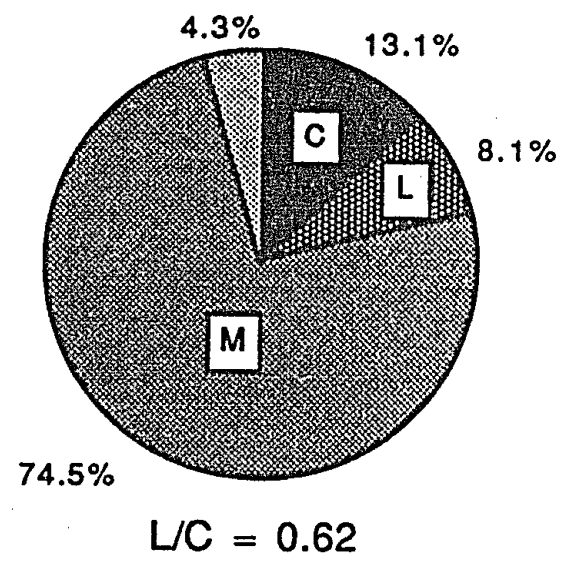

SANTACRUZ 1988

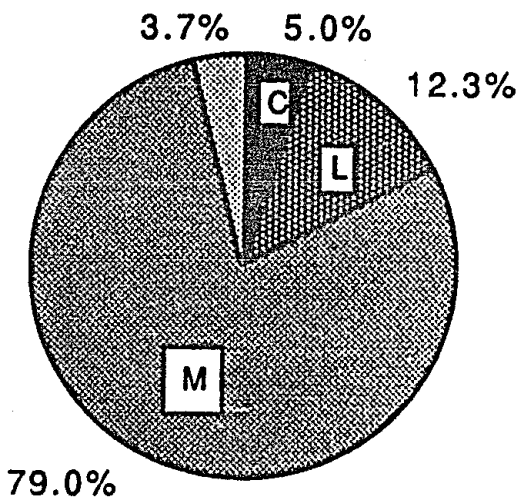

$$
L / C=2.46
$$

SANTA CRUZ 1960 THE MAIN STREET ONLY

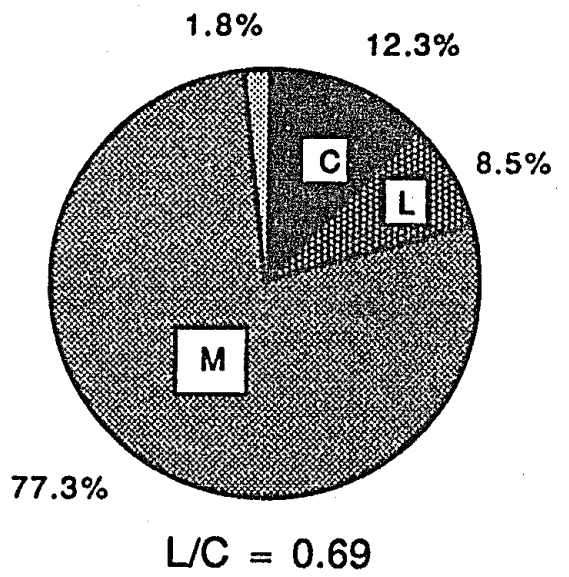

SANTA CRUZ 1988 THE MAIN STREET ONLY

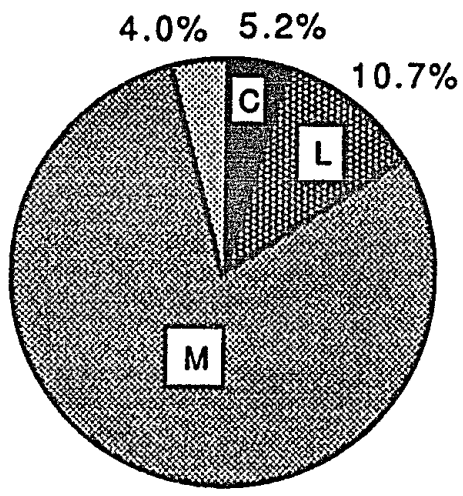

$$
\begin{aligned}
& 80.1 \% \\
& L / C=2.06
\end{aligned}
$$


TABLE 11: STATISTICS FOR SANTA CRUZ'S CBD

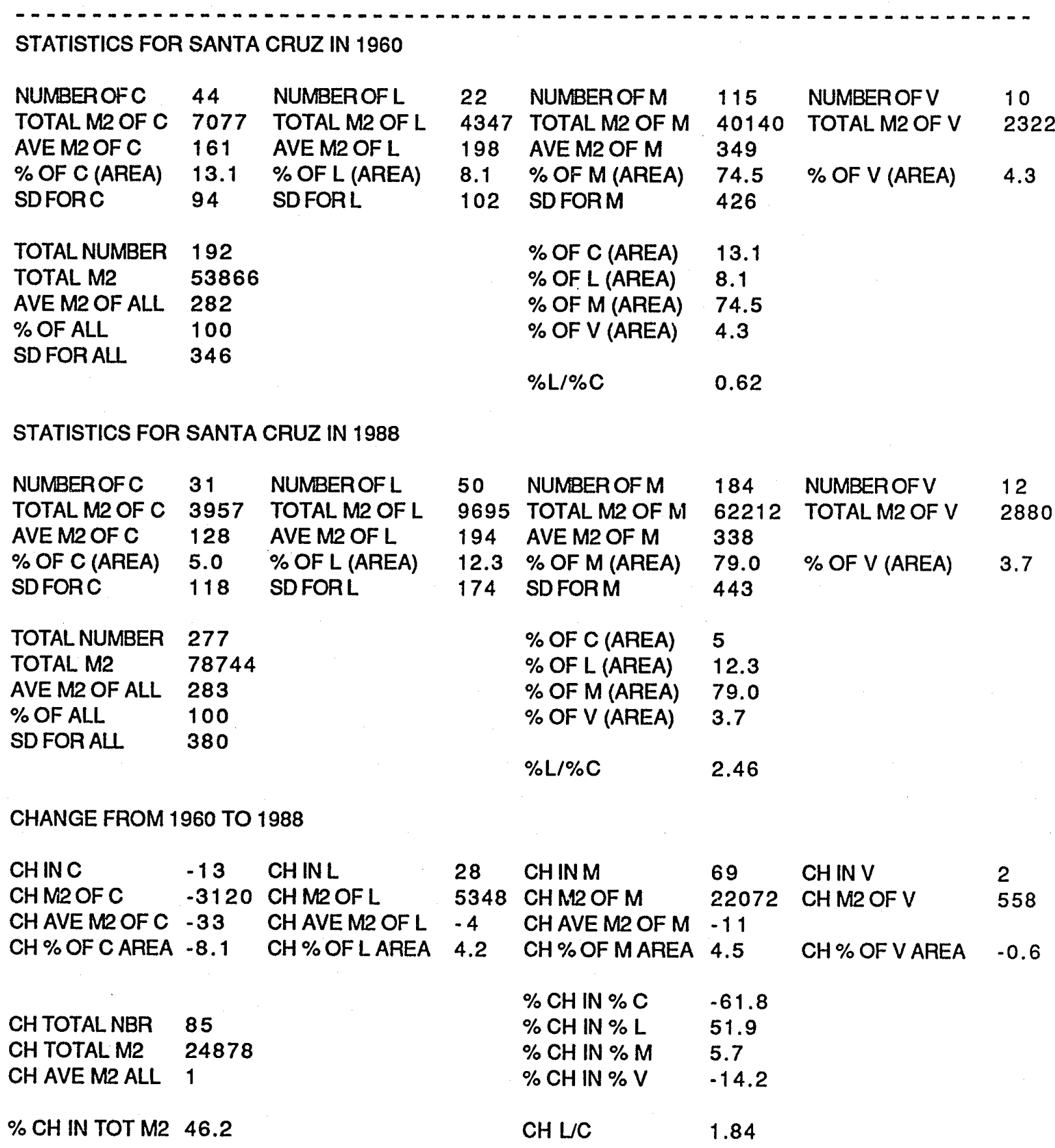


TABLE 12: STATISTICS FOR SANTA CRUZ'S MAIN STREET

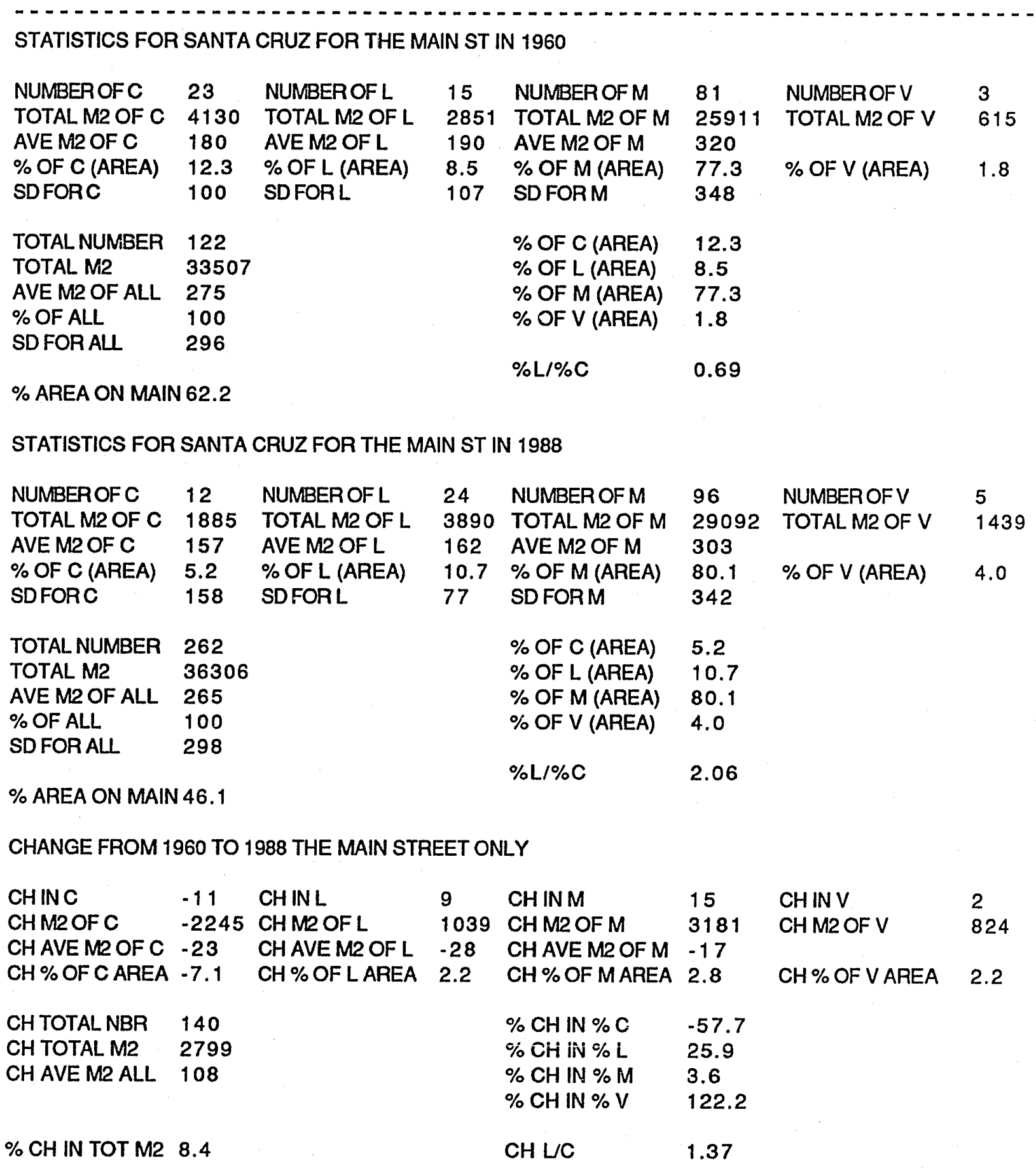




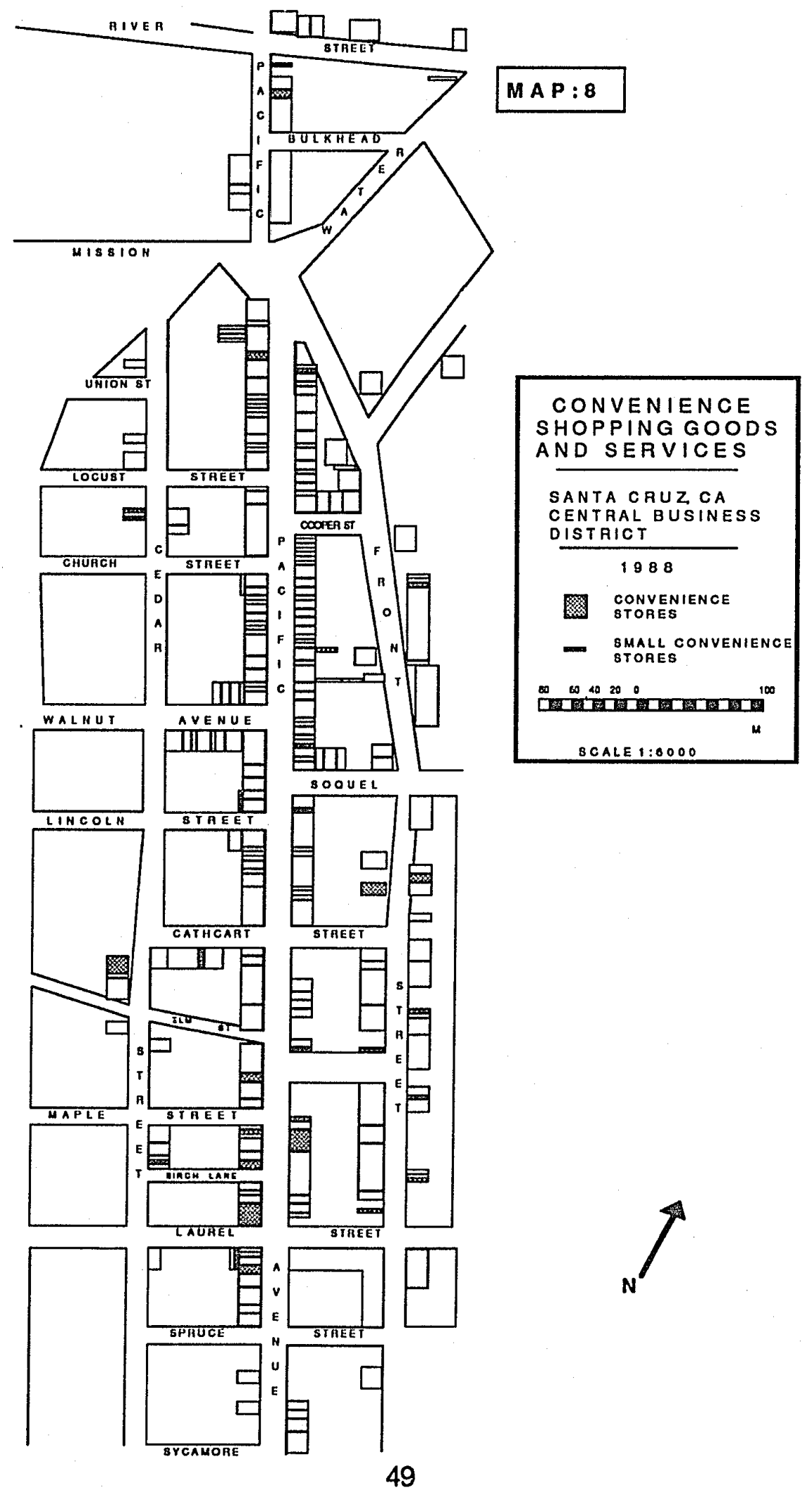


TABLE 13: CONVENIENCE STORES IN SANTA CRUZ IN 1960 BY STANDARD LAND USE CODE

Name of Establishement

MISSION DRIVE-IM MARKET

LANDESS + TOUHY MARKET

MARINOS GRO

MCHUGH AND BIANCHI GROS ***

PLAZA GROCERY

PACIFIC COOKEI CO.

MOBIL GAS ***

LAYTON'S CHEVRON GAS STATION ***

STRICKLAND'S FLYING A - gas station ***

POSTAL LUNCH REST

SADDLE ROCK- bar,coffee,barber

DELMARETTE FOUNTAIN - rest

MANHATTAN TAVERN

CLUB ALOHA TAVERN

ANCHOR CLUB TAVERN ***

MIDWAY TAVERN

ARROW CLUB TABERN-REST

THREE NINETY CLUB TAVERN

CLUB DEL MAR TAVERN

ACE CLUB TAVERN

ASTI CAFE - bar

MODEL DRUGSTORE

ECONOMY DRUGCO.

MELVIN'S DRUG STORE

FREEMAN'S BIG TREE PHARMACY

BONESIO'S LIQUOR

BUTLER'S FAMILY LIQUOR STORE

SANTA CRUZ FRENCH LAUNDRY

PAC AUTO LNDRY

SAM WO LAUNDRY

PACIFIC CLEANERS

DELUXE DRY CLEANERS AND TAILOR

CHICO'S HAIR DESIGN

FEATHEREDGE BARBER SHOP

SIM'S BEAUTY SALON

HOOVER'S BARBER SHOP

HANSEN'S IMY BARBER SHOP

POSTAL BARBER SHOP ***

PALOMAR BARBER

SIM'S BEAUTY SALON

MOBLEY'S BARBER SHOP

BEAUTY NOOK - beauty
Street Address

Standard Type Area $M^{2}$

Land Use

Code

$\begin{array}{lllll}601 & \text { FRONT STREET } & 541 & \mathrm{C} & 284 \\ 707 & \text { PACIFIC AVENUE } & 541 & \mathrm{C} & 279 \\ 601 & \text { PACIFIC AVENUE } & 541 & \mathrm{C} & 232 \\ 1553 & \text { PACIFIC AVENUE } & 541 & \mathrm{C} & 232 \\ 1534 & \text { PACIFIC AVENUE } & 541 & \mathrm{C} & 137 \\ 1214 \text { B PACIFIC AVENUE } & 546 & \mathrm{C} & 84 \\ 1102 & \text { PACIFIC AVENUE } & 553 & \mathrm{C} & 76 \\ 2001 & \text { PACIFIC AVENUE } & 553 & \mathrm{C} & 76 \\ 534 & \text { FRONT STREI } & 553 & \mathrm{C} & 76 \\ 2027 & \text { PACIFIC AVENUE } & 581 & \mathrm{C} & 163 \\ 1516 & \text { PACIFIC AVENUE } & 581 & \mathrm{C} & 112 \\ 1126 & \text { PACIFIC AVENUE } & 581 & \mathrm{C} & 56 \\ 1541 & \text { PACIFIC AVENUE } & 582 & \mathrm{C} & 358 \\ 1218 & \text { PACIFIC AVENUE } & 582 & \mathrm{C} & 205 \\ 623 & \text { PACIFIC AVENUE } & 582 & \mathrm{C} & 170 \\ 923 & \text { PACIFIC AVENUE } & 582 & \mathrm{C} & 161 \\ 1003 & \text { PACIFIC AVENUE } & 582 & \mathrm{C} & 146 \\ 711 & \text { PACIFIC AVENUE } & 582 & \mathrm{C} & 113 \\ 107 & \text { SOCUEIAVENUE } & 582 & \mathrm{C} & 109 \\ 821 & \text { PACIFIC AVENUE } & 582 & \mathrm{C} & 106 \\ 715 \mathrm{~A} & \text { PACIFIC AVENUE } & 582 & \mathrm{C} & 71 \\ 1303 & \text { PACIFIC AVENUE } & 591 & \mathrm{C} & 313 \\ 1117 & \text { PACIFIC AVENUE } & 591 & \mathrm{C} & 304 \\ 1415 & \text { PACIFIC AVENUE } & 591 & \mathrm{C} & 254 \\ 811 & \text { PACIFIC AVENUE } & 591 & \mathrm{C} & 165 \\ 801 & \text { PACIFIC AVENUE } & 592 & \mathrm{C} & 437 \\ 511 & \text { FRONT STREET } & 592 & \mathrm{C} & 337 \\ 609 & \text { PACIFIC AVENUE } & 621 & \mathrm{C} & 225 \\ 690 & \text { PACIFIC AVENUE } & 621 & \mathrm{C} & 139 \\ 823 & \text { PACIFIC AVENUE } & 621 & \mathrm{C} & 106 \\ 825 & \text { PACIFIC AVENUE } & 621 & \mathrm{C} & 106 \\ 719 & \text { PACIFIC AVENUE } & 621 & \mathrm{C} & 60 \\ 1521 & \text { PACIFIC AVENUE } & 623 & \mathrm{C} & 250 \\ 905 & \text { PACIFIC AVENUE } & 623 & \mathrm{C} & 160 \\ 1514 & \text { PACIFIC AVENUE } & 623 & \mathrm{C} & 160 \\ 1009 & \text { PACIFIC AVENUE } & 623 & \mathrm{C} & 146 \\ 102 & \text { WALNUT AVENUE } & 623 & \mathrm{C} & 106 \\ 2025 & \text { PACIFIC AVENUE } & 623 & \mathrm{C} & 96 \\ 1332 & \text { PALOMAR ARC } & 623 & \mathrm{C} & 96 \\ 1333 & \text { PALOMAR ARC } & 623 & \mathrm{C} & 96 \\ 115 & \text { WALNUT AVENUE } & 623 & \mathrm{C} & 90 \\ 1122 & \text { PACIFIC AVENUE } & 623 & \mathrm{C} & 56 \\ 50 & & & & \\ & & & \end{array}$


SAM'S SHOE HOSPITAL REPAIR

FINAGAL WATCH REPAIR
227

CATHCART ST

1332 PALOMAR ARC

44

7077

161

13.1

94

*** Establishment assigned approximate value for area. 


\section{Limited Appeal Space}

Since only slightly over eight percent of the total retail space was used by limited appeal stores in 1960, many blocks had no limited appeal stores. A small clustering of limited appeal stores appears on the south side of Walnut Avenue. (see map 9) The limited appeal stores include a variety of types of stores, but few could be called luxury item stores. There were a couple of pet stores, church supply stores, and music stores. (see table 14)

Limited appeal space grew from $8.1 \%$ to $12.3 \%$ of the total retail space. The concurrent drop in convenience stores increased the ratio of limited appeal space to convenience space from .62 to 2.46. Limited appeal stores now take up almost two and a half times more space than convenience stores do. The type of limited appeal goods has also changed over the years. There are now several luxury type stores, including two antique shops and two art galleries. Drug paraphanalia, New Age books, and crystal stores reveal Santa Cruz's liberalism. (see table 15) The limited appeal stores are distributed throughout the downtown. (see map 10) Many of them are in the "mall" area of Pacific Avenue. There is a small clustering of limited appeal stores on River Street near Pacific Avenue. Although the cluster of limited appeal stores that was on Walnut Avenue in 1960 still has three such stores in it, it no longer stands out from the rest of the CBD. The few limited appeal stores that have moved onto Front Street are non-luxury establishments. They include a camera shop, a store for recycled stereos, and a specialty auto repair garage. 
TABLE 14 : CONVENIENCE STORES IN SANTA CRUZ IN 1988 BY STANDARD LAND USE CODE

\begin{tabular}{|c|c|c|c|c|c|}
\hline Name of Establishement & Stree & Address & $\begin{array}{l}\text { Standar } \\
\text { Land Us } \\
\text { Code }\end{array}$ & Ty & Area $\mathrm{M}^{2}$ \\
\hline NATURES BOUNTY - grocery & 811 & PACIFIC AVENUE & 541 & C & 165 \\
\hline PACIFIC COOKEI CO. & 1215 & PACIFIC AVENUE & 546 & C & 84 \\
\hline PACIFIC COOKIE CO. 2 & 920 & PACIFIC AVENUE & 546 & C & 35 \\
\hline SHELL - gas & 300 & FRONT STREET & 553 & C & 20 \\
\hline DOUBLE RAINBOW - Icecream & 110 & COOPERSTREET & 581 & C & 75 \\
\hline DELMARETTE FOUNTAIN & 1126 & PACIFIC AVENUE & 581 & C & 56 \\
\hline BURRITO EXPRESS & 920 & PACIFIC AVENUE & 581 & $\mathrm{C}$ & 30 \\
\hline HAMBURGERSHOPPE & 314 & FRONT STREET & 581 & C & 23 \\
\hline TAMPICO LOUNGE - bar & 820 & PACIFIC AVENUE & 582 & C & 512 \\
\hline FRONT STREET PUB - bar & 516 & FRONT STREET & 582 & C & 296 \\
\hline LULU CARPENTER'S - bar & 1545 & PACIFIC AVENUE & 582 & C & 165 \\
\hline BLUE LAGOON - bar & 923 & PACIFIC AVENUE & 582 & C & 161 \\
\hline SILVER BULLET - bar & 603 & FRONT STREET & 582 & C & 126 \\
\hline AVENUE, THE - bar & 711 & PACIFIC AVENUE & 582 & C & 113 \\
\hline POET + PATRIOT IRISH PUB & 320 & CEDAR STREET & 582 & C & 75 \\
\hline ASTI CAFE - bar & 716 & PACIFIC AVENUE & 582 & C & 71 \\
\hline ECONONY DRUGSTOFE & 907 & CEDAR STREET & 591 & C & 75 \\
\hline LUQORSTORE & 801 & PACIFIC AVENUE & 592 & C & 437 \\
\hline WAREHOUSE LQUUORS & 511 & FRONT STREET & 592 & C & 337 \\
\hline PACIFIC DRY CLEANER & 511 & CEDARSTREET & 621 & C & 183 \\
\hline ROYAL CLEANERS & 108 & LINCOLNST & 621 & C & 91 \\
\hline JOE'S BARBER SHOP & 824 & PACIFIC AVENUE & 623 & C & 116 \\
\hline PERFECT CUT & 823 & PACIFIC AVENUE & 623 & c & 106 \\
\hline CANET LTD - beauty & 913 & CEDAR STREET & 623 & C & 77 \\
\hline HAIR STYLES ETC & 2044 & PACIFIC AVENUE & 623 & C & 63 \\
\hline MINA'S - beauty & 716 & FRONT STREET & 623 & C & 38 \\
\hline MALL BARBER SHOP & 1540 & PACIFIC AVENUE & 623 & C & 18 \\
\hline JACK'S SHOE SERVICE & 227 & CATHCART ST & 625 & C & 83 \\
\hline KINKO'S COPIES & 412 & FRONT STREET & 633 & C & 161 \\
\hline UNIVERSITY COPY & 429 & FRONT STREET & 633 & C & 119 \\
\hline FINGAL WATCH REPAIR & 6 & PALOMAR ARC. & 649 & C & 46 \\
\hline NUMBEROF C & & 31 & & & \\
\hline TOTAL M2 OF C & & 3957 & & & \\
\hline AVE M2 OF C & & 128 & & & \\
\hline$\%$ OF C (AREA) & & 5.0 & & & \\
\hline SDFORC & & 118 & & & \\
\hline
\end{tabular}




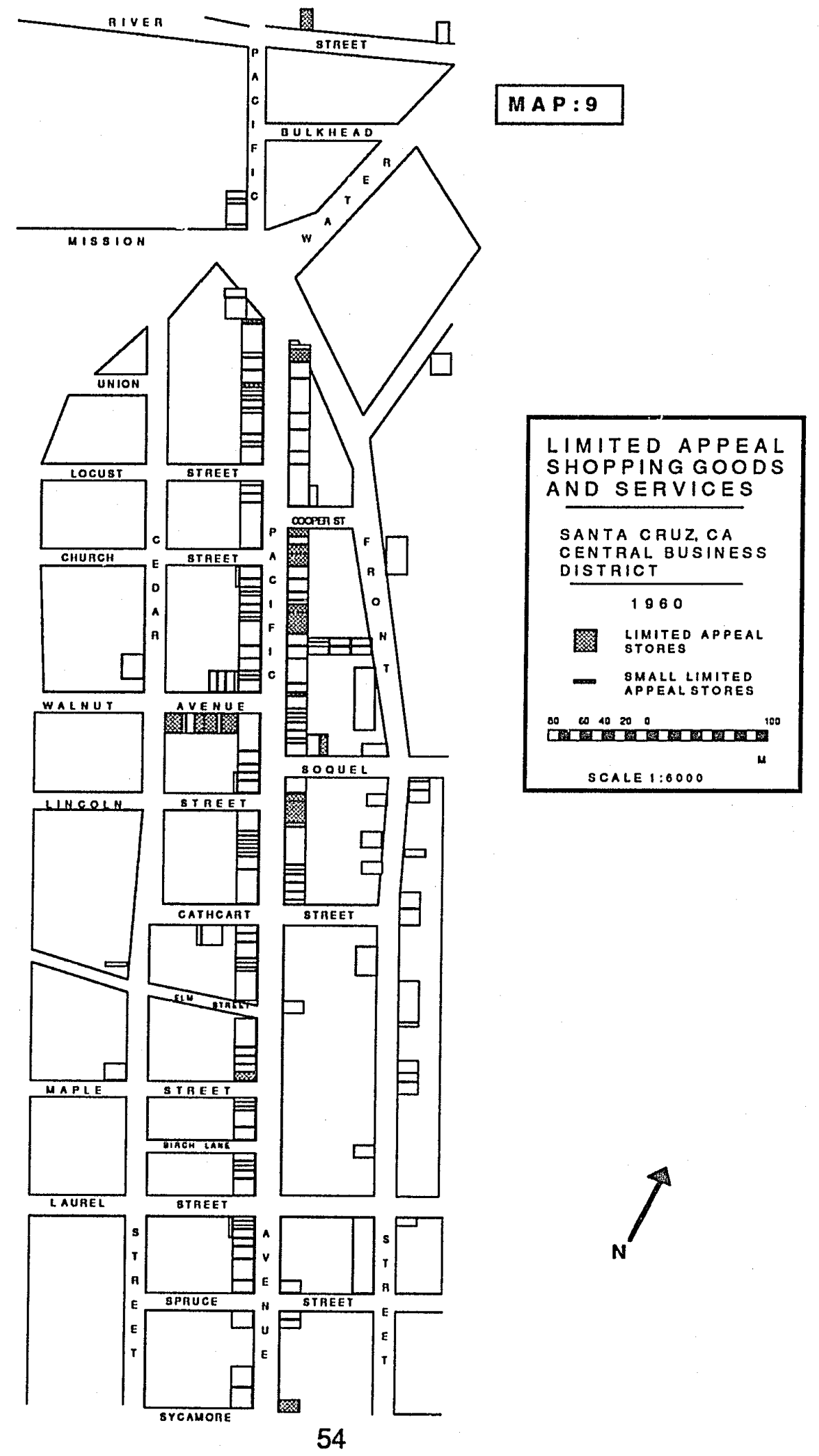


TABLE 15: LIMITED APPEAL STORES IN SANTA CRUZ IN 1960 BY STANDARD LAND USE CODE

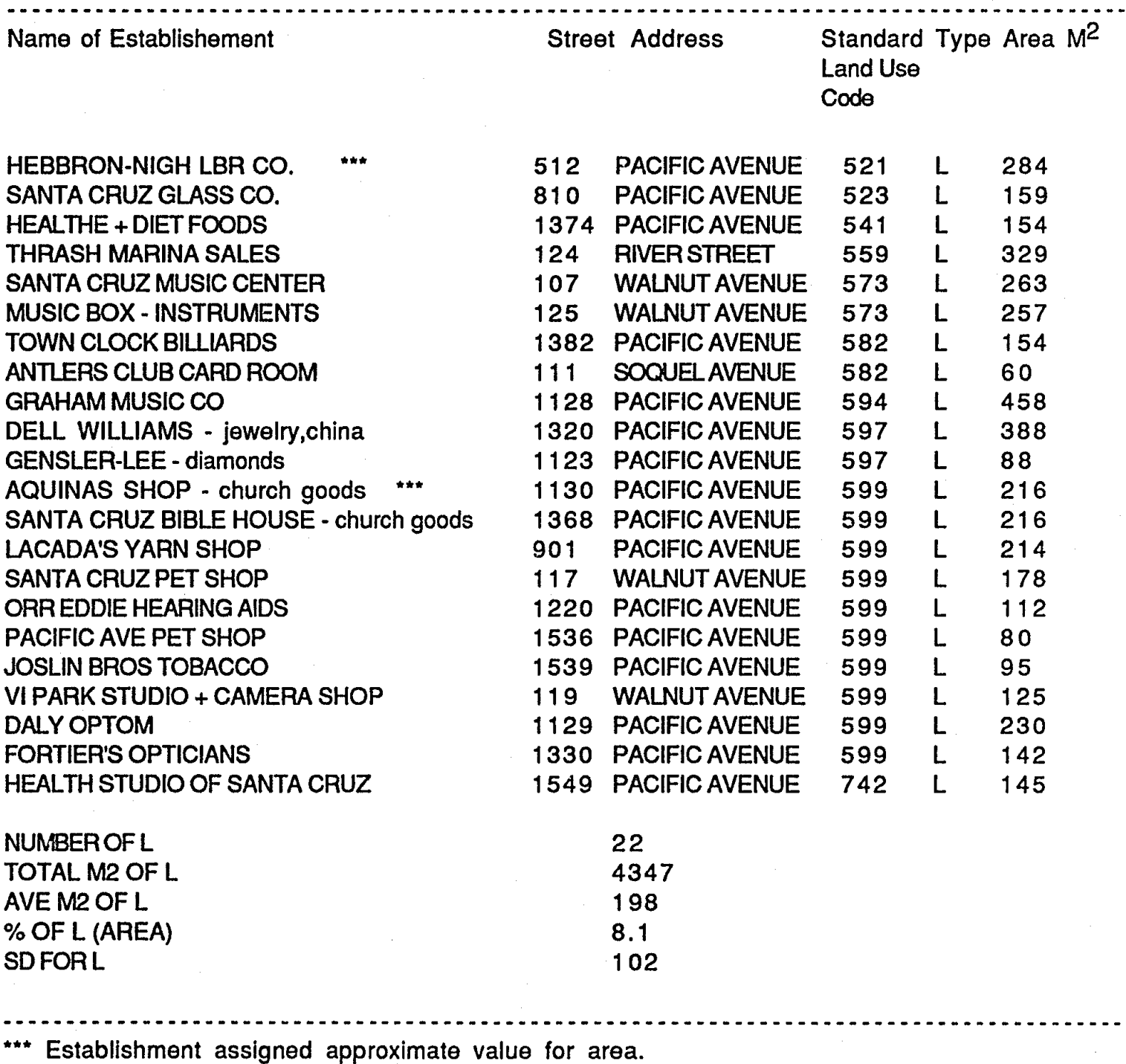




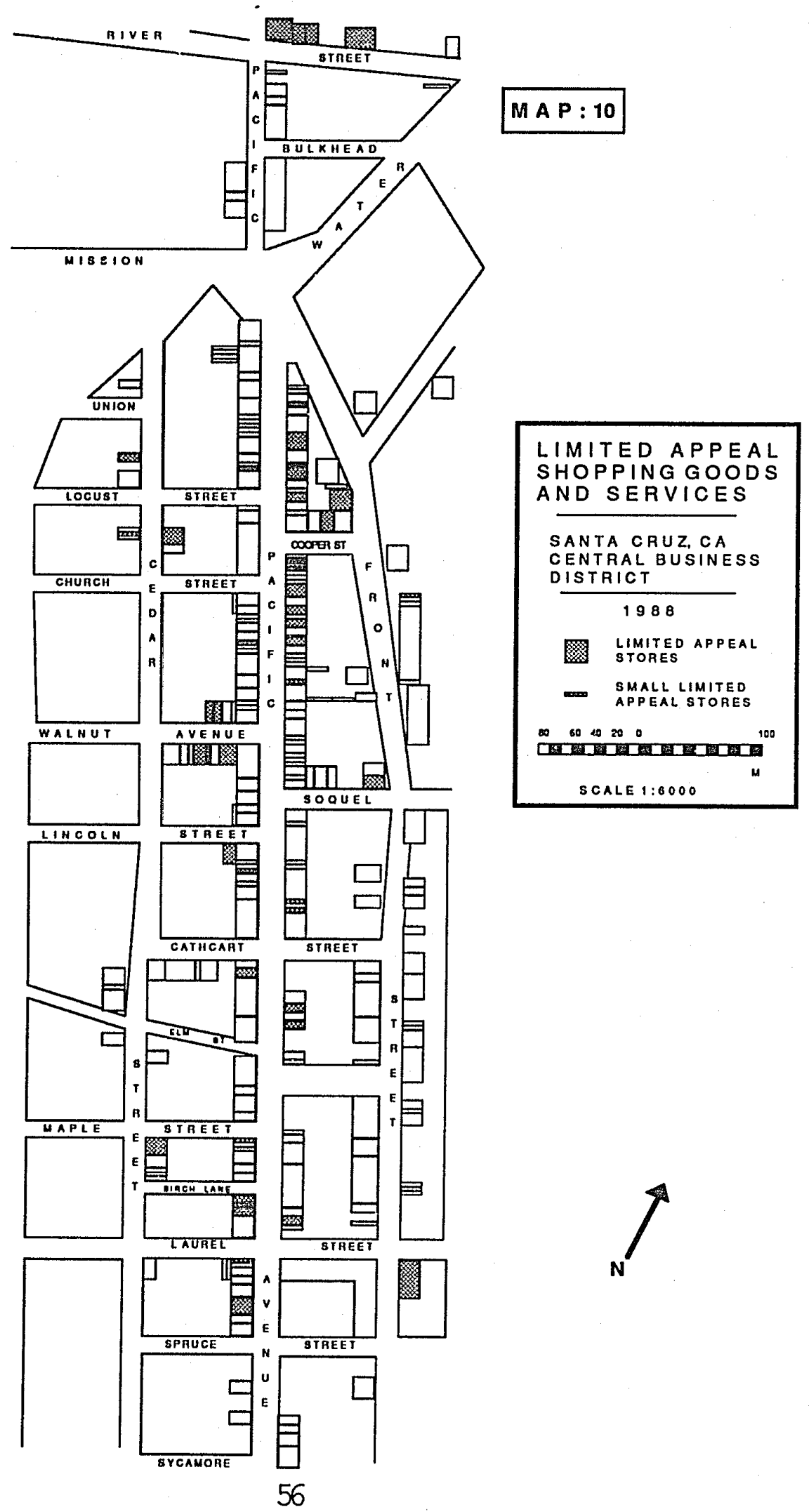




\section{Mass Appeal Space}

In $1960,74.5 \%$ of all the retail floorspace was used by mass appeal stores. This included a couple of very large department stores on Pacific Avenue, (see map 11) which together accounted for almost ten percent of the total mass appeal space. There was also a large car dealership and a large furniture store. (see table 16) These large stores brought the average size of a mass appeal store to $349 \mathrm{~m}^{2}$ of floorspace.

Mass appeal grew from $74.5 \%$ to $79.0 \%$ of the total retail space. Bank space more than doubled from $4.2 \%$ to $9.1 \%$. As one would expect from the growing student population, stationary and record stores grew from $2.7 \%$ of the total retail space to $7.0 \%$. Restaurants grew from $6.2 \%$ to $16.1 \%$. Clothing stores remained at about $15 \%$. Real Estate offices, however, decreased from $3.0 \%$ to only .3\% of the total mass appeal space. (see table 17; map 12)

\section{The Main street}

Pacific Avenue, from Laurel Street to Mission Street, is considered to be Santa Cruz's "main street." This includes the addresses from 800 to 1599 Pacific Avenue. Just over $62 \%$ of the total retail space was located on the "main street" in 1960. The size of stores on average for the main street was slightly smaller for each type $(C, L, M)$, as well as for all types combined.

The "main street" of Santa Cruz has been made into a "mall" area. 


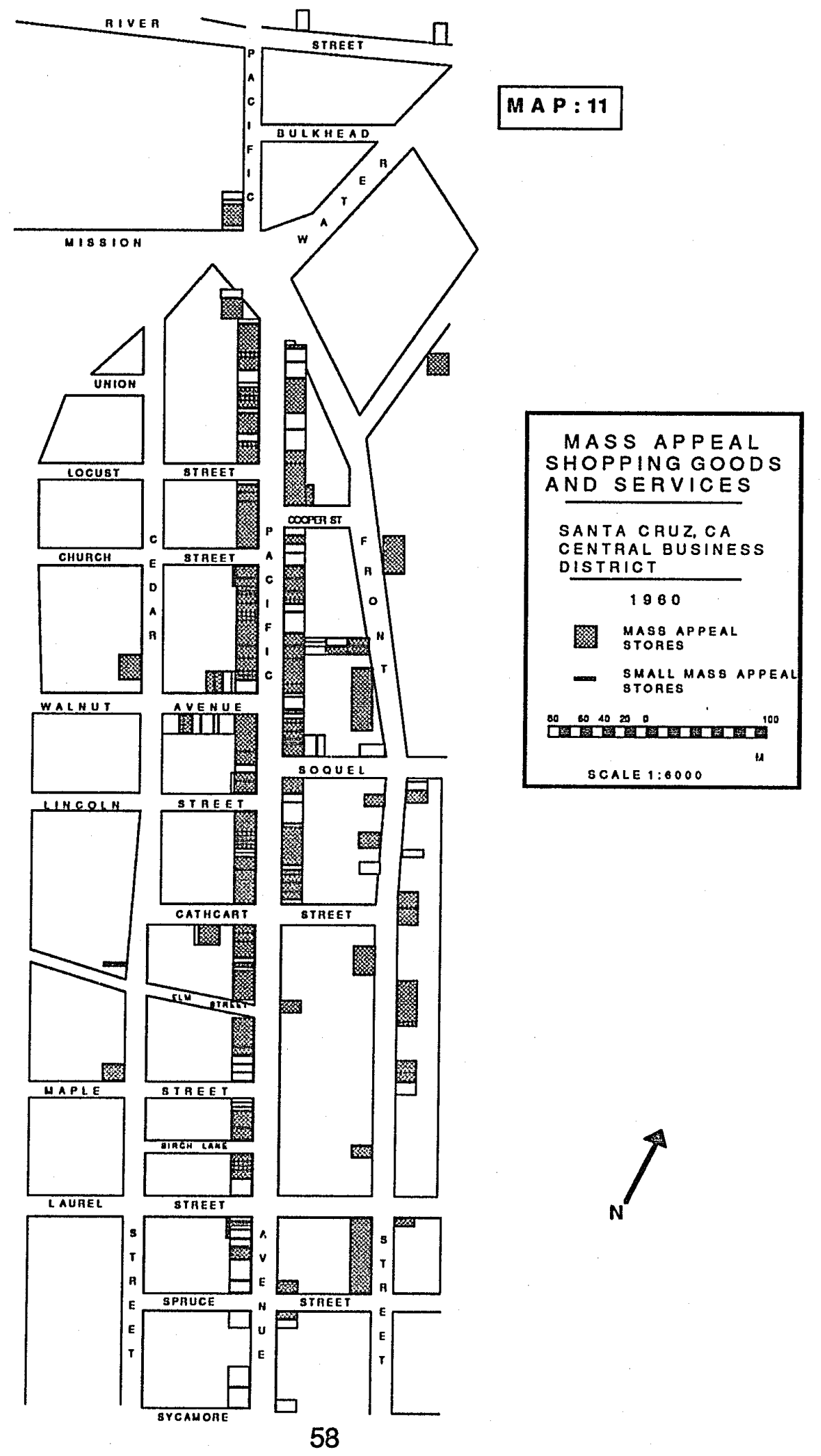


TABLE 16: LIMITED APPEAL STORES IN SANTA CRUZ IN 1988 BY STANDARD LAND USE CODE

\begin{tabular}{|c|c|c|c|c|c|}
\hline Name of Establishement & Stree & t Address & $\begin{array}{l}\text { Stand } \\
\text { Land L } \\
\text { Codo }\end{array}$ & & \\
\hline SANTA CRUZ GLASS CO. & 806 & PACIFIC AVENUE & 523 & L & \\
\hline SANTA CRUZ NUTRITION CENTER & 1374 & PACIFIC AVENUE & 541 & $\mathrm{~L}$ & \\
\hline WHOLE ANIMAL SOURCE STORE - pet food & 911. & CEDAR STREET & 549 & L & 5 \\
\hline CYCLEIMAGERY - motorcycle things & 401 & FRONT STREET & 559 & $\mathbf{L}$ & 94 \\
\hline BRIT-KNIT CO. & 110 & COOPERSTREET & 563 & L & 5 \\
\hline CAMOUFLAGE - clothes & 1212 & PACIFIC AVENUE & 565 & $\mathbf{L}$ & 205 \\
\hline MODERNE LIFE - costumes & 1531 & PACIFIC AVENUE & 569 & L & \\
\hline DREAMSWEET - futons & 1528 & PACIFIC AVENUE & 571 & L & \\
\hline ACT FIVE - futons & 110 & COOPERSTREET & 571 & L & \\
\hline RECYCLED STEREOPLUS & 811 & FRONT STREET & 573 & L & 30 \\
\hline ECLECTIX - used goods, antiques & 803 & PACIFIC AVENUE & 593 & L & 36 \\
\hline LANTIQUE COLLECTIVE & 1526 & IVENUE & 593 & L & \\
\hline UNION GROVE MUSIC & 1013 & PACIFIC AVENUE & 594 & $L$ & \\
\hline FOURWINDSMUSIC & 320 & CEDAR STREET & 594 & $\mathbf{L}$ & \\
\hline WESTERN UNION - cards & 920 & PACIFIC AVENUE & 594 & L & \\
\hline SKI SHOP & 124 & STREET & 595 & L & \\
\hline OUTDOORWORLD & 136 & RIVER STREET & 595 & L & \\
\hline LENZ & 142 & STREET & 599 & L & 84 \\
\hline CANTORIWHEAT GALLERY - art & 107 & WALNUT AVENUE & 599 & L & 263 \\
\hline ARTISANS & 1364 & PACIFIC AVENUE & 599 & $\mathbf{L}$ & 216 \\
\hline ING CO. & 1514 & PACIFIC AVENUE & 599 & $\mathbf{L}$ & 16 \\
\hline GALLER & 111 & STREET & 599 & L & 15 \\
\hline ROCK ST & 1007 & CEDAR STREET & 599 & L & 29 \\
\hline IVEL ACCESSORIES & 1349 & AVENUE & 599 & L & 22 \\
\hline DSMETICS & 108 & VENUE & 599 & L & 8 \\
\hline GRAPHFIX - art & 1517 & PACIFIC AVENUE & 599 & $L$ & 1 \\
\hline PIPE-LINE -drug para. & 1121 & PACIFIC AVENUE & 599 & L & \\
\hline ATHERS, FINS & 1536 & AVENUE & 599 & L & 80 \\
\hline BEAD IT & 805 & AVENUE & 599 & $L$ & 29 \\
\hline WEBBER'S & 1396 & PACIFIC AVENUE & 599 & L & 326 \\
\hline CAMERA W & 710 & FRONT STREET & 599 & L & 43 \\
\hline ITASY WORLD & 707 & VENUE & 599 & L & 279 \\
\hline PACIFIC GARDEN IMPORTS & 1110 & PACIFIC AVENUE & 599 & L & 138 \\
\hline FOLK ARTS & 1110 & PACIFIC AVENUE & 599 & L & $13 \varepsilon$ \\
\hline TOOLSFORFOOLS & 516 & CEDARSTREET & 599 & $\mathbf{L}$ & $12 \varepsilon$ \\
\hline GIFTS AND ART GALLERY & 110 & WALNUT AVENUE & 599 & L & 118 \\
\hline GATEWAYS - new age books + gifts & 825 & PACIFIC AVENUE & 599 & $\mathbf{L}$ & 106 \\
\hline GREENPE & 1012 & PACIFIC AVENUE & 599 & L & 91 \\
\hline CRYSTAL KALEIDOSCOPE & 110 & COOPERSTREET & 599 & L & 35 \\
\hline SUN SHADE OPTIQUE & 1382 & PACIFIC AVENUE & 599 & L & 154 \\
\hline FORTIER'S OPTICIANS & 1330 & PACIFIC AVENUE & 599 & L & 142 \\
\hline PACIFIC GOLD+ SILVERE & 719 & PACIFIC AVENUE & 613 & L & 6 \\
\hline PIP PRINTING & 809 & PACIFIC AVENUE & 633 & L & \\
\hline
\end{tabular}




SENTINEL PRINTERS
BUGFORMANCE - VW parts + service
HENNINGS FOREIGN AUTO REPAIR
LEN ROACH - mercedes repair
EXECUTIVE DETAIL SHOP
SHAYER SHOP - sharpening
START - health club
NUMBER OFL
TOTAL M2 OF L
AVE M2 OFL
\% OF L (AREA)
SD FORL

$\begin{array}{lllll}912 & \text { CEDARSTREET } & 633 & \text { L } & 174 \\ 222 & \text { FRONT STREET } & 641 & \text { L } & 1012 \\ 116 & \text { RIVERSTREET } & 641 & \text { L } & 645 \\ 512 & \text { PACIFIC AVENUE } & 641 & \text { L } & 99 \\ 512 & \text { PACIFIC AVENUE } & 641 & \text { L } & 99 \\ 115 & \text { WALNUT AVENUE } & 699 & \text { L } & 90 \\ 113 & \text { LINCOLN ST } & 742 & \text { L } & 339 \\ & & & & \\ & 50 & & & \\ & 9695 & & & \\ 194 & & & \\ 12.3 & & & \\ 174 & & & & \end{array}$


TABLE 17: MASS APPEAL STORES IN SANTA CRUZ IN 1960 BY STANDARD LAND USE CODE

\begin{tabular}{|c|c|c|c|c|c|}
\hline Name of Establishement & Stree & Address & $\begin{array}{l}\text { Standar } \\
\text { Land Us } \\
\text { Code }\end{array}$ & & \\
\hline RIORDAN-WINETT TRAVEL AGCY & 1376 & PACIFIC AVENUE & 492 & M & 154 \\
\hline FARRAR-HALBACH BUIK - used car sales & 702 & PACIFIC AVENUE & 512 & M & 284 \\
\hline CIMARELLI'S PLUMBING CO. & 209 & LAUREL STREET & 522 & M & 63 \\
\hline QUALITY PAINT STORE & 939 & PACIFIC AVENUE & 523 & M & 659 \\
\hline SANTA CRUZ HARDWARE & 1523 & PACIFIC AVENUE & 525 & M & 580 \\
\hline BYREN BROS HARDWARE & 1531 & PACIFIC AVENUE & 525 & M & 314 \\
\hline JOHNSON'S HARDWARE & 1214 & PACIFIC AVENUE & 525 & $M$ & 168 \\
\hline LEASK'S DEPT STORE & 1405 & PACIFIC AVENUE & 531 & M & 2235 \\
\hline JC PENNY & 1101 & PACIFIC AVENUE & 531 & M & 1736 \\
\hline NATIONAL DOLLAR STORES - dept store & 1111 & PACIFIC AVENUE & 531 & M & 726 \\
\hline WOOLWORTHS & 1213 & PACIFIC AVENUE & 531 & M & 706 \\
\hline SEARS & 1112 & PACIFIC AVENUE & 531 & M & 230 \\
\hline MONTGOMERY WARD & 1510 & PACIFIC AVENUE & 531 & $M$ & 92 \\
\hline VAN'S SUPER MARKET & 611 & FRONT STREET & 541 & $\mathbf{M}$ & 1411 \\
\hline PURE FOOD CENTER - grosery & 1547 & PACIFIC AVENUE & 541 & $M$ & 855 \\
\hline MACFARLANE'S CANDIES & 1363 & PACIFIC AVENUE & 544 & $M$ & 172 \\
\hline BUBBLE BAKERY & 1541 & PACIFIC AVENUE & 546 & M & 358 \\
\hline SPECIALTY FOOD SHOP - bakery & 419 & CEDAR STREET & 546 & $M$ & 249 \\
\hline PLAZA BAKERY & 1540 & PACIFIC AVENUE & 546 & M & 43 \\
\hline FARRAR-HALBACH BUICKCCONTINENTAL & 201 & FRONT STREET & 551 & M & 2896 \\
\hline CENTRAL ONE STOP AUTO DEALER *** & 714 & FRONT STREET & 551 & $\mathbf{M}$ & 600 \\
\hline MAYCRINK PONTIAC-CADILLAC DEALERS & 429 & FRONT STREET & 551 & $M$ & 582 \\
\hline ALDERSON AUTO DEALERS & 521 & FRONT STREET & 551 & $\mathbf{M}$ & 503 \\
\hline HOLAND AUTO DEALERS & 325 & FRONT STREET & 551 & M & 435 \\
\hline PROLO CHEVROLET CO, - auto dealers & 920 & PACIFIC AVENUE & 551 & M & 91 \\
\hline SWEET SERV CO. - auto parts & 504 & FRONT STREET & 552 & M & 648 \\
\hline TIRE SERV CENTER & 525 & FRONT STREET & 552 & M & 374 \\
\hline BIBBINS TIRE SERV & 2015 & PACIFIC AVENUE & 552 & $\mathbf{M}$ & 374 \\
\hline KELLEY'S SERV INC - auto parts & 516 & FRONT STREET & 552 & $\mathbf{M}$ & 296 \\
\hline MACHINE SHOP SERVAUTO PARTS & 703 & FRONT STREET & 552 & M & 265 \\
\hline ABRAMS MORRIS STORE - men's clothes & 1129 & PACIFIC AVENUE & 561 & M & 925 \\
\hline HUB,THE - men's clothes & 1118 & PACIFIC AVENUE & 561 & M & 230 \\
\hline HARRIS BROS - men's clothes & 1212 & PACIFIC AVENUE & 561 & M & 205 \\
\hline LEIBBRAHDT + PUGET - men's clothes & 1216 & PACIFIC AVENUE & 561 & M & 112 \\
\hline RALSTON'S APPAREL - women's clothes & 1329 & PACIFIC AVENUE & 562 & $\mathbf{M}$ & 248 \\
\hline MCCALL'S MAPLE SHOP & 803 & PACIFIC AVENUE & 562 & M & 236 \\
\hline JAY VEE SHOP - women's clothes & 1308 & PACIFIC AVENUE & 562 & M & 233 \\
\hline MODE O'DAY - clothes & 1379 & PACIFIC AVENUE & 562 & M & 150 \\
\hline SWINK DORA DRESS SHOP & 1367 & PACIFIC AVENUE & 562 & $M$ & 132 \\
\hline TREND O' FASHION - women's & 1315 & PACIFIC AVENUE & 562 & M & 129 \\
\hline LAZETTE FEMININE APPAREL & 1345 & PACIFIC AVENUE & 562 & M & 122 \\
\hline RITTENHOUSE LADIES APPAREL & 1373 & PACIFIC AVENUE & 562 & M & 113 \\
\hline
\end{tabular}


WOMAN'S SHOP

CASUAL AIRE - women's clothes

ADVENTURE IN TOYS

MOHONDRO + SPECIALTY SHOP - child.'s

SANTA CRUZ SPORTSWEAR CO

SCHIPPER-DILLON - clothes

VETERANS SALVAGE SHOP - used clothes

LAD-N-LASSIE SHOP

SANTA CRUZDISCOUNT HOUSE

REEVES SHOES

GALLEN KAMP'S SHOES

KARL'S SHOE STORE

SUTTON'S OF SANTA CRUZ - shDOS

NEW MODEL SHOE SHOP

HABER'S - furniture

GENERAL FURNCO.

MCCALIS FURN $C O$.

HOME DECORATNE SHOP

MERCANTILE ACCEPTANCE CO.

SINGER SEWING CENTER

SPARKY'S TV SALES AND SERVICE

ORCHARD'SREST

HILDA'S REST

CAFE CAMELEON

MOCK'S DRUGS-REST

DICK'S FINE FOOD - rest

COAST CAEAMERY REST

ANVENUE GRILL REST ***

TAMPICO - rest

NEW CHINA CAFE - REST

PALOMAR GRILL

HABER'S THRIFT STORE FURNITURE

FIELDS GENL MDSE USED

DON'S PLAZA STATIONERY STORE

PALACE STATIONERS

BOWMAN-FORGEY STATIONARY

UNIVERSE RECORDS + TAPES

PAC BOOK SHOPPE

JOHNNY'S BIKE AND SPORT SHOP

SPORTSMAN'S SHOP

STEEN'S CREDIT JWLRS

PLAZA JWLRY RET

SHOCKLEY'S JWLRS

PENNEL'S JEWELERS

FRABONI JWLRS

ZELL CREDIT JEWELERS

ALEXANDER'S JWLRS

FERRARI THE FLORIST

PALOMAR HOUSE OF FLOWERS ***

$\begin{array}{lllll}1535 & \text { PACIFIC AVENUE } & 562 & \mathrm{M} & 100 \\ 1121 & \text { PACIFIC AVENUE } & 562 & \mathrm{M} & 88 \\ 1113 & \text { PACIFIC AVENUE } & 564 & \mathrm{M} & 242 \\ 1360 & \text { PACIFIC AVENUE } & 564 & \mathrm{M} & 216 \\ 440 & \text { FRONT STREET } & 565 & \mathrm{M} & 758 \\ 1224 & \text { PACIFIC AVENUE } & 565 & \mathrm{M} & 434 \\ 809 & \text { PACIFIC AVENUE } & 565 & \mathrm{M} & 254 \\ 1306 & \text { PACIFIC AVENUE } & 565 & \mathrm{M} & 209 \\ 709 & \text { PACIFIC AVENUE } & 565 & \mathrm{M} & 205 \\ 1209 & \text { PACIFIC AVENUE } & 566 & \mathrm{M} & 285 \\ 1201 & \text { PACIFIC AVENUE } & 566 & \mathrm{M} & 242 \\ 1125 & \text { PACIFIC AVENUE } & 566 & \mathrm{M} & 187 \\ 1349 & \text { PACIFIC AVENUE } & 566 & \mathrm{M} & 122 \\ 1537 & \text { PACIFIC AVENUE } & 566 & \mathrm{M} & 70 \\ 812 & \text { PACIFIC AVENUE } & 571 & \mathrm{M} & 992 \\ 1001 & \text { PACIFIC AVENUE } & 571 & \mathrm{M} & 800 \\ 1010 & \text { PACIFIC AVENUE } & 571 & \mathrm{M} & 146 \\ 121 & \text { WALNUT AVENUE } & 571 & \mathrm{M} & 143 \\ 701 & \text { FRONT STREET } & 572 & \mathrm{M} & 622 \\ 1325 & \text { PACIFIC AVENUE } & 572 & \mathrm{M} & 248 \\ 1013 & \text { PACIFIC AVENUE } & 573 & \mathrm{M} & 233 \\ 1335 & \text { PACIFIC AVENUE } & 581 & \mathrm{M} & 573 \\ 221 & \text { CATHCART ST } & 581 & \mathrm{M} & 310 \\ 418 & \text { FRONT STREET } & 581 & \mathrm{M} & 306 \\ 1387 & \text { PACIFIC AVENUE } & 581 & \mathrm{M} & 247 \\ 1116 & \text { PACIFIC AVENUE } & 581 & \mathrm{M} & 230 \\ 806 & \text { PACIFIC AVENUE } & 581 & \mathrm{M} & 201 \\ 698 & \text { PACIFIC AVENUE } & 581 & \mathrm{M} & 194 \\ 822 & \text { PACIFIC AVENUE } & 581 & \mathrm{M} & 149 \\ 1007 & \text { PACIFIC AVENUE } & 581 & \mathrm{M} & 146 \\ 1352 & \text { PACIFIC AVENUE } & 581 & \mathrm{M} & 146 \\ 428 & \text { FRONT STREET } & 593 & \mathrm{M} & 1689 \\ 813 & \text { PACIFIC AVENUE } & 593 & \mathrm{M} & 165 \\ 1551 & \text { PACIFIC AVENUE } & 594 & \mathrm{M} & 620 \\ 1364 & \text { PACIFIC AVENUE } & 594 & \mathrm{M} & 216 \\ 1319 & \text { PACIFIC AVENUE } & 594 & \mathrm{M} & 123 \\ 1214 \text { APACIFIC AVENUE } & 594 & \mathrm{M} & 84 \\ 717 & \text { PACIFIC AVENUE } & 594 & \mathrm{M} & 60 \\ 1017 & \text { PACIFIC AVENUE } & 595 & \mathrm{M} & 304 \\ 1532 & \text { PACIFIC AVENUE } & 595 & \mathrm{M} & 242 \\ 1411 & \text { PACIFIC AVENUE } & 597 & \mathrm{M} & 468 \\ 1208 & \text { PACIFIC AVENUE } & 597 & \mathrm{M} & 207 \\ 1205 & \text { PACIFIC AVENUE } & 597 & \mathrm{M} & 177 \\ 1359 & \text { PACIFIC AVENUE } & 597 & \mathrm{M} & 172 \\ 927 & \text { PACIFIC AVENUE } & 597 & \mathrm{M} & 161 \\ 1519 & \text { PACIFIC AVENUE } & 597 & \mathrm{M} & 101 \\ 1533 & \text { PACIFIC AVENUE } & 597 & \mathrm{M} & 100 \\ 1222 & \text { PACIFIC AVENUE } & 599 & \mathrm{M} & 150 \\ 1332 & \text { PALOMAR ARC } & 599 & \mathrm{M} & 118 \\ 62 & & & \\ & & & \end{array}$




\begin{tabular}{|c|c|c|c|c|c|}
\hline FARMERS AND MERCHANTS NATIONAL BANK & 1515 & PACIFIC AVENUE & 611 & $M$ & 466 \\
\hline SANTA CRUZ SAVINGS AND LOAN & 1407 & PACIFIC AVENUE & 611 & M & 400 \\
\hline BANK OF AMERICA & 1134 & PACIFIC AVENUE & 611 & M & 325 \\
\hline COUNTY BANK OF SANTA CRUZ & 1502 & PACIFIC AVENUE & 611 & M & 304 \\
\hline COUNTY FIRST NATIONAL BANK & 107 & COOPERSTREET & 611 & $\mathbf{M}$ & 179 \\
\hline DARROW REALTY & 817 & PACIFIC AVENUE & 615 & M & 279 \\
\hline HALL REAL ESTATE & 1545 & PACIFIC AVENUE & 615 & M & 165 \\
\hline BLAKE REALTOR & 110 & WALNUT AVENUE & 615 & M & 118 \\
\hline BLAKE, C H REALTOR & 110 & WALNUT AVENUE & 615 & $M$ & 118 \\
\hline KOON REAL ESTATE & 1348 & PACIFIC AVENUE & 615 & M & 106 \\
\hline RHODES AND VELLUTINE REAL ESTATE *** & 128 & LINCOLN STREET & 615 & M & 92 \\
\hline PATTERSON REAL ESTATE & 123 & WALNUT AVENUE & 615 & M & 71 \\
\hline FRUNUES + FALLIS REAL ESTATE & 1210 & PACIFIC AVENUE & 615 & M & 69 \\
\hline RYSDAM REAL ESTATE & 1120 & PACIFIC AVENUE & 615 & M & 56 \\
\hline KUXELL REAL ESTATE & 107 & CHURCH STREET & 615 & M & 45 \\
\hline HARTMAN'S REAL ESTATE & 805 & PACIFIC AVENUE & 615 & M & 29 \\
\hline BEACH HILL REAL ESTATE + HARTMAN'S RE & 805 & PACIFIC AVENUE & 615 & M & 29 \\
\hline MISSION REALTY & 515 & CEDARSTREET & 615 & M & 20 \\
\hline HOUSTON WALTER REALTY & 420 & FRONT STREET & 615 & M & 17 \\
\hline LOTTS AUTO UPHOL & 820 & PACIFIC AVENUE & 641 & M & 512 \\
\hline PROLO CHEVROLET BODY SHOP - repairs & 412 & FRONT STREET & 641 & M & 161 \\
\hline HOLIDAY MUFFLER SERV AUTO REPAIR & 314 & FRONT STREET & 641 & $\mathbf{M}$ & 23 \\
\hline DEL MAR THEATRE & 1124 & PACIFIC AVENUE & 721 & M & 784 \\
\hline SC BOWLING & 1011 & PACIFIC AVENUE & 741 & $M$ & 583 \\
\hline NUMBEROFM & & 115 & & & \\
\hline TOTAL M2 OF M & & 40140 & & & \\
\hline AVE M2 OF M & & 349 & & & \\
\hline$\%$ OF M (AREA) & & 74.5 & & & \\
\hline SDFORM & & 426 & & & \\
\hline
\end{tabular}




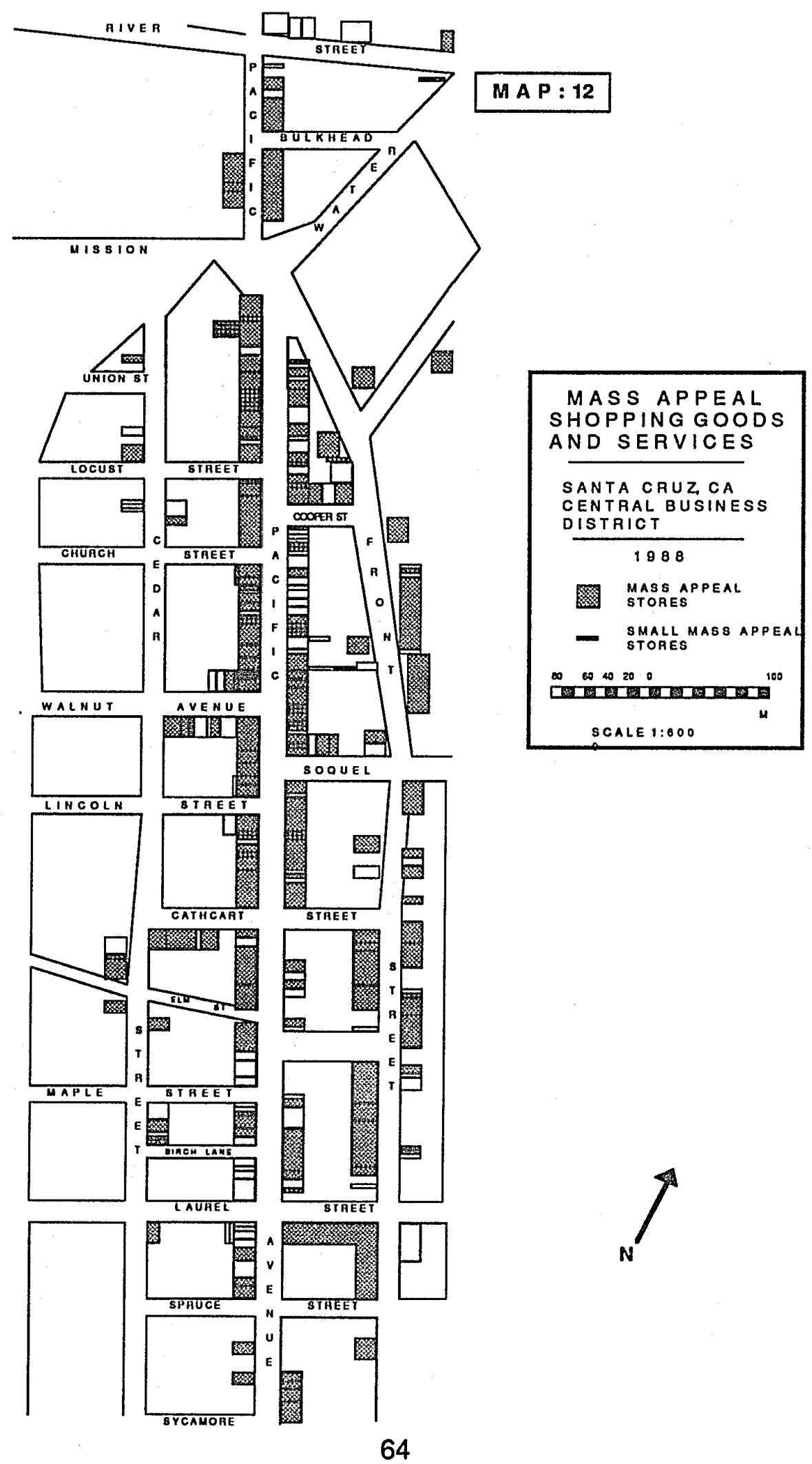


TABLE 18: MASS APPEAL STORES IN SANTA CRUZ IN 1988 BY STANDARD LAND USE CODE

\begin{tabular}{|c|c|c|c|c|c|}
\hline Name of Establishement & Streo & t Address & $\begin{array}{l}\text { Stand } \\
\text { Land L } \\
\text { Code }\end{array}$ & & \\
\hline GREYHOUND BUS & 423 & FRONT STREET & 421 & $\mathbf{M}$ & 344 \\
\hline RIODAN-WINNETT TRAVEL & 1338 & PACIFIC AVENUE & 492 & $\mathbf{M}$ & 106 \\
\hline SANTA CRUZ TRAVEL & 1519 & PACIFIC AVENUE & 492 & $\mathbf{M}$ & 101 \\
\hline CACACE + VICTORINO - plumbing + heating & 413 & FRONT STREET & 522 & $M$ & 382 \\
\hline CIMARELLI'S PLUMBING CO. & 209 & LAUREL STREET & 522 & $M$ & 63 \\
\hline KELLY-MOORE PAINTS & 941 & PACIFIC AVENUE & 523 & $M$ & 659 \\
\hline SANTA CRUZ HARDWARE & 1523 & PACIFIC AVENUE & 525 & $\mathbf{M}$ & 580 \\
\hline FORD'S & 1101 & PACIFIC AVENUE & 531 & M & 1736 \\
\hline WOOLWORTHS & 1213 & VENUE & 531 & $M$ & 706 \\
\hline$J C P I$ & 231 & ST & 531 & M & 445 \\
\hline narket & 700 & STREET & 541 & $M$ & 1786 \\
\hline - CANDY COLL & 110 & TREET & 544 & $M$ & 35 \\
\hline BAKERY & 609 & JENUE & 546 & $M$ & 225 \\
\hline BAGE & 320 & REET & 546 & $M$ & 162 \\
\hline DN SHOP - & 1014 & ENUE & 546 & $M$ & 142 \\
\hline NCHPASTRY & 1547 & ENUE & 546 & $M$ & 61 \\
\hline PLAZ & 1540 & VENUE & 546 & $\mathbf{M}$ & 43 \\
\hline OCEANCH & 555 & VENUE & 551 & $M$ & 3154 \\
\hline TOYC & 201 & FRC & 551 & $M$ & 2896 \\
\hline dealer & 130 & TREET & 551 & $M$ & 822 \\
\hline AUTO & 513 & VENUE & 551 & $M$ & 252 \\
\hline ENTER & 131 & AEET & 552 & M & 692 \\
\hline KRAGEI & 703 & REET & 552 & $M$ & 265 \\
\hline STERIO + UPHOLST. & 600 & IVENUE & 552 & M & 240 \\
\hline - parts & 901 & VENUE & 552 & M & 214 \\
\hline - tires & 703 & IENUE & 552 & M & 212 \\
\hline 's clothes & 1308 & IENUE & 561 & M & 233 \\
\hline MAMA - & 1100 & JENUE & 562 & M & 569 \\
\hline clothes & 1329 & IENUE & 562 & $M$ & 248 \\
\hline $\mathrm{J}$ - women's cl & 813 & VENUE & 562 & $M$ & 165 \\
\hline - women's clothes2 & 1350 & VENUE & 562 & M & 137 \\
\hline J-women's clothes & 1345 & VVENUE & 562 & M & 122 \\
\hline ANGE & 1373 & AVENUE & 562 & M & 113 \\
\hline - women's clothes & 1220 & AVENUE & 562 & M & 112 \\
\hline IE - clothes & 717 & IENUE & 562 & M & 60 \\
\hline TRADER TOTS & 2030 & ENUE & 564 & $M$ & 359 \\
\hline ANIMAL CRACKERS - children's shoes & 1325 & PACIFIC AVENUE & 564 & M & 248 \\
\hline COTTON TAILES - children's clothes & 810 & AVENUE & 564 & $M$ & 159 \\
\hline GOTTSCHALDS - clothes & 1405 & PACIFIC AVENUE & 565 & M & 2235 \\
\hline clothes & 1312 & PACIFIC AVENUE & 565 & M & 642 \\
\hline LOTU & 1541 & IVENUE & 565 & M & 358 \\
\hline CHANG & 1301 & VENUE & 565 & M & 313 \\
\hline FACTORY OUTLET - clothes & 125 & WALNUT AVENUE & 565 & $M$ & 257 \\
\hline CHI - PANTS & $\begin{array}{c}1521 \\
65\end{array}$ & PACIFIC AVENUE & 565 & $M$ & 250 \\
\hline
\end{tabular}


EXPRESS - clothes

SHANDRYDAN -clothes, variety

TRIAD - clothes

BODY OPTIONS - clothes

CHI PANTS

PANTS PLUS - clothes

PACIFIC PACK + PANTS

TROJENZ LTD. - clothes

COGNITO - clothes

TENGGARA - clothes

EARTH ANGEL - clothes

WESTERN VISIONS - clotheS

CAT 'N' CANERY - clothes

E POINT - clothes

MAX DAKOTA - clothes

CLOTHES HORSE - used clothes

JACKSON'S SHOES AND THINGS

PAYLESS SHOE SOURCE

ATHLETIC X-PRESS SHOE FACTORY

BUNNY'S SHOES

KEN'S SHOES

HAT CO., THE

SOCKS SHOP SANTA CRUZ

MCMAHAN'S FURNITURE

HABERS APPLIANCE SERV. CENTER - furn.

HABER'S - furniture

SPODICKS GENERAL FURNITURE $C O$.

RUNDYAN'S - furniture

INSIDE - furniture

ID BUILDING - furniture

APPLIANCE SERVICE CENTER

SINGER

SOUNDWAVES - stereo

CATALYST - reSt

ACAPULCO MEXICAN REST.

HEINZ BIERGARTEN CAFE

PIZZACO.

CREPEPLACE

BENTEN - japanese rest

REBECCA'S MIGHTY MUFFINS - coffee

CHINA SZECHWAN RESTURANT

CAFE CAMELEON

EMI - KOREAN CUISENE

GATSBY'S - rest

ZACHARY'S - rest

GARAGE GRILL

YESTERDAYS - deli

POTATO WORKS - rest

SCHLOTZSKY'S - rest

BUBBLE CAFE

$\begin{array}{lllll}1387 & \text { PACIFIC AVENUE } & 565 & \mathrm{M} & 247 \\ 107 & \text { COOPERSTREET } & 565 & \mathrm{M} & 179 \\ 1115 & \text { PACIFIC AVENUE } & 565 & \mathrm{M} & 174 \\ 1379 & \text { PACIFIC AVENUE } & 565 & \mathrm{M} & 150 \\ 121 & \text { WALNUT AVENUE } & 565 & \mathrm{M} & 143 \\ 1367 & \text { PACIFIC AVENUE } & 565 & \mathrm{M} & 132 \\ 1216 & \text { PACIFIC AVENUE } & 565 & \mathrm{M} & 112 \\ 1516 & \text { PACIFIC AVENUE } & 565 & \mathrm{M} & 112 \\ 821 & \text { PACIFIC AVENUE } & 565 & \mathrm{M} & 106 \\ 1533 & \text { PACIFIC AVENUE } & 565 & \mathrm{M} & 100 \\ 1535 & \text { PACIFIC AVENUE } & 565 & \mathrm{M} & 100 \\ 1539 & \text { PACIFIC AVENUE } & 565 & \mathrm{M} & 95 \\ 1510 & \text { PACIFIC AVENUE } & 565 & \mathrm{M} & 92 \\ 1547 & \text { PACIFIC AVENUE } & 565 & \mathrm{M} & 77 \\ 1210 & \text { PACIFIC AVENUE } & 565 & \mathrm{M} & 69 \\ 507 & \text { CEDARSTREET } & 565 & \mathrm{M} & 52 \\ 1306 & \text { PACIFIC AVENUE } & 566 & \mathrm{M} & 209 \\ 1218 & \text { PACIFIC AVENUE } & 566 & \mathrm{M} & 205 \\ 1125 & \text { PACIFIC AVENUE } & 566 & \mathrm{M} & 187 \\ 1512 & \text { PACIFIC AVENUE } & 566 & \mathrm{M} & 169 \\ 1537 & \text { PACIFIC AVENUE } & 566 & \mathrm{M} & 70 \\ 1120 & \text { PACIFIC AVENUE } & 569 & \mathrm{M} & 56 \\ 1521 & \text { PACIFIC AVENUE } & 569 & \mathrm{M} & 35 \\ 333 & \text { FRCNT STREET } & 571 & \mathrm{M} & 1105 \\ 428 & \text { FRONT STREET } & 571 & \mathrm{M} & 1019 \\ 812 & \text { PACIFIC AVENUE } & 571 & \mathrm{M} & 992 \\ 1001 & \text { PACIFIC AVENUE } & 571 & \mathrm{M} & 800 \\ 440 & \text { FRONT STREET } & 571 & \mathrm{M} & 758 \\ 428 & \text { FRONT STREET } & 571 & \mathrm{M} & 534 \\ 1515 & \text { PACIFIC AVENUE } & 571 & \mathrm{M} & 466 \\ 428 & \text { FRONT STREET } & 572 & \mathrm{M} & 136 \\ 113 & \text { WALNUT AVENUE } & 572 & \mathrm{M} & 132 \\ \mathbf{5 1 8} & \text { FRONT STREET } & 573 & \mathrm{M} & 289 \\ 1011 & \text { PACIFIC AVENUE } & 581 & \mathrm{M} & 1166 \\ 1118 & \text { PACIFIC AVENUE } & 581 & \mathrm{M} & 692 \\ 1335 & \text { PACIFIC AVENUE } & 581 & \mathrm{M} & 573 \\ 429 & \text { FRONT STREET } & 581 & \mathrm{M} & 484 \\ 2037 & \text { PACIFIC AVENUE } & 581 & \mathrm{M} & 453 \\ 1541 & \text { PACIFIC AVENUE } & 581 & \mathrm{M} & 358 \\ 514 & \text { FRONT STREET } & 581 & \mathrm{M} & 327 \\ 221 & \text { CATHCART ST } & 581 & \mathrm{M} & 310 \\ 418 & \text { FRONT STREET } & 581 & \mathrm{M} & 306 \\ 1003 & \text { CEDAR STREET } & 581 & \mathrm{M} & 293 \\ 821 & \text { FRONT STREET } & 581 & \mathrm{M} & 287 \\ \mathbf{8 1 7} & \text { PACIFIC AVENUE } & 581 & \mathrm{M} & 279 \\ 1417 & \text { PACIFIC AVENUE } & 581 & \mathrm{M} & 258 \\ 1360 & \text { PACIFIC AVENUE } & 581 & \mathrm{M} & 254 \\ 115 & \text { COOPER AVENUE } & 581 & \mathrm{M} & 216 \\ & & & \\ 1530 E T & 581 & \mathrm{M} & 201 \\ & & & & \end{array}$




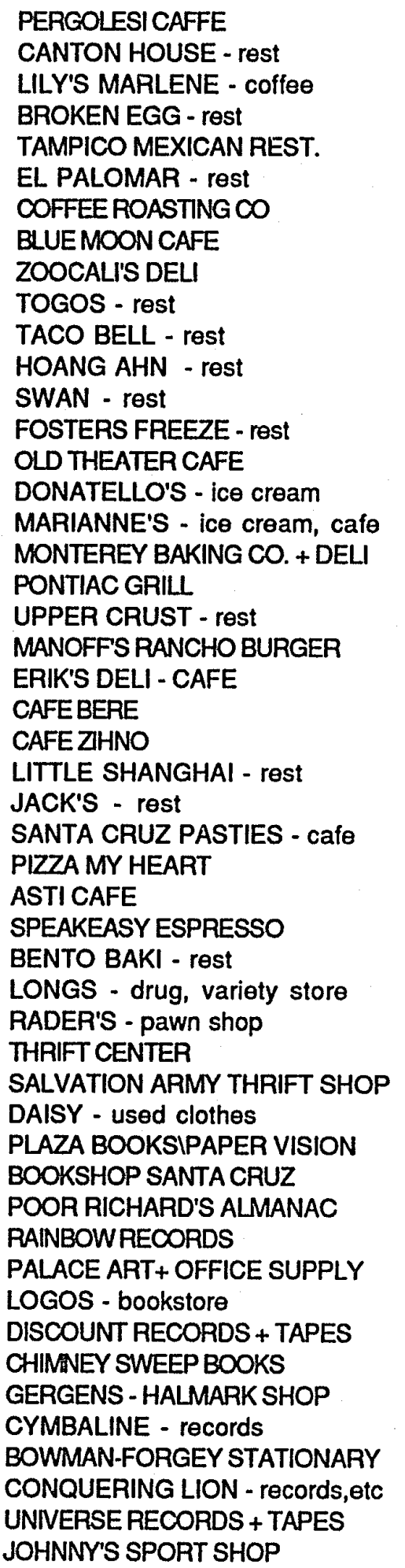

\begin{tabular}{|c|c|c|c|c|}
\hline 18 & CEDAR STREET & 581 & M & 187 \\
\hline 617 & PACIFIC AVENUE & 581 & & \\
\hline 1363 & PACIFIC AVENUE & 581 & & \\
\hline 05 & FRONT STREET & 581 & & \\
\hline 22 & PACIFIC AVENUE & 581 & & \\
\hline 1344 & PACIFIC AVENUE & 581 & & \\
\hline 1549 & PACIFIC AVENUE & 581 & & \\
\hline 1110 & PACIFIC AVENUE & 581 & & . \\
\hline 1534 & PACIFIC AVENUE & 581 & & 13 \\
\hline 1213 & PACIFIC AVENUE & 581 & & . \\
\hline 802 & PACIFIC AVENUE & 581 & & 12 \\
\hline 2019 & PACIFIC & 581 & & 12 \\
\hline 1538 & PACIFIC AVENUE & 581 & 101 & \\
\hline 229 & CEDARSTREET & 581 & $M$ & \\
\hline 106 & WALNUT & 581 & $M$ & 11 \\
\hline 713 & PACIFICA & 581 & $M$ & 11 \\
\hline 107 & SOQUEL AVENUE & 581 & $\mathbf{M}$ & 10 \\
\hline 920 & PACIFIC AVENUE & 581 & $\mathbf{M}$ & $v$ \\
\hline 429 & FRONTST & 581 & $M$ & 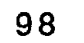 \\
\hline 1012 & PACIFIC AVENUE & 581 & on & 31 \\
\hline 142 & WATER S1 & 581 & $M$ & 91 \\
\hline 712 & FRONT ST & 58 & $M$ & 89 \\
\hline 1101 & CEDARST & 58 & $M$ & 83 \\
\hline 1547 & PACIFIC AVENUE & 581 & $M$ & 81 \\
\hline 1547 & PACIFIC A & 581 & $M$ & 77 \\
\hline 202 & CED/ & 58 & $M$ & 75 \\
\hline 4 & PALOMAF & 58 & $M$ & 62 \\
\hline 111 & SOQUELA & 58 & M & 60 \\
\hline 715 & LAURELS & 58 & M & 50 \\
\hline$r$ & PALOMAF & 58 & $M$ & 45 \\
\hline 110 & EET & 58 & $M$ & 35 \\
\hline 600 & FRO & & M & 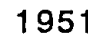 \\
\hline 1209 & PACIFIC A & 59 & $M$ & 8 \\
\hline 504 & FRONT STREET & 59 & $M$ & 64 \\
\hline 322 & FRONT STREET & 593 & $N$ & 12 \\
\hline 817 & FRONT STREET & 593 & 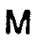 & 52 \\
\hline 1111 & PACIFICA & 594 & M & 726 \\
\hline 1547 & PACIFIC A & 594 & $M$ & 640 \\
\hline 521 & FRONT STREET & 594 & M & 503 \\
\hline 1411 & PACIFIC AVENUE & 594 & M & 468 \\
\hline 1224 & PACIFIC AVENUE & 59 & M & 43 \\
\hline 1117 & PACIFIC AVENUE & 594 & $M$ & 302 \\
\hline 1207 & PACIFIC AVENUE & 594 & $M$ & 259 \\
\hline 419 & CEDAR STREET & 594 & $M$ & 249 \\
\hline 1201 & PACIFIC AVENUE & 594 & M & 242 \\
\hline 435 & FRONT STREET & 594 & $M$ & 211 \\
\hline 1319 & PACIFIC AVENUE & 594 & $\mathbf{M}$ & 123 \\
\hline 705 & PACIFIC AVENUE & 594 & N & 100 \\
\hline 1215 & PACIFIC AVENUE & 594 & 10 & 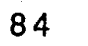 \\
\hline 1017 & PACIFIC AVENUE & 595 & M & 304 \\
\hline
\end{tabular}




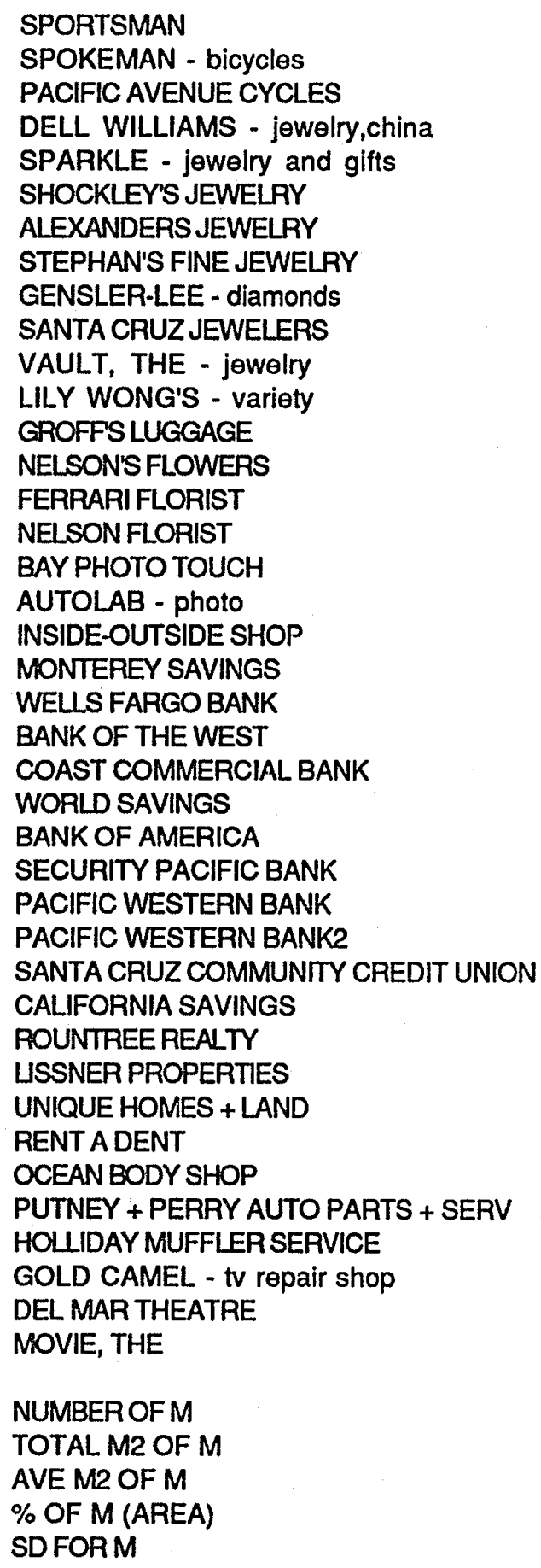

$\begin{array}{lllll}1532 & \text { PACIFIC AVENUE } & 595 & \mathrm{M} & 242 \\ 501 & \text { CEDARSTREET } & 595 & \mathrm{M} & 232 \\ 709 & \text { PACIFIC AVENUE } & 595 & \mathrm{M} & 205 \\ 1320 & \text { PACIFIC AVENUE } & 597 & \mathrm{M} & 388 \\ 1205 & \text { PACIFIC AVENUE } & 597 & \mathrm{M} & 177 \\ 1359 & \text { PACIFIC AVENUE } & 597 & \mathrm{M} & 172 \\ 2046 & \text { PACIFIC AVENUE } & 597 & \mathrm{M} & 139 \\ 102 & \text { WALNUT AVENUE } & 597 & \mathrm{M} & 106 \\ 1123 & \text { PACIFIC AVENUE } & 597 & \mathrm{M} & 88 \\ 1122 & \text { PACIFIC AVENUE } & 597 & \mathrm{M} & 56 \\ 1341 & \text { PACIFIC AVENUE } & 597 & \mathrm{M} & 53 \\ 1129 & \text { PACIFIC AVENUE } & 599 & \mathrm{M} & 925 \\ 1384 & \text { PACIFIC AVENUE } & 599 & \mathrm{M} & 154 \\ 235 & \text { CATHCART ST } & 599 & \mathrm{M} & 285 \\ 1222 & \text { PACIFIC AVENUE } & 599 & \mathrm{M} & 150 \\ 235 & \text { CEDAR STREET } & 599 & \mathrm{M} & 128 \\ 117 & \text { WALNUT AVENUE } & 599 & \mathrm{M} & 178 \\ 119 & \text { WALNUT AVENUE } & 599 & \mathrm{M} & 125 \\ 910 & \text { CEDARSTREET } & 599 & \mathrm{M} & 90 \\ 530 & \text { FRONT STREET } & 611 & \mathrm{M} & 1083 \\ 74 & \text { RIVERSTREET } & 611 & \mathrm{M} & 862 \\ 2020 & \text { PACIFIC AVENUE } & 611 & \mathrm{M} & 665 \\ 701 & \text { FRONT STREET } & 611 & \mathrm{M} & 622 \\ 1551 & \text { PACIFIC AVENUE } & 611 & \mathrm{M} & 620 \\ 1128 & \text { PACIFIC AVENUE } & 611 & \mathrm{M} & 458 \\ 104 & \text { RIVERSTREET } & 611 & \mathrm{M} & 344 \\ 1502 & \text { PACIFIC AVENUE } & 611 & \mathrm{M} & 304 \\ 25 & \text { RIVERSTREET } & 611 & \mathrm{M} & 253 \\ 512 & \text { FRONT STREET } & 611 & \mathrm{M} & 228 \\ 1208 & \text { PACIFIC AVENUE } & 611 & \mathrm{M} & 207 \\ 320 & \text { CEDARSTREET } & 615 & \mathrm{M} & 75 \\ 320 & \text { CEDAR STREET } & 615 & \mathrm{M} & 75 \\ 107 & \text { CHURCH STREET } & 615 & \mathrm{M} & 45 \\ 512 & \text { PACIFIC AVENUE } & 639 & \mathrm{M} & 175 \\ 555 & \text { PACIFIC AVENUE } & 641 & \mathrm{M} & 825 \\ 325 & \text { FRONT STREET } & 641 & \mathrm{M} & 435 \\ 309 & \text { FRONT STREET } & 641 & \mathrm{M} & 237 \\ 1529 & \text { PACIFIC AVENUE } & 649 & \mathrm{M} & 105 \\ 1124 & \text { PACIFIC AVENUE } & 721 & \mathrm{M} & 784 \\ 324 & \text { FRONT STREET } & 721 & \mathrm{M} & 157\end{array}$

184

62212

338

79.0

443 
Automobile traffic has been limited on the "main street", and plush greenery has been planted in an attempt to encourage pedestrian strolling. The growth of retail space on the "main street" was only a modest $8.4 \%$, while the total CBD retail area grew by $46.2 \%$. The percent of the total area that is on the "main street" decreased over sixteen percent, from $62.2 \%$ to $46.1 \%$. Over $80 \%$ of the "main street" retail area is now used by mass appeal stores. As expected, the "main street" has a slightly smaller percentage of limited appeal space. The percentage of convenience space on the "main street" is approximately the same for the "main street" as it is for the whole CBD: about $5 \%$. 


\section{WATSONUILLE}

\section{$1960-1988$}

Watsonville is located in Santa Cruz county, south of the city of Santa Cruz. The Central Business District in Watsonville consists of about six blocks along Main Street, from Riverside Drive to Ford Street. It extends to the east and to the west from one to two blocks perpendicular to Main Street. (see map 13)

The population in Watsonville was 13,290 in 1960, larger than either Hollister or Los Gatos, but smaller than Santa Cruz. Although the population of Watsonville was slightly more than twice the population of Hollister, the total retail space in the downtown was only about $45 \%$ larger. One quarter of the retail stores in Watsonville in 1960, were located outside of the CBD. (see table 2) From 1960 to 1988 the population of Watsonville more than doubled to 28,550 people. However, the downtown retail space decreased, indicating a retail growth outside of the downtown area. The average size store increased by $17 \mathrm{~m}^{2}$. The size of the average store on the "main street" also increased due to an influx of a few very large mass appeal stores.

Convenience Space (see tables 19-22)

More than $15 \%$ of the total retail space was taken up by convenience stores. Convenience stores frequently occupied corner locations. About $50 \%$ of the possible corner locations throughout the downtown were taken up by convenience stores which accounted for only $15 \%$ of the total space. The convenience stores were distributed 


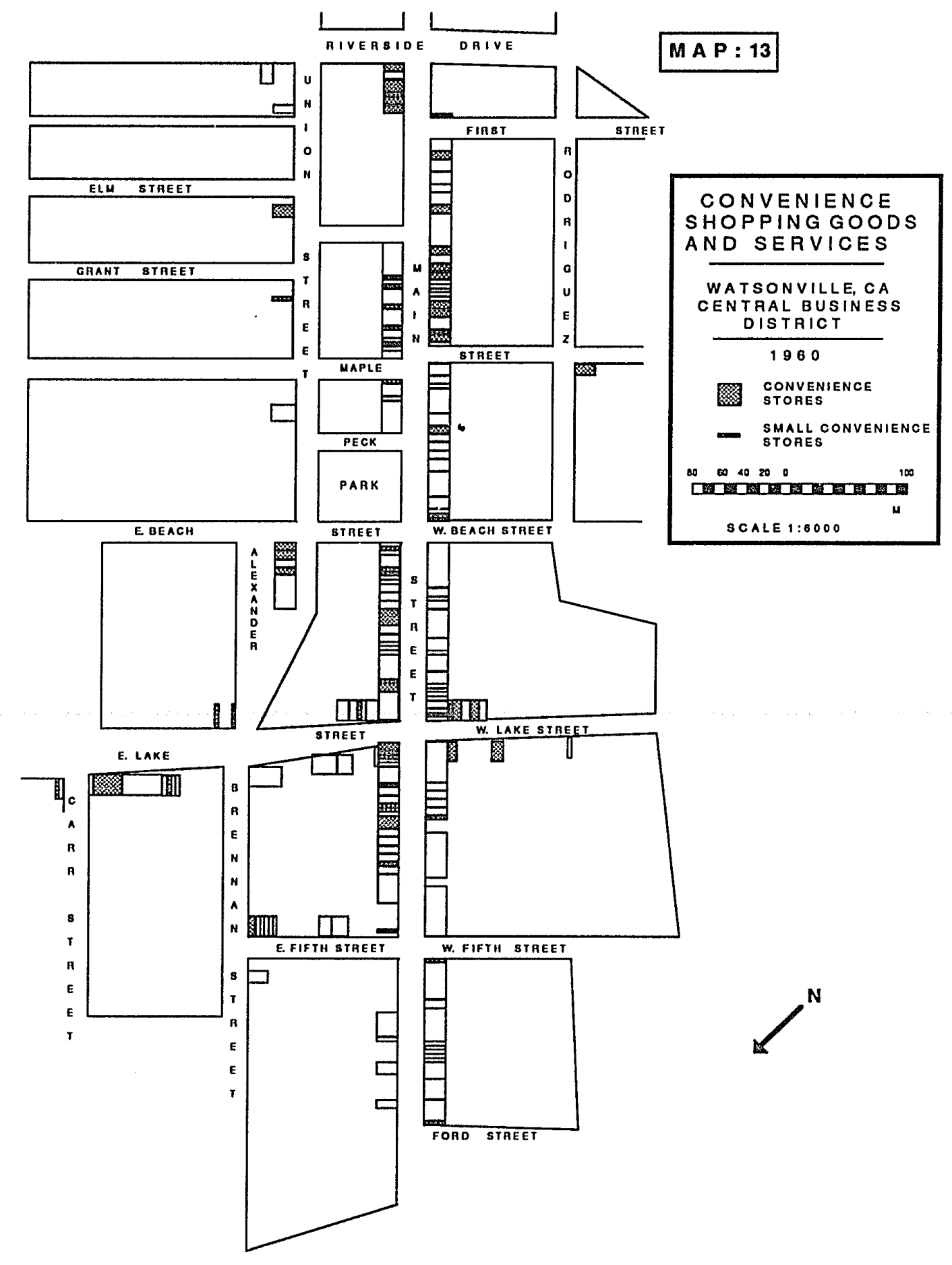


TABLE 19: STATISTICS FOR WATSONVILLE'S CBD

\section{STATISTICS OF WATSONVILLE 1960}

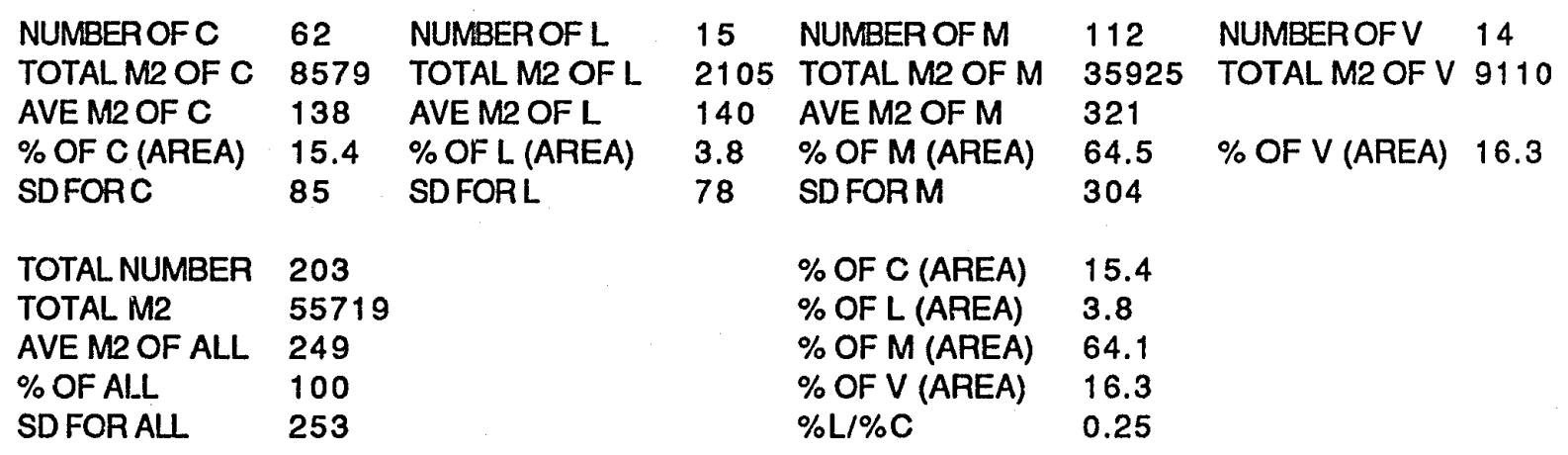

STATISTICS FOR WATSONVILLE 1988

$\begin{array}{llllllll}\text { NUMBER OF C } & 39 & \text { NUMBER OFL } & 29 & \text { NUMBER OFM } & 113 & \text { NUMBEROFV } & 15 \\ \text { TOTAL M2 OF C } & 4865 & \text { TOTAL M2 OF L } & 4454 & \text { TOTAL M2 OF M } & 39261 & \text { TOTAL M2 OF V } 3538 \\ \text { AVE M2 OF C } & 125 & \text { AVE M2 OF L } & 154 & \text { AVE M2 OF M } & 347 & \\ \text { \% OF C (AREA) } & 9.3 & \% \text { OF L (AREA) } & 8.5 & \% \text { OF M (AREA) } & 75.3 & \% \text { OF V (AREA) } & 6.8 \\ \text { SD FORC } & 84 & \text { SDFORL } & 110 & \text { SD FORM } & 409 & \\ & & & & \% \text { OF C (AREA) } & 9.3 & \\ \text { TOTAL NUMBER } & 198 & & & \% \text { OF L (AREA) } & 8.5 & \\ \text { TOTAL M2 } & 52118 & & \% \text { OF M (AREA) } & 75.3 & \\ \text { AVE M2 OF ALL } & 266 & & & \% \text { OF V (AREA) } & 6.8 & \\ \text { \% OF ALL } & 100 & & & \% \text { (AR } \% \text { C } & 0.91 & \\ \text { SD FOR ALL } & 335 & & & \end{array}$

CHANGE IN STATISTICS FOR WATSONVILLE'S CBD

\begin{tabular}{|c|c|c|c|c|c|c|}
\hline $\begin{array}{l}\text { CH IN \# C } \\
\text { CH M2 OF C } \\
\text { CH AVE M2 OF C } \\
\text { CH \% OF C AREA }\end{array}$ & $\begin{array}{l}-23 \\
-3714 \\
-13 \\
-6.1\end{array}$ & $\begin{array}{l}\text { CH IN \# L } \\
\text { CH M2 OF L } \\
\text { CH AVE M2 OF L } \\
\text { CH \% OF L AREA }\end{array}$ & $\begin{array}{l}14 \\
2349 \\
14 \\
4.7\end{array}$ & $\begin{array}{l}\mathrm{CH} \text { IN \# M } \\
\text { CH M2 OF M } \\
\text { CH AVE M2 OF M } \\
\text { CH \% OF M AREA }\end{array}$ & $\begin{array}{l}1 \\
3336 \\
26 \\
17.8\end{array}$ & $\begin{array}{ll}\text { CH IN \# V } & 1 \\
\text { CH M2 OF V } & -5572 \\
\text { CH } \% \text { OF V AREA }-9.5\end{array}$ \\
\hline $\begin{array}{l}\text { CH TOTAL NBR } \\
\text { CH TOTAL M2 } \\
\text { CH AVE M2 ALL }\end{array}$ & $\begin{array}{l}-5 \\
-3601 \\
17\end{array}$ & & $\% \mathrm{CHI}$ & $\begin{array}{l}\% \text { CH IN \% OF C } \\
\% \text { CH IN \% OF L } \\
\% \text { CH IN \% OFM } \\
\text { IN \% OF V }\end{array}$ & $\begin{array}{l}-39.6 \\
123.7 \\
16.7 \\
-58.4\end{array}$ & \\
\hline$\% \mathrm{CH}$ IN TOT M2 & -6.5 & & $\mathrm{CH}$ IN & $\% L / \% C$ & 0.67 & \\
\hline
\end{tabular}


TABLE 20: STATISTICS FOR WATSONVILL'S MAIN STREET

STATISTICS FOR WATSONVILLE'S MAIN STREET 1960

\begin{tabular}{|c|c|c|c|c|c|c|c|}
\hline $\begin{array}{l}\text { NUMBER OF C } \\
\text { TOTAL M2 OF C } \\
\text { AVE M2 OF C } \\
\% \text { OF C (AREA) } \\
\text { SD FORC }\end{array}$ & $\begin{array}{l}43 \\
6112 \\
142 \\
15.8 \\
90\end{array}$ & $\begin{array}{l}\text { NUMBER OFL } \\
\text { TOTAL M2 OF L } \\
\text { AVE M2 OF L } \\
\% \text { OF L (AREA) } \\
\text { SDFORL }\end{array}$ & $\begin{array}{l}10 \\
1331 \\
133 \\
3.4 \\
79\end{array}$ & $\begin{array}{l}\text { NUMBER OFM } \\
\text { TOTAL M2 OF M } \\
\text { AVE M2 OF M } \\
\% \text { OF M (AREA) } \\
\text { SD FORM }\end{array}$ & $\begin{array}{l}83 \\
28304 \\
341 \\
73.0 \\
323\end{array}$ & $\begin{array}{l}\text { NUMBER OFV } \\
\text { TOTAL M2 OF V }\end{array}$ & $\begin{array}{l}11 \\
3038\end{array}$ \\
\hline $\begin{array}{l}\text { TOTAL NUMBER } \\
\text { TOTAL M2 } \\
\text { AVE M2 OF ALL } \\
\% \text { OF ALL } \\
\text { SD FOR ALL }\end{array}$ & $\begin{array}{l}152 \\
38785 \\
262 \\
100 \\
269\end{array}$ & & & $\begin{array}{l}\% \text { OF } \% \text { (AREA) } \\
\% \text { OF L (AREA) } \\
\% \text { OF M (AREA) } \\
\% \text { OF V (AREA) } \\
\% \text { L } \% \text { C }\end{array}$ & $\begin{array}{l}15.8 \\
3.4 \\
73.0 \\
7.8 \\
\\
0.22\end{array}$ & & \\
\hline
\end{tabular}

\% AREA ON MAIN 69.6

STATISTICS FOR WATSONVILLE'S MAIN STREET 1988

\begin{tabular}{|c|c|c|c|c|c|c|c|}
\hline $\begin{array}{l}\text { NUMBER OFC } \\
\text { TOTAL M2 OF C }\end{array}$ & $\begin{array}{l}18 \\
2300\end{array}$ & $\begin{array}{l}\text { NUMBER OF L } \\
\text { TOTAL M2 OF L }\end{array}$ & $\begin{array}{l}18 \\
2739\end{array}$ & $\begin{array}{l}\text { NUMBER OF M } \\
\text { TOTAL M2 OF M }\end{array}$ & $\begin{array}{l}71 \\
25999\end{array}$ & $\begin{array}{l}\text { NUMBER OFV } \\
\text { TOTAL M2 OF V }\end{array}$ & $\begin{array}{l}11 \\
1906\end{array}$ \\
\hline AVE M2 OF C & 128 & AVE M2 OF L & 152 & AVE M2 OF M & 366 & & \\
\hline $\begin{array}{l}\% \text { OF C (AREA) } \\
\text { SDFORC }\end{array}$ & $\begin{array}{l}7.0 \\
91\end{array}$ & $\%$ OF L (AREA) & 8.3 & $\%$ OF M (AREA) & $\begin{array}{l}78.9 \\
474\end{array}$ & $\%$ OF V (AREA) & 5.8 \\
\hline TOTAL NUMBER & 118 & & & $\%$ OF C (AREA) & 7.0 & & \\
\hline TOTAL M2 & 32944 & & & $\%$ OF L (AREA) & 8.3 & & \\
\hline AVE M2 OF ALL & 279 & & & $\%$ OF $M$ (AREA) & 78.9 & & \\
\hline$\%$ OF ALL & 100 & & & $\%$ OF V (AREA) & 5.8 & & \\
\hline SD FOR ALL & 388 & & & $\% L / \% C$ & 1.19 & & \\
\hline
\end{tabular}

$\%$ AREA ON MAIN 63.2

CHANGE IN WATSONVILLE'S MAIN STREET 1960-1988

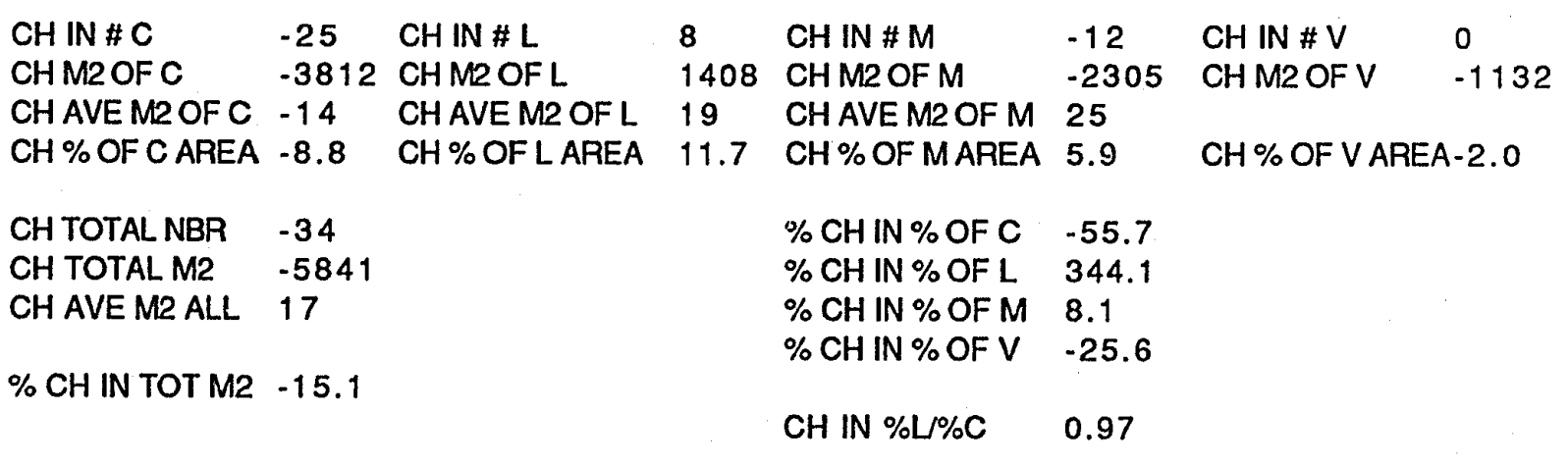


TABLE 21 : CONVENIENCE STORES IN WATSONVILLE IN 1960 BY STANDARD LAND USE CODE

\begin{tabular}{|c|c|c|c|c|c|c|}
\hline \multirow{2}{*}{$\begin{array}{l}\text { Name of Establishement } \\
\text { FARMER'S MARKET - grocery }\end{array}$} & & \multicolumn{2}{|c|}{ Street Address } & $\begin{array}{l}\text { Standard } \\
\text { Land Use } \\
\text { Code }\end{array}$ & Type & Area $M^{2}$ \\
\hline & & 321 & RODRIGUEZST & 541 & C & 296 \\
\hline DAYLITE MKT GRO & $m * *$ & 235 & MAIN STREET & 541 & c & 232 \\
\hline EAKER BROS FOOD MART & & 30.5 & E. 5TH STREET & 541 & C & 124 \\
\hline BIG FIVE SERVICE STATION - gas & 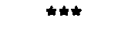 & 102 & E. LAKE AVE & 553 & C & 76 \\
\hline BERGMAN MOBIL - gas & $\star \star \star \star$ & 134 & E. LAKE AVE & 553 & C & 76 \\
\hline FRANK'S CHEVRON - gas & $\star \star \star \star$ & 222 & MAIN STREET & 553 & C & 76 \\
\hline GENE'S UNION SERV. & $\star \star \star \star$ & 369 & MAIN STREET & 553 & C & 76 \\
\hline BOKARIZA SHELL - gas & $* \star \star *$ & 601 & MAIN STREET & 553 & C & 76 \\
\hline COX CHEVRON - gas & & 189 & MAIN STREET & 553 & C & 35 \\
\hline B+H FLYING A - gas station & & 570 & MAIN STREET & 553 & C & 15 \\
\hline MCKIBBEN'S MOBILE SERV & & 655 & MAIN STREET & 553 & C & 15 \\
\hline TAYLOR'S HOT DOG STAND & & 22 & MAPLE AVE & 581 & C & 18 \\
\hline CORRAL THE - bar & & 436 & MAIN STREET & 582 & C & 471 \\
\hline TOWNE CLUB - bar & & 538 & MAIN STREET & 582 & C & 339 \\
\hline BUSY BEE TAVERN & & 259 & MAIN STREET & 582 & C & 238 \\
\hline CAESAR'S TAVERN - bar & & 184 & MAIN STREET & 582 & C & 201 \\
\hline EL PALOMAR CLUB - bar & $\star \star \star \star$ & 190 & MAIN STREET & 582 & C & 170 \\
\hline EL. PALOMAR POOL HALL & $* * *$ & 192 & MAIN STREET & 582 & C & 170 \\
\hline UNIVERSAL BAR & *** & 205 & MAIN STREET & 582 & C & 170 \\
\hline CENTREAL CLUB TAVERN & *** & 240 & MAIN STREET & 582 & C & 170 \\
\hline MY PLACE - bar & \#\#\# & 250 & MAIN STREET & 582 & C & 170 \\
\hline PALACE BAR AND GRILL - bar & $* * *$ & 269 & MAIN STREET & 582 & C & 170 \\
\hline PALACE CLUB ROOM & $m * *$ & 269 & MAIN STREET & 582 & C & 170 \\
\hline REX CLUB TAVERN & "*** & 277 & MAIN STREET & 582 & C & 170 \\
\hline ROYAL CLUB TAVERN & *** & 420 & MAIN STREET & 582 & C & 170 \\
\hline LARRY'S COCKTAIL LOUNGEIREST & $* * *$ & 10 & W. LAKE ST & 582 & C & 170 \\
\hline PAJARO ROOM COCKTAIL LOUNGE & $* \star \star *$ & 13 & W. LAKE ST & 582 & C & 170 \\
\hline CLUB MIRAMAR - bar & & 522 & MAIN STREET & 582 & C & 130 \\
\hline PAJARO GOLD - bar & & 17 & W. LAKE ST & 582 & C & 121 \\
\hline MARK, THE - bar & & 24 & E. LAKE ST & 582 & C & 96 \\
\hline PROGRESS CLUB TAVERN & & 506 & MAIN STREET & 582 & C & 75 \\
\hline LOG CABIN - bar & & 534 & MAIN STREET & 582 & C & 65 \\
\hline JOHNSONS DRUGS & & 500 & MAIN STREET & 591 & C & 251 \\
\hline STEIHAUSER + EATON DRUGS & & 325 & MAIN STREET & 591 & C & 230 \\
\hline MAY WAY PHARM & \#** & 139 & E. LAKE AVE & 591 & C & 222 \\
\hline HUMBLE DRUG CO & $\star \star \star \star$ & 247 & MAIN STREET & 591 & C & 222 \\
\hline PRESCRIPTION PHARMACY & & 408 & MAIN STREET & 591 & C & 102 \\
\hline CORNERDRUGSTORE & & 359 & MAIN STREET & 591 & C & 85 \\
\hline PAJARO VALLEY FAMILY LIQUOR & $* * *$ & 16 & W. LAKE ST & 592 & C & 289 \\
\hline GREEN CELLARLIQUORS & & 251 & MAIN STREET & 592 & C & 215 \\
\hline KOKKA LIQUOR STORE & & 180 & MAIN STREET & 592 & C & 152 \\
\hline WATSONVILLE LIQUORSTORE & & 11.5 & ALEXANDER ST & 592 & C & 92 \\
\hline OLNERIUS CLEANERS & & 460 & MAIN STREET & 621 & C & 265 \\
\hline
\end{tabular}




\begin{tabular}{|c|c|c|c|c|c|c|}
\hline WATSONVILLE LNDRY + DRY CLN & RS *** & 212 & MAIN STREET & 621 & C & 139 \\
\hline SEQUOIA LNDRY + DRY CLEANER & $\star \star \star \star$ & 18 & MAPLE AVE & 621 & C & 139 \\
\hline DO-IT YOURSELF LAUNDRY & & 123 & E. LAKE ST & 621 & $\mathrm{C}$ & 123 \\
\hline PARISIAN CLEANERS & & 11 & ALEXANDER ST & 621 & $\mathrm{C}$ & 114 \\
\hline PLAZA-PARIS BEAUTY SALON & $\star \star \star \star$ & 27 & ALEXANDER ST & 623 & $\mathrm{C}$ & 96 \\
\hline CALIFORNIA BARBER SHOP & *** & 255.5 & MAIN STREET & 623 & C & 96 \\
\hline STAR BARBER SHOP & *** & 260 & MAIN STREET & 623 & C & 96 \\
\hline ROYAL BARBER SHOP & *** & 422 & MAIN STREET & 623 & C & 96 \\
\hline MAIN BARBER SHOP & *** & 552 & MAIN STREET & 623 & C & 96 \\
\hline RESETAR BEAUTY SALON & $\star \star \star \star$ & 9 & W. LAKE ST & 623 & C & 96 \\
\hline ADKINS BARBER SHOP & & 458.5 & MAIN STREET & 623 & C & 95 \\
\hline DORIS BEAUTY SHOP & & 228 & UNION STREET & 623 & C & 85 \\
\hline DELUXE BARBER SHOP & & 504 & MAIN STREET & 623 & C & 75 \\
\hline ODA BARBER SHOP & & 186 & MAIN STREET & 623 & C & 71 \\
\hline BLUE BIRD BARBER SHOP & & 530 & MAIN STREET & 623 & C & 65 \\
\hline KUT N' KURL - beauty & & 209 & E. LAKE ST & 623 & C & 64 \\
\hline RESETAR BARBER SHOP & & 465 & MAIN STREET & 623 & C & 40 \\
\hline HELTON'S SHINE SHOP & *** & 275.5 & MAIN STREET & 625 & C & 71 \\
\hline HERMAN'S CAR WASH & $* \star *$ & 535 & MAIN STREET & 641 & $\mathrm{C}$ & 76 \\
\hline NUMBEROF C & & & 62 & & & \\
\hline TOTAL M2 OF C & & & 8579 & & & \\
\hline AVE M2 OF C & & & 138 & & & \\
\hline$\%$ OF C (AREA) & & & 15.4 & & & \\
\hline SDFORC & & & 85 & & & \\
\hline
\end{tabular}


TABLE 22: CONVENIENCE STORES IN WATSONVILLE IN 1988 BY STANDARD LAND USE CODE

\begin{tabular}{|c|c|c|c|c|c|}
\hline Name of Establishement & Street & Address & $\begin{array}{l}\text { Standard } \\
\text { Land Use } \\
\text { Code }\end{array}$ & Type & Area $\mathrm{M}^{2}$ \\
\hline ELCHARRITO GROSERY & 29 & ALEXANDER ST & 541 & C & 329 \\
\hline DAILY MEAT MARKET - grocery & 329 & RODRIGUEZST & 541 & $\mathrm{C}$ & 153 \\
\hline PAJARO VALLEY FISH MARKET & 114 & UNION STREET & 541 & c & 67 \\
\hline CHEVRON - gas & 189 & MAIN STREET & 553 & C & 20 \\
\hline MOBIL - gas & 570 & MAIN STREET & 553 & C & 15 \\
\hline LA FUENTE - rest & 5 & MAPLE AVE & 581 & $\mathrm{C}$ & 37 \\
\hline HOTDOGS & 22 & MAPLE AVE & 581 & $\mathrm{C}$ & 18 \\
\hline LA FRONTERA CLUB - bar & 178 & MAIN STREET & 582 & C & 322 \\
\hline PASA TIEMPO CLUB - bar & 259 & MAIN STREET & 582 & C & 238 \\
\hline EL BOHEMIO CLUB - bar & 249 & MAIN STREET & 582 & $\mathrm{C}$ & 215 \\
\hline CAESAR'S CLUB - bar & 184 & MAIN STREET & 582 & C & 201 \\
\hline LA POBLANITA - bar & 180.5 & MAIN STREET & 582 & $\mathrm{C}$ & 152 \\
\hline EL TENAMPA II - bar & 24 & E. LAKE ST & 582 & $\mathrm{C}$ & 96 \\
\hline JOHNSON'S DRUGS & 500 & MAIN STREET & 591 & $\mathrm{C}$ & 251 \\
\hline BEACH STREET VIDEO & 13 & E. BEACHST & 592 & $\mathrm{C}$ & 128 \\
\hline OTI VIDEO RENTAL & 313 & MAIN STREET & 592 & C & 120 \\
\hline INNER CITY VIDEO & 25 & W. LAKE ST & 592 & $\mathrm{C}$ & 119 \\
\hline 5TH STREET LAUNDROMAT & 18 & E. 5TH STREET & 621 & C & 273 \\
\hline IDEAL DRY CLEANERS & 200 & UNION STREET & 621 & C & 263 \\
\hline E. LAKE WASH AND DRY - laundry & 359 & E. LAKE ST & 621 & $\mathrm{C}$ & 123 \\
\hline COIN LAUNDRY & 107 & UNION STREET & 621 & C & 115 \\
\hline TOWNTRY FASHIONS & 548 & MAIN STREET & 623 & C & 207 \\
\hline ANA'S BEAUTY SALON & 28 & W. BEACH ST & 623 & c & 175 \\
\hline YUAI BEAUTY SALON & 115 & E. LAKE ST & 623 & C & 150 \\
\hline UNIQUE HAIR STYLIST SALON & 443 & MAIN STREET & 623 & $\mathrm{c}$ & 109 \\
\hline CHICO + FRIENDS CUTTING HAIR & 408 & MAIN STREET & 623 & $\mathrm{C}$ & 102 \\
\hline VOGUE HAIR DESIGN & 25 & E. LAKE ST & 623 & C & 97 \\
\hline DORIS BEAUTY SHOP & 228 & UNIONSTREET & 623 & $\mathrm{C}$ & 85 \\
\hline LUPES HOUSE OF BEAUTY & 3 & W. BEACHST & 623 & $\mathrm{C}$ & 84 \\
\hline HAIR'S THE PLACE & 23 & W. LAKE ST & 623 & C & 72 \\
\hline ODA BARBER SHOP & 186 & MAIN STREET & 623 & C & 71 \\
\hline MARY'S BEAUTY SALON & 629 & MAIN STREET & 623 & C & 66 \\
\hline SILVER SHEARS & 30 & E. 5TH STREET & 623 & $\mathrm{C}$ & 65 \\
\hline KUTN'KURL & 209 & E. LAKE ST & 623 & $\mathrm{C}$ & 64 \\
\hline BARBER SHOP & 267 & MAIN STREET & 623 & $\mathrm{C}$ & 63 \\
\hline VI'S BEAUTY SALON & 540 & MAIN STREET & 623 & C & 61 \\
\hline LEONARDO'S STYLING & 521 & MAIN STREET & 623 & C & 47 \\
\hline RESETARBARBER SHOP & 465 & MAIN STREET & 623 & $\mathrm{C}$ & 40 \\
\hline NORMS SHOE SERVICE & 11 & W. BEACHST & 625 & $\mathrm{C}$ & 52 \\
\hline NUMBEROFC & & 39 & & & \\
\hline TOTAL M2 OF C & & 4865 & & & \\
\hline AVE M2 OF C & & 125 & & & \\
\hline$\%$ OF C (AREA) & & 9.3 & & & \\
\hline SDFORC & & 84 & & & \\
\hline
\end{tabular}


through out the downtown. Almost all of the blocks have at least one convenience store. There was a small clustering of convenience stores on the south end of Main Street by Riverside Drive. The small block between Alexander Street and Union Street was also dominated by convenience stores. (see map 13)

By 1988 , the retail area in convenience stores decreased to $9.3 \%$, a decrease of more than a third. The drop in convenience space was even greater for the "main street", which decreased to only $7.0 \%$. (see fig 3) Gas stations, bars, liquor stores, and drug stores all decreased in importance. In fact, no liquor stores remain in the downtown today. The percentage of beauty salons and barber shops increased from $11.4 \%$ to $32.0 \%$. (see table 22 ). The area used by corner markets and laundromats changed only slightly. The clustering of convenience stores at the south end of Main Street still exists in 1988, but only one store on Alexander Street is still a convenience type store. (see map 14)

\section{Limited Appeal Space}

Limited appeal stores only took up $3.8 \%$ of the total floorspace in 1960. The ratio of limited appeal space to convenience space was .25 for the downtown as a whole and .22 for the "main street". The percentage of space limited appeal stores took up per block ranged from $0 \%$ to $63 \%$, but the great majority had less than $10 \%$. The blocks with high percentages of limited appeal were off the "main street" or on the very edge of the downtown. (see map 15) Very few of the limited appeal stores in 1960 appealed particularly to the wealthy. 
Fig. 3

Percentages of retail land use in the CBD WATSONVILLE

WATSONVILLE 1960

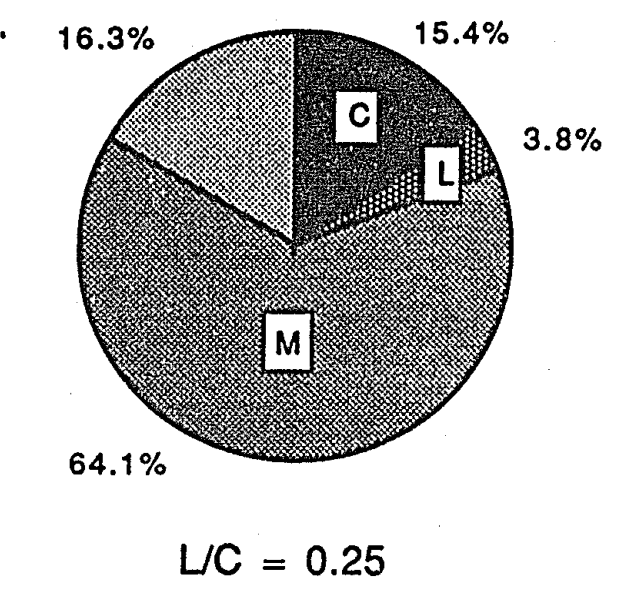

WATSONVILLE 1988

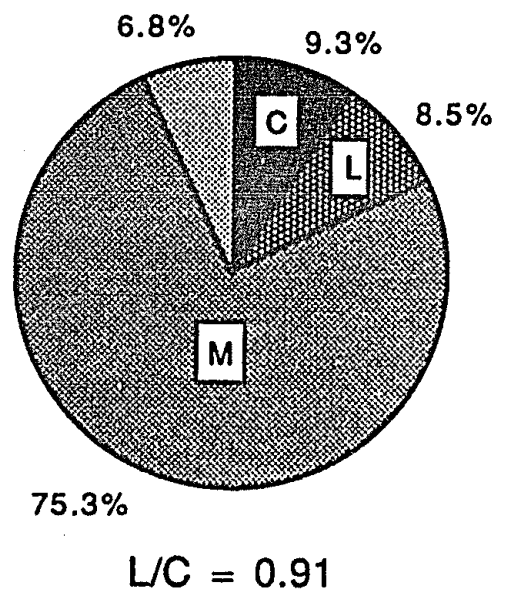

WATSONVILLE 1960 THE MAIN ST ONLY

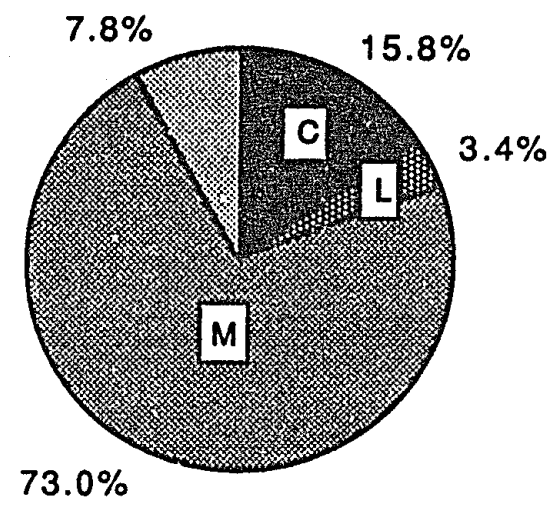

$$
L C=0.22
$$

WATSONVILLE 1988 THE MAIN ST ONLY

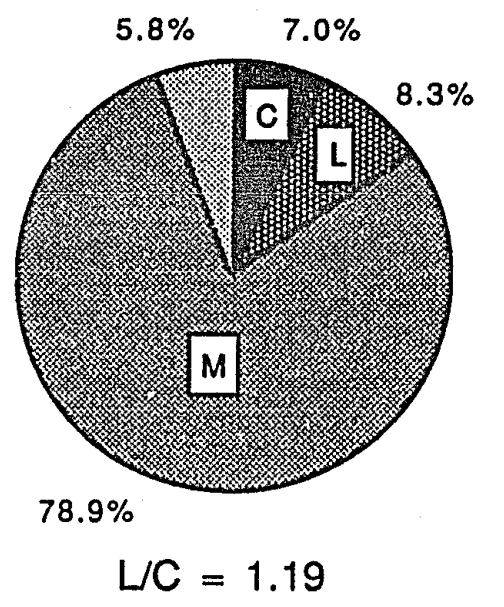




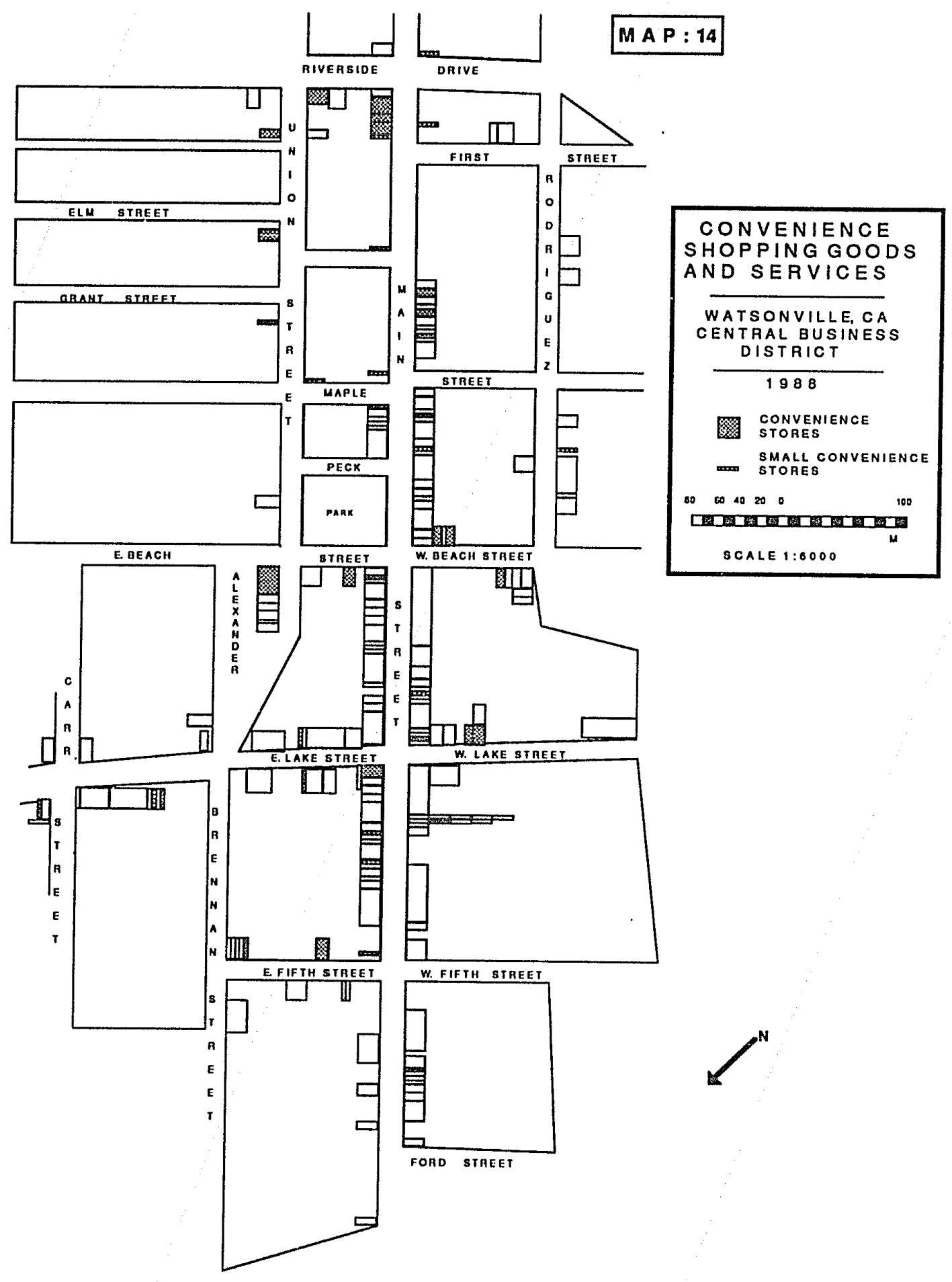




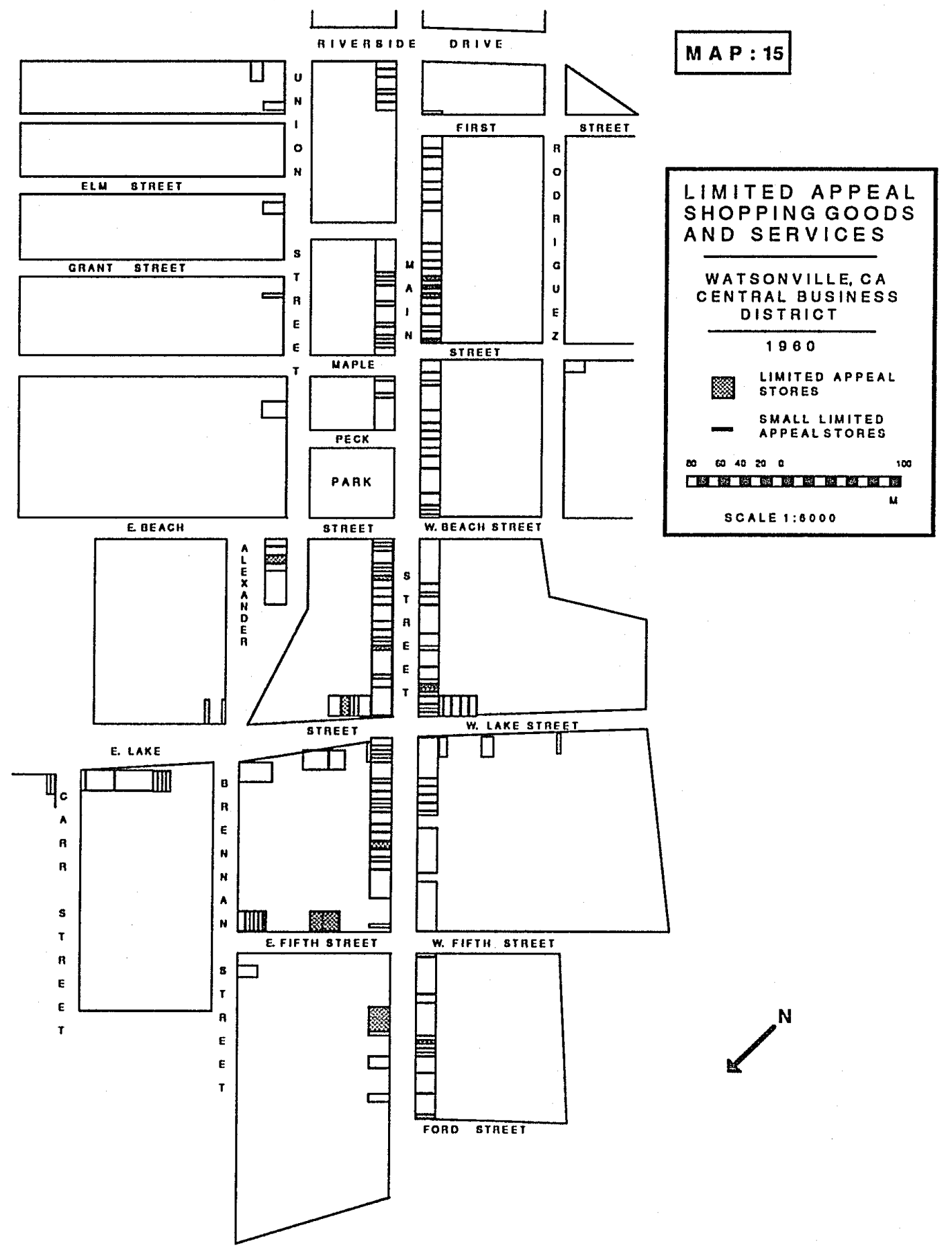


The two largest limited appeal siores were glass stores. The only stores that even came close to being luxury type stores were one antique shop, a couple of camera stores, and a gift shop. Three of the limited appeal stores are cigar stores, which tend to be on the convenience side of limited appeal. (see table 23)

Space in limited appeal stores more than doubled during this time period. The ratio of limited appeal space to convenience space increased to .91 for the downtown as a whole and to 1.19 for Main Street. The new shopping area in La Manzana, which was built in from 521 Main Street along a walkway, now has several limited appeal stores. (see map 16) One would expect this type of development to attract limited appeal stores because, although they are not really on the main street, they have a main street address. One of the stores here is a Reggae shop which seems too limited for Watsonville today. It has only opened recently and its success is doubtful. Stores with extremely limited appeal are still rare in Watsonville. For instance, there are no antique shops here. (see table 24)

\section{Mass Appeal Space (map 17)}

Sixty-four percent of the total retail space consisted of mass appeal stores. (see fig. 3) These stores ranged in size from only 16 square meters to 1814 square meters. The average was $321 \mathrm{~m}^{2}$, more than twice the average of the other types. In 1960, there were nine auto dealerships within the CBD. Six of them were even located on the "main street". It is difficult to tell how large they were, because more than half of them have been torn down and replaced. 
TABLE 23 : LIMITED APPEAL STORES IN WATSONVILLE IN 1960 BY STANDARD LAND USE CODE

\begin{tabular}{|c|c|c|c|c|c|}
\hline Name of Establishement & Street & Address & $\begin{array}{l}\text { Standard } \\
\text { Land Use } \\
\text { Code }\end{array}$ & Туре & Area $M^{2}$ \\
\hline WESTERN UNON TELEG CO. & 30 & E. 5TH STREET & 442 & L & 65 \\
\hline WATSONVILE GLASS CO & 630 & MAIN STREET & 523 & $\mathbf{L}$ & 330 \\
\hline COAST GLASS CO & 18 & E. 5TH STREET & 523 & $\mathbf{L}$ & 273 \\
\hline GOLDEN RICHSHA CATERERS & 11D & ALEXANDER ST & 549 & $\mathbf{L}$ & 109 \\
\hline SCHMAUS LUDWIG - tailor & 28 & E. LAKE AVE & 567 & $\mathbf{L}$ & 120 \\
\hline CACTUS SPORTLAND SHOOTING GAL & 261 & MAIN STREET & 573 & $\mathbf{L}$ & 115 \\
\hline TWO SIXTY FIVE CLUB - card room & 265 & MAIN STREET & 582 & $\mathbf{L}$ & 100 \\
\hline COOTS ART SMOKE SHOP & 267.5 & MAIN STREET & 592 & L & 63 \\
\hline GLAD'S THIS'N THAT SHOPPE - antiques & 22 & E. 5TH STREET & 593 & L & 207 \\
\hline A1 LOCK SHOP & 548 & MAIN STREET & 599 & $\mathrm{~L}$ & 207 \\
\hline STEPICK'S CAMERA SHOP & 450 & MAIN STREET & 599 & $\mathbf{L}$ & 130 \\
\hline SANTI'S CAMERA SHOP & 445 & MAIN STREET & 599 & L & 109 \\
\hline REX CIGAR STORE & 277.5 & MAIN STREET & 599 & L & 84 \\
\hline JACK'S CIGAR STORE & 424.5 & MAIN STREET & 599 & $\mathrm{~L}$ & 84 \\
\hline IDA'S GIFT SHOP & 443 & MAIN STREET & 599 & L & 109 \\
\hline NUMBER OFL & & 15 & & & \\
\hline TOTAL M2 OF L & & 2105 & & & \\
\hline AVE M2 OF L & & 140 & & & \\
\hline$\%$ OF L (AREA) & & 3.8 & & & \\
\hline SDFORL & & 78 & & & \\
\hline
\end{tabular}

*** Establishment assigned approximate value for area 


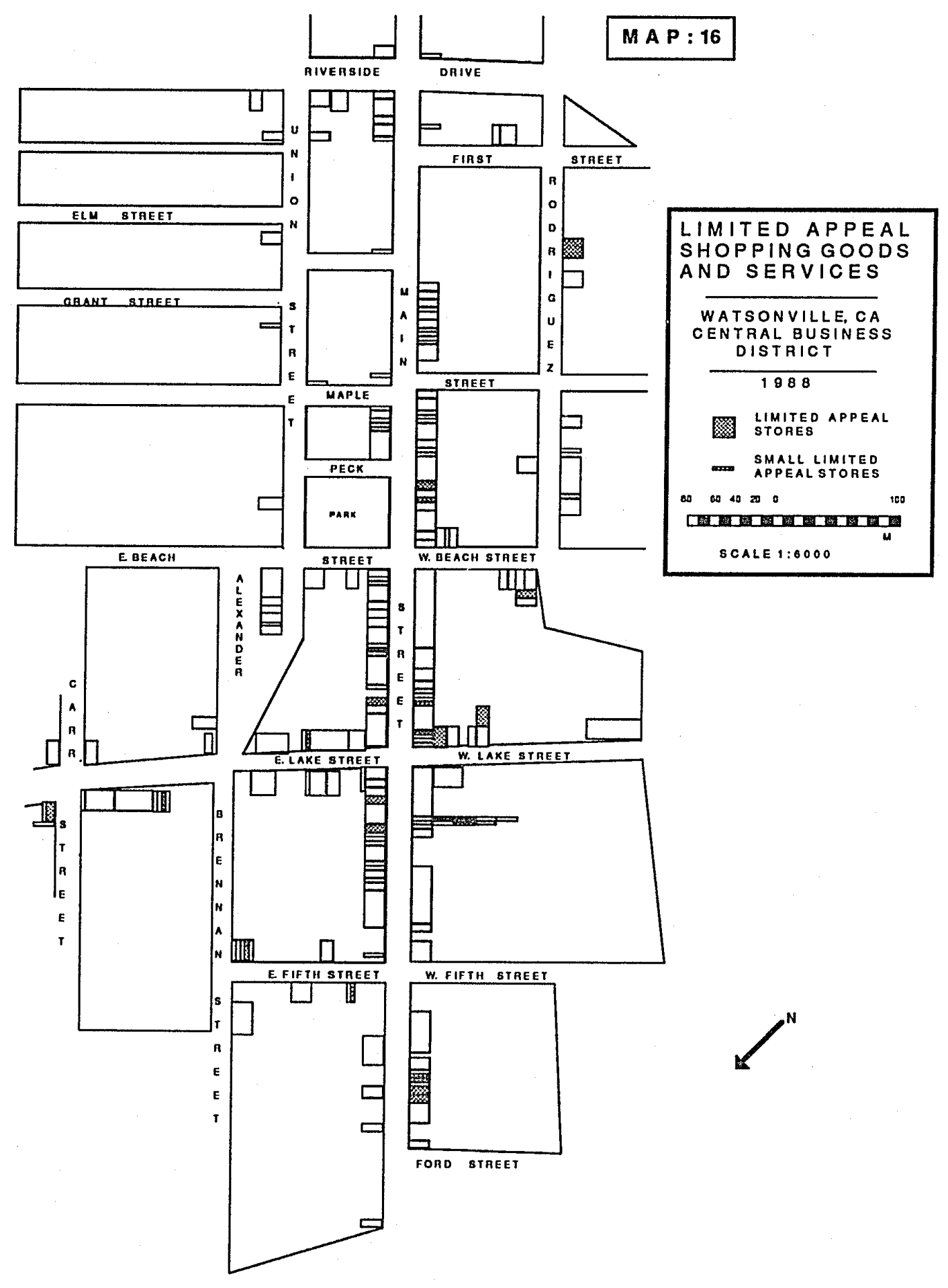


TABLE 24 : LIMITED APPEAL STORES IN WATSONVILLE IN 1988 BY STANDARD LAND USE CODE

\begin{tabular}{|c|c|c|c|c|c|}
\hline Name of Establishement & Streo & $t$ Address & $\begin{array}{l}\text { Standard } \\
\text { Land Use } \\
\text { Code }\end{array}$ & Type & Area $\mathrm{M}^{2}$ \\
\hline WESTERN UNION - telegraph & 629 & MAIN STREET & 472 & L & 66 \\
\hline HEALTHWAY - grocery & 117 & E. LAKE ST & 541 & $L$ & 153 \\
\hline ORIENTAL STORE & 205 & E. LAKE ST & 541 & $L$ & 71 \\
\hline MICHAEL'S OF MONTEREY BAY CATERING & 17 & W. LAKE ST & 549 & $L$ & 121 \\
\hline DAVIS AUTO PARTS - equip, mach shop & 245 & RODRIGUEZST & 552 & L & 518 \\
\hline TUXEDO WORLD & 27 & W. LAKE ST & 561 & L & 167 \\
\hline GOLDEN BUTTERFLY - boutique & $11 \mathrm{E}$ & ALEXANDER ST & 562 & L & 42 \\
\hline DORI'S BRIDAL WORLD & 337 & MAIN STREET & 563 & L & 260 \\
\hline TOUCH OF ELEGANCE - bridal & 518 & MAIN STREET & 563 & L & 153 \\
\hline BRIDAL VEIL & 447 & MAIN STREET & 563 & $\mathrm{~L}$ & 109 \\
\hline RELICULAS EN ESPANOL & 261 & MAIN STREET & 594 & $L$ & 233 \\
\hline JANSEN MUSIC & 446 & MAIN STREET & 594 & L & 117 \\
\hline CECILIA'S JEWELRY AND GIFTS & 521 & MAIN STREET & 597 & $\mathbf{L}$ & 45 \\
\hline STONEMOUNTAIN QUALITY FABRIC OUTLET & 410 & RODRIGUEZST & 599 & L & 168 \\
\hline WATSONVILLE HEARING AID CENTER & 600 & MAIN STREET & 599 & L & 70 \\
\hline STEPICK'S CAMERA SHOP & 459 & MAIN STREET & 599 & $\mathbf{L}$ & 241 \\
\hline FOTOGRAFIA WATSONVILLE & 521 & MAIN STREET & 599 & L & 47 \\
\hline BLOOSOMS AND BALOONS & 637 & MAIN STREET & 599 & $\mathrm{~L}$ & 165 \\
\hline DOUBLE PLAY BASEBALLCARDS +COMICS & $30 \mathrm{~A}$ & E. 5TH STREET & 599 & L & 65 \\
\hline REGGAESHOP & 521 & MAIN STREET & 599 & L & 45 \\
\hline MORRIS PLAN - loans, finance & 538 & MAIN STREET & 612 & $\mathrm{~L}$ & 339 \\
\hline CREDIT + COШECTION BUREAU OF WAT. & 321 & RODAIGUEZST & 612 & $L$ & 296 \\
\hline DORI'S PHOTOGRAPHY + FORMAL WEAR & 341 & MAIN STREET & 622 & $L$ & 214 \\
\hline TANNING $C O$. & 46 & BRENNANST & 629 & $L$ & 18 \\
\hline PENGUIN PRINTING & 510 & MAIN STREET & 633 & L & 139 \\
\hline RAPID OFFSET PRINTING & 22 & E. LAKE ST & 633 & L & 96 \\
\hline SHOP TOWEL RENTAL & 460 & MAIN STREET & 639 & $\mathrm{~L}$ & 265 \\
\hline U-HAUL RENTALS & 637 & MAIN STREET & 639 & L & 165 \\
\hline HENSLEY'S JEWELRY REPAIR & 629 & MAIN STREET & 649 & L & 66 \\
\hline NUMBER OF L & & 29 & & & \\
\hline TOTAL M2 OF L & & 4454 & & & \\
\hline AVE M2 OF L & & 154 & & & \\
\hline$\%$ OF L (AREA) & & 8.5 & & & \\
\hline SD FORL & & 110 & & & \\
\hline
\end{tabular}




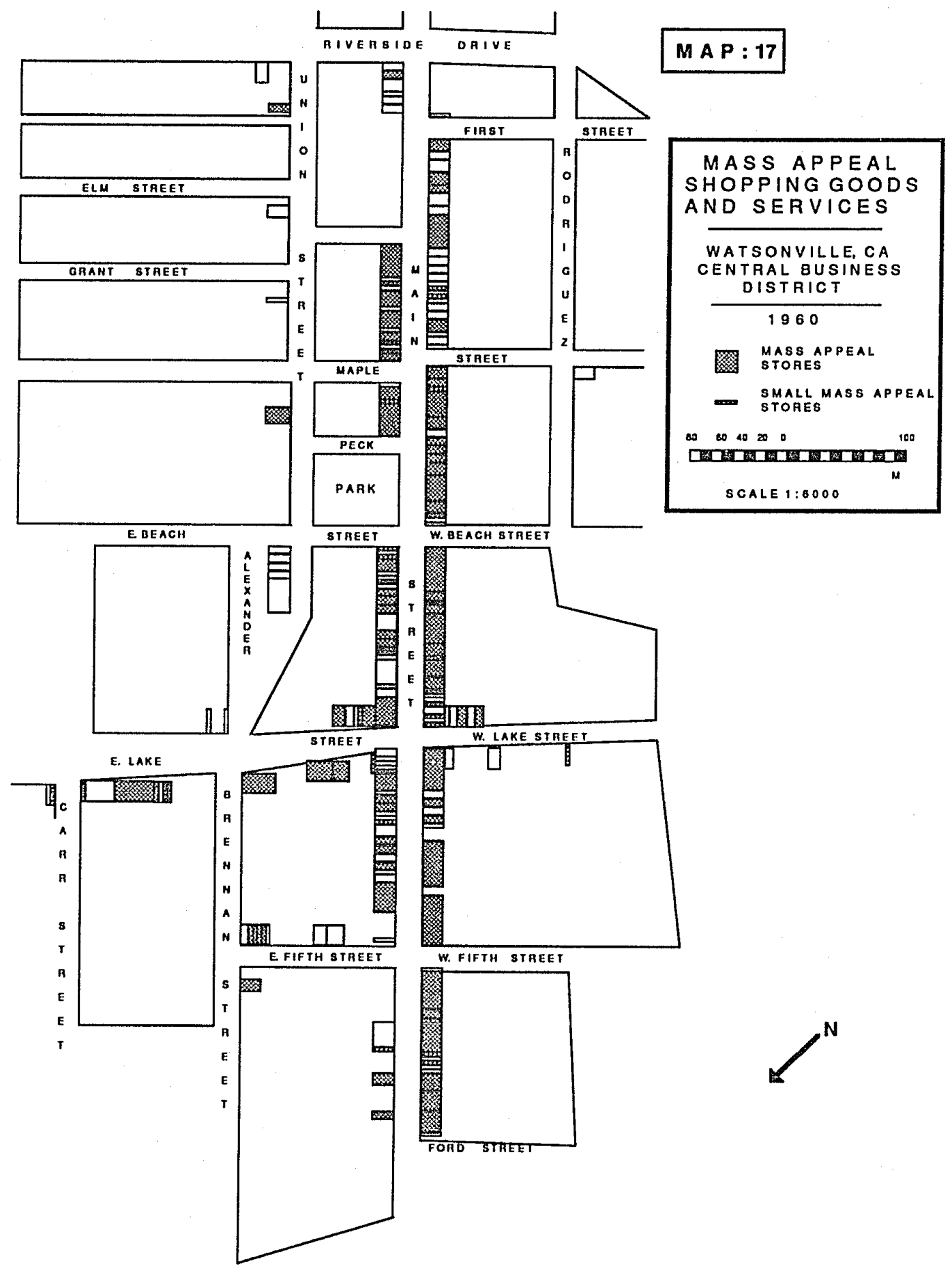


Mass appeal space increased from $64.1 \%$ to $75.3 \%$. (see map 18 ) Much of this increase is due to the decrease in vacancies. (see fig. 3) Restaurants increased modestly in importance, from $7.1 \%$ to $9.4 \%$. Banks, however, increased dramatically from only $2.3 \%$ of the total retail space to $11.5 \%$ of it. (see tables 25 and 26 )

\section{The Main Street (Main Street)}

All of Main Street, from Riverside Drive to Ford Street, is considered "the main street." In 1960, the only significant difference between Watsonville's whole downtown and Main Street, is the amount of vacant space. The downtown as a whole had $16.3 \%$ of the total retail space vacant, while the "main street" only had $7.8 \%$ of its retail space vacant. The stores off of the "main street" were larger on average than the stores on the "main street." $75 \%$ of the number of stores were on the "main street", while only $69.6 \%$ of the area was.

The main street had slightly less (under 1\%) limited appeal space and slightly more convenience space that the downtown as a whole did in 1960. The dominance of Main Street as the retail area has decreased in importance. In $1960,74.9 \%$ of the retail area in downtown was along Main Street. In 1988, it decreased to only $59.6 \%$. The non-main street expansion has been primarily along Rodriguez Street, and along the east-west streets. Near First Street, a considerable section of Main Street retail buildings has been torn down. The average size store on Main Street is slightly larger than for the whole of downtown because the absence of a shopping mall has promoted the development of large department and variety stores on the "main street". 


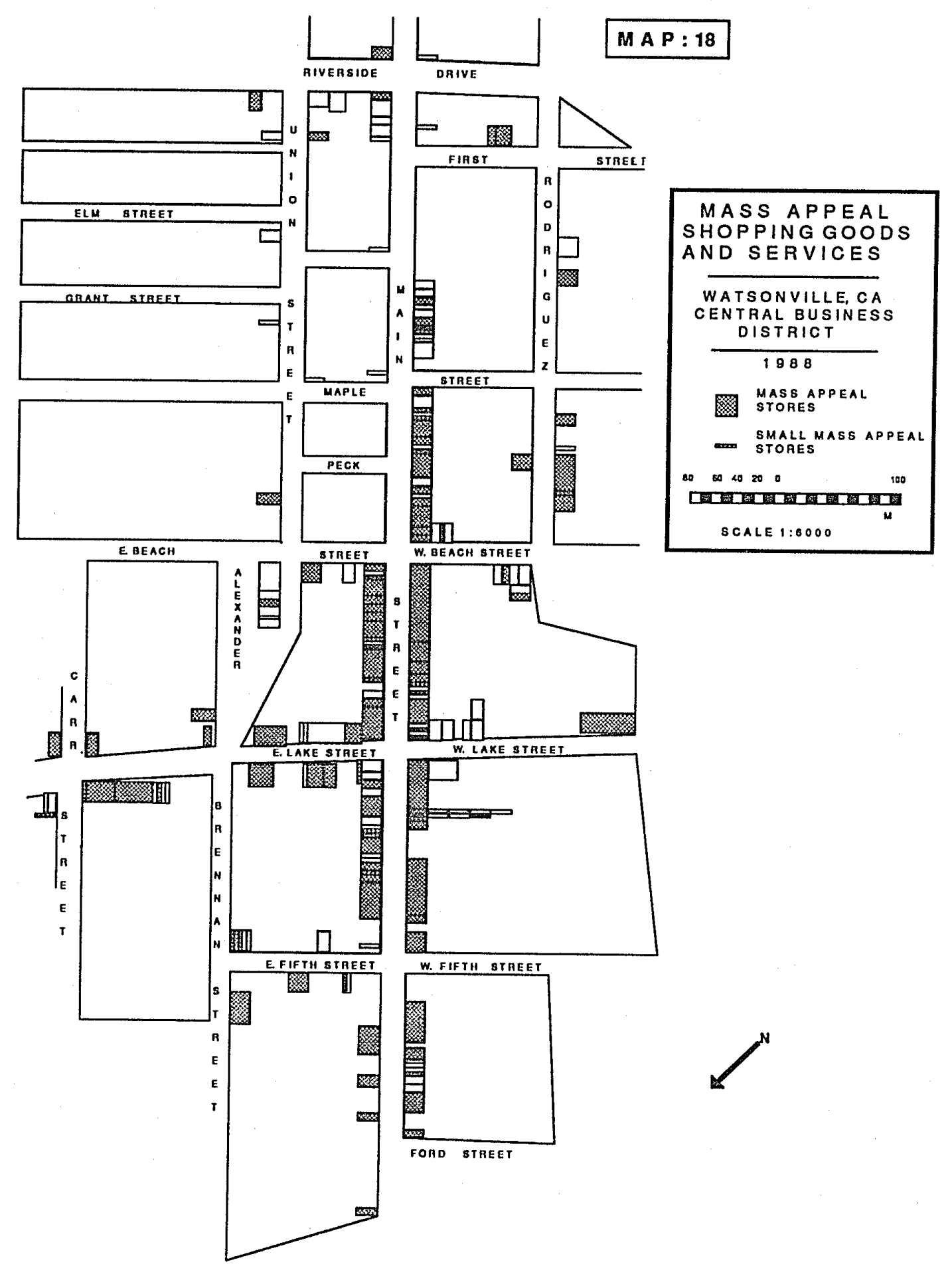


TABLE 25 : MASS APPEAL STORES IN WATSONVILLE IN 1960 BY STANDARD LAND USE CODE

\begin{tabular}{|c|c|c|c|c|c|}
\hline Name of Establishement & Street & Address & $\begin{array}{l}\text { Standard } \\
\text { Land Use } \\
\text { Code }\end{array}$ & Type & Area $\mathrm{M}^{2}$ \\
\hline GREENE TRAVELSERVICE & 21.6 & BRENNAN ST & 492 & M & 110 \\
\hline WATSONVILLE PAINT CENTER & 540 & MAIN STREET & 523 & M & 207 \\
\hline HARRISON'S COLOR CORNER - paint & 143 & E. LAKE ST & 523 & M & 67 \\
\hline ACE HARDWARE & 317 & MAIN STREET & 525 & M & 594 \\
\hline TREICHEL'S HDW + PLUMB & 315 & MAIN STREET & 525 & M & 45 \\
\hline J.C. PENNY & 501 & MAIN STREET & 531 & M & 1348 \\
\hline FORD'S DEPT STORE & 407 & MAIN STREET & 531 & M & 1176 \\
\hline RASCO F S + CO - dept store & 470 & MAIN STREET & 531 & M & 897 \\
\hline NATIONAL DOLLAR STORES & 349 & MAIN STREET & 531 & M & 681 \\
\hline WOOLWORTHS & 447 & MAIN STREET & 531 & M & 109 \\
\hline SEARS CALATALOG SALES & 429 & MAIN STREET & 532 & M & 260 \\
\hline LEW'S VARIETY STORE & 201 & MAIN STREET & 533 & M & 260 \\
\hline PURITY STORES LTD - grocery & 559 & MAIN STREET & 541 & M & 1814 \\
\hline PURITY STORES - grocery & 133 & E. LAKE ST & 541 & M & 920 \\
\hline WATSONVILLE LOCKERS - meat & 625 & MAIN STREET & 541 & M & 560 \\
\hline DALY BROS DRY GOODS & 345 & MAIN STREET & 541 & M & 329 \\
\hline BEATRICE FOODS INC & 519 & MAIN STREET & 541 & M & 260 \\
\hline PAC BROKERAGE CO. - frozen foods & 629 & MAIN STREET & 541 & M & 66 \\
\hline PANARO VALLEY FISH MARKET & 114 & UNIONSTREET & 542 & M & 67 \\
\hline MOOR'S CANDIE'S & 463 & MAIN STREET & 544 & M & 40 \\
\hline NEW YORK BAKERY & 218 & MAIN STREET & 546 & $\mathbf{M}$ & 165 \\
\hline PAJARO VALLEY BAKERY & 523 & MAIN STREET & 546 & $\mathbf{M}$ & 165 \\
\hline LAWSON IMPORTED CARS & 9 & E. 5TH STREET & 551 & M & 600 \\
\hline HADEN FORD SALES - used cars *** & 200 & MAIN STREET & 551 & M & 600 \\
\hline PORTOLA MOTORS AUTO DEALERS *** & 245 & MAIN STREET & 551 & M & 600 \\
\hline FRANICH CONTINENTAL LINCOLN *** & 566 & MAIN STREET & 551 & M & 600 \\
\hline ZAR MTRS USED CARS & 645 & MAIN STREET & 551 & M & 600 \\
\hline MOORIARTY CHEVROLET - car dealer & 35 & E. LAKE ST & 551 & M & 613 \\
\hline HANSEN PONTIAC - auto dealers & 640 & MAIN STREET & 551 & M & 494 \\
\hline ZAR MOTORS - auto dealers & 637 & MAIN STREET & 551 & M & 330 \\
\hline CROWLEY + TRAULSEN AUTOS & 25 & W. LAKE ST & 551 & M & 119 \\
\hline FERGUSON'S AUTO SUP & 516 & MAIN STREET & 552 & $M$ & 461 \\
\hline SWEET SERV - auto parts & 38 & ALEXANDER ST & 552 & M & 374 \\
\hline SETHEY'S TIRES & 40 & E. LAKE AVE & 552 & M & 374 \\
\hline WESTERN AUTO - parts & 439 & MAIN STREET & 552 & M & 278 \\
\hline ALEXANDER'S MEN'S CLOTHES & 353 & MAIN STREET & 561 & M & 334 \\
\hline SYLVAN'S MEN'S CLOTHES & 301 & MAIN STREET & 561 & M & 328 \\
\hline TOGGERY - men's clothes & 280 & MAIN STREET & 561 & M & 297 \\
\hline YOUNG'S MEN'S WEAR & 428 & MAIN STREET & 561 & M & 109 \\
\hline MORTON GENE MEN'S WEAR & 441 & MAIN STREET & 561 & M & 109 \\
\hline ANITA SHOP - women's clothes & 437 & MAIN STREET & 562 & M & 465 \\
\hline CLASSIC SHOP - women's clothes & 337 & MAIN STREET & 562 & M & 260 \\
\hline FASION THE - women's clothes & 427 & MAIN STREET & 562 & M & 159 \\
\hline JUSTIN APPAREL SHOP - women's clo & 412 & $\begin{array}{l}\text { MAIN STREET } \\
88\end{array}$ & 562 & M & 148 \\
\hline
\end{tabular}




\begin{tabular}{|c|c|c|c|c|c|}
\hline \multirow{2}{*}{$\begin{array}{l}\text { BARTLETT GENE - women's clothes } \\
\text { MODE O'DAY - women's clothes }\end{array}$} & 446 & MAIN STREET & 562 & $M$ & 117 \\
\hline & 471 & MAIN STREET & 562 & M & 110 \\
\hline MARY ELLEN SHOP - women's clothes & 357 & MAIN STREET & 562 & M & 85 \\
\hline LADY FAIR SHOPPE - women's clothes & 205 & E. LAKE ST & 562 & $\mathbf{M}$ & 71 \\
\hline OLANDER'S - children's clothes & 433 & MAIN STREET & 564 & M & 784 \\
\hline WATSONVILLE TOYLAND & 135 & E. LAKE ST & 564 & M & 731 \\
\hline WATSONVILLE OUTLET - genl mdse ** & 275 & MAIN STREET & 565 & $M$ & 260 \\
\hline G.B. FAMILY WEAR & 311 & MAIN STREET & 565 & $M$ & 164 \\
\hline JOHN'S SHOE STORE & 434 & MAIN STREET & 566 & M & 304 \\
\hline VAN'S SHOES + REPAIR & 12 & E. LAKE ST & 566 & M & 298 \\
\hline \multirow{2}{*}{$\begin{array}{l}\text { KARL'S SHOE STORE } \\
\text { FORBES SHOES }\end{array}$} & 339 & MAIN STREET & 566 & M & 254 \\
\hline & 425 & MAIN STREET & 566 & $\mathbf{M}$ & 209 \\
\hline MCMAHAN FURN & 543 & MAIN STREET & 571 & M & 1607 \\
\hline SWEENEY'S USED FURN & $* * 242$ & MAIN STREET & 571 & M & 549 \\
\hline HOFFMAN INTERIORS - furniture & 23 & E. LAKE ST & 571 & M & 450 \\
\hline \multirow{2}{*}{$\begin{array}{l}\text { DEWORKEN FURN } \infty \\
\text { UNION FURNTURE } \infty\end{array}$} & 19 & E. LAKE ST & 571 & M & 339 \\
\hline & 55 & BRENNAN ST & 571 & M & 275 \\
\hline GOOFRICH TYPEWRTERS + OFFICE SUPP & $P 440$ & MAIN STREET & 572 & M & 235 \\
\hline SINGER SEWING MACH CO & 430 & MAIN STREET & 572 & M & 218 \\
\hline $\begin{array}{l}\text { HUNTING BYE REFGR + SUP CO. } \\
\text { DEE'S SEWING MACHINE + VAC CENTER }\end{array}$ & 115 & E. LAKE ST & 572 & M & 150 \\
\hline DEE'S SEWING MACHINE + VAC CENTER & 510 & MAIN STREET & 572 & 1 & 139 \\
\hline JANSEN'S MUSIC RADIO & 435 & MAIN STREET & 573 & M & 392 \\
\hline ROYAL GRILL - rest & 418 & MAIN STREET & 581 & M & 467 \\
\hline $\begin{array}{l}\text { WING'S CAFE - rest } \\
\text { MIRAMAR GRILL }\end{array}$ & 178 & MAIN STREET & 581 & M & 322 \\
\hline \multirow{2}{*}{$\begin{array}{l}\text { MIRAMAR GRILL } \\
\text { NOOK CAFE - rest }\end{array}$} & 526 & MAIN STREET & 581 & M & 247 \\
\hline & 550 & TREET & 581 & M & 207 \\
\hline EL MICHOACAN - rest & 272 & MAIN STREET & 581 & M & 194 \\
\hline PETER PAN CAFE - rest & 621 & MAIN STREET & 581 & M & 194 \\
\hline STAR CAFE & 229 & MAIN STREET & 581 & M & 163 \\
\hline MORELIA CAFE & 256 & MAIN STREET & 581 & $M$ & 163 \\
\hline RESETAR HARVEST ROOM - rest & 15 & W. LAKE ST & 581 & M & 163 \\
\hline LA POBLANITA CAFE & 180.5 & STREET & 581 & M & 152 \\
\hline FOSTER'S - rest & 652 & MAIN STREET & 581 & $M$ & 132 \\
\hline GOLDENEAGLE CAFE & 263 & MAIN STREET & 581 & $M$ & 115 \\
\hline YMCA SALVAGE STORE & 302 & UNION STREET & 593 & M & 581 \\
\hline RED FRONT SURPLUS & 227 & MAIN STREET & 593 & M & 260 \\
\hline GOODWILL - used goods & 22 & E. LAKE ST & 59 & N & 96 \\
\hline JOHNSON STATIONERY & 341 & TREET & 594 & $M$ & 214 \\
\hline VALLEY SPORT SHOP & 202 & E. LAKE ST & 595 & $M$ & 337 \\
\hline SHARP'S SPORTING GOODS & 536 & MAIN STREET & 595 & M & 143 \\
\hline ALDOUS + HOFER JWLR & 323 & MAIN STREET & 597 & $M$ & 320 \\
\hline DANZZKER JWLRY AND LOAN CO & 252 & MAIN STREET & 597 & $\mathbf{M}$ & 260 \\
\hline JOLLEY'S JWLRS & 442 & MAIN STREET & 597 & M & 169 \\
\hline MORRIE'S CREDIT JWLRS & 316 & MAIN STREET & 597 & M & 78 \\
\hline YARDAGE SHOP - material & 426 & MAIN STREET & 599 & $M$ & 218 \\
\hline FLOWER GIFT SHOP & 531 & MAIN STREET & 599 & M & 239 \\
\hline JENKEL DAVIDSON OPTICAL GOODS & 629 & MAIN STREET & 599 & M & 66 \\
\hline PAJARO VALLEY BANK & 326 & MAIN STREET & 611 & M & 640 \\
\hline The & 00 & $\begin{array}{l}\text { MAIN STREET } \\
89\end{array}$ & 611 & M & 201 \\
\hline
\end{tabular}




\begin{tabular}{|c|c|c|c|c|c|}
\hline MIEDL REALTY CO & 424 & MAIN STREET & 615 & M & 92 \\
\hline STOUT REALTY CO & 632 & MAIN STREET & 615 & M & 92 \\
\hline BOSWORTHREAL ESTATE & 5 & E. LAKE ST & 615 & M & 72 \\
\hline CONSOLE + KRATZ REAL ESTATE & 629 & MAIN STREET & 615 & $\mathbf{M}$ & 66 \\
\hline KANE REAL ESTATE & $30 \mathrm{~A}$ & E.5TH STREET & 615 & $M$ & 65 \\
\hline WATSONVIULEPROPERTIES & $30 \mathrm{~B}$ & E. 5TH STREET & 615 & M & 65 \\
\hline ANDERSON REAL ESTATE & 30.5 & E. 5TH STREET & 615 & M & 65 \\
\hline HALL + SON REAL ESTATE & 129 & E. LAKE ST & 615 & $M$ & 16 \\
\hline FOXMOVIE THEATER & 304 & MAIN STREET & 621 & $\mathrm{M}$ : & 224 \\
\hline WHITE'S MEMORIAL CHAPEL & 609 & MAIN STREET & 624 & $\mathbf{M}$ & 641 \\
\hline ZAR MOTORS BODY SHOP & 643 & MAIN STREET & 641 & $M$ & 374 \\
\hline WILLIE'S SHOE SHOP - reprS & 109 & UNION STREET & 641 & $M$ & 284 \\
\hline YOUNG'S INSIDE GARAGE & 440.5 & MAIN STREET & 641 & M & 235 \\
\hline HOUSEHOLD APPL SERV & 544 & MAIN STREET & 649 & $\mathrm{M}$ & 260 \\
\hline FRANK'S RADIO SERV & 107 & UNION STREET & 649 & M & 115 \\
\hline COOTS ART RADIO SHOP & 267 & MAIN STREET & 649 & M & 63 \\
\hline LINDGRENRADIO SPEED SERVICE & 40 & W. LAKE ST & 649 & M & 51 \\
\hline AMIZICH BROS PLUMBSINSULATION & 117 & E. LAKE ST & 662 & M & 153 \\
\hline CENTRE THEATRE & 223 & MAIN STREET & 721 & $\mathrm{M}$ & 518 \\
\hline NUMBER OFM & & 112 & & & \\
\hline TOTAL M2 OFM & & 35925 & & & \\
\hline AVE M2 OF M & & 321 & & & \\
\hline$\%$ OF M (AREA) & & 64.5 & & & \\
\hline SDFORM & & 304 & & & \\
\hline
\end{tabular}

*** Establishment assigned approximate value for area 
TABLE 26 : MASS APPEAL STORES IN WATSONVILLE IN 1988 BY STANDARD LAND USE CODE

\begin{tabular}{|c|c|c|c|c|c|}
\hline Name of Establishement & Street & Address & $\begin{array}{l}\text { Standard } \\
\text { Land Use } \\
\text { Code }\end{array}$ & Type & Area $\mathrm{M}$ \\
\hline YELLOW CAB CO. & 30 & E. 5TH STREET & 429 & $M$ & 65 \\
\hline BOB GROSS TRAVEL & 343 & RODRIGUEZST & 492 & $M$ & 161 \\
\hline GREENE TRAVEL SERVICE & 21.6 & BRENNAN ST & 492 & M & 110 \\
\hline WATSONVILLE PAINT CENTER & 548.5 & MAIN STREET & 523 & M & 125 \\
\hline HARRISON'S COLOR CORNER - paint & 143 & E. LAKE ST & 523 & $M$ & 67 \\
\hline ACE HARDWARE & 317 & MAIN STREET & 525 & M & 594 \\
\hline FORD'S - dept store & 407 & MAIN STREET & 531 & M & 3268 \\
\hline J.C. PENNY - dept store & 501 & MAIN STREET & 531 & $M$ & 1348 \\
\hline WOOLWORTH'S & 451 & MAIN STREET & 531 & M & 1060 \\
\hline FREEDOMFOOD CENTER & 133 & E. LAKE ST & 541 & $\mathrm{Mi}$ & 920 \\
\hline FLOR DEL VAUE MARKET & 100 & UNION STREET & 542 & $\mathbf{M}$ & 790 \\
\hline WATSONVILLE LOCKERS - meat & 625 & MAIN STREET & 542 & M & 200 \\
\hline BAKERY THRIFT STORE and storage & 330 & RODRIGUEZST & 546 & $\mathbf{M}$ & 572 \\
\hline BAKE-RITE BAKERY & 21 & E. BEACH ST & 546 & $\mathbf{M}$ & 261 \\
\hline MAIN STREET BAKERY & 521 & MAIN STREET & 546 & M & 47 \\
\hline GRAND AUTO & 401 & RODRIGUEZST & 552 & M & 620 \\
\hline WATSONVILLE TIRE SERVICE & 27 & FIRST STREET & 552 & M & 382 \\
\hline SWEET SERVICE CO. - auto parts & 480 & UNION STREET & 552 & $\mathbf{M}$ & 372 \\
\hline TOWNSEND'S AUTO PARTS & 307 & RODRIGUEZST & 552 & M & 353 \\
\hline WESTERN AUTO & 439 & MAIN STREET & 552 & M & 278 \\
\hline WATSONVILLE JEANS & 347 & MAIN STREET & 561 & M & 681 \\
\hline DICK BRUHN MEN'S STORE & 347 & RODRIGUEZST & 561 & $\mathbf{M}$ & 653 \\
\hline CALIFORNIA FASION WEAR FOR MEN & 437 & MAIN STREET & 561 & $\mathbf{M}$ & 465 \\
\hline LAZY DAY - men's cloths & 428 & MAIN STREET & 561 & M & 218 \\
\hline HELEN'S CORNER - clothes & 531 & MAIN STREET & 562 & M & 239 \\
\hline ERNIE'S LADY FAIRE SHOPPE & 441 & MAIN STREET & 562 & M & 109 \\
\hline LYDIA'S CHILDREN'S WEAR & 463 & MAIN STREET & 564 & M & 40 \\
\hline FAMILY BARGAIN CENTER - clothes & 333 & MAIN STREET & 565 & M & 836 \\
\hline WATSONVILLE FASIONS & 426 & MAIN STREET & 565 & $M$ & 218 \\
\hline G.B. FAMILY WEAR & 311 & MAAIN STREET & 565 & M & 164 \\
\hline CASA ORTIZ - clothes & 257 & MAIN STREET & 565 & M & 63 \\
\hline JOHN'S SHOE STORE & 456 & MAIN STREET & 566 & M & 751 \\
\hline ZAPATERIA HERMANOS - shoes & 345 & TREET & 566 & $\mathbf{M}$ & 329 \\
\hline CANADA SHOES & 301 & TREET & 566 & M & 328 \\
\hline VAN'S SHOES + REPAIR & 14 & E. LAKE ST & 566 & M & 298 \\
\hline MCMAHAN'S FURNITURE & 553 & MAIN STREET & 571 & $M$ & 1607 \\
\hline CARPET HOUSE & 516 & MAIN STREET & 571 & M & 461 \\
\hline FURNITURE & 19 & E. LAKE ST & 571 & M & 339 \\
\hline BURDICK'S APPLIANCES + TV & 433 & MAIN STREET & 572 & $M$ & 784 \\
\hline BAKER BROS. FURNITURE - appl.+video & 55 & BRENNAN ST & 572 & M & 275 \\
\hline MAYTAG APPLIANCES & 550 & MAIN STREET & 572 & $M$ & 207 \\
\hline DERRICK'S MAYTAG & 555 & MAIN STREET & 572 & M & 207 \\
\hline SINGER + KIRBY VAC. + SEWING & 445 & MAIN STREET & 572 & $M$ & 109 \\
\hline HUNTING'S REFRIGERATOR + AIR COND. & 119 & E. LAKE ST & 572 & $M$ & 106 \\
\hline
\end{tabular}




\begin{tabular}{|c|c|c|c|c|c|}
\hline RADIOS & 314 & MAIN STREET & 573 & M & 70 \\
\hline LEJANE'S - rest & 418 & MAIN STREET & 581 & $M$ & 467 \\
\hline RICO POLLO - rest & 353 & MAIN STREET & 581 & $M$ & 334 \\
\hline JALISCO - rest & 323 & MAIN STREET & 581 & $M$ & 320 \\
\hline LA FOGATA - rest & 109 & UNION STREET & 581 & M & 284 \\
\hline PHILIPPINE GARDENS - rest & 26 & W. BEACH ST & 581 & $M$ & 175 \\
\hline RUBEN'S - rest & 458 & MAIN STREET & 581 & $M$ & 166 \\
\hline DAIRY QUEEN & 130 & E. LAKE ST & 581 & M & 148 \\
\hline KINGS GARDEN - rest & 412 & MAIN STREET & 581 & M & 148 \\
\hline CHOPSTICK - rest & 508 & MAIN STREET & 581 & $M$ & 139 \\
\hline PIZZAMIA & 34 & W. BEACH ST & 581 & $M$ & 133 \\
\hline SUPERBURITO & 652 & MAIN STREET & 581 & M & 132 \\
\hline CLUB MIRAMAR - bar & 522 & MAIN STREET & 581 & M & 130 \\
\hline MIRAMAR GRILL & 526 & MAIN STREET & 581 & $\mathbf{M}$ & 130 \\
\hline EL POLLERO - rest & 168 & MAIN STREET & 581 & $M$ & 117 \\
\hline MIRAMAR BANQUET ROOM & 526 & MAIN STREET & 581 & $\mathrm{M}$ & 117 \\
\hline RESTAURANTE JALISCO & 263 & MAIN STREET & 581 & $M$ & 115 \\
\hline NOOKCAFE & 106 & E. LAKE ST & 581 & $M$ & 107 \\
\hline EL FRIJOLITO - rest & $11 \mathrm{~B}$ & ALEXANDER ST & 581 & $M$ & 92 \\
\hline TACOBUENO & 720 & MAIN STREET & 581 & M & 91 \\
\hline EXPRESSO ICE CREAM AND COFFEE & $521 \mathrm{~A}$ & MAIN STREET & 581 & M & 75 \\
\hline MORELLA MEXICAN FOOD & 312 & MAIN STREET & 581 & M & 70 \\
\hline LA FONDITA - rest & 30 & E. 5TH STREET & 581 & $M$ & 65 \\
\hline LA MAZANA DELI & 521 & MAIN STREET & 581 & $M$ & 47 \\
\hline PLAYITA DEL SOL - rest & 315 & MAIN STREET & 581 & M & 45 \\
\hline LA FIESTA - rest & 9 & W. BEACH ST & 581 & $M$ & 36 \\
\hline RESTUARANTE PLAYAS NOVIELLERO & 251 & MAIN STREET & 582 & M & 215 \\
\hline PHARMACIA INTERNATIONAL & 325 & MAIN STREET & 591 & M & 230 \\
\hline GOODWILL - thrift shop & 470 & MAIN STREET & 593 & M & 897 \\
\hline ST. VINCENT DE PAUL THRIFT STORE & 135 & E. LAKE ST & 593 & M & 731 \\
\hline SALVATION ARMY THRIFT SHOP & 23 & E. LAKE ST & 593 & M & 450 \\
\hline TAYLORS OFFICE SUPPLIES & 436 & MAIN STREET & 594 & M & 471 \\
\hline POOR RICHARD'S ALMANAC BUSINESS & 25 & E. 5TH STREET & 594 & $\mathbf{M}$ & 440 \\
\hline FELIXS RECORD SHOP + VIDEO & 327 & MAIN STREET & 594 & M & 170 \\
\hline DISCOTECA MEXICO - records & 261 & MAIN STREET & 594 & M & 115 \\
\hline ROMANE JEWLERS & 471 & MAIN STREET & 594 & $M$ & 110 \\
\hline WATSONVILLE CYCLERY & 202 & E. LAKE ST & 595 & M & 337 \\
\hline MORELIA SPORTS SHOP & 517 & MAIN STREET & 595 & M & 47 \\
\hline DON ROBERTO JEWLERS & 339 & MAIN STREET & 597 & $M$ & 254 \\
\hline JOLEY'S JEWLERY & 442 & MAIN STREET & 597 & $M$ & 169 \\
\hline GONZALES JEWLERS & 316 & MAIN STREET & 597 & $M$ & 78 \\
\hline PAKMAIL & 434 & MAIN STREET & 599 & M & 304 \\
\hline FLORIST & 5 & E. LAKE ST & 599 & M & 72 \\
\hline FREDDYS ONE HOURPHOTO & 450 & MAIN STREET & 599 & M & 130 \\
\hline GREAT WESTERN BANK & 630 & MAIN STREET & 611 & M & 992 \\
\hline SUMITOMO BANK & 558 & MAIN STREET & 611 & $M$ & 749 \\
\hline WELLSFARGO BANK & 326 & MAIN STREET & 611 & M & 640 \\
\hline WATSONVILLEE FEDERAL SAVINGS & 35 & E. LAKE ST & 611 & M & 613 \\
\hline BANK OF AMERICA & 567 & $\begin{array}{l}\text { MAIN STREET } \\
9 ?\end{array}$ & 611 & M & 610 \\
\hline
\end{tabular}




\begin{tabular}{|c|c|c|c|c|c|}
\hline HOUSEHOLDBANK & 318 & UNION STREET & 611 & $M$ & 366 \\
\hline MONTEREY SAVINGS & 375 & MAIN STREET & 611 & M & 256 \\
\hline PACIFIC WESTERN BANK & 400 & MAIN STREET & 611 & $\mathbf{M}$ & 201 \\
\hline PAJARO VALLEY BANK & 655 & MAIN STREET & 611 & M & 90 \\
\hline BURGSTROMKRAMER REALTORS & 21.16 & BRENNAN ST & 615 & $M$ & 110 \\
\hline NETWORK REALESTATE & 600 & MAIN STREET & 615 & $M$ & 70 \\
\hline HANSEN PROPERTIES & 629 & MAIN STREET & 615 & $M$ & 66 \\
\hline WATSONVILLE PROPERTIES & $30 \mathrm{~B}$ & E. 5TH STREET & 615 & $M$ & 65 \\
\hline QUINTERO REALTORS & 5 & CARR STREET & 615 & $M$ & 53 \\
\hline HALWARD REALTORS & 40 & W. LAKE ST & 615 & M & 51 \\
\hline DAVIS MEMORIAL CHAPEL - funeral h. & 609 & MAIN STREET & 624 & $M$ & 641 \\
\hline QUALITY, AUTO, BODY REPAIR SHOP & 335 & RODRIGUEZST & 641 & $M$ & 1116 \\
\hline GOLDEN STATE AIR + RADIATOR & 469 & UNION STREET & 641 & $M$ & 518 \\
\hline AAMCO & 640 & MAIN STREET & 641 & $M$ & 494 \\
\hline DON FISHER MOTORS - repair & 639 & MAIN STREET & 641 & $M$ & 327 \\
\hline KAY'S GARAGE & 259 & RODRIGUEZST & 641 & $M$ & 174 \\
\hline H + S SERVICE GARAGE & 25 & FIRST STREET & 641 & $M$ & 129 \\
\hline PANARO SHOW PLACE & 425 & UNION STREET & 721 & M & 637 \\
\hline FOX MOVIE THEATER & 304 & MAIN STREET & 721 & $M$ & 224 \\
\hline NUMBER OFM & & 113 & & & \\
\hline TOTAL M2 OF M & & 39261 & & & \\
\hline AVE M2 OF M & & 347 & & & \\
\hline$\%$ OF M (AREA) & & 75.3 & & & \\
\hline SDFORM & & 409 & & & \\
\hline
\end{tabular}




\section{HOLLISTER}

\section{$1960(1961)-1988$}

Hollister is located southwest of Gilroy and south of San Jose. The downtown extends for eight blocks along San Benito Street and perpendicular to it for about a block on both sides. (see map 19) Almost all of the retail stores in Hollister are included in the downtown area. (see table 2) The central business district is bounded on the east by the industrial zone, and on the north and west by residential areas. To the south there is farmland and highway -- with a little "non-downtown" retail space. Along this highway, a growth in retail is underway, including small shopping malls and restaurant chain stores.

The population of Hollister in 1960 was only 6070 , making it the smallest city in the study. Almost all of the retail stores were in the downtown retail area. (see table 2) There were 38,335 square meters of retail space in Hollister's CBD. Almost all of the retail stores in Hollister, $96 \%$, were in the CBD. The average size of the stores changes significantly with type. As one would expect, the convenience stores tended to be the smallest (ave $156 \mathrm{~m}^{2}$ ); the limited appeal stores were in the middle (ave $264 \mathrm{~m}^{2}$ ), and the mass appeal stores, which include the large department stores, were the largest (ave $368 \mathrm{~m}^{2}$ ). The high standard deviations, however, make size an unreliable indicator. For all types of stores, the average size was slightly smaller for the "main street" (San Benito Street). This would be expected because rents tend to be higher on the "main street." 


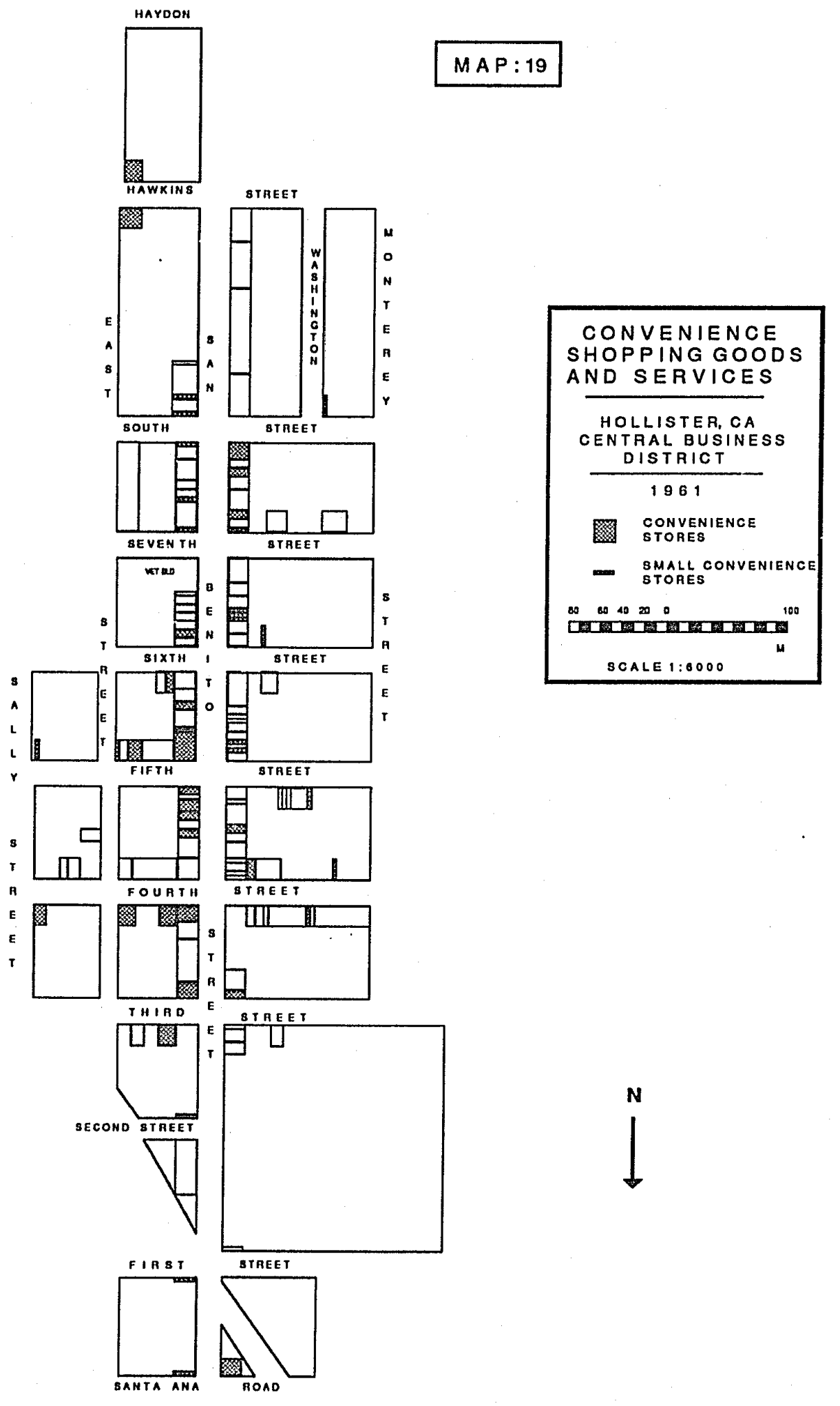


The population of Hollister from 1960 to 1987 increased by $177 \%$ to 16,800 people. This large change in population was accompanied by only a small increase in the retail area of the central business district: $7.6 \%$. This inequality caused a significant decrease in the amount of vacant space since the increase in population was not accompanied by much construction of retail buildings. There has also been a recent increase in the amount of retail space outside of the downtown.

\section{Convenience Space}

More than a fifth of the total retail space in Hollister's central business district (CBD) was convenience. (see fig 4) Of the 42 possible corner store locations, one half of them were convenience type stores. The percentage of convenience stores per block ranges from $0 \%$ to $100 \%$. The blocks with all convenience stores have very little total retail space and tend to fall on the edge of the downtown area. (see map 19) The large percentage of convenience stores and their distribution throughout the downtown area indicate the multifunctionality of Hollister's downtown.

Convenience stores from 1961 to 1988 decreased in the percentage of the total retail space used by about a third. (see fig 9) The decrease in convenience space was fairly uniform throughout the downtown, with only a slightly larger perceniage eliminated from San Benito Street. (see map 20) But the decrease in convenience space was not uniform for all types of convenience stores. In 1961, 15.9\% of convenience space consisted of corner markets. By 1988, the percentage 
Fig. 4

Percentages of retail land use in the CBD HOLLISTER

HOLLISTER 1961

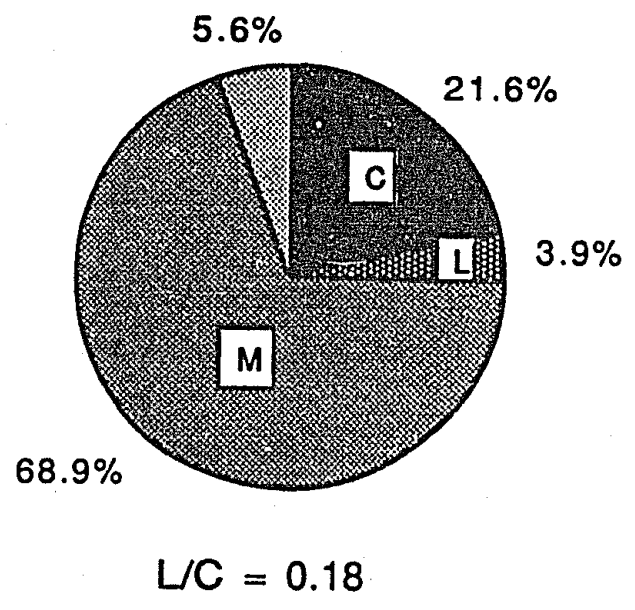

HOLLISTER 1988

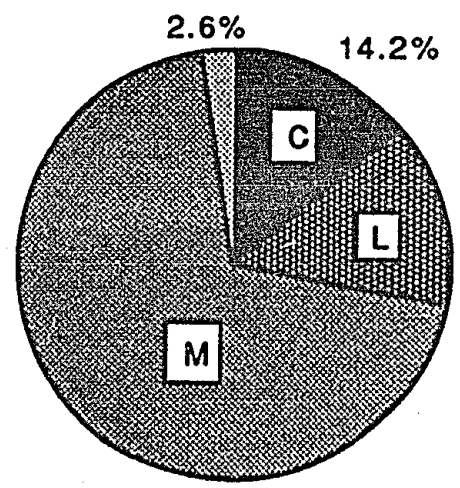

$69.4 \%$

$$
L / C=0.97
$$

HOLLISTER 1961

THE MAIN ST ONLY

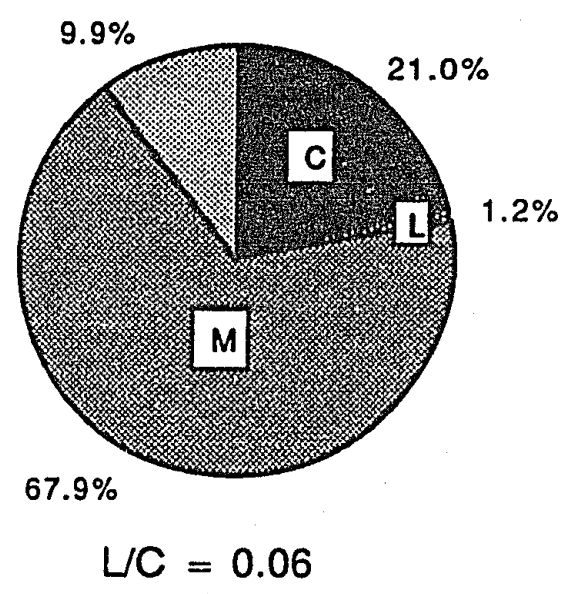

HOLLISTER 1988

THE MAIN ST ONLY

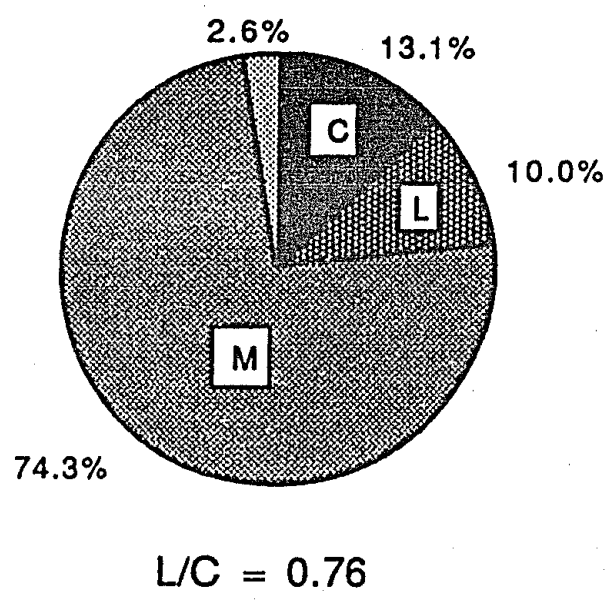




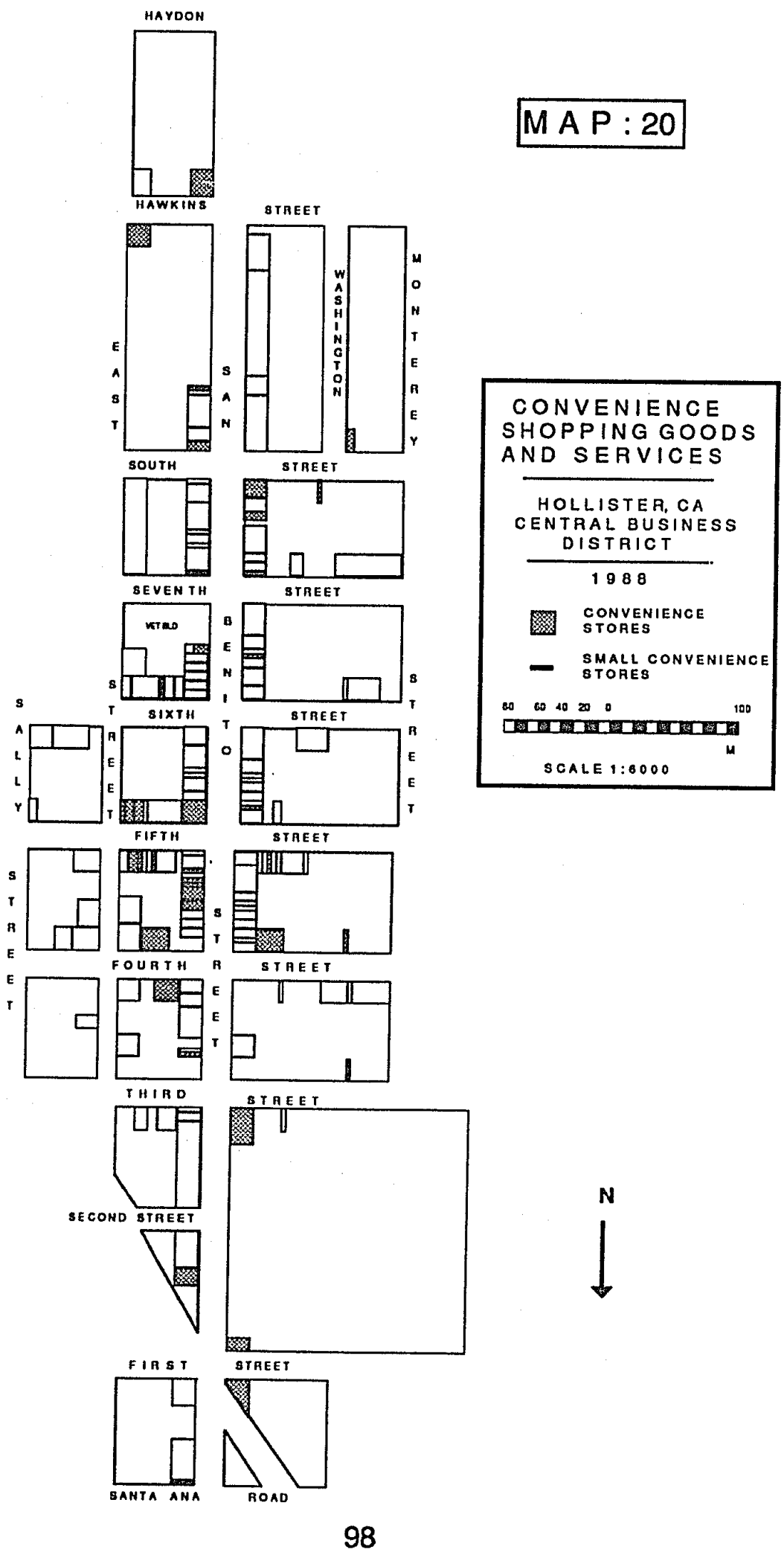


decreased to only $7.8 \%$. Gas stations decreased even more dramatically from $10 \%$ to $2 \%$. Convenience restaurants and bars also decreased significantly. Beauty salons and barber shops however, increased from $12 \%$ to $20 \%$. Video rental stores, which did not exist in 1961 , now account for $18 \%$ of the convenience space. Liquor stores and drug stores have remained fairly constant. (see tables 27 and 28 )

\section{Limited Appeal Space}

Only $5.5 \%$ of the retail area however, was limited appeal. (see map 21) On San Benito Street it was a mere 1.2\%. The large lumber store on Seventh Street accounts for much of the total. The only other limited appeal stores are: Western Union telegraph, a glass store, a vending machine store, a health food grocery, a saddle shop, and a photography studio. (see tables 29 and 30 ) The ratio of limited appeal stores to convenience stores is only .18 for the downtown as a whole and a mere .06 for the main street. (see fig. 4)

By 1988 , the percent of retail space in limited appeal goods more than tripled to $13.8 \%$. The ratio of limited appeal space to convenience space grew from .18 to .97 , i.e. to an almost equal amount of each. (see map 22)

Mass Appeal Space (maps 23 and 24)

The mass appeal stores, for almost any downtown, account for the vast majority of the retail space. In $1961,68.9 \%$ of the total downtown retail floorspace was taken up by mass appeal stores. (see fig. 
TABLE 27 : CONVENIENCE STORES IN HOLLISTER IN 1961 BY STANDARD LAND USE CODE

\begin{tabular}{|c|c|c|c|c|c|c|}
\hline \multicolumn{2}{|l|}{ Name of Establishement } & \multicolumn{2}{|c|}{ Street Address } & \multicolumn{3}{|c|}{$\begin{array}{l}\text { Standard } \\
\text { Land Use } \\
\text { Code }\end{array}$} \\
\hline \multirow{2}{*}{\multicolumn{2}{|c|}{$\begin{array}{l}\text { CAPUTOS MARKET - grocery } \\
\text { PUB DRIVE-IN MARKET }\end{array}$}} & 203 & HAWKINS ST & 541 & C & 312 \\
\hline & & 756 & SAN BENITO ST & 541 & C & 267 \\
\hline \multirow{2}{*}{\multicolumn{2}{|c|}{$\begin{array}{l}\text { SAVE-MORE-MKT - grocery } \\
\text { MISSION FOOD MARKET }\end{array}$}} & 355 & SAN BENITO ST & 541 & C & 236 \\
\hline & & 307 & SAN BENITO ST & 541 & C & 232 \\
\hline \multicolumn{2}{|l|}{ GUILHAMETS PALACE MARKET } & 728 & SAN BENITO ST & 541 & C & 150 \\
\hline \multicolumn{2}{|l|}{ GRAHAM'S MARKET } & 636 & SAN BENITO ST & 541 & C & 115 \\
\hline FARM MILK-DRIVE IN & *** & 4 & SAN BENITO ST & 545 & $\mathrm{C}$ & 232 \\
\hline \multicolumn{2}{|l|}{ LANGDON GAS STATION } & 140 & 4TH STREET & 553 & C & 302 \\
\hline \multirow{2}{*}{\multicolumn{2}{|c|}{$\begin{array}{l}\text { LAWRENCE GAS STATION } \\
\text { CONLEY'S TEXACO SERVICE STATION }\end{array}$}} & 5 & SAN BENITO ST & 553 & C & 167 \\
\hline & & 249 & SAN BENITO ST & 553 & C & 93 \\
\hline \multirow{2}{*}{\multicolumn{2}{|c|}{$\begin{array}{l}\text { CHEVRON GAS STATION } \\
\text { NICK'S GAS STATION }\end{array}$}} & 395 & 4TH STREET & 553 & c & 76 \\
\hline & & 821 & SAN BENITO ST & 553 & C & 75 \\
\hline \multicolumn{2}{|l|}{ BILL'S SERVICE - gas station } & 125 & SAN BENITO ST & 553 & C & 52 \\
\hline \multicolumn{2}{|l|}{ UNION GAS STATION } & 102 & BENITO ST & 553 & C & 37 \\
\hline \multirow{2}{*}{\multicolumn{2}{|c|}{$\begin{array}{l}\text { PETREE SHELL GAS STATION } \\
\text { UNION GAS STATION }\end{array}$}} & 763 & SAN BENITO ST & 553 & C & 26 \\
\hline & & 710 & SAN BENITO ST & 553 & C & 6 \\
\hline \multicolumn{2}{|l|}{ NICKS LUNCH REST } & 801 & SAN BENITO ST & 581 & C & 200 \\
\hline VILLA PACE REST \& BAR & $m * *$ & 202 & 4THSTREET & 581 & C & 194 \\
\hline \multicolumn{2}{|l|}{ F \& F DONUT SHOP } & 510 & SAN BENITO ST & 581 & C & 112 \\
\hline \multicolumn{2}{|l|}{ DING-A-LING CAFE } & 380 & 4TH STREET & 581 & C & 80 \\
\hline \multicolumn{2}{|l|}{ BRAND CLUB - bar } & 219 & SOUTH STREET & 582 & c & 215 \\
\hline \multicolumn{2}{|l|}{ SAN BENITO AND TAVERN } & 220 & STREET & 582 & C & 176 \\
\hline \multicolumn{2}{|l|}{ KING PIN COCKTAIL LOUNGE } & 304 & OST & 582 & C & 170 \\
\hline \multicolumn{2}{|l|}{ SMOKE HOUSE - bar } & 427 & SAN BENITO ST & 582 & C & 165 \\
\hline \multicolumn{2}{|l|}{ ACME CLUB - bar } & 419 & SAN BENITO ST & 582 & C & 141 \\
\hline \multicolumn{2}{|l|}{ JOHNNIE'S BAR } & 525 & SAN BENITO ST & 582 & c & 125 \\
\hline \multicolumn{2}{|l|}{ PAINES CLUB - bar } & 202 & 5TH STREET & 582 & $\mathrm{C}$ & 120 \\
\hline \multicolumn{2}{|l|}{ RUSTIC GARDENS - bar } & 321 & 4TH STREET & 582 & C & 101 \\
\hline VICTORIA CLUB - bar & & 201 & STREET & 582 & C & 83 \\
\hline WHALES & & 501 & BENITO ST & 591 & C & 461 \\
\hline PHARM & "** & 434 & SAN BENITO ST & 591 & C & 222 \\
\hline PHARM & & 520 & BENITO ST & 591 & C & 195 \\
\hline AICHELE'S LIQUOR STORE & *** & 203 & STREET & 592 & C & 289 \\
\hline DABO LIQUORS & *** & 220 & 6TH STREET & 592 & c & 289 \\
\hline VIEIRA'S LIQUORS & & 898 & SAN BENITO ST & 592 & C & 182 \\
\hline J AND L LIQUOR STORE & & 417 & SAN BENITO ST & 592 & C & 141 \\
\hline WASH AND DRY - laundry & & 204 & HAWKINS ST & 621 & C & 293 \\
\hline UNONFRENCH LNDRY & & 216 & 3AD STREET & 621 & C & 236 \\
\hline MODEL FRENCH LAUNDRY & $* *$ & 127 & 5TH STREET & 621 & C & 139 \\
\hline SAN BENITO LAUNDROMAT & $* * *$ & 59 & SAN BENITO ST & 621 & C & 139 \\
\hline STANLEY CLEANERS & & 713 & SAN BENITO ST & 621 & C & 111 \\
\hline FASHION CLEANERS & & 611 & SAN BENITO ST & 621 & C & 102 \\
\hline SQUARE DEAL BARBER Sト & & 215 & 5TH STREET & 623 & C & 319 \\
\hline
\end{tabular}




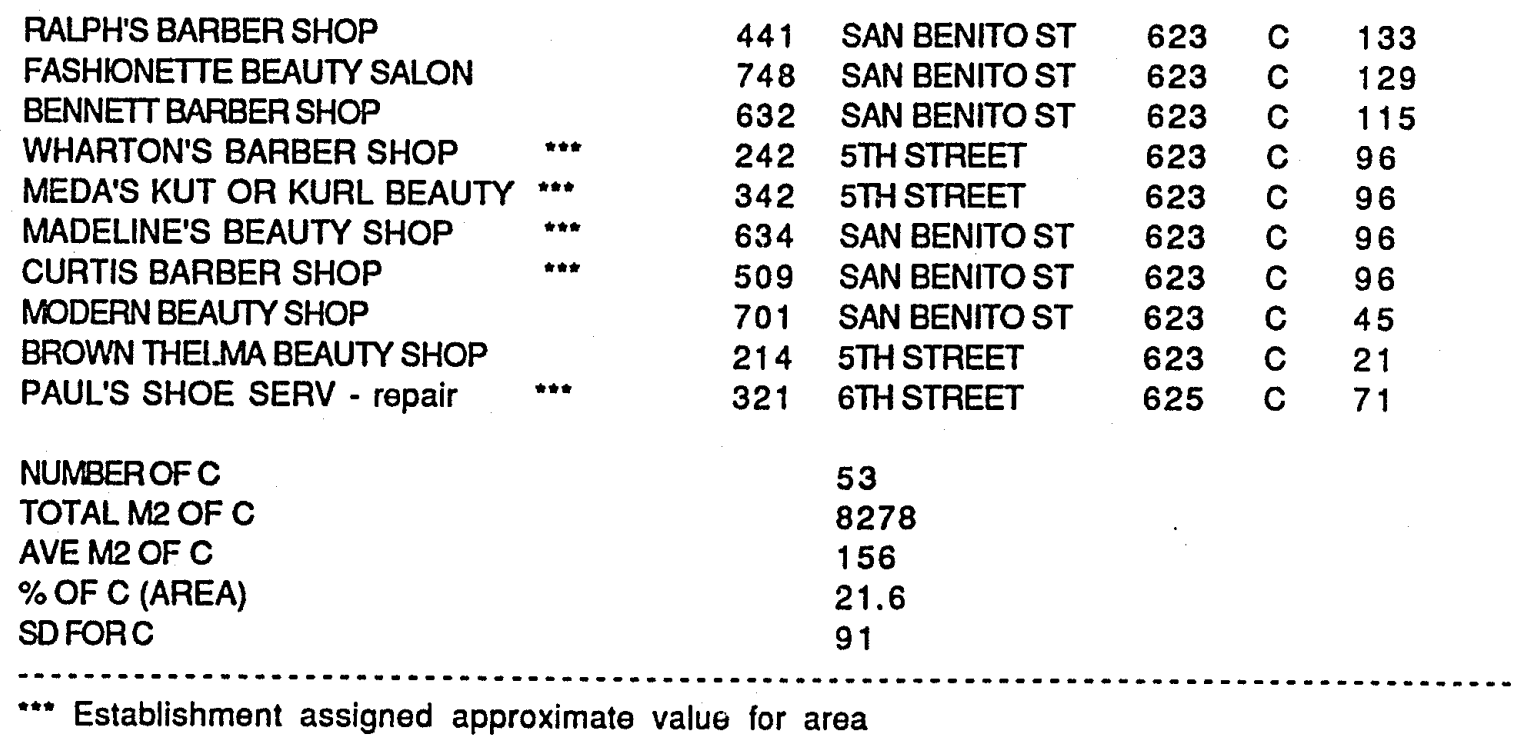


TABLE 28: CONVENIENCE STORES IN HOLLISTER IN 1988 BY STANDARD LAND USE CODE

\begin{tabular}{|c|c|c|c|c|c|}
\hline Name of Establishement & Stree & Address & $\begin{array}{l}\text { andard } \\
\text { nd Use } \\
\text { de }\end{array}$ & ype & Area $M^{2}$ \\
\hline BROTHERS MARKET & 756 & SAN BENITO ST & 541 & C & 267 \\
\hline SEVEN-ELEVEN & 915 & SAN BENITO ST & 541 & C & 192 \\
\hline BEACON - gas and auto repair & 250 & SAN BENITO ST & 553 & C & 60 \\
\hline 76 - gas and auto repair & 102 & SAN BENITO ST & 553 & C & 37 \\
\hline CHEVRON - gas & 375 & 4TH STREET & 553 & C & 21 \\
\hline EXXON - gas & 5 & SAN BENITO ST & 553 & C & 13 \\
\hline MOBIL - gas & 710 & SAN BENITO ST & 553 & C & 6 \\
\hline BJ'S CHILI DOGS & 125 & SÃN BENITO ST & 581 & C & 18 \\
\hline BRAND CLUB - bar & 219 & SOUTH STREET & 582 & C & 215 \\
\hline SILVER BULLET - bar & 220 & 4TH STREET & 582 & c & 176 \\
\hline SMOKE HOUSE - bar & 427 & SAN BENITO ST & 582 & C & 165 \\
\hline OFFICE, THE - bar & 321 & 4TH STREET & 582 & C & 101 \\
\hline WHISKY CAEEK SALOON & 201 & 5TH STREET & 582 & C & 83 \\
\hline PENNY WISE DRUG & 549 & SAN BENITO ST & 591 & C & 465 \\
\hline WHALERDRUGS & 501 & SAN BENITO ST & 591 & C & 461 \\
\hline LYNN'S LIQUORS & 204 & HAWKINS ST & 592 & C & 293 \\
\hline RIGHETTILIQUORS & 310 & FIRST STREET & 592 & C & 293 \\
\hline DUBOLLUORS & 801 & SAN BENITO ST & 592 & C & 275 \\
\hline HOUSTER VIDEO & 217 & 4TH STREET & 592 & C & 420 \\
\hline AMERICAN VIDEO MOVIE RENTAL & 211 & 5TH STREET & 592 & C & 269 \\
\hline VIDEO & 435 & SAN BENITO ST & 592 & C & 237 \\
\hline BOB'S VIDEO & 510 & SAN BENITO ST & 592 & C & 112 \\
\hline PATTI'S MINI FLORIST & 443 & SAN BENITO ST & 599 & C & 55 \\
\hline PHOTO CORNER & 701 & SAN BENITO ST & 599 & C & 45 \\
\hline COLONIAL CLEANERS & 355 & SOUTH STREET & 621 & C & 110 \\
\hline GRANNY'S WASH + DRY & 231 & 3RD STREET & 621 & C & 101 \\
\hline MARTINIZING DRY CLEANERS & 231 & 3RD STREET & 621 & C & 101 \\
\hline B + R BARBERSHOP & 215 & 5TH STREET & 623 & C & 208 \\
\hline CALIFORNIA HAIR CLUB & 853 & SAN BENITO ST & 623 & C & 174 \\
\hline HAIRELITE & 206 & 5TH STREET & 623 & c & 139 \\
\hline BEAUTY DEN & 748 & SAN BENITO ST & 623 & $\mathrm{C}$ & 129 \\
\hline BIL'S BARBER SHOP & 632 & SAN BENITO ST & 623 & C & 115 \\
\hline JOHNNIES BARBER SHOP & 326 & 5TH STREET & 623 & C & 96 \\
\hline NANCY'S BEAUTY SALON & 625 & SAN BENITO ST & 623 & C & 93 \\
\hline NEW IMAGE - hair and tanning & 437 & SAN BENITO ST & 623 & C & 78 \\
\hline MARY'S KUT OR KURL & 222 & 5TH STREET & 623 & C & 40 \\
\hline SPECIAL EFFECT - beauty & 225 & 6TH STREET & 623 & C & 29 \\
\hline ROSE - beauty salon & 340 & SOUTH STREET & 623 & C & 29 \\
\hline SKIN AND HAIR SYSTEMS & 375 & 3RD STREET & 623 & C & 29 \\
\hline BARBER SHOP & 214 & 5TH STREET & 623 & C & 21 \\
\hline ESPINOZA SHOE REPAIR & 439 & SAN BENITO ST & 625 & C & 78 \\
\hline NUMBEROFC & & 41 & & & \\
\hline TOTAL M2 OF C & & 5849 & & & \\
\hline AVE M2 OF C & & 143 & & & \\
\hline$\%$ OF C (AREA) & & 14.2 & & & \\
\hline SDOFC & & 121 & & & \\
\hline
\end{tabular}




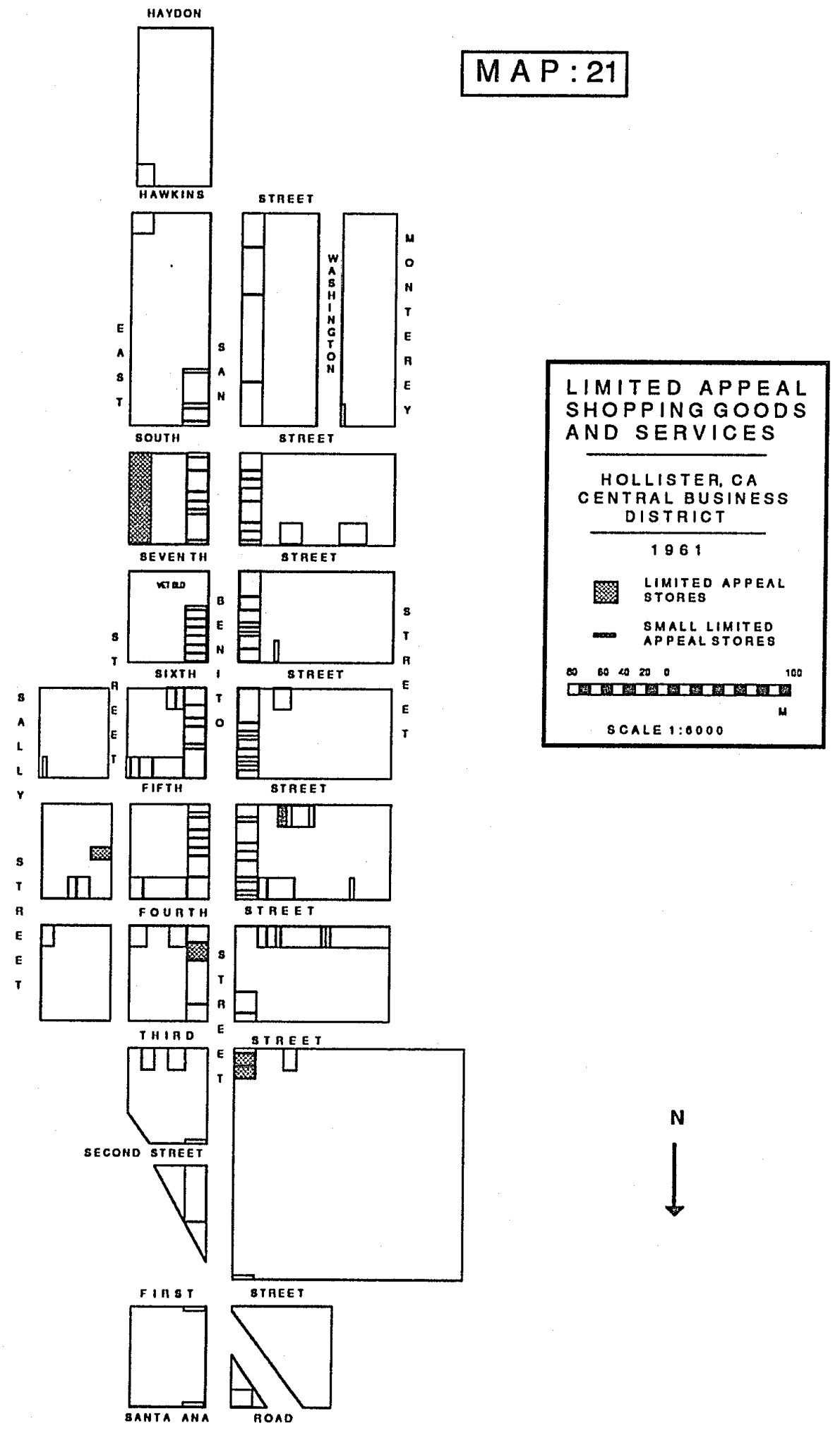


TABLE 29 : LIMITED APPEAL STORES IN HOLLISTER IN 1961 BY STANDARD LAND USE CODE

\begin{tabular}{|c|c|c|c|c|c|c|}
\hline Name of Establishement & & \multicolumn{2}{|c|}{ Street Address } & $\begin{array}{l}\text { Standard } \\
\text { Land Use } \\
\text { Code }\end{array}$ & Type & A:sa $M^{2}$ \\
\hline WESTERN UNION - telegraph & & 334 & 5TH STREET & 472 & $L$ & 60 \\
\hline MCKINNON LUMBER CO & & 217 & TTHSTREET & 521 & $L$ & 506 \\
\hline SAN BENITO GLASS SHOP & 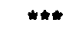 & 238 & SAN BENITO ST & 523 & $L$ & 250 \\
\hline RELLIABLE VENDING MACHINES & & 218 & 5TH STREET & 534 & $L$ & 42 \\
\hline LEE'S HEALTH FOOD CENTER & & 326 & 5TH STREET & 541 & $\mathrm{~L}$ & 96 \\
\hline FEHLMAN'S SADDLE SHOP & $m+$ & 244 & SAN BENITO ST & 596 & $\mathbf{L}$ & 250 \\
\hline BOROVICHPHOTOGRAPHY & & 349 & SAN BENITO ST & 622 & $\mathrm{~L}$ & 236 \\
\hline NUMBER OF L & & & 7 & & & \\
\hline TOTAL M2 OF L. & & & 1500 & & & \\
\hline AVE M2 OF L & & & 214 & & & \\
\hline$\%$ OF L (AREA) & & & 3.9 & & & \\
\hline SDFORL & & & 180 & & & \\
\hline
\end{tabular}

*** Establishment assigned approximate value for area 
TABLE 30 : LIMITED APPEAL STORES IN HOLLISTER IN 1988 BY STANDARD LAND USE CODE

\begin{tabular}{|c|c|c|c|c|}
\hline ne of Establishement & Stres & Address & Standard & $\begin{array}{l}\text { Type Area } M^{2} \\
\text { Land Use } \\
\text { Code }\end{array}$ \\
\hline 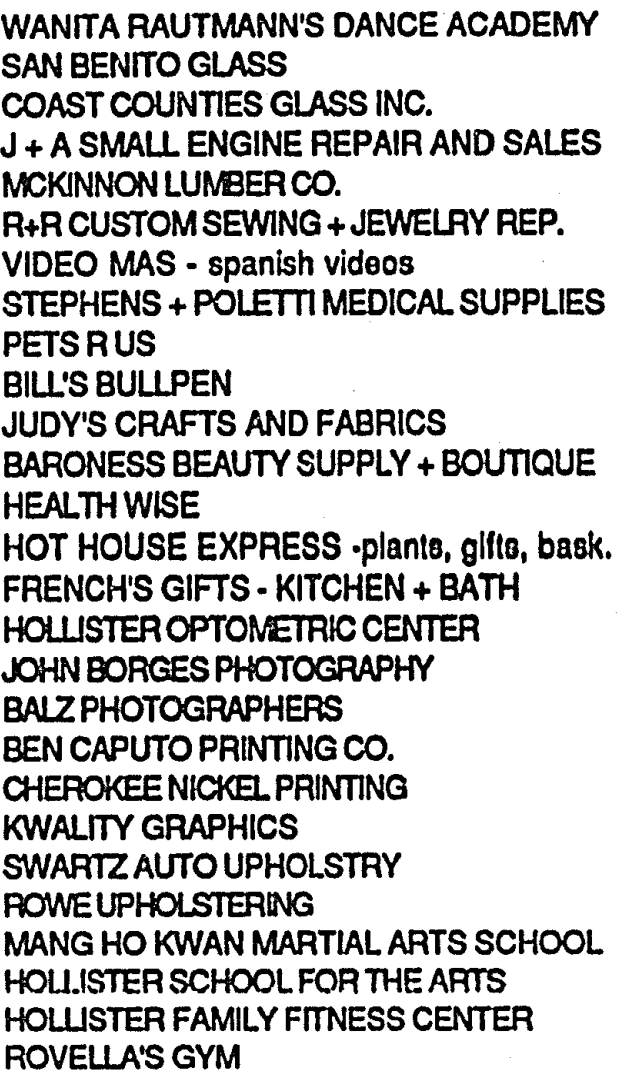 & $\begin{array}{l}211 \\
217 \\
101 \\
139 \\
217 \\
512 \\
320 \\
335 \\
728 \\
414 \\
400 \\
525 \\
627 \\
324 \\
202 \\
347 \\
230 \\
404 \\
330 \\
520 \\
611 \\
125 \\
220 \\
717 \\
721 \\
650 \\
328\end{array}$ & $\begin{array}{l}\text { 6TH STREET } \\
\text { 4TH STREET } \\
\text { 5TH STREET } \\
\text { 4TH STREET } \\
\text { TTH STREET } \\
\text { SAN BENITO ST } \\
\text { 5TH STREET } \\
\text { SAN BENITO ST } \\
\text { SAN BENTO ST } \\
\text { SAN BENTTO ST } \\
\text { SAN BENITO ST } \\
\text { SAN BENTTO ST } \\
\text { SAN BENTTO ST } \\
\text { 5TH STREET } \\
\text { STH STREET } \\
\text { 6TH STREET } \\
\text { 5TH STREET } \\
\text { SAN BENITO ST } \\
\text { SAN BENITO ST } \\
\text { SAN BENTTO ST } \\
\text { SAN BENITO ST } \\
\text { 4TH STREET } \\
\text { 5TH STREET } \\
\text { SAN BENITO ST } \\
\text { SAN GENITO ST } \\
\text { EAST STREET } \\
\text { 4TH STREET }\end{array}$ & $\begin{array}{l}383 \\
523 \\
523 \\
525 \\
527 \\
567 \\
592 \\
599 \\
599 \\
599 \\
599 \\
599 \\
599 \\
599 \\
599 \\
599 \\
622 \\
622 \\
633 \\
633 \\
633 \\
641 \\
649 \\
683 \\
683 \\
742 \\
742\end{array}$ & $\begin{array}{l}260 \\
420 \\
318 \\
266 \\
566 \\
146 \\
96 \\
609 \\
150 \\
136 \\
135 \\
125 \\
50 \\
98 \\
120 \\
125 \\
249 \\
135 \\
210 \\
195 \\
102 \\
187 \\
40 \\
182 \\
182 \\
503 \\
101\end{array}$ \\
\hline $\begin{array}{l}\text { NUMBER OFL } \\
\text { TOTAL M2 OF L } \\
\text { AVE M2 OF L } \\
\% \text { OF L (AREA) } \\
\text { SD FORL }\end{array}$ & & $\begin{array}{l}27 \\
5704 \\
211 \\
13.8 \\
150\end{array}$ & & \\
\hline
\end{tabular}




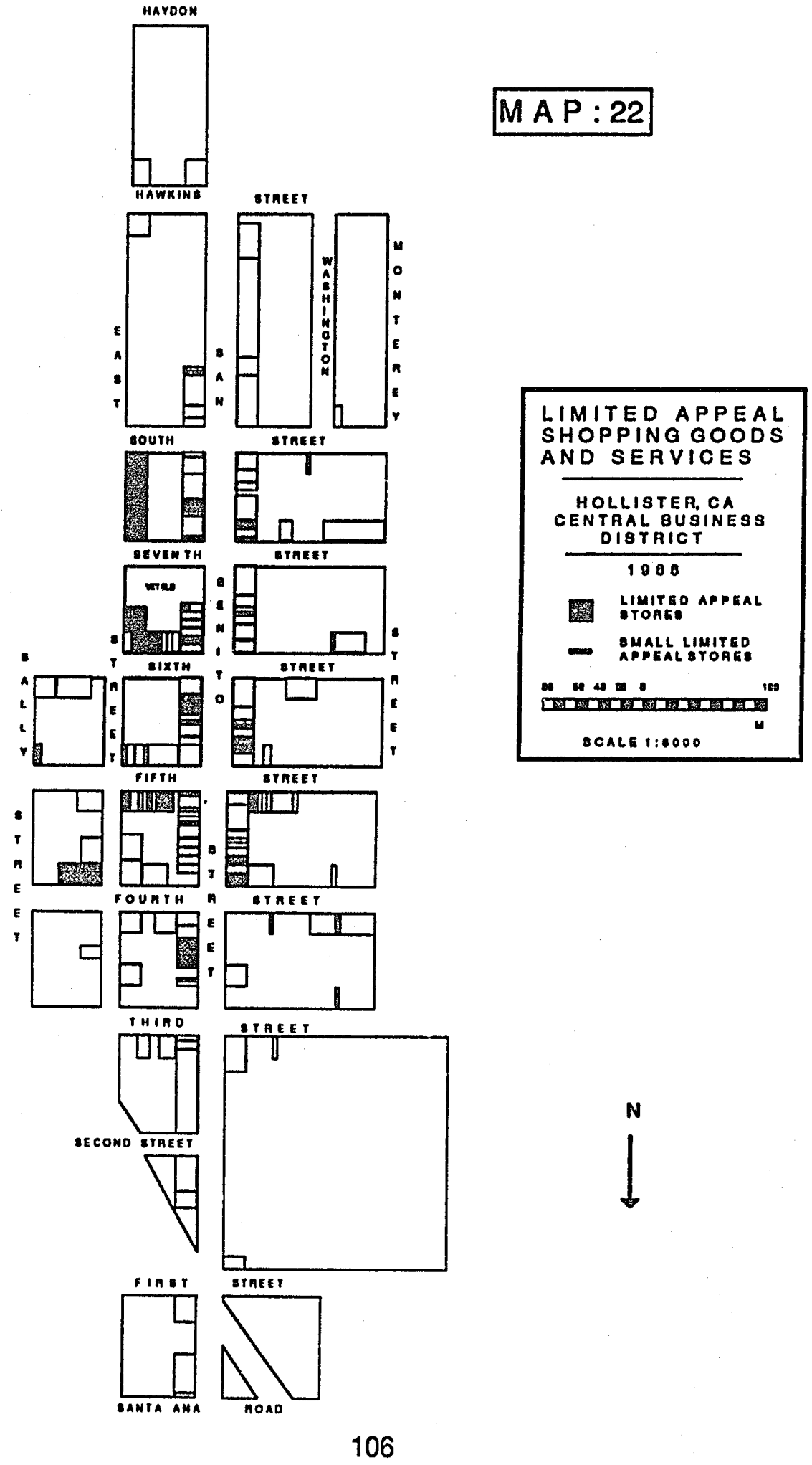


PLEASE NOTE

Page(s) not included with original material and unavailable from author or university.

Filmed as received.

107

University Microfilms International 


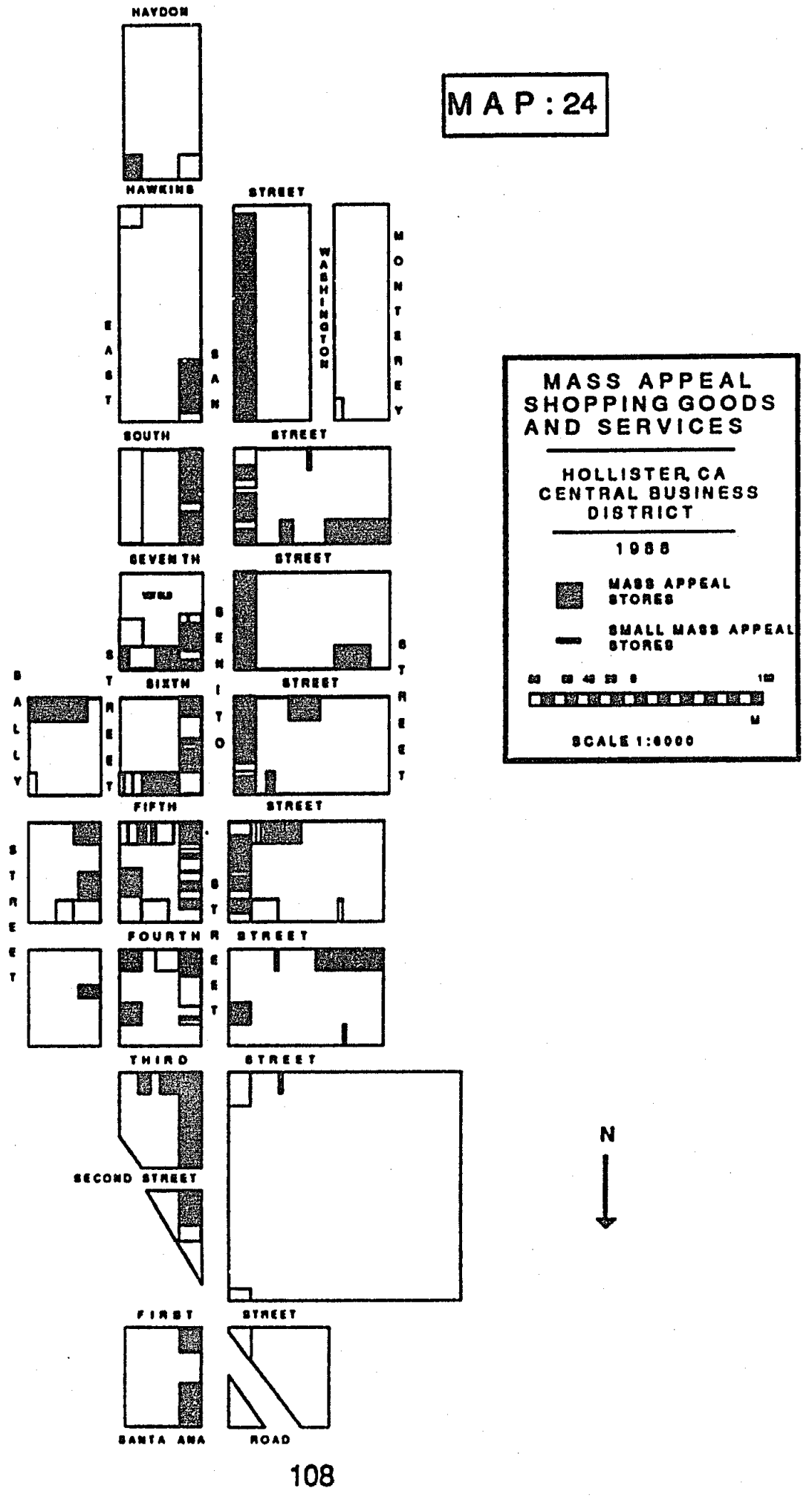


4) The percentage of space used by stores selling mass appeal goods increased only slightly-by less than one percent. In 1988, mass appeal took up $69.4 \%$ of the retail space. Less space off the "main street" was taken up by mass appeal stores in 1988 than in 1961. During these 28 years, changes within the mass appeal category have also been significant. Restaurants in 1961 took up only $4.5 \%$ of the mass appeal space, but now constitute $12.1 \%$ of the space. The service part of mass appeal (the 600s in the Standard Land Use Ceding Manual) has increased greatly. In 1961, there was only one bank in Hollister; now there are four. This is a change from only one percent to almost eight percent of the total mass appeal space. (see tables 31 and 32 )

\section{The Moin Street - San Benito Street}

San Benito Street, from Haydon Street to Santa Ana Road, is considered the "main street" in Hollister. Just over $50 \%$ of the total retail area in the downtown is on the "main street". The "main" street in 1960 had slightly less convenience space and significantly less limited appeal space than the whole downtown has. (see tables 33 and 34) The limited appeal space to convenience space ratio for the main street is only .06. There is 17.5 times more convenience space than limited appeal space.

From 1961 to 1988, the percentage of limited appeal space on the main street increased from just over one percent to ten percent. The percentage of convenience space decreased even more than it did for the whole downtown. In 1988, the percentage of convenience space 
TABLE 31 : MASS APPEAL STORES IN HOLLISTER IN 1961 BY STANDARD LAND USE CODE

Name of Establishement

GREYHOUND - bus station

ACME TAXI

LADD HARDWARE STORE

BROWN \& CHAPPELL CO - hardware

BAUGHMAN'S DEPT STORE

SCHULZE DEPT STORE

J C PENNY CO - dept

RASCO DEPT STORE

CORONET VARIETY STORE

HOLTS VARIETY STORE

TRI-WAY MARKET

SHOPPERS SUPER MARKET *

HOLLISTER BAKERY

MODEL BAKERY

BISHOP'S AUTO DEALERS

HOLLISTER GARAGE AUTO DLR

JONES CHEVROLET - new cars "**

JONES CHEVROLET - used cars ***

TIFFANY AUTO DEALERS

ANDERSON TIRE SERVICE

HOLLISTER AUTO PARTS - retail

ABBS AUTO SUPPLY

WESTERN AUTO SUPPLY

SCHIPPER'S - men's clothes

WARDROBE - men's clothes

MEN'S MART - clothes

LEPAINE'S MEN'S WEAR

MCCONNELL \& O'NIEL - men's clothes

MODE O DAY - women's clothes

TOWNTRY FASHIONS-women's "**

AD-DOE FASHIONS - women's clothes

BOOTERY THE - shoos

HAROLD'S SHOES

SHAWS SHOE STORE

BLACK-COOPERFURN CO

HOLLISTER FURNITURE CO

GUNNELL FUAN STORE

P\&L USED FURNITURE

ED'S FURNITURE - used furn

ROWE'S UPHOLSHOP

BOBS TV \& APPLS

ROYAL REFGR CO - appliances

COLBURN'S RADIO \& TV
Street Address

Standard Type Area $M^{2}$

Land Use

Code

\section{0}

417

620

328

544

340

310

528

221

642

829

212

750

537

201

396

372

371

800

335

139

400

609

610

515

414

404

619

422

440

625

411

512

220

648

731

813

730

250

206

894

230

217

110

$\begin{array}{llll}\text { 4TH STREET } & 421 & \mathrm{M} & 79 \\ \text { SAN BENITO ST } & 429 & \mathrm{M} & 141 \\ \text { SAN BENITO ST } & 525 & \mathrm{M} & 320 \\ \text { 4TH STREET } & 525 & \mathrm{M} & 101 \\ \text { SAN BENITO ST } & 531 & \mathrm{M} & 1163 \\ \text { 6TH STREET } & 531 & \mathrm{M} & 408 \\ \text { 4TH STREET } & 531 & \mathrm{M} & 250 \\ \text { SAN BENITO ST } & 531 & \mathrm{M} & 250 \\ \text { 5TH STREET } & 533 & \mathrm{M} & 696 \\ \text { SAN BENITO ST } & 533 & \mathrm{M} & 242 \\ \text { SAN BENITO ST } & 541 & \mathrm{M} & 1106 \\ \text { 6TH STREET } & 541 & \mathrm{M} & 250 \\ \text { SAN EENITO ST } & 546 & \mathrm{M} & 174 \\ \text { SAN BENITO ST } & 546 & \mathrm{M} & 165 \\ \text { SAN BENITO ST } & 551 & \mathrm{M} & 2970 \\ \text { 4TH STREET } & 551 & \mathrm{M} & 1370 \\ \text { 4TH STREET } & 551 & \mathrm{M} & 939 \\ \text { 4TH STREET } & 551 & \mathrm{M} & 600 \\ \text { SAN BENITO ST } & 551 & \mathrm{M} & 245 \\ \text { SAN BENITO ST } & 552 & \mathrm{M} & 609 \\ \text { 4TH STREET } & 552 & \mathrm{M} & 266 \\ \text { SAN BENITO ST } & 552 & \mathrm{M} & 135 \\ \text { SAN BENITO ST } & 552 & \mathrm{M} & 116 \\ \text { SAN BENITO ST } & 561 & \mathrm{M} & 254 \\ \text { SAN BENITO ST } & 561 & \mathrm{M} & 250 \\ \text { SAN BENITO ST } & 561 & \mathrm{M} & 136 \\ \text { SAN BENITO ST } & 561 & \mathrm{M} & 135 \\ \text { SAN BENITO ST } & 561 & \mathrm{M} & 102 \\ \text { SAN BENITO ST } & 562 & \mathrm{M} & 421 \\ \text { SAN BENITO ST } & 562 & \mathrm{M} & 159 \\ \text { SAN BENITO ST } & 562 & \mathrm{M} & 93 \\ \text { SAN BENITO ST } & 566 & \mathrm{M} & 250 \\ \text { SAN BENITO ST } & 566 & \mathrm{M} & 146 \\ \text { 5TH STREET } & 566 & \mathrm{M} & 40 \\ \text { SAN BENITO ST } & 571 & \mathrm{M} & 611 \\ \text { SAN BENITO ST } & 571 & \mathrm{M} & 549 \\ \text { SAN BENITO ST } & 571 & \mathrm{M} & 422 \\ \text { SAN BENITO ST } & 571 & \mathrm{M} & 408 \\ \text { SAN BENITO ST } & 571 & \mathrm{M} & 60 \\ \text { 5TH STREET } & 571 & \mathrm{M} & 139 \\ \text { SAN BENITO ST } & 572 & \mathrm{M} & 250 \\ \text { 5TH STREET } & 572 & \mathrm{M} & 249 \\ \text { 4TH STREET } & 573 & \mathrm{M} & 840\end{array}$




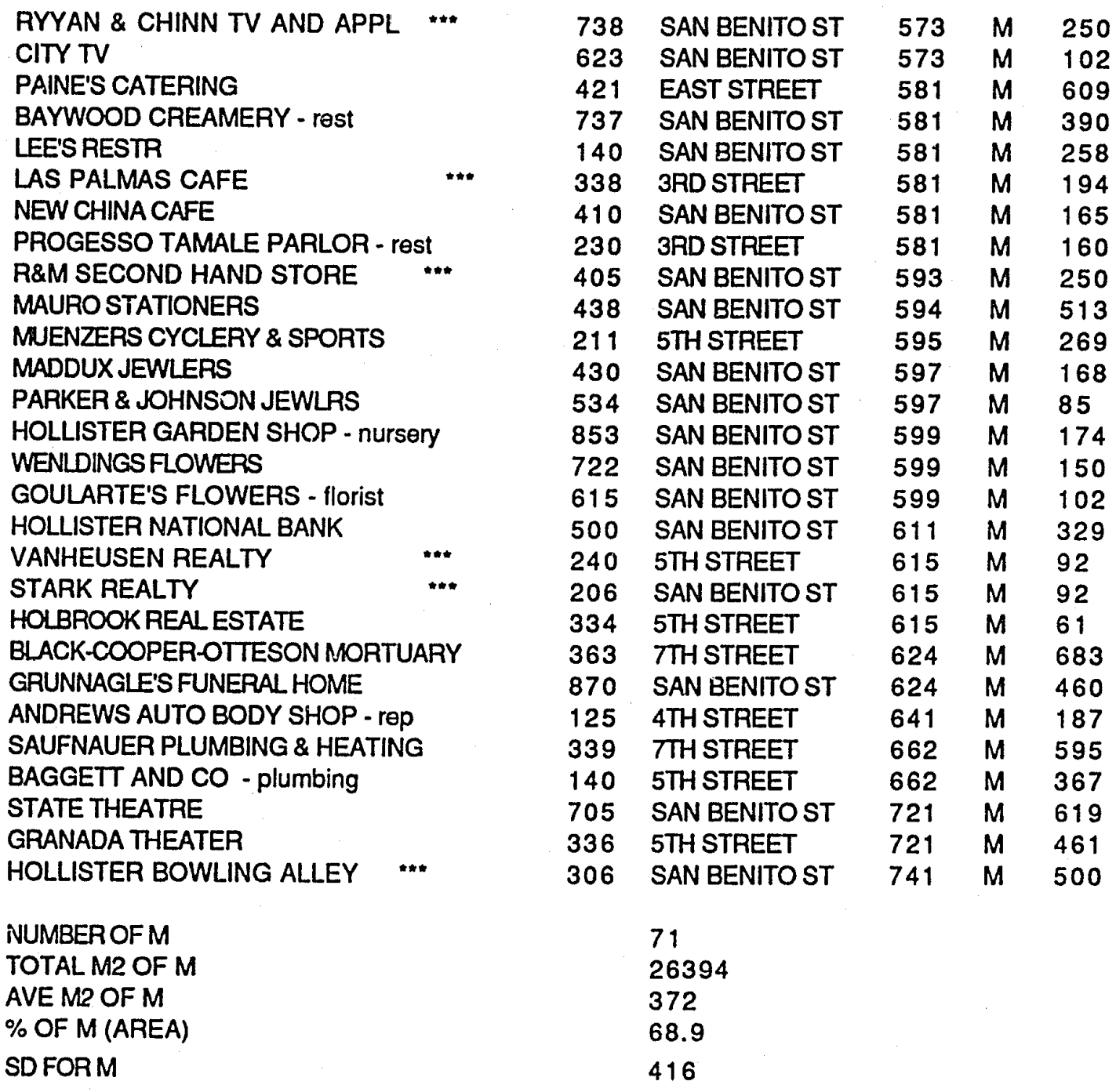

.** Establishment assigned approximate value for area 
TABLE 32 : MASS APPEAL STORES IN HOLLISTER IN 1988 BY STANDARD LAND USE CODE

\begin{tabular}{|c|c|c|c|c|c|}
\hline Name of Establishement & Stres & Address & $\begin{array}{l}\text { Standar } \\
\text { Land Us } \\
\text { Codo }\end{array}$ & & Area \\
\hline FAST TRAVEL & 327 & 5TH STREET & 492 & $M$ & 182 \\
\hline HOLLISTER TRAVEL & 530 & SAN BENITO ST & 492 & $M$ & 85 \\
\hline BAUDER CO. PLUMBING + HEATING & 140 & 5TH STREET & 522 & $\mathbf{M}$ & 367 \\
\hline RADIO SHACK & 623 & SAN BENITO ST & 524 & $M$ & 102 \\
\hline TRUE VALUE HARDWARE & 339 & TTHSTREET & 525 & M & 595 \\
\hline ACE HARDWARE & 620 & SAN BENITO ST & 525 & $M$ & 320 \\
\hline J. C. PENNY - dept store & 372 & 4TH STREET & 531 & $M$ & 939 \\
\hline FORD'S - dept store & 544 & SAN BENITO ST & 531 & $M$ & 698 \\
\hline SEARS-CATOLOG SALES & 713 & SAN BENITO ST & 531 & $M$ & 111 \\
\hline BALERGROSERY & 211 & 3RD STREET & 541 & M & 1103 \\
\hline CHOCOLATE PEDDLERS & 243 & 6TH STREET & 544 & $M$ & 78 \\
\hline EL NOPAL BAKERY + TORTILLA FACTORY & 216 & 3RD STREET & 546 & $M$ & 236 \\
\hline BISHOP MOTORS - sales and repair & 201 & SAN BENITO ST & 551 & $M$ & 2970 \\
\hline TIFFANY MOTOR CO. & 800 & SAN BENITO ST & 551 & $M$ & 245 \\
\hline TOP QUALITY USED CARS & 125 & SAN BENITO ST & 551 & $M$ & 34 \\
\hline STAR AUTO SUPPLY & 55 & SAN BENITO ST & 552 & $M$ & 495 \\
\hline KELLY'S AUTO PARTS & 203 & HAWKINS ST & 552 & $M$ & 314 \\
\hline HOLLISTER AUTO PARTS & 140 & 4TH STREET & 552 & $M$ & 302 \\
\hline LACEY AUTO PARTS & 120 & 6TH STREET & 552 & $\mathbf{M}$ & 187 \\
\hline DICK BRUHN MEN'S CLOTHES & 517 & SAN BENITO ST & 561 & $M$ & 250 \\
\hline FASHION ENCORE- women's clothes & 420 & SAN BENITO ST & 562 & $M$ & 148 \\
\hline FASION TREE - women's clothes & 535 & SAN BENITO ST & 562 & M & 124 \\
\hline SHIRLEY'S - women's cloths & 619 & SAN BENITO ST & 562 & $M$ & 102 \\
\hline PEPPERMINT PONY-childrens clothes & 628 & SAN BENITO ST & 564 & $M$ & 156 \\
\hline GINGERBREAD HOUSE - children's cloths & 334 & 5TH STREET & 564 & $M$ & 61 \\
\hline WE R KIDS - children's clothes & 334 & 5TH STREET & 564 & $M$ & 60 \\
\hline FAMILY BARGAIN CENTER - clothes & 725 & SAN BENITO ST & 565 & M & 579 \\
\hline FOF:D'S - clothes & 340 & GTH STREET & 565 & $M$ & 408 \\
\hline SCHIPPERS' CLOTHES & 610 & SAN BENITO ST & 565 & $M$ & 254 \\
\hline PANTS PLACE & 355 & SAN BENITO ST & 565 & $\mathbf{M}$ & 236 \\
\hline HOUSTER WESTER & 722 & SAN BENITO ST & 565 & $\mathbf{M}$ & 150 \\
\hline MODE O' DAY - clothes & 459 & SAN BENITIO ST & 565 & $M$ & 106 \\
\hline DICK BRUHN SHOES & 515 & SAN BENITO ST & 566 & $M$ & 250 \\
\hline SHOE PLACE & 349 & SAN BENITO ST & 566 & $M$ & 236 \\
\hline BLACK - COOPER FURNITURE & 648 & SAN BENITO ST & 571 & $\mathbf{M}$ & 611 \\
\hline CHARLIE'S INTERIORS-drapes, wallpaper & 730 & SAN BENITO ST & 571 & $M$ & 408 \\
\hline RON'S WINDOWS, WALLS + FLOORS INC. & 219 & SAN BENITO ST & 571 & $M$ & 395 \\
\hline FACTORY DIRECT FURNITURE & 750 & SAN BENITO ST & 571 & $M$ & 174 \\
\hline FORTINO'S APPLIANCES & 829 & SAN BENITO ST & 572 & $\mathbf{M}$ & 1106 \\
\hline ENTERPRISE ELECTRIC CC & 542 & SAN BENITO ST & 572 & $M$ & 270 \\
\hline PAINE'S RESTUARANT AND COCKTAILS & 421 & EAST STREET & 581 & $M$ & 609 \\
\hline SAN ANDREAS BREWING CO. & 737 & SAN BENITO ST & 581 & $M$ & 390 \\
\hline KENTUKY FRIED CHICKEN & 140 & SAN BENITO ST & 581 & $M$ & 258 \\
\hline LAS PALMAS - rest. & 445 & SAN BENITO ST & 581 & $M$ & 217 \\
\hline DON PEDRO'S MEXICAN RESTUARANT & 204 & 4TH STREET & 581 & $M$ & 214 \\
\hline
\end{tabular}




\begin{tabular}{|c|c|c|c|c|c|}
\hline$A+W=$ rest & 203 & SOUTH STREET & 581 & $M$ & 192 \\
\hline NEW CHINA CAFE & 410 & SAN BENITO ST & 581 & $M$ & 165 \\
\hline PROGRESSOREST. & 230 & 3RD STREET & 581 & $M$ & 160 \\
\hline COZY CUP CAFE & 380 & 4TH STREET & 581 & $\mathbf{M}$ & 159 \\
\hline JOHNNIE'S BAR AND GRILLL & 526 & SAN BENITO ST & 581 & $M$ & 148 \\
\hline PARADISE INN - rest & 905 & HAWKINS ST & 581 & $M$ & 148 \\
\hline KIM'S PLACE - rest. & 419 & SAN BENITO ST & 581 & $M$ & 141 \\
\hline NINO'S PIZZA & 419 & SAN BENITO ST & 581 & $M$ & 141 \\
\hline WOODEN SPOON - reSt & 257 & 6TH STREET & 581 & $M$ & 116 \\
\hline DELI, THE & 636 & SAN BENITO ST & 581 & $M$ & 115 \\
\hline CHEUNG SHENG RESTUARANT & 201 & 6TH STREET & 581 & $\mathbf{M}$ & 105 \\
\hline DOMINO'S PIZZA & 615 & SAN BENITO ST & 581 & $M$ & 102 \\
\hline HAPPY DOGS - rest. & 441 & SAN BENITO ST & 581 & $M$ & 78 \\
\hline NEW AND USED & 813 & SAN BENITO ST & 593 & $\mathbf{M}$ & 422 \\
\hline MAURO STATIONERS-office equip. & 438 & SAN BENITO ST & 594 & $M$ & 513 \\
\hline BOOKSTORE, THE & 642 & SAN BENITO ST & 594 & $M$ & 242 \\
\hline MUENZER CYCLERY + SPORTS CENTER & 221 & 5TH STREET & 595 & $M$ & 696 \\
\hline MADDOX JEWELRY & 430 & SAN BENITO ST & 597 & $M$ & 168 \\
\hline JOHNSON JEWELERS & 534 & SAN BENITO ST & 597 & $M$ & 85 \\
\hline HOUISTER-GOULARTE FLORAL & 609 & SAN BENITO ST & 599 & $M$ & 116 \\
\hline COUNTRY VILLAGE FLORIST & 850 & SAN BENITO ST & 599 & $\mathbf{M}$ & 105 \\
\hline BANK OF AMERICA & 320 & SAN BENITO ST & 611 & $\mathbf{M}$ & 850 \\
\hline WELIS FARGO BANK & 375 & 6TH STREET & 611 & M & 546 \\
\hline WORLD SAVING & 575 & SAN BENITO ST & 611 & M & 413 \\
\hline CALIFORNIA FIRST BANK & 500 & SAN BENITO ST & 611 & $M$ & 329 \\
\hline COMTEMPO REALTY & 898 & SAN BENITO ST & 615 & $M$ & 182 \\
\hline J.B. HOWARD REAL ESTATE & 420 & SAN BENITO ST & 615 & $M$ & 125 \\
\hline WAGNER REAL ESTATE & 350 & 5TH STREET & 615 & $M$ & 108 \\
\hline BOZZO REALESTATE & 321 & SAN BENITO ST & 615 & $M$ & 78 \\
\hline DE ROZA REAL ESTATE & 843 & SAN BENITO ST & 615 & M & 77 \\
\hline HALL REAL ESTATE & 540 & MONTEREYST & 615 & $M$ & 77 \\
\hline PIONEERREALITY & 218 & 5TH STREET & 615 & M & 42 \\
\hline BLACK, COOPER, SANDER FUNERAL HOME & 363 & 7TH STREET & 624 & $M$ & 683 \\
\hline GRUNNAGLE-AMENT-NELSON FUNERAL H. & 870 & SAN BENITO ST & 624 & $M$ & 460 \\
\hline HOLLISTER GARAGE - auto repair & 396 & 4TH STREET & 641 & $M$ & 1370 \\
\hline TEXACO - auto repair no gas & 759 & SAN BENITO ST & 641 & M & 26 \\
\hline$T+T$ AUTO REPAIR & 130 & 6TH STREET & 641 & M & 293 \\
\hline EAST STREET SERVICE CENTER - auto rep & 424 & EAST STREET & 641 & $M$ & 279 \\
\hline HOLLISTER TRANSMISSION + AUTO REP. & 5 & SAN BENITO ST & 641 & $M$ & 154 \\
\hline ANDRE RADIATOR SERVICE & 336 & 3RD STREET & 641 & $M$ & 94 \\
\hline HOLLISTER MUFFLERS & 249 & SAN BENITO ST & 641 & M & 93 \\
\hline SHOW CASE MOVIE THEATER & 705 & SAN BENITO ST & 721 & $\mathrm{M}$ & 619 \\
\hline GRANADA THEATER & 336 & 5TH STREET & 721 & M & 461 \\
\hline NUMBEROFM & & 88 & & & \\
\hline TOTAL M2 OF M & & 28611 & & & \\
\hline AVE M2 OF M & & 325 & & & \\
\hline$\%$ OF $M$ (AREA) & & 69.4 & & & \\
\hline SDFORM & & 387 & & & \\
\hline
\end{tabular}


TABLE 33: STATISTICS FOR HOLLISTEA'S CBD

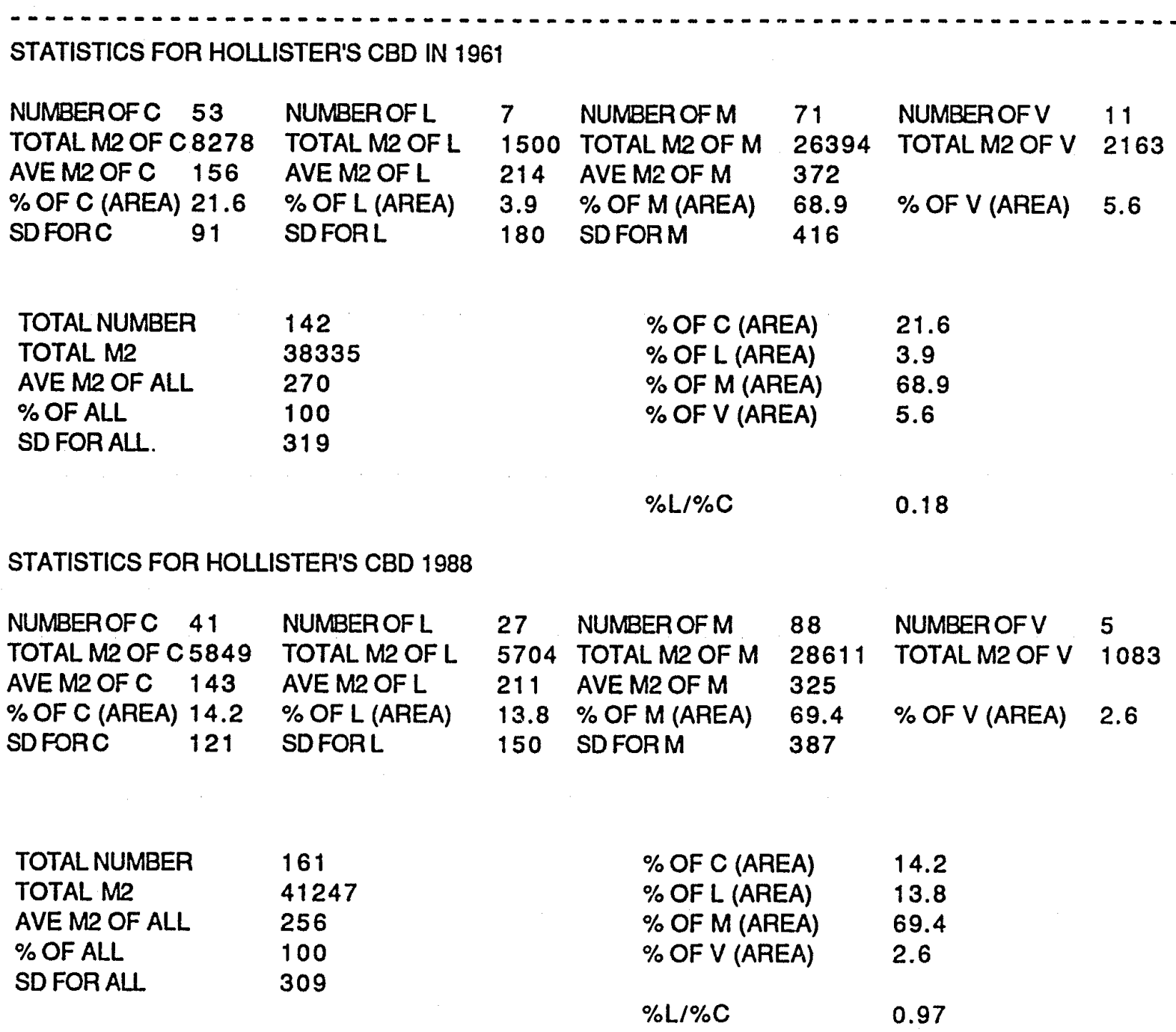

CHANGE FROM 1961 TO 1988

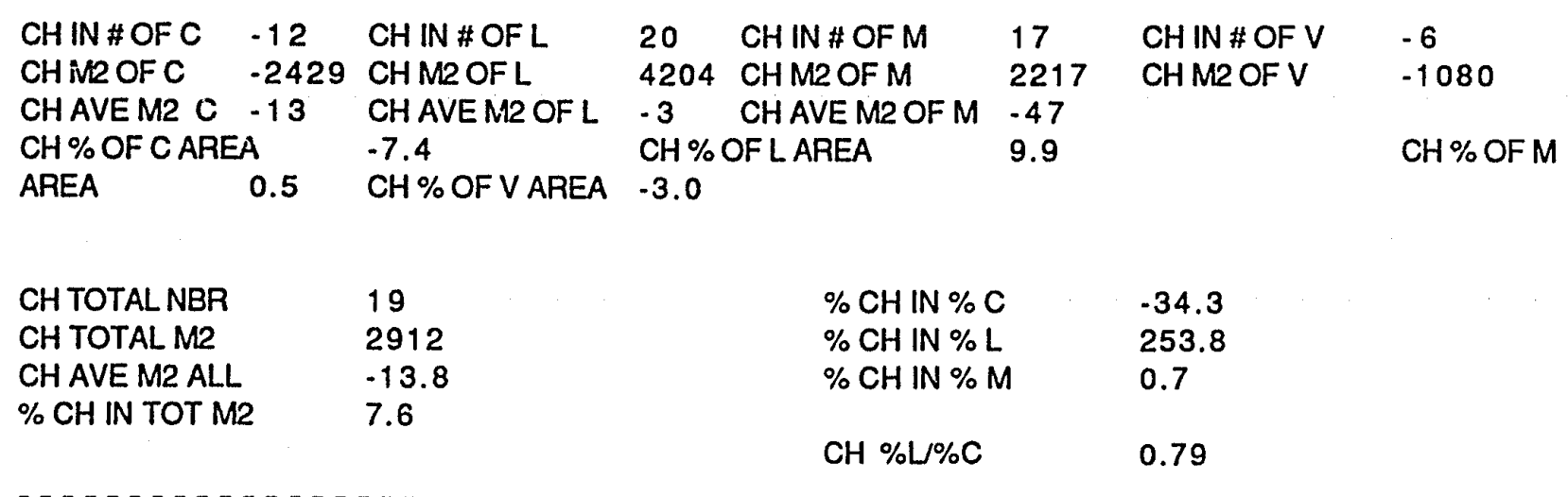


TABLE 34: STATISTICS FOR HOLLISTER'S "MAIN STREET"

STATISTICS FOR HOLLISTER'S "MAIN STREET" IN 1961

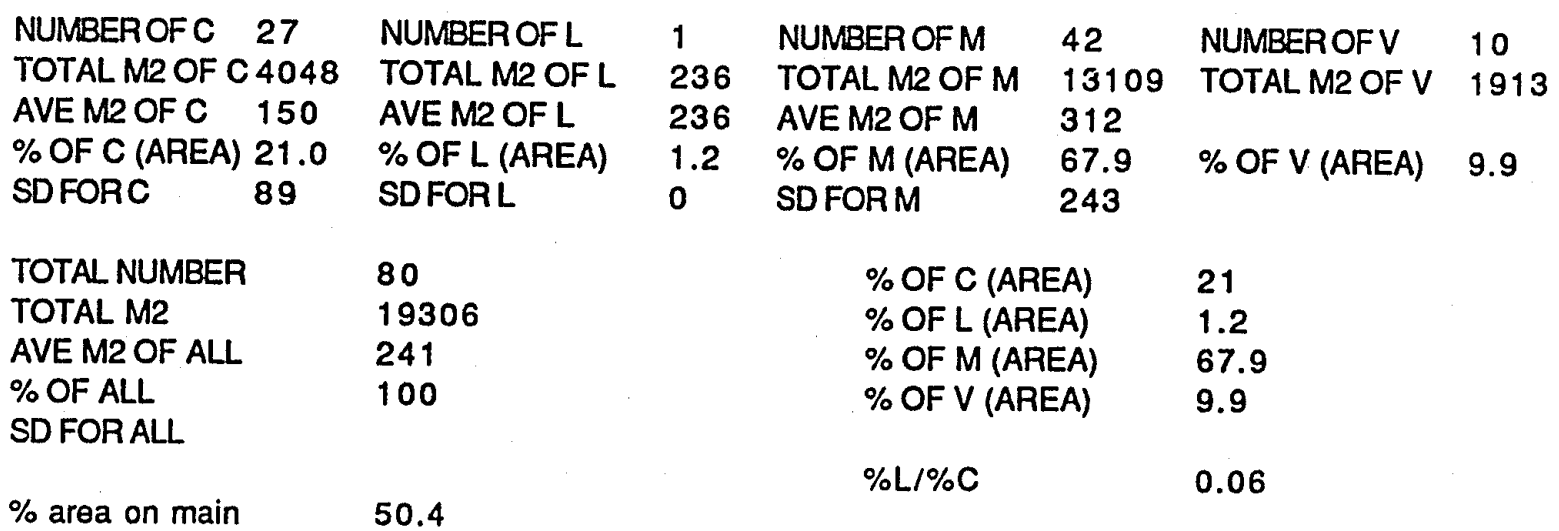

STATISTICS FOR HOLLISTER'S MAIN STREET IN 1988

$\begin{array}{llllllll}\text { NUMBEROFC } & 21 & \text { NUMBER OFL } & 13 & \text { NUMBER OFM } & 56 & \text { NUMBEROFV } & 3 \\ \text { TOTAL M2 OF C } 3075 & \text { TOTAL M2 OF L } & 2357 & \text { TOTAL M2 OFM } & 17427 & \text { TOTAL M2 OF V } & 605 \\ \text { AVE M2 OF C } & 146 & \text { AVE M2 OF L } & 181 & \text { AVE M2 OF M } & 311 & & \\ \text { \% OF C (AREA) } & 13.1 & \% \text { OF L (AREA) } & 10.0 & \% \text { OF M (AREA) } & 74.3 & \% \text { OF V (AREA) } & 2.6 \\ \text { SD FORC } & 132 & \text { SDFORL } & 135 & \text { SDFORM } & 420 & & \end{array}$

TOTAL NUMBER $93 \quad \%$ OF C (AREA) 13.1

TOTAL M2 $23464 \%$ OF L (AREA) 10

AVE M2 OF ALL $252 \%$ OF M (AREA) 74.3

$\%$ OF ALL $\quad 100$

$\%$ OF V (AREA)

2.6

SD FORALL $\quad 343$

$\%$ area on main 56.8

$\% L / \% C$

0.76

CHANGE IN THE MAIN STREET FROM 1961 TO 1988

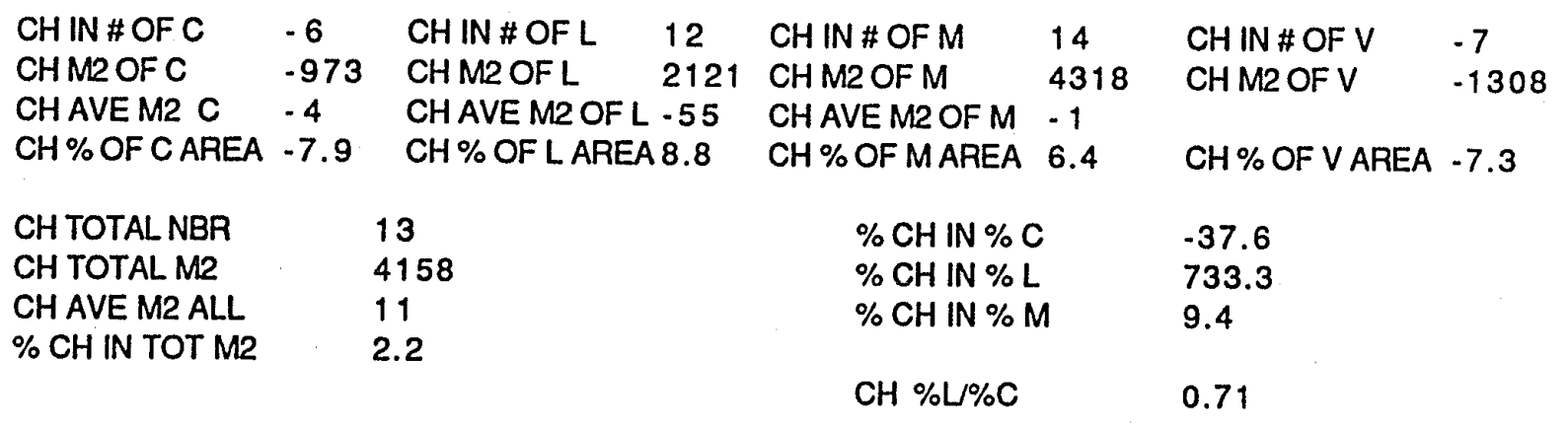


decreased to $13.1 \%$. Vacant space decreased from $9.9 \%$ to only $2.6 \%$. Most of this space was filled by mass appeal stores, which increased their percentage of the total floorspace from $67.9 \%$ to $74.3 \%$. The sum of limited appeal space and convenience space increased by less than one percent. Although the space occupied by establishments selling limited appeal goods increased on both the "main street" and in downtown as a whole, the percent of limited appeal space is significantly less on the "main street" than for the downtown as a whole. Although San Benito Street has $56.8 \%$ of the total retail floorspace, it contains only $41.3 \%$ of the total limited appeal space. 


\section{CHAPTER IV}

\section{A COMPARISON OF ALL THE CITIES}

The same trend in retail development is observable in Los Gatos, Santa Cruz, Watsonville, and Hollister, but the cities are at different stages. From 1960 to 1988 , they have all increased the percentage of limited appeal space and decreased the percentage of convenience space in their Central Business District retail areas. Although the percentage of mass appeal space is affected by this trend in retail developement, it varies more with the amount of retail that exists outside of the Central Business District.

The sum of the percentage of limited appeal space and the percentage of convenience space $(L+C)$ is an inverse function of the amount of retail space that exists outside of the CBD. If there is a lot of retail space outside of the CBD, then there will be relatively less limited appeal and convenience space within the CBD. If there is very little retail space outside of the $\mathrm{CBD}$, then there will be comparatively more limited and convenience space within the CBD. Since Hollister has very little retail space outside of the CBD area, it has more limited appeal space and convenience space in the CBD.

Santa Cruz has a large amount of retail outside of the CBD and, therefore, has a smaller proportion of convenience and limited appeal space. The significant growth of retail space outside of the CBD between 1960 and 1988 in Los Gatos, however, has not been 
accompanied by an increase in the percentage of mass appeal space because of the huge growth in limited appeal space. Los Gatos is evolving into a limited appeal specialty shopping area.

The largest growth in percentage of mass appeal space has been in Watsonville (11.2\%), which has experienced considerable growth in the northern part of the city. The next largest percent increase has been in Santa Cruz (4.5\%) which is also growing significantly outside of the CBD. Hollister has grown only slightly outside of the downtown area and shows only a slight increase in percent of mass appeal area: $0.5 \%$. Los Gatos has a $1.2 \%$ decrease in mass appeal percentage, although it has a slight increase in total floorspace. Since Hollister appears to be starting to grow outside of the CBD, it is likely that some proportion of limited appeal and convenience space will move away from the downtown.

In figure 5, the proportionating of the CBDs for the various cities in 1960, appears to be fairly similar. They all have about $2 / 3$ mass appeal space. Convenience space is quite large for all the cities. The most noticeable difference among the cities is the large amount of vacant space in Watsonville. Although some amount of vacant space is needed in a dynamic society, this high an amount appears unhealthy.

By comparing figures 5 and 6 , one notices that the breakup of retail by type on the "main street" is more like the composition of the entire CBD of that city than it is to the other "main streets". The differences between the entire CBD and the "main street" for each 
Fig. 5

Percentages of retail land use in the CBD

$1960-1961$

HOLLISTER 1961

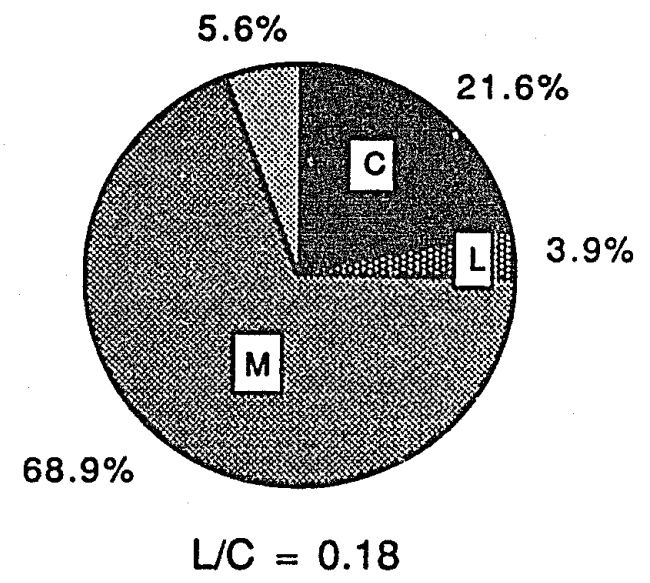

SANTA CRUZ 1960

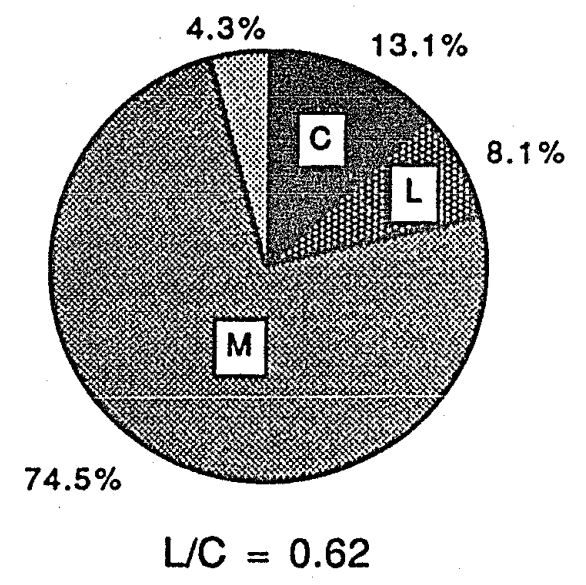

WATSONVILLE 1960

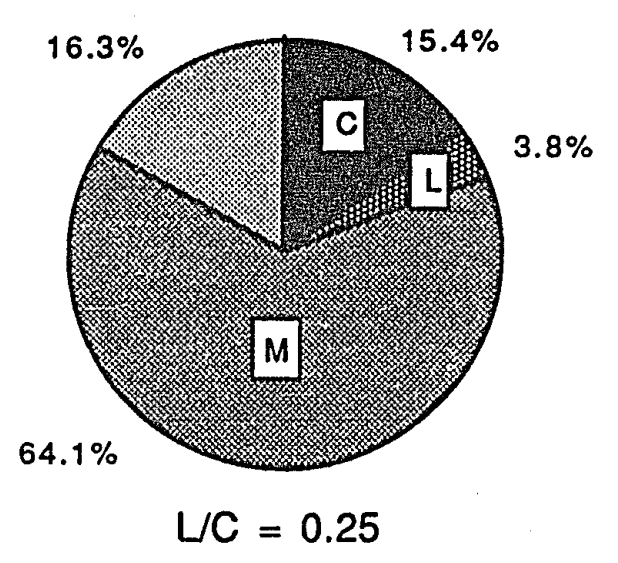

LOSGATOS 1960

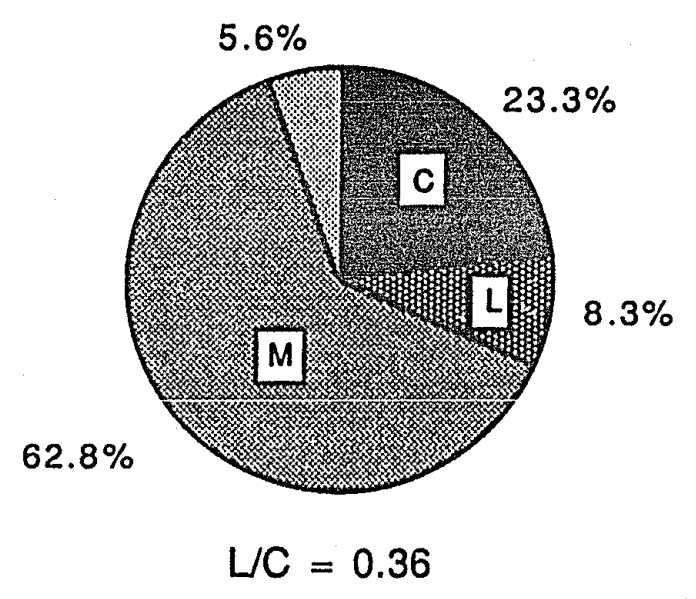


Fig. 6

Percentages of retail land use on

THE MAIN STREETS 1960-1961

HOLLISTER 1961

THE MAIN ST ONLY

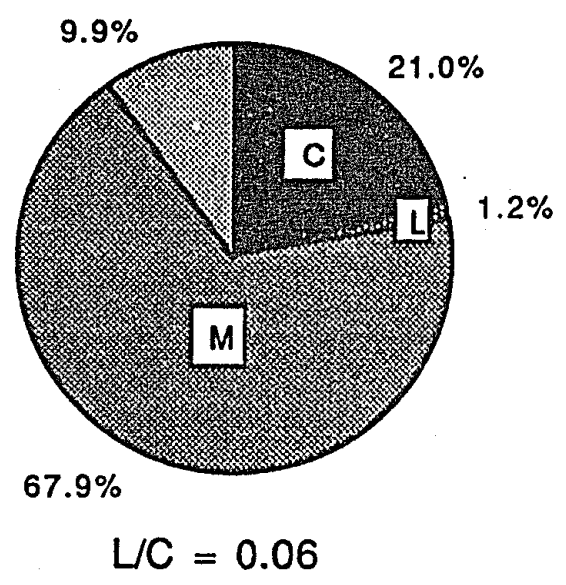

SANTACRUZ 1960

THE MAIN STREET ONLY

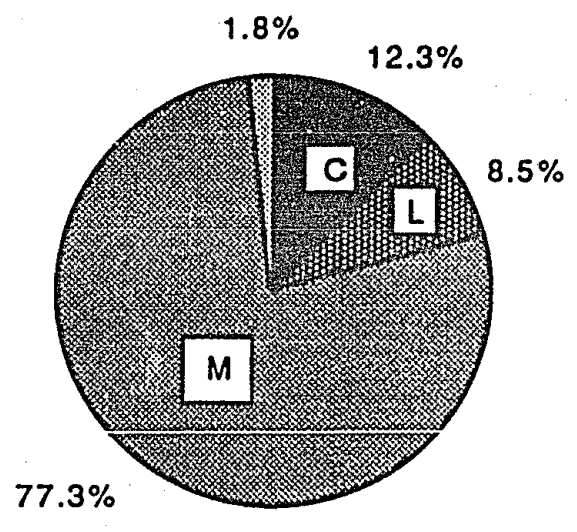

$$
L / C=0.69
$$

WATSONVILLE 1960

THE MAIN ST ONLY

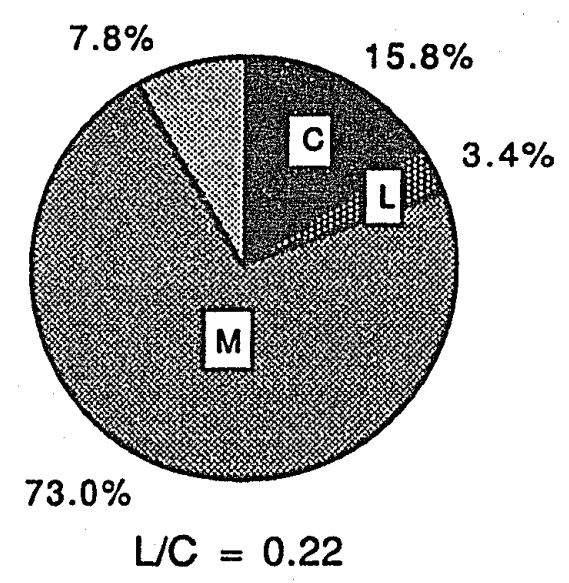

THE MAIN STREET ONLY

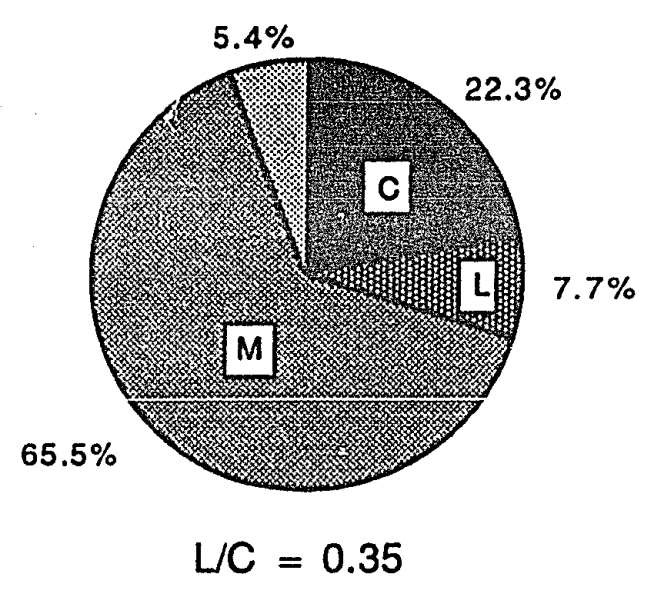


city are quite comparable. The "main streets" have a slightly greater percentage of mass appeal space and a slightly smaller percentage of both limited appeal and convenience space. This is true for 1988, as well as for 1960. (see fig. 7 and 8)

The comparison of the pie graphs for 1988 with the pie graphs for 1960 clearly shows the trend all of the CBDs are following. (see fig 5 and 7) All of the CBDs show a substantial increase in the percentage of limited appeal space. This decrease was so great that in all cases it reflects an absolute drop in convenience floorspace. The percentage of limited appeal space grew considerably in all of the CBDs over this time period. As a result of these changes, the L/C ratio for all the cities increased. The limited appeal/convenience space ratio is particularly important because it shows the extent of the movement away from convenience stores toward limited appeal stores. It also reduces the effect of varying amounts of retail outside of the CBD. Although Santa Cruz and Los Gatos had higher L/C ratios in 1960 than Hollister or Watsonville, the division between the cities is not as distinct in 1960 as it is in 1988. In 1960, the ratio for Hollister was 0.18 , Watsonville was 0.25 , Los Gatos was 0.36 , and Santa Cruz was 0.62 . By 1988 , the two groups are much more distinct. The $L / C$ ratio for Watsonville was 0.91 and for Hollister it was 0.97 . This means that there is almost an equal amount of both limited and convenience space. The CBDs of Los Gatos and Santa Cruz both greatly surpassed the point of even distribution. The ratio for 
Fig. 7

\section{Percentages of retail land use in the CBD 1988}

HOLLISTER 1988

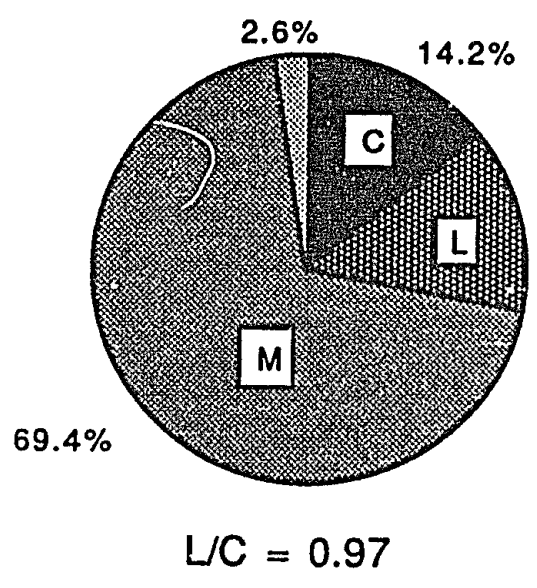

SANTACRUZ 1988

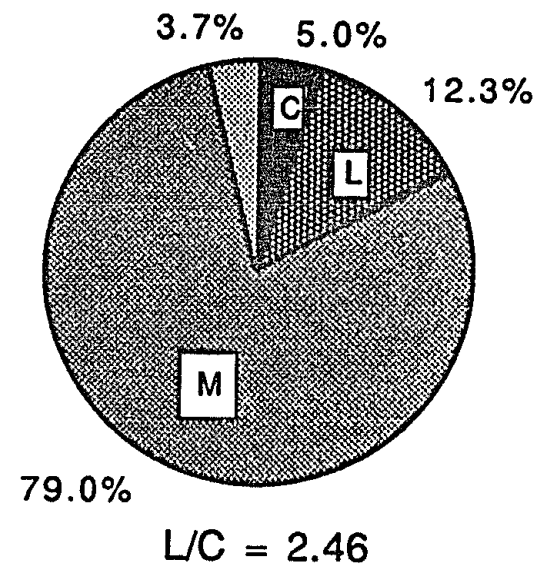

WATSONVILLE 1988

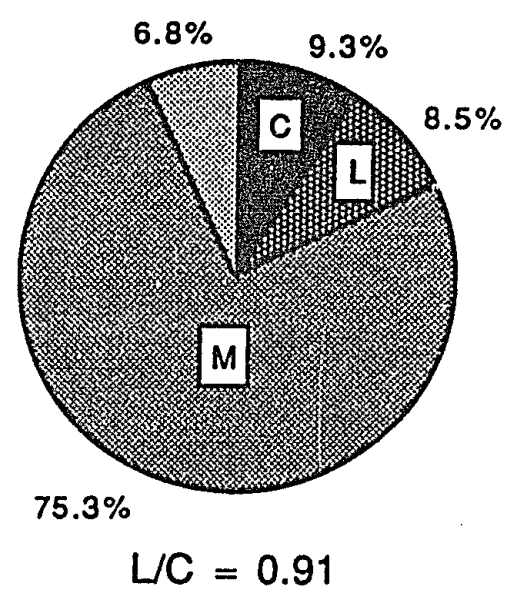

LOSGATOS 1988

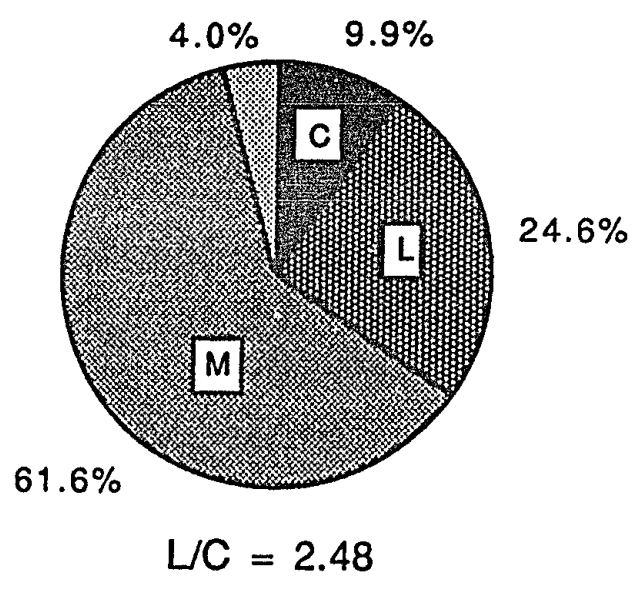


Fig. 8

\section{Percentages of retail land use on THE MAIN STREETS 1988}

HOLLISTER 1988 THE MAIN ST ONLY

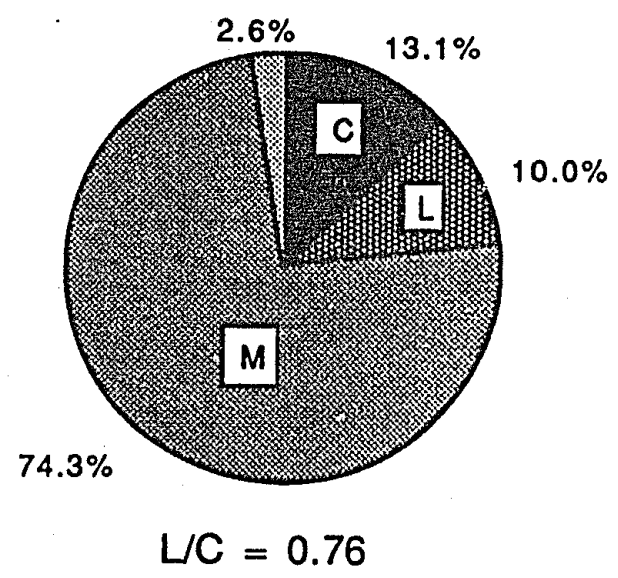

SANTA CRUZ 1988

THEMAIN STREET ONLY

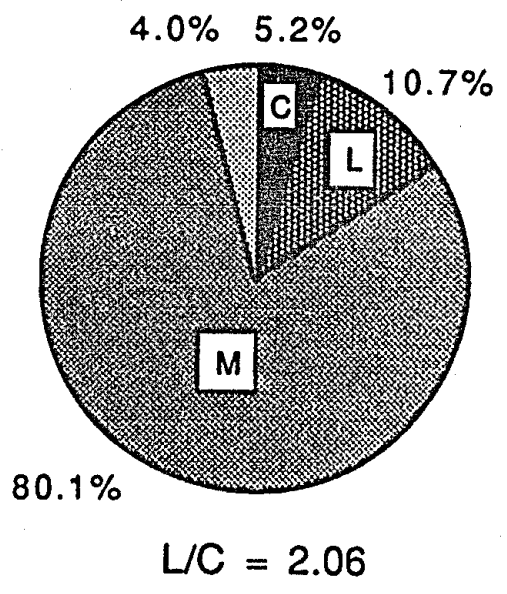

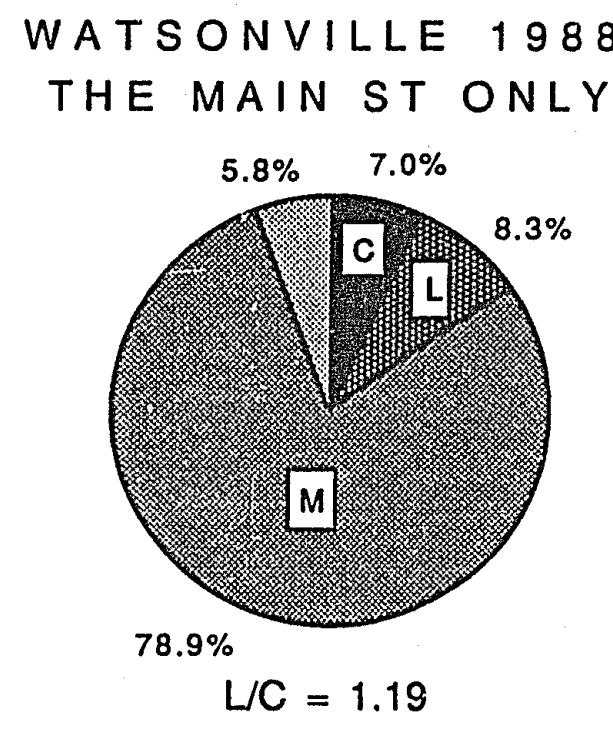

LOS GATOS 1988 THE MAIN STREET ONLY

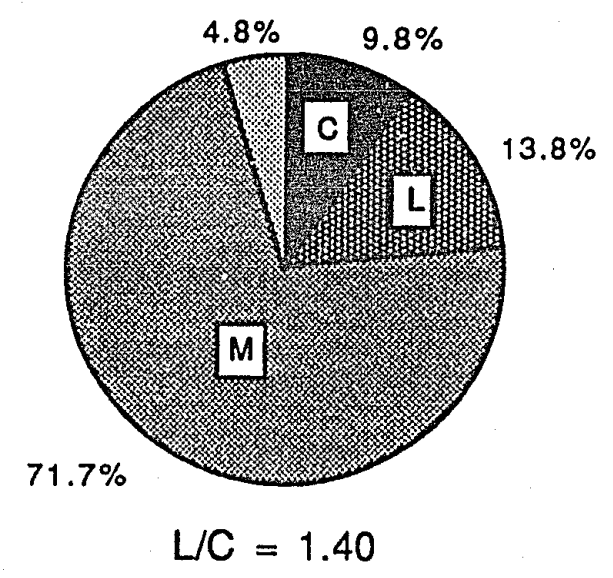


Santa Cruz is 2.46 , and 2.48 for Los Gatos. There are almost 2.5 times more limited appeal space than convenience space.

The same change is not as clear if one only looks at the percentage of limited appeal space in each CBD. In 1960, the division between the cities was distinct. Watsonville and Hollister have just under 4\% limited appeal space, while Santa Cruz and Los Gatos have just over $8 \%$. In 1988, as expected, Los Gatos is clearly the leader with $24.6 \%$ limited appeal space, almost doubling the percentage of limited appeal space in the other cities. By looking exclusively at the limited appeal percentage, however, Hollister would be second in the trend with limited appeal space at $13.8 \%$ of the total retail area. (see fig. 7) This relatively high percentage of limited appeal space, as stated above, largely reflects the lack of retail outside of the CBD in Hollister. Santa Cruz, conversely, shows a relative lack of limited appeal space because of the high percentage of retail space outside of the CBD. Likewise, if one looks at the smallness in the percentage of convenience space as an indicator of development, the results would also be deceptive. The ranking of the cities according to the smallness of the percentage of convenience space would be: Santa Cruz, Watsonville, Los Gatos, then Hollister. Los Gatos went from the highest percent of convenience space to the second highest percent. (see statistics tables)

In Santa Cruz and Los Gatos the movement of convenience stores to the periphery of the CBD from 1960 to 1988 can be distinctly seen. 
(see maps 13 and 16; 19 and 22) Many of the central blocks which had a significant number of convenience stores now have very few or no convenience establishments on them. Although this type of movement may be occurring in Hollister and Watsonville to some extent, it is not very noticeable. Convenience stores appear to be diminishing in number fairly evenly throughout the CBD. (see maps 1 and $4 ; 7$ and 10) Convenience stores still show a preference for corner locations. They are located on a much greater percentage of corner lots than their percentage of floorspace would indicate.

As people from the surrounding area travel to Santa Cruz and particularly to Los Gatos to shop at limited appeal stores, a clustering of these stores has appeared. Santa Cruz is beginning to show a clustering of limited appeal stores in the northern, "mall" area of Pacific Avenue. (see map 17) It is likely that limited appeal space will continue to grow in this part of the CBD. Los Gatos now has two distinct clusters of limited appeal stores: one on Main Street and one on Village. (see map 23) In Los Gatos these stores are predorninantly, although not exclusively, antique stores. (see table 31)

For all of the cities, convenience stores on average were smaller than either limited appeal stores of mass appeal stores. Their averages range from $109 \mathrm{~m}^{2}$ to $161 \mathrm{~m}^{2}$. Limited appeal stores range in average from $129 \mathrm{~m}^{2}$ to $214 \mathrm{~m}^{2}$. Mass appeal stores range is size from $201 \mathrm{~m}^{2}$ to $372 \mathrm{~m}^{2}$. Although these averages are significantly different, their standard deviations are so large that using store size 
would not be a good way to predict type. The average size store in Los Gatos was smaller than the average size store in the other cities. Because the cost per square meter of floorspace has increased in Los Gatos, stores must either become smaller, move to a lower rent area, or generate more revenue. Los Gatos is also the only city in the study with no large department store within the CBD. Hollister, Watsonville, and Santa Cruz, all have large department stores on the "main street." Because Los Gatos is close to other cities, the residents have numerous shopping centers close by to choose from. The residents of the other cities in the study, however, must drive long distances to find an alternative.

The increase in disposable income in all four cities has an impact on the amount of space that the city can support of individual types of stores. Part of the increase in financial institutions and restaurants can be explained by this market change. As people have a greater disposable income, they can afford to eat out more frequently. Santa Cruz and Los Gatos have had the highest percent increase in restaurant floorspace partially because of the increased income. Gas stations and corner drug stores have decreased or disappeared from the CBDs because they are unable to compete for space with higher profit generating establishments. Large chain stores, like "Longs," have taken away much of the market from convenience type drug stores. They are more profitable, carry a greater variety of goods at cheaper prices, and have become truly mass appeal retail 
establishments. They are also rarely located within the CBD, but are frequently found in small shopping centers, located at major intersections. One such store in Los Gatos is located in this type of shopping center just outside of the CBD on the southern corner of Saratoga Avenue and N. Santa Cruz Avenue. Grocery stores have also changed to large mass appeal stores located close to, but not usually in, the CBD. These market changes are true for all of the cities in the study.

The names of the clothing stores are indicative of the character of the downtown. The stores in Hollister have simple names. The department stores are part of well known middle class chains. For instance, Hollister has a JC Penny, a Ford's department store, a Family Bargains Center, Pants Place etc.. Watsonville also has similar stores: a JC Penny, a Ford's, Watsonville Jeans, GB Family Wear etc.. Although Santa Cruz has a JC Penny and a Ford's, it also has stores called Ambience, Angelica's, Revelation, etc. A Ford's or JC Penny would not fit the character of Los Gatos. It has Idamina's Fashions, Andienne's, LF Elegance, Sona's Boutique, etc. This difference was not so apparent in 1960.

The limited appeal space in Los Gatos and Santa Cruz has grown, not only in total floorspace, but also in the degree of limited appeal. In 1960, the limited stores in all four cities were mostly marginally limited appeal stores. There were music stores, craft stores, glass and lumber stores, pet stores, and camera shops. In Hollister and 
Watsonville, this description still fits with very few exceptions. In Los Gatos and Santa Cruz, limited appeal establishments have become much more limited. There are antique stores, fancy restaurants, boutiques, and art galleries. (see tables ) It is unlikely that either Los Gatos of Santa Cruz could support so many extremely limited appeal stores if they had to depend on only the patronage of the residents of the city. Of course, the less limited limited appeal stores also exist in Los Gatos and Santa Cruz.

Because Santa Cruz and Los Gatos are now relatively closer to the large metropolitan population, the retail floorspace in the CBD has grown considerably. The retail floorspace in Santa Cruz's CBD increased by $46 \%$ and the floorspace in Los Gatos's CBD increased by 84.\%. Hollister's CBD only increased by $8 \%$ and Watsonville's floorspace decreased by over $6 \%$. If more people shop in the CBD of a city, it can support a greater amount of retail area. Population change, of course, accounts for part of this growth, as it does for Hollister, but it cannot be the only factor. Although Los Gatos has had the highest percent population change, it is only about $25 \%$ greater than the population change in Hollister. (see table 1) 


\section{CHAPTER V}

\section{CONCLUSION}

This study has applied both a quantitative and spatial method for anaiyzing the development of retail space within the central business districts of medium sized cities. The statistical data, and the pie graphs which were generated from them, produced the needed information for a quantitative study. Numbers, however, have no spatial quality to them. For this reason it was necessary to generate maps of the central business districts for the different time periods. The categories into which the retail space was divided--convenience space, limited appeal space, and mass appeal space--are general enough to allow for this method to be universally employed in studying other CBDs, or even in a study of these CBDs sometime in the future.

As stated in the introduction, the changes in the retail area of the CBDs are the result of both market changes and changes in the relative location of the city to a large metropolitan market. The movement from convenience drug stores and grocery stores to larger, mass appeal stores is mostly a result of market changes as is the increase in banking space. The increase in the percentage of restaurant space is a result of both market and propinquity changes. All four of the cities show an increase in the percentage of restaurant space, but the change is much greater in Los Gatos and in Santa Cruz. The increase in the percentage of limited appeal space is also a result of both factors. The extremely limited appeal 
stores, however, can only survive because of their propinquity to the large metropolitan area. The population of the cities is not large enough to support such limited appeal stores.

The comparison of Los Gatos, Santa Cruz, Watsonville, and Hollister from 1960 to 1988 , has clearly shown the trend in the development of the retail in central business districts. In the beginning, the CBDs contained mostly mass appeal space, a considerable amount of convenience space, and very little limited appeal space. Over time, the percentage of convenience space decreased, and the percentage of limited appeal space increased, to approximately equal amounts of each. The CBDs of Watsonville and Hollister are presently at this stage. As development continues, convenience space continues to decrease in percentage, and limited appeal increases in percentage. Convenience stores move to the periphery of the CBD. Limited appeal stores begin to cluster. Limited appeal stores also become much more limited in appeal. This is the present stage of the CBDs in Los Gatos and Santa Cruz. In the future, one would expect the percentage of convenience space to stabilize at some small percentage. Limited appeal space should continue to grow and cluster. Limited appeal stores will probably become more and more specialized. Mass appeal space throughout the stages has remained fairly constant. One would expect this percentage to remain fairly stable or decrease slightly as the increase in limited appeal space continues to grow. Watsonville and Hollister will also continue to evolve in a similar way along this same trend. The speed of their development is largely 
dependent on the outward growth of the San Francisco Bay metropolis. As the Bay Area continues to grow, the population of Watsonville and Hollister should increase because the cities will be in commuting distance of the major employment center.

Many related studies can be done to help understand the retail development of central business districts. A study of shopping frequency and periodicity would be helpful. In an isolated city one would expect a much more even distribution of shoppers throughout the week than one would expect in cities like Los Gatos and Santa Cruz which attract largely weekend shoppers. A comparison of retail rents would also be useful in understanding the transformation of the downtown. A knowledge of the amount of sales and prices charged would also be useful. The information found in the present study should be helpful for continuing the analysis of the evolving central business district. 


\section{REFERENCES}

California Statistical Abstract 1960. Sacramento: Economic Development agency, 1962. pp. 54-55, 222-224.

California Statistical Abstract 1987. Sacramento: Department of Finance, 1987. p. 19.

Carter, Harold. The Study of Urban Geography. New York: John Wiley and Sons, 1976.

Hartshorn, Truman A. Interpreting the City: An Urban Geography. New York: John Wiley and Sons. 1980.

Horner, Edith R. (Ed.) California cities. Towns and Counties. Palo Alto: Hornwood Press 1987. pp. 166, 221, 361, 423.

Polk Directory for Hollister. El Monte, CA: R. L. Polk and Co. Publishers, 1961.

Polk Directory for Los Gatos. El Monte, CA: R. L. Polk and Co. Publishers, 1960.

Polk Directory for Santa Cruz. El Monte, CA: R. L. Polk and Co. Publishers, 1960.

Polk Directory for Watsonville. El Monte, CA: R. L. Polk and Co. Publishers, 1960.

Standard Land Use Coding Manual. Washington D.C.: U.S. Department of Transportation, 1965.

Vance, James E. Jr. This Scene of Man: The Role and Structure of the City in the Geography of Western Civilization. New York: Harpers College Press, 1977. 